\title{
Copper-Catalyzed Direct Propargylation of Polyfluoroarenes with Secondary Propargyl Phosphates
}

Yan-Bo Yu, Zhi-Ji Luo, and Xingang Zhang*

Key Laboratory of Organofluorine Chemistry, Shanghai Institute of Organic Chemistry, Chinese Academy of Sciences, 345 Lingling Road, Shanghai 200032, China

xgzhang@mail.sioc.ac.cn 


\section{Table of Contents}

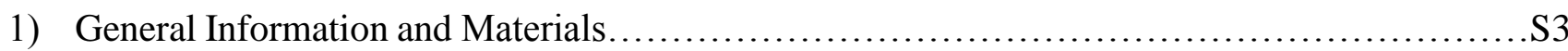

2) Screening for Copper-Catalyzed Cross-Coupling of Pentafluorobenzene 1a with Secondary

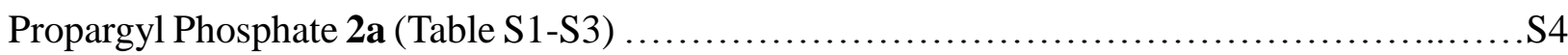

3) General Procedure for the Preparation of Secondary Propargyl Phosphates 2 .......................S6

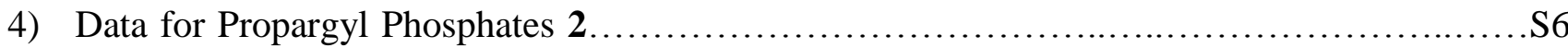

5) General Procedure for Cu-Catalyzed Cross-Coupling of Propargyl Phosphates 2 with

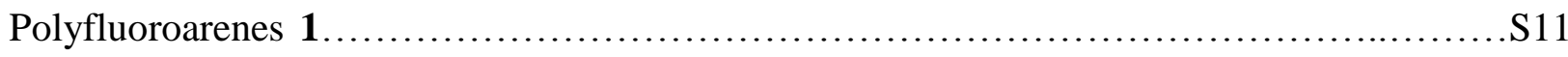

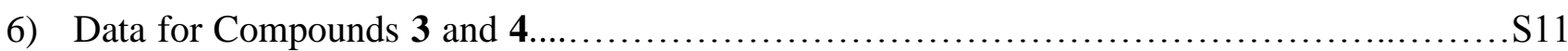

7) Unsymmetrical Synthesis of Dialkylated Fluoroarenes by Iterative C-H Functionalization of

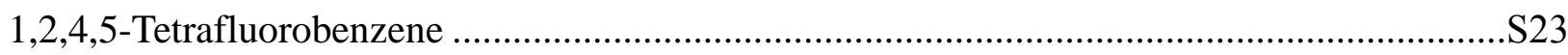

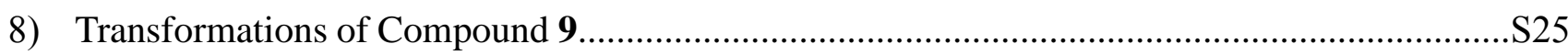

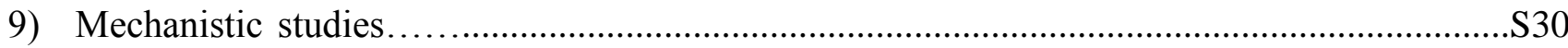

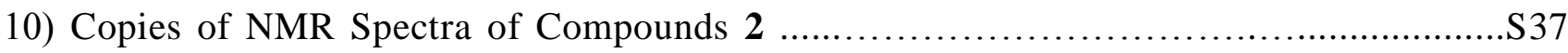

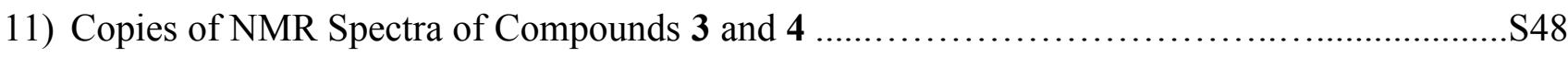

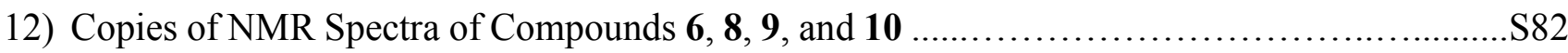

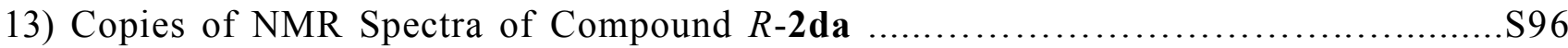

14) Copies of HPLC Analysis Spectra of Compounds $R$-2da, $R-2 d$, and $S$-3d...........................S98 
General information: ${ }^{1} \mathrm{H}$ NMR and ${ }^{13} \mathrm{C}$ NMR spectra were recorded on a Bruker AM400 and AM500 spectrometer. ${ }^{19} \mathrm{~F}$ NMR was recorded on a Bruker AM400 spectrometer $\left(\mathrm{CFCl}_{3}\right.$ as an external standard and low field is positive). Chemical shifts $(\delta)$ are reported in ppm, and coupling constants $(J)$ are in Hertz $(\mathrm{Hz})$. The following abbreviations were used to explain the multiplicities: $\mathrm{s}=$ singlet, $\mathrm{d}=\mathrm{doublet}$, $\mathrm{t}=$ triplet, $\mathrm{q}=$ quartet, $\mathrm{m}=$ multiplet, $\mathrm{br}=$ broad. $\mathrm{NMR}$ yield was determined by ${ }^{19} \mathrm{~F} \mathrm{NMR}$ using fluorobenzene as an internal standard before working up the reaction.

Materials: All reagents were used as received from commercial sources, unless specified otherwise. All reagents were weighed and handled in air, and refilled with an inert atmosphere of $\mathrm{N}_{2}$ at room temperature. THF was distilled from sodium and benzophenone before use. Propargyl phosphates were prepared from corresponding propargyl alcohol and diethyl chlorophosphate according to the literature. ${ }^{1}$ Dans- $\left(\mathrm{CH}_{2}\right)_{3}-\mathrm{N}_{3}$ was prepared according to the literature. ${ }^{2}$

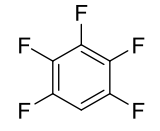

1a

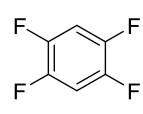

1b

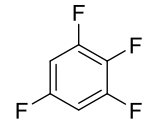

1c

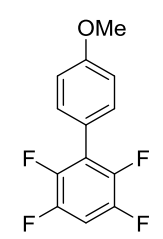

1d

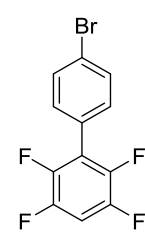

1e

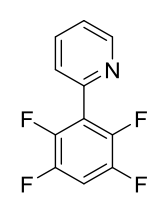

$1 \mathrm{f}$

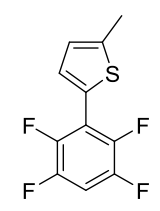

$1 \mathrm{~g}$

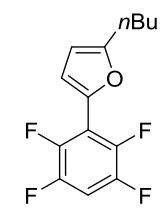

$1 \mathrm{~h}$

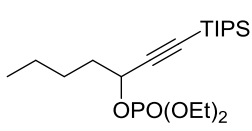

$2 a$

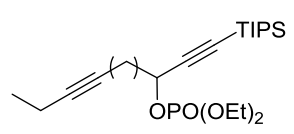

$2 f$

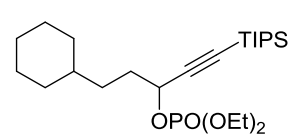

2b

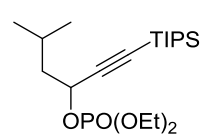

2c

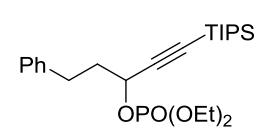

2d

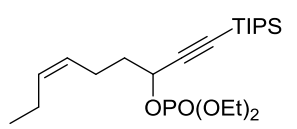

$2 \mathrm{e}$

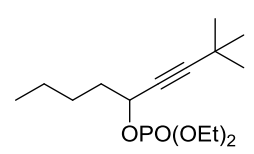

2i

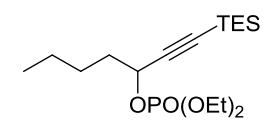

2j 2k

1 Uehling, M. R.; Marionni, S. T.; Lalic, G. Org. Lett. 2012, 14, 362.

${ }^{2}$ Frei, R.; Waser, J. J. Am. Chem. Soc. 2013, 135, 9620. 


\section{Screening for Copper-Catalyzed Cross-Coupling of Pentafluorobenzene 1a with Secondary}

Propargyl Phosphate 2a (Table S1-S3). To a septum capped $25 \mathrm{~mL}$ of Schlenck tube were added $[\mathrm{Cu}](10-20 \mathrm{~mol} \%)$, ligand (20 mol\%), base (1.2 - 2.4 equiv) under $\mathrm{N}_{2}$, followed by solvent ( $\left.1 \mathrm{~mL}\right)$. Propargyl phosphate 2a ( $0.3 \mathrm{mmol}, 1.0$ equiv) and pentafluorobenzene 1a (2.0 - 3.0 equiv) were then added. The tube was screw capped and put into an oil bath (preheated to 50 or $80{ }^{\circ} \mathrm{C}$ ). After stirring for $8 \mathrm{~h}$, the reaction mixture was cooled to room temperature. The yield was determined by ${ }^{19} \mathrm{~F}$ NMR before working up. If necessary, the reaction mixture was diluted with ethyl acetate, washed with brine, dried over $\mathrm{Na}_{2} \mathrm{SO}_{4}$, filtered and concentrated. The residue was purified with silica gel chromatography to provide pure product.

Table S1. Ligand Effect on Cu-Catalyzed Cross-Coupling of 1a with $2 a^{a}{ }^{a}$

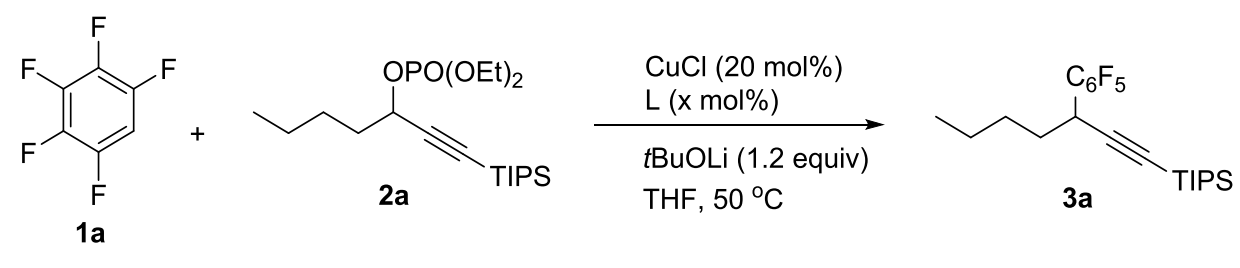

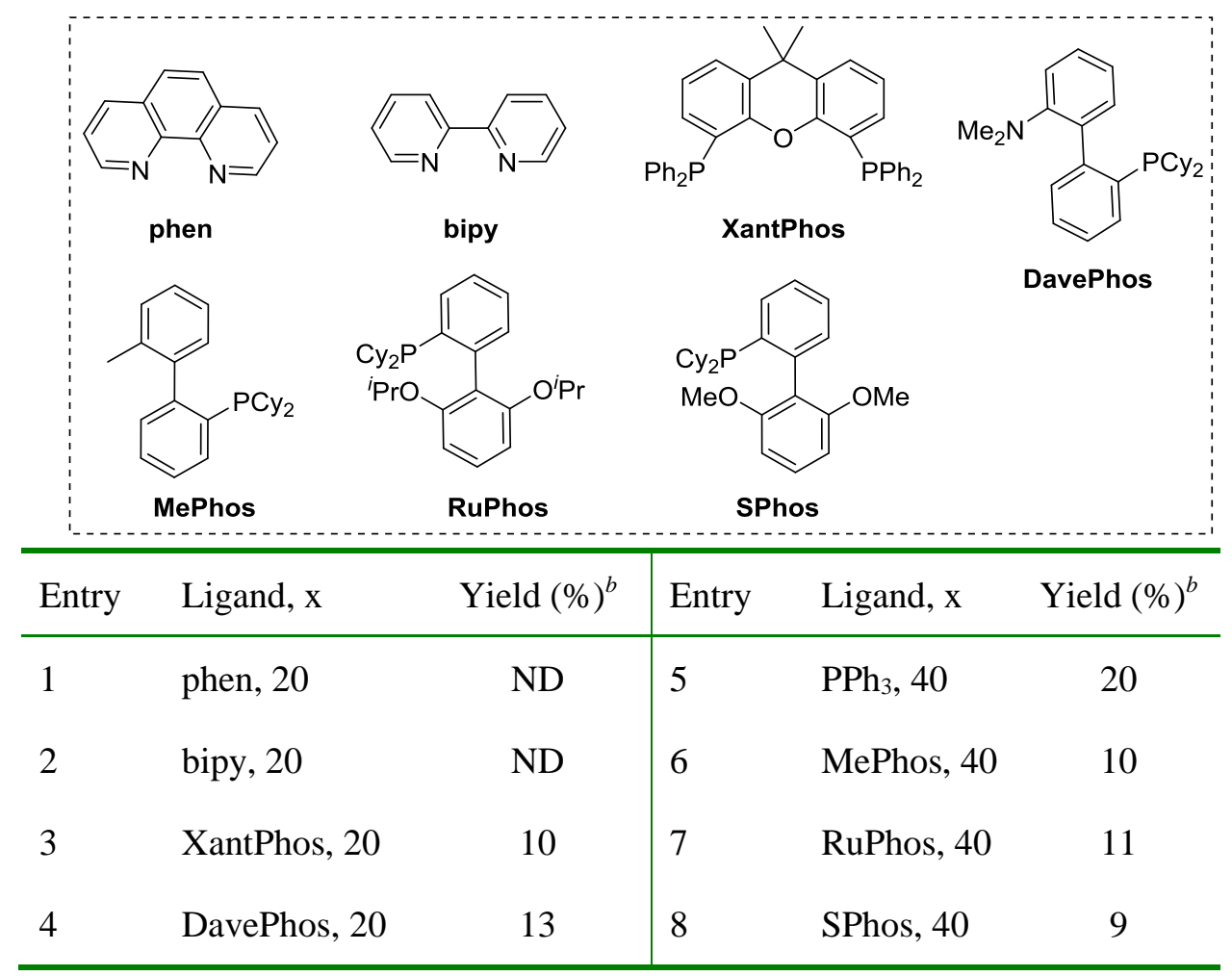

${ }^{a}$ Reaction conditions (unless otherwise specified): $1 \mathbf{a}(0.6 \mathrm{mmol}, 2.0$ equiv), $2 \mathbf{a}(0.3 \mathrm{mmol}, 1.0$ equiv), THF (1 mL), $50{ }^{\circ} \mathrm{C}, 8$ h. ${ }^{b}$ Determined by ${ }^{19} \mathrm{~F}$ NMR using fluorobenzene as an internal standard. ND = Not detected. 
Table S2. Screening of Copper Catalysts for Cross-Coupling of 1a with 2a. ${ }^{a}$
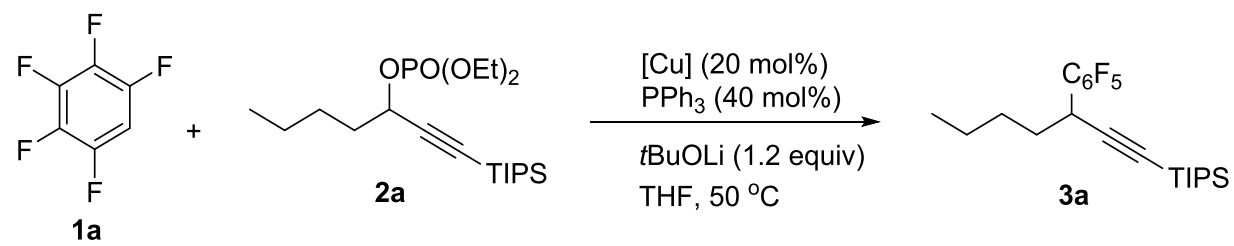

\begin{tabular}{cllc|clcc}
\hline Entry & {$[\mathrm{Cu}]$} & Ligand, $x$ & Yield $(\%)^{b}$ & Entry & {$[\mathrm{Cu}]$} & Ligand, $^{2}$ & ${\text { Yield }(\%)^{b}}^{b}$ \\
\hline 1 & $\mathrm{CuI}$ & $\mathrm{PPh}_{3}, 40$ & 21 & 5 & $\mathrm{Cu}(\mathrm{OAc})_{2}$ & $\mathrm{PPh}_{3}, 40$ & 15 \\
2 & $\mathrm{CuBr}$ & $\mathrm{PPh}_{3}, 40$ & 18 & 6 & $\mathrm{CuOAc}$ & $\mathrm{PPh}_{3}, 40$ & 30 \\
3 & $\mathrm{CuCl}_{2}$ & $\mathrm{PPh}_{3}, 40$ & $\mathrm{ND}$ & 7 & $\mathrm{CuOAc}$ & $/$ & 45 \\
4 & $\mathrm{CuSCN}$ & $\mathrm{PPh}_{3}, 40$ & 23 & $8^{c}$ & $\mathrm{CuOAc}$ & $/$ & 68 \\
\hline
\end{tabular}

${ }^{a}$ Reaction conditions (unless otherwise specified): 1a $(0.6 \mathrm{mmol}, 2.0$ equiv), $\mathbf{2 a}(0.3 \mathrm{mmol}, 1.0$ equiv), THF ( $1 \mathrm{~mL})$, $50{ }^{\circ} \mathrm{C}, 8 \mathrm{~h} .{ }^{b}$ Determined by ${ }^{19} \mathrm{~F}$ NMR using fluorobenzene as an internal standard. ${ }^{c}$ Reaction run at $80{ }^{\circ} \mathrm{C}$.

Table S3. Screening of Solvents and Bases for Cu-Catalyzed Cross-Coupling of 1a with 2a. ${ }^{a}$<smiles>Fc1cc(F)c(F)c(F)c1F</smiles>

1a<smiles>CCCCC(C#C[In]S)O[R]OCC</smiles>

2a
CuOAc (20 mol\%)

Base (x equiv)

Solvent, $80^{\circ} \mathrm{C}$

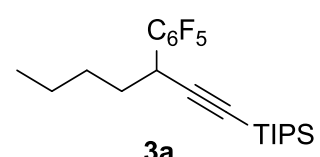

$3 a$

\begin{tabular}{cccc|cccc}
\hline Entry & Base, $\mathrm{x}$ & Solvent & Yield $(\%)^{b}$ & Entry & Base, $\mathrm{x}$ & Solvent & Yield $(\%)^{b}$ \\
\hline 1 & $t$ BuOLi, 1.2 & Toluene & 20 & 5 & $t \mathrm{BuOK}, 1.2$ & THF & 40 \\
2 & $t$ BuOLi, 1.2 & Dioxane & $\mathrm{ND}$ & 6 & $\mathrm{~K}_{2} \mathrm{CO}_{3}, 1.2$ & THF & ND \\
3 & $t$ BuOLi, 1.2 & DMF & $\mathrm{ND}$ & 7 & $t \mathrm{BuOLi}, 2.4$ & THF & 75 \\
4 & $t$ BuONa, 1.2 & THF & 55 & $8^{c}$ & $t$ BuOLi, 2.4 & THF & $90(85)$ \\
\hline
\end{tabular}

${ }^{a}$ Reaction conditions (unless otherwise specified): $1 \mathbf{a}(0.6 \mathrm{mmol}, 2.0$ equiv), $\mathbf{2 a}(0.3 \mathrm{mmol}, 1.0$ equiv),THF $(1 \mathrm{~mL})$, $80{ }^{\circ} \mathrm{C}, 8 \mathrm{~h} .{ }^{b}$ Determined by ${ }^{19} \mathrm{~F}$ NMR using fluorobenzene as an internal standard. ${ }^{c} \mathbf{1 a}$ (1.8 mmol, 3.0 equiv), 2a (0.6 mmol, 1.0 equiv), CuOAc (10 mol\%), THF $(2 \mathrm{~mL}), 80^{\circ} \mathrm{C}, 12 \mathrm{~h}$. Number in parenthesis is isolated yield. 


\section{General Procedure for the Preparation of Secondary Propargyl Phosphates 2}

To a solution of (triisopropylsilyl)acetylene (1.0 equiv) in THF was added dropwise $n$ BuLi (1.6 M hexane solution, 1.0 equiv) at $0{ }^{\circ} \mathrm{C}$ under $\mathrm{N}_{2}$. After the resulting mixture was stirred for 30 min at same temperature, the corresponding aldehyde (1.0 equiv) was added via a syringe. The resulting mixture was allowed to warm to room temperature and stirred for additional $2 \mathrm{~h}$. The resulting suspension was quenched with saturated aq. $\mathrm{NH}_{4} \mathrm{Cl}$ and extracted with ethyl acetate three times. The combined organic layers were dried over sodium sulfate and concentrated. The residue was used directly in the next step without further purification.

To a flask were added propargylic alcohol (1.0 equiv), 4-dimethylaminopyridine (0.1 equiv), dry $\mathrm{CH}_{2} \mathrm{Cl}_{2}$, and diethylchlorophosphate (1.3 equiv) under $\mathrm{N}_{2}$. The resulting mixture was then cooled to $0{ }^{\circ} \mathrm{C}$ and triethylamine (1.2 equiv) was added. The reaction mixture was allowed to warm to room temperature with stirring. After consumption of the alcohol (monitored by TLC), the mixture was quenched with saturated aq. $\mathrm{NH}_{4} \mathrm{Cl}$ and extracted with ethyl acetate three times. The organic phase was washed with brine, dried over $\mathrm{Na}_{2} \mathrm{SO}_{4}$, and concentrated. The residue was purified by silica gel chromatography to give desired propargyl phosphates.

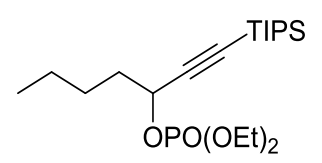

Diethyl (1-(triisopropylsilyl)hept-1-yn-3-yl) phosphate (2a). The product (73\% overall yield, 2 steps) as a colorless oil was purified with silica gel chromatography (Pentane / EtOAc 4:1). ${ }^{1} \mathrm{H}$ NMR (400 $\left.\mathrm{MHz}, \mathrm{CDCl}_{3}\right) \delta 5.00(\mathrm{dd}, J=14.2,6.7 \mathrm{~Hz}, 1 \mathrm{H}), 4.17-4.07(\mathrm{~m}, 4 \mathrm{H}), 1.85-1.78(\mathrm{~m}, 2 \mathrm{H}), 1.50-1.43(\mathrm{~m}$, $2 \mathrm{H}), 1.38-1.30(\mathrm{~m}, 8 \mathrm{H}), 1.05-0.94(\mathrm{~m}, 21 \mathrm{H}), 0.89(\mathrm{t}, J=7.2 \mathrm{~Hz}, 3 \mathrm{H}) .{ }^{13} \mathrm{C} \mathrm{NMR}\left(126 \mathrm{MHz}, \mathrm{CDCl}_{3}\right) \delta$ $104.6(\mathrm{~d}, J=4.3 \mathrm{~Hz}), 87.6,68.5(\mathrm{~d}, J=5.7 \mathrm{~Hz}), 63.7(\mathrm{dd}, J=5.7 \mathrm{~Hz}, J=4.2 \mathrm{~Hz}), 36.4(\mathrm{~d}, J=5.7 \mathrm{~Hz})$, 26.8, 22.0, 18.4, $16.0(\mathrm{dd}, J=7.0,3.3 \mathrm{~Hz}), 13.8,11.0$. IR (thin film) $v_{\max } 2944,2867,2176,1724,1034$ $\mathrm{cm}^{-1}$. MS (EI): $\mathrm{m} / z(\%) 405[\mathrm{M}+\mathrm{H}]^{+}$. HRMS: Calculated for $\mathrm{C}_{20} \mathrm{H}_{42} \mathrm{O}_{4} \mathrm{PSi}$ : 405.2584; Found: 405.2582 . 


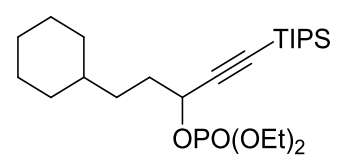

5-Cyclohexyl-1-(triisopropylsilyl)pent-1-yn-3-yl diethyl phosphate (2b). The product (65\% overall yield, 2 steps) as a colorless oil was purified with silica gel chromatography (Pentane / EtOAc 3:1). ${ }^{1} \mathrm{H}$ NMR $\left(500 \mathrm{MHz}, \mathrm{CDCl}_{3}\right) \delta 4.81(\mathrm{dd}, J=14.0,6.7 \mathrm{~Hz}, 1 \mathrm{H}), 3.97-3.89(\mathrm{~m}, 4 \mathrm{H}), 1.66-1.63(\mathrm{~m}, 2 \mathrm{H})$, 1.51-1.44 (m, 6H), 1.23-1.19 (m, 2H), 1.16-1.13 (m, 5H), 1.17-0.93 (m, 4H), 0.95-0.79 (m, 21H), 0.74$0.67(\mathrm{~m}, 2 \mathrm{H}) .{ }^{13} \mathrm{C} \mathrm{NMR}\left(126 \mathrm{MHz}, \mathrm{CDCl}_{3}\right) \delta 104.6(\mathrm{~d}, J=4.4 \mathrm{~Hz}), 87.3,77.4,68.5(\mathrm{~d}, J=5.6 \mathrm{~Hz})$, $63.4(\mathrm{dd}, J=5.7,3.7 \mathrm{~Hz}), 36.9,34.1(\mathrm{~d}, J=5.6 \mathrm{~Hz}), 33.1,33.0,32.1,26.4,26.0,18.3,15.8(\mathrm{dd}, J=$ 6.9, $4.8 \mathrm{~Hz}$ ), 10.9. IR (thin film) $v_{\max } 2925,2865,2176,1035,679 \mathrm{~cm}^{-1}$. MS (DART): $\mathrm{m} / z$ (\%) 459 $[\mathrm{M}+\mathrm{H}]^{+}$. HRMS: Calculated for $\mathrm{C}_{24} \mathrm{H}_{48} \mathrm{O}_{4} \mathrm{PSi}$ : 459.3054; Found: 459.3052 .

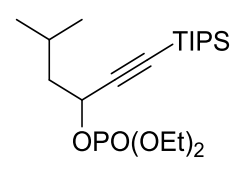

Diethyl (5-methyl-1-(triisopropylsilyl)hex-1-yn-3-yl) phosphate (2c). The product (70\% overall yield, 2 steps) as a colorless oil was purified with silica gel chromatography (Pentane / EtOAc 4:1). ${ }^{1} \mathrm{H}$ NMR $\left(400 \mathrm{MHz}, \mathrm{CDCl}_{3}\right) \delta 4.90(\mathrm{q}, J=7.4 \mathrm{~Hz}, 1 \mathrm{H}), 4.03-3.94(\mathrm{~m}, 4 \mathrm{H}), 1.79-1.71(\mathrm{~m}, 1 \mathrm{H}), 1.66-$ $1.60(\mathrm{~m}, 1 \mathrm{H}), 1.55-1.49(\mathrm{~m}, 1 \mathrm{H}), 1.18(\mathrm{dd}, J=7.1,0.7 \mathrm{~Hz}, 6 \mathrm{H}), 0.98-0.86(\mathrm{~m}, 21 \mathrm{H}), 0.83(\mathrm{~d}, J=6.6$ $\mathrm{Hz}, 3 \mathrm{H}), 0.79(\mathrm{~d}, J=6.7 \mathrm{~Hz}, 3 \mathrm{H}) .{ }^{13} \mathrm{C} \mathrm{NMR}\left(126 \mathrm{MHz}, \mathrm{CDCl}_{3}\right) \delta 104.7(\mathrm{~d}, J=3.7 \mathrm{~Hz}), 87.5,67.1(\mathrm{~d}$, $J=5.8 \mathrm{~Hz}), 63.5(\mathrm{dd}, J=5.9,2.9 \mathrm{~Hz}), 45.6(\mathrm{~d}, J=6.0 \mathrm{~Hz}), 24.5,22.2,22.1,18.3,15.9(\mathrm{dd}, J=7.0$, $4.5 \mathrm{~Hz}), 10.9$. IR (thin film) $v_{\max } 2958,2867,2176,1279,679 \mathrm{~cm}^{-1}$. MS (EI): $m / z(\%) 361\left(\mathrm{M}_{-} \mathrm{C}_{3} \mathrm{H}_{7}\right)^{+}$, 267, 211 (100). HRMS: Calculated for $\mathrm{C}_{17} \mathrm{H}_{34} \mathrm{O}_{4} \mathrm{PSi}$ : 361.1964; Found: 361.1956.

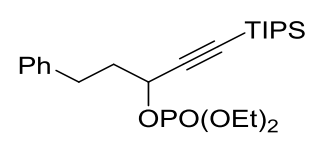

Diethyl (5-phenyl-1-(triisopropylsilyl)pent-1-yn-3-yl) phosphate (2d). The product (79\% overall yield, 2 steps) as a colorless oil was purified with silica gel chromatography (Pentane / EtOAc 3:1). ${ }^{1} \mathrm{H}$ NMR (400 MHz, $\left.\mathrm{CDCl}_{3}\right) \delta$ 7.31-7.26 (m, 2H), 7.21 - $7.20(\mathrm{~m}, 3 \mathrm{H}), 5.06(\mathrm{dd}, J=13.8,6.5 \mathrm{~Hz}, 1 \mathrm{H})$, 4.17-4.11 (m, 4H), $2.84(\mathrm{t}, J=8.0 \mathrm{~Hz}, 2 \mathrm{H}), 2.18-2.11(\mathrm{~m}, 2 \mathrm{H}), 1.34(\mathrm{td}, J=7.0,2.1 \mathrm{~Hz}, 6 \mathrm{H}), 1.08-1.07$ 
(m, 21H). ${ }^{13} \mathrm{C}$ NMR $\left(126 \mathrm{MHz}, \mathrm{CDCl}_{3}\right) \delta 140.8,128.5,128.4,126.1,104.2(\mathrm{~d}, J=4.3 \mathrm{~Hz}), 88.3,68,0$ $(\mathrm{d}, J=5.7 \mathrm{~Hz}), 63.8(\mathrm{dd}, J=5.9,2.7 \mathrm{~Hz}), 38.7(\mathrm{~d}, J=5.8 \mathrm{~Hz}), 31.1,18.5,16.1(\mathrm{dd}, J=6.8,5.6 \mathrm{~Hz})$,

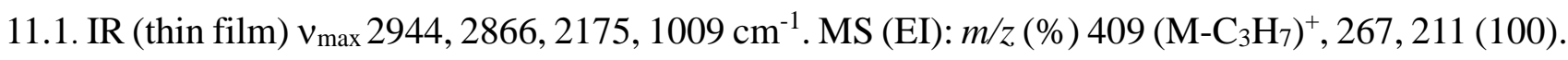
HRMS: Calculated for $\mathrm{C}_{21} \mathrm{H}_{34} \mathrm{O}_{4} \mathrm{PSi}$ : 409.1964; Found: 409.1972.

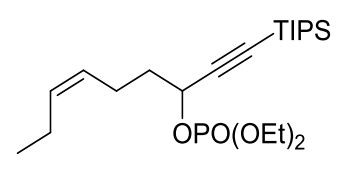

(Z)-Diethyl (1-(triisopropylsilyl)non-6-en-1-yn-3-yl) phosphate (2e). The product (63\% overall yield, 2 steps) as a colorless oil was purified with silica gel chromatography (Pentane / EtOAc 3:1). ${ }^{1} \mathrm{H}$ NMR $\left(400 \mathrm{MHz}, \mathrm{CDCl}_{3}\right) \delta 5.39(\mathrm{dt}, J=10.5,7.1 \mathrm{~Hz}, 1 \mathrm{H}), 5.30(\mathrm{dt}, J=8.6,7.2 \mathrm{~Hz}, 1 \mathrm{H}), 5.00(\mathrm{dd}$, $J=14.2,6.6 \mathrm{~Hz}, 1 \mathrm{H}), 4.16-4.07(\mathrm{~m}, 4 \mathrm{H}), 2.23-2.19(\mathrm{~m}, 2 \mathrm{H}), 2.06-1.99(\mathrm{~m}, 2 \mathrm{H}), 1.87-1.81(\mathrm{~m}, 2 \mathrm{H})$, $1.31(\mathrm{t}, J=7.0 \mathrm{~Hz}, 6 \mathrm{H}), 1.08-1.05(\mathrm{~m}, 21 \mathrm{H}), 0.92(\mathrm{t}, J=7.5 \mathrm{~Hz}, 3 \mathrm{H}) .{ }^{13} \mathrm{C} \mathrm{NMR}\left(126 \mathrm{MHz}, \mathrm{CDCl}_{3}\right) \delta$ 132.9, 126.9, $104.3(\mathrm{~d}, J=4.4 \mathrm{~Hz}), 87.9,68.1(\mathrm{~d}, J=5.7 \mathrm{~Hz}), 67.0,63.7$ (d, $J=5.9 \mathrm{~Hz}, 5.7 \mathrm{~Hz}), 36.9$ $(\mathrm{d}, J=5.6 \mathrm{~Hz}), 22.5,20.4,18.4,16.0(\mathrm{~d}, J=6.9 \mathrm{~Hz}, 7.0 \mathrm{~Hz}), 14.2,11.0$. IR (thin film) $v_{\max } 2944,2867$,

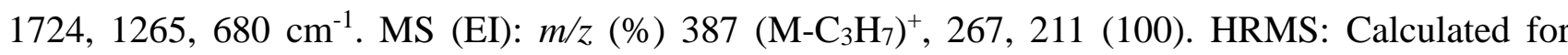
$\mathrm{C}_{19} \mathrm{H}_{36} \mathrm{O}_{4} \mathrm{SiP}: 387.2121$; Found: 387.2126.

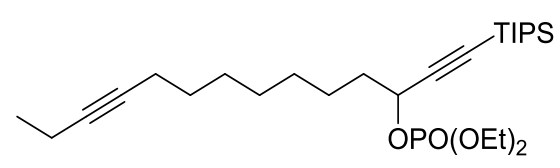

Diethyl (1-(triisopropylsilyl)tetradeca-1,11-diyn-3-yl) phosphate (2f). The product (68\% overall yield, 2 steps) as a colorless oil was purified with silica gel chromatography (Pentane / EtOAc 2:1). ${ }^{1} \mathrm{H}$ NMR $\left(400 \mathrm{MHz}, \mathrm{CDCl}_{3}\right) \delta 4.99(\mathrm{dd}, J=13.9,6.8 \mathrm{~Hz}, 1 \mathrm{H}), 4.14-4.07$ (m, 4H), 2.14-2.08 (m, 4H), 1.85-1.73 (m, 2H), 1.47-1.41 (m, 4H), 1.33-1.29 (m, 12H), 1.11-1.04 (m, 24H). ${ }^{13} \mathrm{C}$ NMR (126 MHz, $\left.\mathrm{CDCl}_{3}\right) \delta 104.6(\mathrm{~d}, J=4.4 \mathrm{~Hz}), 87.6,81.4,79.3,68.4(\mathrm{~d}, J=5.7 \mathrm{~Hz}), 63.6(\mathrm{dd}, J=5.8,3.9 \mathrm{~Hz}), 36.7$ $(\mathrm{d}, J=5.6 \mathrm{~Hz}), 29.0,28.9,28.8,28.6,24.6,18.6,18.4,16.0(\mathrm{dd}, J=7.0,4.0 \mathrm{~Hz}), 14.3,12.3,11.0 . \mathrm{IR}$ (thin film) $v_{\max } 3397,2938,2865,2175,1265,1035 \mathrm{~cm}^{-1}$. MS (DART): $m / z(\%) 499[\mathrm{M}+\mathrm{H}]^{+}$. HRMS: Calculated for $\mathrm{C}_{27} \mathrm{H}_{52} \mathrm{O}_{4} \mathrm{PSi}$ : 499.3367; Found: 499.3367 . 


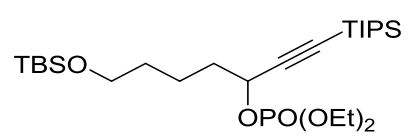

7-((tert-Butyldimethylsilyl)oxy)-1-(triisopropylsilyl)hept-1-yn-3-yl diethyl phosphate (2g). The product (61\% overall yield, 2 steps) as a colorless oil was purified with silica gel chromatography (Pentane / EtOAc 2:1). ${ }^{1} \mathrm{H}$ NMR (500 MHz, $\left.\mathrm{CDCl}_{3}\right) \delta 4.96(\mathrm{dd}, J=14.3,6.5 \mathrm{~Hz}, 1 \mathrm{H}), 4.12$ - 4.02 (m, 4H), 3.56 - $3.54(\mathrm{~m}, 2 \mathrm{H}), 1.83-1.78(\mathrm{~m}, 2 \mathrm{H}), 1.54-1.49(\mathrm{~m}, 4 \mathrm{H}), 1.29-1.26(\mathrm{~m}, 6 \mathrm{H}), 1.02$ - $0.95(\mathrm{~m}$, $21 \mathrm{H}), 0.83(\mathrm{~s}, 9 \mathrm{H}),-0.02(\mathrm{~s}, 6 \mathrm{H}) .{ }^{13} \mathrm{C} \mathrm{NMR}\left(126 \mathrm{MHz}, \mathrm{CDCl}_{3}\right) \delta 104.5(\mathrm{~d}, J=4.3 \mathrm{~Hz}), 87.7,68.4(\mathrm{~d}$, $J=5.7 \mathrm{~Hz}), 63.7(\mathrm{dd}, J=5.6,4.9 \mathrm{~Hz}), 62.9,36.7(\mathrm{~d}, J=5.7 \mathrm{~Hz}), 32.2,25.9,21.3,18.4,18.2,16.0(\mathrm{dd}$, $J=7.0,4.0 \mathrm{~Hz}$ ), 11.0, -5.4. IR (thin film) $v_{\max } 3435,2944,2866,2176,1036 \mathrm{~cm}^{-1}$. MS (DART): $\mathrm{m} / \mathrm{z}$ (\%) $535[\mathrm{M}+\mathrm{H}]^{+}$. HRMS: Calculated for $\mathrm{C}_{26} \mathrm{H}_{56} \mathrm{O}_{5} \mathrm{PSi}_{2}$ : 535.3398; Found: 535.3398.

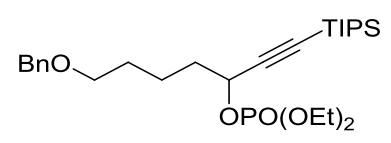

7-(Benzyloxy)-1-(triisopropylsilyl)hept-1-yn-3-yl diethyl phosphate (2h). The product (57\% overall yield, 2 steps) as a colorless oil was purified with silica gel chromatography (Pentane / EtOAc 2:1). ${ }^{1} \mathrm{H}$ NMR $\left(500 \mathrm{MHz}, \mathrm{CDCl}_{3}\right) \delta$ 7.33-7.31 (m, 4H), $7.29-7.25(\mathrm{~m}, 1 \mathrm{H}), 5.03(\mathrm{dd}, J=14.2,6.5 \mathrm{~Hz}$, 1H), 4.49 (d, $J=3.2 \mathrm{~Hz}, 2 \mathrm{H}), 4.17-4.08(\mathrm{~m}, 4 \mathrm{H}), 3.47(\mathrm{t}, J=6.0 \mathrm{~Hz}, 2 \mathrm{H}), 1.89-1.83(\mathrm{~m}, 2 \mathrm{H}), 1.69$ $1.60(\mathrm{~m}, 4 \mathrm{H}), 1.34-1.30(\mathrm{~m}, 6 \mathrm{H}), 1.06-1.03(\mathrm{~m}, 21 \mathrm{H}) .{ }^{13} \mathrm{C} \mathrm{NMR}\left(126 \mathrm{MHz}, \mathrm{CDCl}_{3}\right) \delta 138.5,128.3$, 127.5, 127.4, $104.5(\mathrm{~d}, J=4.2 \mathrm{~Hz}), 87.8,72.8,70.0,68.3(\mathrm{~d}, J=5.7 \mathrm{~Hz}), 63.7(\mathrm{dd}, J=5.6,3.0 \mathrm{~Hz})$, $36.6(\mathrm{~d}, J=5.7 \mathrm{~Hz}), 29.1,21.6,18.5,16.0(\mathrm{dd}, J=7.0,2.2 \mathrm{~Hz}), 11.0$. IR (thin film) $v_{\max } 3327,2943$, 2866, 2176, 1719, $1034 \mathrm{~cm}^{-1}$. MS (DART): $\mathrm{m} / \mathrm{z}(\%) 511[\mathrm{M}+\mathrm{H}]^{+}, 490$. HRMS: Calculated for $\mathrm{C}_{27} \mathrm{H}_{48} \mathrm{O}_{5} \mathrm{PSi}$ : 511.3003; Found: 511.3003.

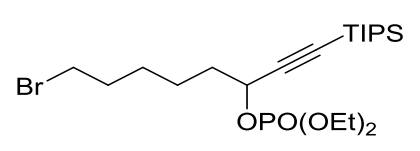

8-Bromo-1-(triisopropylsilyl)oct-1-yn-3-yl diethyl phosphate (2i). The product (59\% overall yield, 2 steps) as a colorless oil was purified with silica gel chromatography (Pentane / EtOAc 3:1). ${ }^{1} \mathrm{H}$ NMR $\left(500 \mathrm{MHz}, \mathrm{CDCl}_{3}\right) \delta 5.00(\mathrm{dd}, J=13.7,6.8 \mathrm{~Hz}, 1 \mathrm{H}), 4.15-4.09(\mathrm{~m}, 4 \mathrm{H}), 3.38(\mathrm{t}, J=6.7 \mathrm{~Hz}, 2 \mathrm{H}), 1.87-$ 
$1.79(\mathrm{~m}, 4 \mathrm{H}), 1.55-1.46(\mathrm{~m}, 4 \mathrm{H}), 1.32(\mathrm{t}, J=7.0 \mathrm{~Hz}, 6 \mathrm{H}), 1.05-1.03(\mathrm{~m}, 21 \mathrm{H}) .{ }^{13} \mathrm{C} \mathrm{NMR}(126 \mathrm{MHz}$, $\left.\mathrm{CDCl}_{3}\right) \delta 104.3,87.9,68.2(\mathrm{~d}, J=5.5 \mathrm{~Hz}), 63.7(\mathrm{dd}, J=4.7,3.5 \mathrm{~Hz}), 36.5(\mathrm{~d}, J=5.5 \mathrm{~Hz}), 33.3,32.5$, 27.5, 23.8, 18.4, $16.0(\mathrm{dd}, J=6.5,4.8 \mathrm{~Hz}), 11.0$. IR (thin film) $v_{\max } 2943,2866,2176,1724,1006 \mathrm{~cm}^{-}$ 1. MS (DART): $m / z(\%) 497[\mathrm{M}+\mathrm{H}]^{+}$. HRMS: Calculated for $\mathrm{C}_{21} \mathrm{H}_{42} \mathrm{BrO}_{4} \mathrm{PSi}$ : 497.1846; Found: 497.1846.

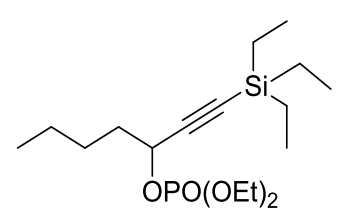

Diethyl (1-(triethylsilyl)hept-1-yn-3-yl) phosphate (2j). The product (70\% overall yield, 2 steps) as a colorless oil was purified with silica gel chromatography (Pentane / EtOAc 4:1). ${ }^{1} \mathrm{H}$ NMR (500 MHz, $\left.\mathrm{CDCl}_{3}\right) \delta 4.97(\mathrm{dd}, J=13.7,6.8 \mathrm{~Hz}, 1 \mathrm{H}), 4.14-4.09(\mathrm{~m}, 4 \mathrm{H}), 1.83-1.76(\mathrm{~m}, 2 \mathrm{H}), 1.48-1.36(\mathrm{~m}, 2 \mathrm{H})$, $1.34-1.30(\mathrm{~m}, 8 \mathrm{H}), 0.96(\mathrm{t}, J=7.9 \mathrm{~Hz}, 9 \mathrm{H}), 0.89(\mathrm{t}, J=7.3 \mathrm{~Hz}, 3 \mathrm{H}), 0.57(\mathrm{q}, J=7.9 \mathrm{~Hz}, 6 \mathrm{H}) .{ }^{13} \mathrm{C} \mathrm{NMR}$ $\left(126 \mathrm{MHz} \mathrm{CDCl}_{3}\right) \delta 103.7,88.5,68.3,63.6,36.1,26.7,21.9,15.8,13.6,7.0,4.0$. IR (thin film) $v_{\max }$ 2957, 2876, 2177, 1725, $1264 \mathrm{~cm}^{-1}$. MS (EI): $\mathrm{m} / z$ (\%) $347\left(\mathrm{M}-\mathrm{CH}_{3}\right)^{+}, 239,183$ (100). HRMS: Calculated for $\mathrm{C}_{15} \mathrm{H}_{30} \mathrm{O}_{4} \mathrm{SiP}: 333.1651$; Found: 333.1649 .

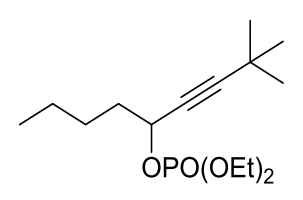

2,2-Dimethylnon-3-yn-5-yl diethyl phosphate (2k). The product (62\% overall yield, 2 steps) as a colorless oil was purified with silica gel chromatography (Pentane / EtOAc 5:1). ${ }^{1} \mathrm{H}$ NMR (400 MHz, $\left.\mathrm{CDCl}_{3}\right) \delta 4.88(\mathrm{q}, J=6.8 \mathrm{~Hz}, 1 \mathrm{H}), 4.10-4.00(\mathrm{~m}, 4 \mathrm{H}), 1.75-1.64(\mathrm{~m}, 2 \mathrm{H}), 1.38-1.24(\mathrm{~m}, 10 \mathrm{H}), 1.13(\mathrm{~s}$, $9 \mathrm{H}), 0.83(\mathrm{t}, J=7.2 \mathrm{~Hz}, 3 \mathrm{H}) .{ }^{13} \mathrm{C} \mathrm{NMR}\left(126 \mathrm{MHz}, \mathrm{CDCl}_{3}\right) \delta 95.2,76.2(\mathrm{~d}, J=4.1 \mathrm{~Hz}), 68.5(\mathrm{~d}, J=$ $5.8 \mathrm{~Hz}), 67.0,63.5(\mathrm{t}, J=6.4 \mathrm{~Hz}), 36.5(\mathrm{~d}, J=6.2 \mathrm{~Hz}), 30.6,27.2,26.9,22.0,16.0(\mathrm{~d}, J=7.1 \mathrm{~Hz})$, 13.8. IR (thin film) $v_{\max } 3472,2869,2242,1265 \mathrm{~cm}^{-1}$. MS (EI): $\mathrm{m} / z(\%) 304\left(\mathrm{M}^{+}\right), 275,155(100)$. HRMS: Calculated for $\mathrm{C}_{15} \mathrm{H}_{29} \mathrm{O}_{4} \mathrm{P}: 304.1803$; Found: 304.1800 . 


\section{General Procedure for Cu-Catalyzed Cross-Coupling of Propargyl Phosphates 2 with Polyfluoroarenes 1}

To a septum capped $25 \mathrm{~mL}$ of Schlenck tube were added CuOAc (10 mol\%), $t \mathrm{BuOLi}$ (2.4 equiv) under $\mathrm{N}_{2}$, followed by THF (2.0 mL), propargyl phosphate 2 ( $0.6 \mathrm{mmol}, 1.0$ equiv), and polyfluoroarene $\mathbf{1}$ (3.0 or 4.0 equiv). The tube was screw capped and put into an oil bath (preheated to $80{ }^{\circ} \mathrm{C}$ ). After stirring for $12 \mathrm{~h}$, the reaction mixture was cooled to room temperature, and diluted with ethyl acetate, washed with brine, dried over $\mathrm{Na}_{2} \mathrm{SO}_{4}$, filtered and concentrated. The residue was purified with silica gel chromatography to provide pure product 3 or 4 .

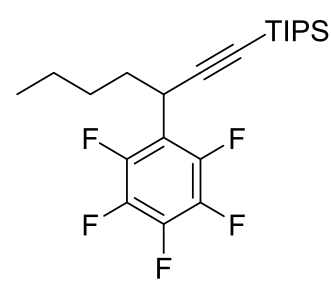

Triisopropyl (3-(perfluorophenyl)hept-1-yn-1-yl)silane (3a). The product (213 $\mathrm{mg}, 85 \%$ yield) as a colorless oil was purified with silica gel chromatography (Petroleum ether (100\%)). ${ }^{1} \mathrm{H}$ NMR (400 $\left.\mathrm{MHz}, \mathrm{CDCl}_{3}\right) \delta 4.02(\mathrm{t}, J=7.6 \mathrm{~Hz}, 1 \mathrm{H}), 2.06-1.96(\mathrm{~m}, 1 \mathrm{H}), 1.81-1.73(\mathrm{~m}, 1 \mathrm{H}), 1.49-1.34(\mathrm{~m}, 4 \mathrm{H})$, 1.12-0.94 (m, 21H), $0.90(\mathrm{t}, J=6.5 \mathrm{~Hz}, 3 \mathrm{H}) .{ }^{19} \mathrm{~F}$ NMR $\left(376 \mathrm{MHz}, \mathrm{CDCl}_{3}\right) \delta-142.4(\mathrm{dd}, J=21.4,6.8$ $\mathrm{Hz}, 2 \mathrm{~F}),-157.1(\mathrm{t}, J=21.0 \mathrm{~Hz}, 1 \mathrm{~F}),-162.8(\mathrm{td}, J=21.3,7.1 \mathrm{~Hz}, 2 \mathrm{~F}) .{ }^{13} \mathrm{C} \mathrm{NMR}\left(126 \mathrm{MHz}, \mathrm{CDCl}_{3}\right) \delta$ $145.1(\mathrm{dm}, J=252.1 \mathrm{~Hz}), 140.32(\mathrm{dm}, J=252.7 \mathrm{~Hz}), 137.79(\mathrm{dm}, J=252.0 \mathrm{~Hz}), 115.86(\mathrm{td}, J=15.9$, 3.9 Hz), 106.0, 82.9, 35.1, 29.9, 28.1, 22.3, 18.7, 14.0, 11.4. IR (thin film) $v_{\max } 3853,2943,2866,2174$, $1522 \mathrm{~cm}^{-1}$. MS (EI): $\mathrm{m} / z(\%) 418\left(\mathrm{M}^{+}\right), 419[\mathrm{M}+\mathrm{H}]^{+}, 375$ (100). HRMS: Calculated for $\mathrm{C}_{22} \mathrm{H}_{31} \mathrm{~F}_{5} \mathrm{Si}$ : 418.2115; Found: 418.2120.

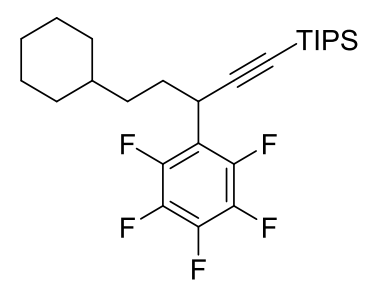

(5-Cyclohexyl-3-(perfluorophenyl)pent-1-yn-1-yl)triisopropylsilane (3b). The product (235 mg, 83\% yield) as a colorless oil was purified with silica gel chromatography (Petroleum ether $(100 \%)$ ). ${ }^{1} \mathrm{H}$ 
NMR (400 MHz, $\left.\mathrm{CDCl}_{3}\right) \delta 3.97(\mathrm{t}, J=7.7 \mathrm{~Hz}, 1 \mathrm{H}), 1.98-1.93(\mathrm{~m}, 1 \mathrm{H}), 1.79-1.63(\mathrm{~m}, 5 \mathrm{H}), 1.46-1.40$ $(\mathrm{m}, 1 \mathrm{H}), 1.26-1.14(\mathrm{~m}, 5 \mathrm{H}), 1.11-0.96(\mathrm{~m}, 21 \mathrm{H}), 0.91-0.80(\mathrm{~m}, 3 \mathrm{H}) .{ }^{19} \mathrm{~F} \mathrm{NMR}\left(376 \mathrm{MHz}, \mathrm{CDCl}_{3}\right) \delta-$ $142.0(\mathrm{dd}, J=21.7,7.5 \mathrm{~Hz}, 2 \mathrm{~F}),-156.8(\mathrm{t}, J=21.0 \mathrm{~Hz}, 1 \mathrm{~F}),-162.5(\mathrm{td}, J=21.7,7.8 \mathrm{~Hz}, 2 \mathrm{~F}) .{ }^{13} \mathrm{C}$ NMR $\left(126 \mathrm{MHz}, \mathrm{CDCl}_{3}\right) \delta 144.8(\mathrm{dm}, J=248.3 \mathrm{~Hz}), 140.1(\mathrm{dm}, J=253.1 \mathrm{~Hz}), 137.5(\mathrm{dm}, J=255.5 \mathrm{~Hz})$, $115.7(\mathrm{tm}, J=15.6 \mathrm{~Hz}), 105.8,82.7,37.2,35.2,33.4,33.1,32.7,26.6,26.3,18.4$, 11.2. IR (thin film) $v_{\max } 2926,2866,2175,1653 \mathrm{~cm}^{-1}$. MS (EI): $m / z(\%) 472\left(\mathrm{M}^{+}\right), 473[\mathrm{M}+\mathrm{H}]^{+}, 429$ (100). HRMS: Calculated for $\mathrm{C}_{26} \mathrm{H}_{37} \mathrm{~F}_{5} \mathrm{Si}$ : 472.2585 ; Found: 472.2580 .

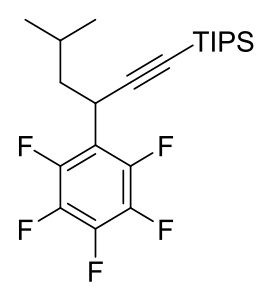

Triisopropyl(5-methyl-3-(perfluorophenyl)hex-1-yn-1-yl)silane (3c). CuOAc (20 mol\%) was used. The product (221 mg, 88\% yield) as a colorless oil was purified with silica gel chromatography (Petroleum ether (100\%)). ${ }^{1} \mathrm{H}$ NMR (400 MHz, $\left.\mathrm{CDCl}_{3}\right) \delta 4.15(\mathrm{t}, J=7.8 \mathrm{~Hz}, 1 \mathrm{H}), 1.98-1.91(\mathrm{~m}, 1 \mathrm{H})$, 1.83-1.73 (m, 1H), 1.63-1.56 (m, 1H), 1.10-1.06 (m, 21H), 0.99-0.97 (m, 6H) . ${ }^{19} \mathrm{~F}$ NMR (376 MHz, $\left.\mathrm{CDCl}_{3}\right) \delta-142.4(\mathrm{dd}, J=21.2,7.0 \mathrm{~Hz}, 2 \mathrm{~F}),-157.1(\mathrm{t}, J=20.8 \mathrm{~Hz}, 1 \mathrm{~F}),-162.7(\mathrm{td}, J=21.5,7.7 \mathrm{~Hz}$, 2F). ${ }^{13} \mathrm{C} \mathrm{NMR}\left(126 \mathrm{MHz}, \mathrm{CDCl}_{3}\right) \delta 144.8(\mathrm{dm}, J=252.4 \mathrm{~Hz}), 140.08(\mathrm{dm}, J=252.6 \mathrm{~Hz}), 137.56(\mathrm{dm}$, $J=252.6 \mathrm{~Hz}), 115.77(\mathrm{td}, J=16.2,4.4 \mathrm{~Hz}), 105.7,82.6,44.0,26.2,26.0,22.3,21.6,18.4,11.2 . \mathrm{IR}$ (thin film) $v_{\max } 2959,2867,2174,1521 \mathrm{~cm}^{-1} . \mathrm{MS}(\mathrm{EI}): \mathrm{m} / \mathrm{z}(\%) 418\left(\mathrm{M}^{+}\right), 419[\mathrm{M}+\mathrm{H}]^{+}, 375(100)$. HRMS: Calculated for $\mathrm{C}_{22} \mathrm{H}_{31} \mathrm{~F}_{5} \mathrm{Si}$ : 418.2115; Found: 418.2120 .

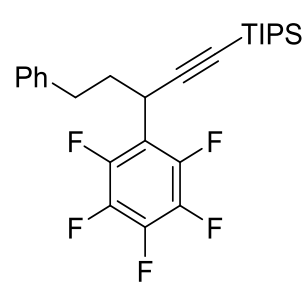

Triisopropyl(3-(perfluorophenyl)-5-phenylpent-1-yn-1-yl)silane (3d). The product (240 mg, 86\% yield) as a white solid (m.p. $36{ }^{\circ} \mathrm{C}$ ) was purified with silica gel chromatography (Petroleum ether/ EtOAc $=50: 1) .{ }^{1} \mathrm{H}$ NMR $\left(500 \mathrm{MHz}, \mathrm{CDCl}_{3}\right) \delta 7.28-7.25(\mathrm{~m}, 2 \mathrm{H}), 7.18-7.16(\mathrm{~m}, 3 \mathrm{H}), 4.03(\mathrm{t}, J=7.6$ $\mathrm{Hz}, 1 \mathrm{H}), 3.00-2.87(\mathrm{~m}, 1 \mathrm{H}), 2.75-2.69(\mathrm{~m}, 1 \mathrm{H}), 2.34-2.27(\mathrm{~m}, 1 \mathrm{H}), 2.08-2.00(\mathrm{~m}, 1 \mathrm{H}), 1.25-0.93(\mathrm{~m}$, 
21H). ${ }^{19} \mathrm{~F} \mathrm{NMR}\left(376 \mathrm{MHz}, \mathrm{CDCl}_{3}\right) \delta-142.9(\mathrm{dd}, J=20.7,6.9 \mathrm{~Hz}, 2 \mathrm{~F}),-158.2(\mathrm{t}, J=20.4 \mathrm{~Hz}, 1 \mathrm{~F}),-$ $163.9(\mathrm{dt}, J=21.3,7.2 \mathrm{~Hz}, 2 \mathrm{~F}) .{ }^{13} \mathrm{C} \mathrm{NMR}\left(126 \mathrm{MHz}, \mathrm{CDCl}_{3}\right) \delta 144.9(\mathrm{dm}, J=245.4 \mathrm{~Hz}), 140.5,140.3$ $(\mathrm{dm}, J=253.2 \mathrm{~Hz}), 137.6(\mathrm{dm}, J=252.3 \mathrm{~Hz}), 128.5,128.4,126.2,115.3(\mathrm{tm}, J=15.5 \mathrm{~Hz}), 105.3$, 83.6, 36.8, 33.8, 27.5, 18.6, 11.3. IR (KBr disk) $v_{\max } 3028,2943,2890,2865,2175,2723,1506 \mathrm{~cm}^{-1}$. MS (EI): $m / z(\%) 423\left[\left(\mathrm{M}-\mathrm{C}_{3} \mathrm{H}_{7}\right)^{+}, 100\right], 395,381$. HRMS: Calculated for $\mathrm{C}_{23} \mathrm{H}_{24} \mathrm{~F}_{5} \mathrm{Si}$ : 423.1567; Found: 423.1563. Anal. Calcd. For $\mathrm{C}_{23} \mathrm{H}_{24} \mathrm{~F}_{5} \mathrm{Si}$ : C, 66.93; H, 6.70; Found: C, 66.89; H, 6.61.

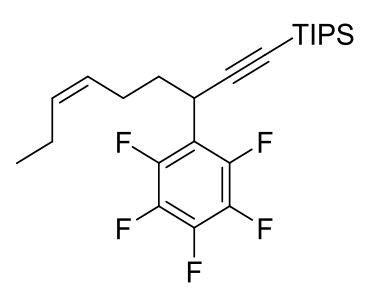

(Z)-Triisopropyl(3-(perfluorophenyl)non-6-en-1-yn-1-yl)silane (3e). The product (186 mg, 70\% yield) as a colorless oil was purified with silica gel chromatography (Petroleum ether/ EtOAc = 100:1). ${ }^{1} \mathrm{H}$ NMR $\left(500 \mathrm{MHz}, \mathrm{CDCl}_{3}\right) \delta 5.45(\mathrm{ddd}, J=12.8,9.7,7.3 \mathrm{~Hz}, 1 \mathrm{H}), 5.29(\mathrm{ddd}, J=10.7,9.7,7.3 \mathrm{~Hz}$, $1 \mathrm{H}), 4.05(\mathrm{dd}, J=9.0,6.4 \mathrm{~Hz}, 1 \mathrm{H}), 2.28-2.19(\mathrm{~m}, 2 \mathrm{H}), 2.10-2.02(\mathrm{~m}, 3 \mathrm{H}), 1.78-1.71(\mathrm{~m}, 1 \mathrm{H}), 1.12-$ $1.00(\mathrm{~m}, 21 \mathrm{H}), 0.96(\mathrm{t}, J=7.5 \mathrm{~Hz}, 3 \mathrm{H}) .{ }^{19} \mathrm{~F} \mathrm{NMR}\left(376 \mathrm{MHz}, \mathrm{CDCl}_{3}\right) \delta-142.0(\mathrm{dd}, J=21.5,7.0 \mathrm{~Hz}$, 2F), $-156.7(\mathrm{t}, J=21.0 \mathrm{~Hz}, 1 \mathrm{~F}),-162.4(\mathrm{td}, J=21.6,7.6 \mathrm{~Hz}, 2 \mathrm{~F}) .{ }^{13} \mathrm{C} \mathrm{NMR}\left(126 \mathrm{MHz}, \mathrm{CDCl}_{3}\right) \delta 144.8$ $(\mathrm{dm}, J=248.9 \mathrm{~Hz}), 140.1(\mathrm{dm}, J=258.0 \mathrm{~Hz}), 137.5(\mathrm{dm}, J=245.3 \mathrm{~Hz}), 133.5,126.8,115.5(\mathrm{td}, J=$ 16.3, $3.5 \mathrm{~Hz}), 105.5,83.0,35.1,27.3,25.1,20.6,18.5,14.2,11.2$. IR (thin film) $v_{\max } 3008,2944,2867$,

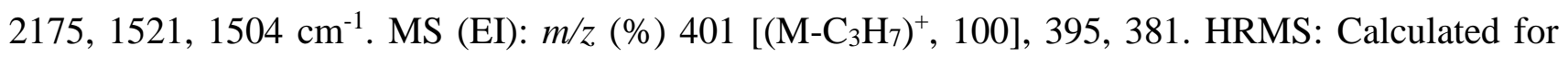
$\mathrm{C}_{21} \mathrm{H}_{26} \mathrm{~F}_{5} \mathrm{Si}: 401.1724$; Found: 401.1717.

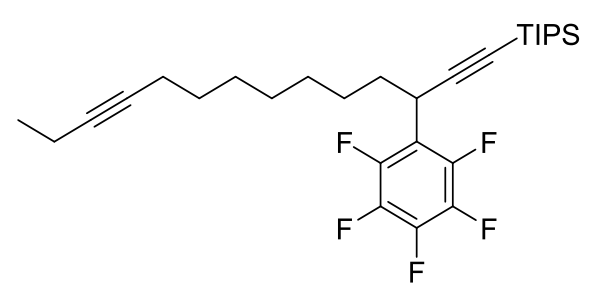

Triisopropyl(3-(perfluorophenyl)tetradeca-1,11-diyn-1-yl)silane (3f). The product (280 mg, $91 \%$ yield) as a colorless oil was purified with silica gel chromatography (Petroleum ether (100\%)). ${ }^{1} \mathrm{H}$ NMR (400 MHz, $\left.\mathrm{CDCl}_{3}\right) \delta 4.01(\mathrm{t}, J=7.6 \mathrm{~Hz}, 1 \mathrm{H}), 2.18-2.11(\mathrm{~m}, 4 \mathrm{H}), 1.96-1.91(\mathrm{~m}, 2 \mathrm{H}), 1.74-1.70$ $(\mathrm{m}, 2 \mathrm{H}), 1.48-1.45(\mathrm{~m}, 4 \mathrm{H}), 1.33-1.26(\mathrm{~m}, 4 \mathrm{H}), 1.13-0.92(\mathrm{~m}, 24 \mathrm{H}) .{ }^{19} \mathrm{~F}$ NMR $\left(376 \mathrm{MHz}, \mathrm{CDCl}_{3}\right) \delta$ - 
$142.0(\mathrm{dd}, J=21.5,7.0 \mathrm{~Hz}, 2 \mathrm{~F}),-156.7(\mathrm{t}, J=21.0 \mathrm{~Hz}, 1 \mathrm{~F}),-162.4(\mathrm{td}, J=21.6,7.6 \mathrm{~Hz}, 2 \mathrm{~F}) .{ }^{13} \mathrm{C} \mathrm{NMR}$ $\left(126 \mathrm{MHz}, \mathrm{CDCl}_{3}\right) \delta 144.8(\mathrm{~d}, J=248.4 \mathrm{~Hz}), 140.0(\mathrm{~d}, J=253.2 \mathrm{~Hz}), 137.5(\mathrm{~d}, J=252.3 \mathrm{~Hz}), 115.6$ $(\mathrm{tm}, J=16.1 \mathrm{~Hz}), 105.7,82.7,81.6,79.4,35.1,29.7,29.1,28.9,28.8,28.7,27.8,27.5,18.5,14.4,12.4$, 11.2. IR (thin film) $v_{\max } 2939,2865,2175,1504 \mathrm{~cm}^{-1}$. MS (EI): $m / z(\%) 512\left(\mathrm{M}^{+}\right), 469,427,73(100)$. HRMS: Calculated for $\mathrm{C}_{29} \mathrm{H}_{41} \mathrm{~F}_{5} \mathrm{Si}$ : 512.2898; Found: 512.2892.

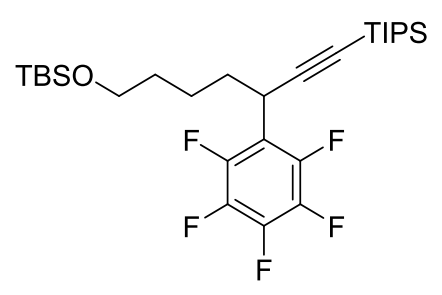

tert-Butyldimethyl((5-(perfluorophenyl)-7-(triisopropylsilyl)hept-6-yn-yl)oxy)silane (3g). The product (256 mg, 78\% yield) as a colorless oil was purified with silica gel chromatography (Petroleum ether/ EtOAc = 50:1). ${ }^{1} \mathrm{H}$ NMR $\left(400 \mathrm{MHz}, \mathrm{CDCl}_{3}\right) \delta 4.03(\mathrm{t}, J=7.6 \mathrm{~Hz}, 1 \mathrm{H}), 3.60(\mathrm{t}, J=6.0 \mathrm{~Hz}, 2 \mathrm{H})$, 2.04-1.95 (m, 1H), 1.80- $1.76(\mathrm{~m}, 1 \mathrm{H}), 1.59-1.54(\mathrm{~m}, 3 \mathrm{H}), 1.47-1.40(\mathrm{~m}, 1 \mathrm{H}), 1.08-1.01(\mathrm{~m}, 21 \mathrm{H}), 0.87$ $(\mathrm{s}, 9 \mathrm{H}), 0.03(\mathrm{~s}, 6 \mathrm{H}) .{ }^{19} \mathrm{~F}$ NMR $\left(376 \mathrm{MHz}, \mathrm{CDCl}_{3}\right)-141.9(\mathrm{dd}, J=21.5,7.3 \mathrm{~Hz}, 2 \mathrm{~F}),-156.7$ (t, $J=20.9$ $\mathrm{Hz}, 2 \mathrm{~F}),-162.5(\mathrm{td}, J=21.7,7.8 \mathrm{~Hz}, 2 \mathrm{~F}) .{ }^{13} \mathrm{C} \mathrm{NMR}\left(126 \mathrm{MHz}, \mathrm{CDCl}_{3}\right) 144.8(\mathrm{dm}, J=250.5 \mathrm{~Hz})$, $140.1(\mathrm{dm}, J=253.2 \mathrm{~Hz}), 137.5(\mathrm{dm}, J=251.6 \mathrm{~Hz}), 115.4(\mathrm{tm}, J=14.4 \mathrm{~Hz}), 105.6,82.7,62.8,35.0$, $32.1,27.9,25.9,24.0,18.5,18.3,11.2,-5.4$. IR (thin film) $v_{\max } 2944,2865,2175,1653,1504 \mathrm{~cm}^{-1}$. MS (MALDI): $m / z(\%) 549[\mathrm{M}+\mathrm{H}]^{+}, 532,520$. HRMS: Calculated for $\mathrm{C}_{28} \mathrm{H}_{46} \mathrm{~F}_{5} \mathrm{OSi}_{2}$ : 549.3002 ; Found: 549.2990.

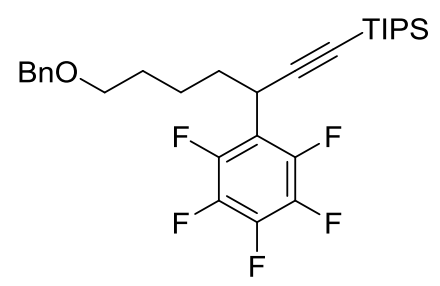

(7-(Benzyloxy)-3-(perfluorophenyl)hept-1-yn-1-yl)triisopropylsilane (3h). The product (255 mg, $81 \%$ yield) as a light yellow oil was purified with silica gel chromatography (Petroleum ether/ EtOAc = 50:1). ${ }^{1} \mathrm{H}$ NMR (400 MHz, $\left.\mathrm{CDCl}_{3}\right) \delta$ 7.35-7.24 (m, 5H), $4.48(\mathrm{~s}, 2 \mathrm{H}), 4.03(\mathrm{t}, J=7.6 \mathrm{~Hz}, 1 \mathrm{H}), 3.46$ $(\mathrm{t}, J=6.2 \mathrm{~Hz}, 2 \mathrm{H}), 2.02-1.95(\mathrm{~m}, 1 \mathrm{H}), 1.81-1.72(\mathrm{~m}, 1 \mathrm{H}), 1.69-1.57(\mathrm{~m}, 3 \mathrm{H}), 1.48-1.40(\mathrm{~m}, 1 \mathrm{H}), 1.08-$ $1.00(\mathrm{~m}, 21 \mathrm{H}) .{ }^{19} \mathrm{~F} \mathrm{NMR}\left(376 \mathrm{MHz}, \mathrm{CDCl}_{3}\right) \delta-142.0(\mathrm{dd}, J=21.5,7.4 \mathrm{~Hz}, 2 \mathrm{~F}),-156.6(\mathrm{t}, J=21.0 \mathrm{~Hz}$, 
1F), $-162.4(\mathrm{td}, J=21.7,7.8 \mathrm{~Hz}, 2 \mathrm{~F}) .{ }^{13} \mathrm{C} \mathrm{NMR}\left(126 \mathrm{MHz}, \mathrm{CDCl}_{3}\right) \delta 144.8(\mathrm{dm}, J=244.9 \mathrm{~Hz}), 140.1$ $(\mathrm{dm}, J=253.0 \mathrm{~Hz}), 138.5,137.6(\mathrm{dm}, J=252.7 \mathrm{~Hz}), 128.3,127.6,127.5,115.4(\mathrm{dd}, J=16.3,3.6 \mathrm{~Hz})$, 105.6, 82.9, 72.9, 69.9, 35.0, 29.1, 27.8, 24.3, 18.5, 11.2. IR (thin film) $v_{\max } 2943,2866,2174,1504$ $\mathrm{cm}^{-1}$. MS (MALDI): $\mathrm{m} / z$ (\%) $525[\mathrm{M}+\mathrm{H}]^{+}, 481,451$. HRMS: Calculated for $\mathrm{C}_{2} \mathrm{H}_{38} \mathrm{~F}_{5} \mathrm{OSi}$ : 525.2607; Found: 525.2595.

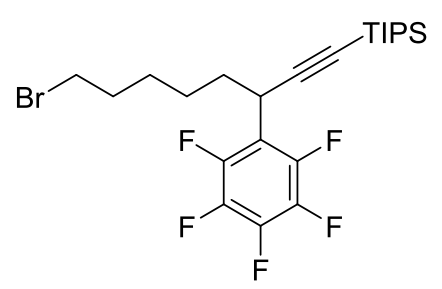

(8-Bromo-3-(perfluorophenyl)oct-1-yn-1-yl)triisopropylsilane (3i). The product (190 mg, 62\% yield) as a light yellow oil was purified with silica gel chromatography (Petroleum ether $(100 \%)) .{ }^{1} \mathrm{H}$ NMR (400 MHz, $\left.\mathrm{CDCl}_{3}\right) \delta 4.04(\mathrm{t}, J=7.6 \mathrm{~Hz}, 1 \mathrm{H}), 3.52(\mathrm{t}, J=6.6 \mathrm{~Hz}, 1 \mathrm{H}), 3.40(\mathrm{t}, J=6.7 \mathrm{~Hz}, 2 \mathrm{H})$, 2.04-1.95 (m, 1H), 1.90-1.83 (m, 1H), 1.81-1.72 (m, 1H), 1.58-1.41 (m, 4H), 1.05-0.90 (m, 21H). ${ }^{19} \mathrm{~F}$ NMR $\left(376 \mathrm{MHz}, \mathrm{CDCl}_{3}\right) \delta-142.0(\mathrm{dd}, J=21.0,6.5 \mathrm{~Hz}, 2 \mathrm{~F}),-156.6(\mathrm{t}, J=20.9 \mathrm{~Hz}, 1 \mathrm{~F}),-162.3(\mathrm{td}, J$ $=21.2,6.8 \mathrm{~Hz}, 2 \mathrm{~F}) .{ }^{13} \mathrm{C} \mathrm{NMR}\left(126 \mathrm{MHz}, \mathrm{CDCl}_{3}\right) \delta 144.8(\mathrm{dm}, J=248.3 \mathrm{~Hz}), 140.1(\mathrm{dm}, J=253.0$ Hz), $137.6(\mathrm{dm}, J=251.0 \mathrm{~Hz}), 115.4(\mathrm{td}, J=16.9,3.5 \mathrm{~Hz}), 105.4,83.0,34.9,33.4,32.5,27.8,27.5$, 26.7, 18.5, 11.2. IR (thin film) $v_{\max } 2943,2866,2176,1503 \mathrm{~cm}^{-1} . \mathrm{MS}(\mathrm{EI}): \mathrm{m} / z(\%) 469\left(\mathrm{M}-\mathrm{C}_{3} \mathrm{H}_{7}\right)^{+}$, 427, 167 (100). HRMS: Calculated for $\mathrm{C}_{20} \mathrm{H}_{25} \mathrm{BrF}_{5} \mathrm{Si}$ : 467.0829 ; Found: 467.0827.

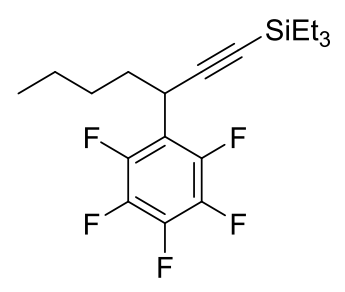

Triethyl(3-(perfluorophenyl)hept-1-yn-1-yl)silane (3j). The product (165 $\mathrm{mg}, 73 \%$ yield) as a colorless oil was purified with silica gel chromatography (Petroleum ether (100\%)). ${ }^{1} \mathrm{H}$ NMR (400 $\left.\mathrm{MHz}, \mathrm{CDCl}_{3}\right) \delta 4.00(\mathrm{t}, J=7.7 \mathrm{~Hz}, 1 \mathrm{H}), 1.98-1.93(\mathrm{~m}, 1 \mathrm{H}), 1.79-1.74(\mathrm{~m}, 1 \mathrm{H}), 1.48-1.41(\mathrm{~m}, 1 \mathrm{H})$, 1.39-1.26 (m, 3H), $0.97(\mathrm{t}, J=7.9 \mathrm{~Hz}, 9 \mathrm{H}), 0.90(\mathrm{t}, J=7.1 \mathrm{~Hz}, 3 \mathrm{H}), 0.58(\mathrm{q}, J=7.9 \mathrm{~Hz}, 6 \mathrm{H}) .{ }^{19} \mathrm{~F} \mathrm{NMR}$ $\left(376 \mathrm{MHz}, \mathrm{CDCl}_{3}\right) \delta-142.0(\mathrm{dd}, J=21.6,7.5 \mathrm{~Hz}, 2 \mathrm{~F}),-156.8(\mathrm{t}, J=20.9 \mathrm{~Hz}, 1 \mathrm{~F}),-162.5(\mathrm{td}, J=21.7$, $7.7 \mathrm{~Hz}, 2 \mathrm{~F}) .{ }^{13} \mathrm{C}$ NMR $\left(126 \mathrm{MHz}, \mathrm{CDCl}_{3}\right) \delta 144.8(\mathrm{dm}, J=251.4 \mathrm{~Hz}), 140.08(\mathrm{dm}, J=252.2 \mathrm{~Hz})$, 
$137.56(\mathrm{dm}, J=246.4 \mathrm{~Hz}), 115.45(\mathrm{tm}, J=16.8 \mathrm{~Hz}), 105.3,83.8,34.8,29.6,27.8,22.0,13.8,7.3,4.4$. IR (thin film) $v_{\max } 2958,2877,2175,1506 \mathrm{~cm}^{-1}$. MS (EI): $\mathrm{m} / z(\%) 376\left(\mathrm{M}^{+}\right), 347$ (100), 319. HRMS: Calculated for $\mathrm{C}_{19} \mathrm{H}_{25} \mathrm{~F}_{5} \mathrm{Si}$ : 376.1646; Found: 376.1646.

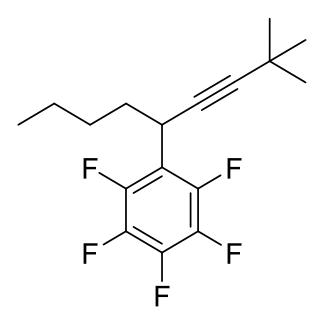

1-(2,2-Dimethylnon-3-yn-5-yl)-2,3,4,5,6-pentafluorobenzene (3k). The product (168 mg, 88\% yield) as a colorless oil was purified with silica gel chromatography (Petroleum ether (100\%)). ${ }^{1} \mathrm{H}$ NMR $(400$ $\left.\mathrm{MHz}, \mathrm{CDCl}_{3}\right) \delta 3.93(\mathrm{t}, J=7.7 \mathrm{~Hz}, 1 \mathrm{H}), 1.94-1.87(\mathrm{~m}, 1 \mathrm{H}), 1.79-1.68(\mathrm{~m}, 1 \mathrm{H}), 1.46-1.38(\mathrm{~m}, 1 \mathrm{H})$, 1.37-1.23 (m, 3H), $1.20(\mathrm{~s}, 9 \mathrm{H}), 0.90(\mathrm{t}, J=7.1 \mathrm{~Hz}, 3 \mathrm{H}) .{ }^{19} \mathrm{~F}$ NMR $\left(376 \mathrm{MHz}, \mathrm{CDCl}_{3}\right) \delta-143.5(\mathrm{dd}, J$ $=20.9,7.1 \mathrm{~Hz}, 2 \mathrm{~F}),-158.8(\mathrm{t}, J=20.3 \mathrm{~Hz}, 1 \mathrm{~F}),-164.1(\mathrm{td}, J=20.8,6.8 \mathrm{~Hz}, 2 \mathrm{~F}) .{ }^{13} \mathrm{C} \mathrm{NMR}(126 \mathrm{MHz}$, $\left.\mathrm{CDCl}_{3}\right) \delta 144.9(\mathrm{dm}, J=248.7 \mathrm{~Hz}), 139.9(\mathrm{dm}, J=252.3 \mathrm{~Hz}), 137.5(\mathrm{dm}, J=251.9 \mathrm{~Hz}), 116.4(\mathrm{td}, J$ $=16.0,4.4 \mathrm{~Hz}), 90.4,76.6,35.0,30.8,29.6,27.3,26.9,22.0,13.7$. IR (thin film) $v_{\max } 2968,2933,2867$, $1504 \mathrm{~cm}^{-1}$. MS (EI): $\mathrm{m} / \mathrm{z}(\%) 318\left(\mathrm{M}^{+}\right), 261,181(100)$. HRMS: Calculated for $\mathrm{C}_{17} \mathrm{H}_{19} \mathrm{~F}_{5}: 318.1407$; Found: 318.1408.

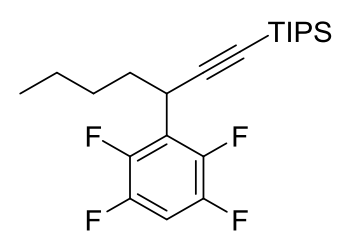

Triisopropyl(3-(2,3,5,6-tetrafluorophenyl)hept-1-yn-1-yl)silane (4a). 4.0 equiv of fluoroarene was used. The product (192 mg, 80\% yield) as a colorless oil was purified with silica gel chromatography (Petroleum ether (100\%)). ${ }^{1} \mathrm{H}$ NMR $\left(500 \mathrm{MHz}, \mathrm{CDCl}_{3}\right) \delta 6.95(\mathrm{tt}, J=9.6,7.3 \mathrm{~Hz}, 1 \mathrm{H}), 4.07(\mathrm{t}, J=7.7$ $\mathrm{Hz}, 1 \mathrm{H}), 2.00-1.97(\mathrm{~m}, 1 \mathrm{H}), 1.78-1.76(\mathrm{~m}, 1 \mathrm{H}), 1.50-1.48(\mathrm{~m}, 1 \mathrm{H}), 1.38-1.33(\mathrm{~m}, 3 \mathrm{H}), 1.09-1.01$ (m, 21H), $0.90(\mathrm{t}, J=7.2 \mathrm{~Hz}, 3 \mathrm{H}) .{ }^{19} \mathrm{~F}$ NMR $\left(376 \mathrm{MHz}, \mathrm{CDCl}_{3}\right) \delta-139.5(\mathrm{~m}, 2 \mathrm{~F}),-142.6(\mathrm{~m}, 2 \mathrm{~F}) .{ }^{13} \mathrm{C}$ NMR (126 MHz, $\left.\mathrm{CDCl}_{3}\right) \delta 146.2(\mathrm{~d}, J=181.4 \mathrm{~Hz}), 144.2(\mathrm{~d}, J=181.7 \mathrm{~Hz}), 121.4(\mathrm{t}, J=15.4 \mathrm{~Hz})$, 106.1, 104.3 (t, $J=22.6 \mathrm{~Hz}), 82.4,34.8,29.7,28.2,22.0,18.5,13.8,11.2$. IR (thin film) $v_{\max } 2944$, 2866, 2172, $1502 \mathrm{~cm}^{-1}$. MS (EI): $m / z(\%) 400\left(\mathrm{M}^{+}\right), 401[\mathrm{M}+\mathrm{H}]^{+}, 357$ (100). HRMS: Calculated for $\mathrm{C}_{22} \mathrm{H}_{32} \mathrm{~F}_{4} \mathrm{Si}: 400.2209$; Found: 400.2214. 
Gram-Scale Synthesis of 4a. To a septum capped $100 \mathrm{~mL}$ of Schlenck tube were added CuOAc (10 mol\%), $t$ BuOLi (2.4 equiv) under $\mathrm{N}_{2}$, followed by THF (15 mL). Propargyl phosphate 2a (4 mmol, 1.0 equiv), pentafluorobenzene ( $16 \mathrm{mmol}, 4.0$ equiv) were then added. The tube was screw capped and then conducted on oil bath (preheated to $80^{\circ} \mathrm{C}$ ). After stirring for $12 \mathrm{~h}$, the reaction mixture was cooled to room temperature, and diluted with ethyl acetate, washed with brine, dried over $\mathrm{Na}_{2} \mathrm{SO}_{4}$, filtered and concentrated. The residue was purified with silica gel chromatography (Petroleum ether (100\%) to provide pure $\mathbf{4 a}(1.2 \mathrm{~g}, 75 \%$ yield $)$.

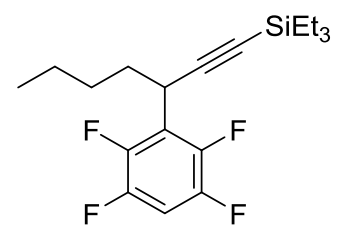

Triethyl(3-(2,3,5,6-tetrafluorophenyl)hept-1-yn-1-yl)silane (4b). 4.0 equiv of fluoroarene was used. The product (193 mg, 90\% yield) as a colorless oil was purified with silica gel chromatography (Petroleum ether (100\%)). ${ }^{1} \mathrm{H}$ NMR $\left(400 \mathrm{MHz}, \mathrm{CDCl}_{3}\right) \delta 6.96(\mathrm{tt}, J=9.6,7.3 \mathrm{~Hz}, 1 \mathrm{H}), 4.05(\mathrm{t}, J=7.7$ $\mathrm{Hz}, 1 \mathrm{H}), 1.99-1.96(\mathrm{~m}, 1 \mathrm{H}), 1.80-1.76(\mathrm{~m}, 1 \mathrm{H}), 1.47-1.43(\mathrm{~m}, 1 \mathrm{H}), 1.39-1.27(\mathrm{~m}, 3 \mathrm{H}), 0.97(\mathrm{t}, J=7.9$ $\mathrm{Hz}, 9 \mathrm{H}), 0.91-0.85(\mathrm{~m}, 4 \mathrm{H}), 0.58(\mathrm{q}, J=7.9 \mathrm{~Hz}, 5 \mathrm{H}) .{ }^{19} \mathrm{~F} \mathrm{NMR}\left(376 \mathrm{MHz}, \mathrm{CDCl}_{3}\right) \delta-139.5(\mathrm{~m}, 2 \mathrm{~F})$, $-142.6(\mathrm{~m}, 2 \mathrm{~F}) .{ }^{13} \mathrm{C} \mathrm{NMR}\left(126 \mathrm{MHz}, \mathrm{CDCl}_{3}\right) \delta 145.9(\mathrm{dm}, J=247.6 \mathrm{~Hz}), 144.5(\mathrm{dm}, J=252.2 \mathrm{~Hz})$, $121.3(\mathrm{t}, J=15.5 \mathrm{~Hz}), 105.6,104.3(\mathrm{t}, J=22.6 \mathrm{~Hz}), 83.5,34.7,29.6,28.2,22.1,13.8,7.3$, 4.4. IR (thin film) $v_{\max } 2958,2930,2173,1509 \mathrm{~cm}^{-1}$. MS (EI): $\mathrm{m} / z(\%) 358\left(\mathrm{M}^{+}\right), 359[\mathrm{M}+\mathrm{H}]^{+}, 329(100)$. HRMS: Calculated for $\mathrm{C}_{19} \mathrm{H}_{26} \mathrm{~F}_{4} \mathrm{Si}$ : 358.1740; Found: 358.1740 .<smiles></smiles>

Triisopropyl(5-phenyl-3-(2,3,5,6-tetrafluorophenyl)pent-1-yn-1-yl)silane $\quad$ (4c). 4.0 equiv of fluoroarene was used. The product (223 mg, 83\% yield) as a colorless oil was purified with silica gel chromatography (Petroleum ether (100\%)). ${ }^{1} \mathrm{H} \mathrm{NMR}\left(400 \mathrm{MHz}, \mathrm{CDCl}_{3}\right) \delta 7.28(\mathrm{t}, J=7.6 \mathrm{~Hz}, 2 \mathrm{H})$, 7.21-7.17 (m, 3H), 6.95 (tt, $J=9.5 \mathrm{~Hz}, 7.5 \mathrm{~Hz}, 1 \mathrm{H}), 4.10-4.06(\mathrm{~m}, 1 \mathrm{H}), 2.93-2.86(\mathrm{~m}, 1 \mathrm{H}), 2.76-2.69$ $(\mathrm{m}, 1 \mathrm{H}), 2.35-2.26(\mathrm{~m}, 1 \mathrm{H}), 2.20-2.01(\mathrm{~m}, 1 \mathrm{H}), 1.09-1.06(\mathrm{~m}, 21 \mathrm{H}) .{ }^{19} \mathrm{~F} \mathrm{NMR}\left(376 \mathrm{MHz}, \mathrm{CDCl}_{3}\right) \delta-$ 
$139.3(\mathrm{~m}, 2 \mathrm{~F}),-142.4(\mathrm{~m}, 2 \mathrm{~F}) .{ }^{13} \mathrm{C} \mathrm{NMR}\left(126 \mathrm{MHz}, \mathrm{CDCl}_{3}\right) \delta 145.9(\mathrm{dm}, J=247.9 \mathrm{~Hz}), 144.5(\mathrm{dm}$, $J=248.0 \mathrm{~Hz}), 140.7,128.5,128.4,126.2,121.0(\mathrm{t}, J=15.3 \mathrm{~Hz}), 105.5,104.5(\mathrm{t}, J=22.6 \mathrm{~Hz}), 83.3$, 36.6, 33.8, 27.9, 18.6, 11.2. IR (thin film) $v_{\max } 3066,2943,2177,1502 \mathrm{~cm}^{-1}$. MS (EI): $\mathrm{m} / z(\%) 448$ $\left(\mathrm{M}^{+}\right), 405$ (100), 363. HRMS: Calculated for $\mathrm{C}_{26} \mathrm{H}_{32} \mathrm{~F}_{4} \mathrm{Si}$ : 448.2209; Found: 448.2202.

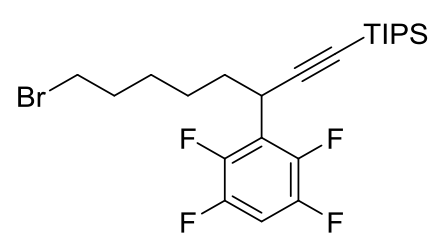

(8-Bromo-3-(2,3,5,6-tetrafluorophenyl)oct-1-yn-1-yl)triisopropylsilane (4d). $20 \mathrm{~mol} \%$ of CuOAc and 4.0 equiv of fluoroarene were used. The product ( $215 \mathrm{mg}, 73 \%$ yield) as a colorless oil was purified with silica gel chromatography (Petroleum ether (100\%)). ${ }^{1} \mathrm{H} \mathrm{NMR}\left(400 \mathrm{MHz}, \mathrm{CDCl}_{3}\right) \delta 6.97(\mathrm{tt}, J=$ 9.6, 7.3 Hz, 1H), 4.08 (t, $J=7.7 \mathrm{~Hz}, 1 \mathrm{H}), 3.40(\mathrm{t}, J=6.8 \mathrm{~Hz}, 2 \mathrm{H}), 2.05-1.96(\mathrm{~m}, 1 \mathrm{H}), 1.90-1.83(\mathrm{~m}$, 2H), 1.81-1.74 (m, 1H), 1.61-1.36 (m, 4H), 1.08-1.05(m, 21H). ${ }^{19} \mathrm{~F} \mathrm{NMR}\left(376 \mathrm{MHz}, \mathrm{CDCl}_{3}\right) \delta-139.3$ $(\mathrm{m}, 2 \mathrm{~F}),-142.5(\mathrm{~m}, 2 \mathrm{~F}) .{ }^{13} \mathrm{C} \mathrm{NMR}\left(126 \mathrm{MHz}, \mathrm{CDCl}_{3}\right) \delta 146.2(\mathrm{dm}, J=183.7 \mathrm{~Hz}), 144.2(\mathrm{dm}, J=$ $178.9 \mathrm{~Hz}), 121.1(\mathrm{t}, J=15.4 \mathrm{~Hz}), 105.7,104.4(\mathrm{t}, J=22.6 \mathrm{~Hz}), 82.8,34.8,33.5,32.5,28.2,27.5,26.7$, 18.5, 11.2. IR (thin film) $v_{\max } 3080,2943,2866,2175,1503 \mathrm{~cm}^{-1} . \mathrm{MS}(\mathrm{EI}): \mathrm{m} / z(\%) 449\left(\mathrm{M}-\mathrm{C}_{3} \mathrm{H}_{7}\right)^{+}$, 451, 370. HRMS: Calculated for $\mathrm{C}_{20} \mathrm{H}_{26} \mathrm{~F}_{4} \mathrm{SiBr}$ : 449.0923; Found: 449.0933.

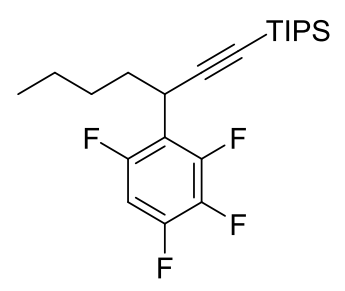

Triisopropyl(3-(2,3,4,6-tetrafluorophenyl)hept-1-yn-1-yl)silane (4e). 20 mol\% of CuOAc and 4.0 equiv of fluoroarene were used. The product (194 mg, $81 \%$ yield) as a colorless oil was purified with silica gel chromatography (Petroleum ether (100\%)). ${ }^{1} \mathrm{H}$ NMR $\left(400 \mathrm{MHz}, \mathrm{CDCl}_{3}\right) \delta 6.78-6.72(\mathrm{~m}, 1 \mathrm{H})$, $4.01(\mathrm{t}, J=7.7 \mathrm{~Hz}, 1 \mathrm{H}), 2.01-1.95(\mathrm{~m}, 1 \mathrm{H}), 1.80-1.73(\mathrm{~m}, 1 \mathrm{H}), 1.54-1.48(\mathrm{~m}, 1 \mathrm{H}), 1.41-1.33(\mathrm{~m}, 3 \mathrm{H})$, $1.12-1.00(\mathrm{~m}, 21 \mathrm{H}), 0.91(\mathrm{t}, J=7.1 \mathrm{~Hz}, 3 \mathrm{H}) .{ }^{19} \mathrm{~F} \mathrm{NMR}\left(376 \mathrm{MHz}, \mathrm{CDCl}_{3}\right) \delta-116.9(\mathrm{t}, J=9.9 \mathrm{~Hz}, 1 \mathrm{~F})$, $-134.4(\mathrm{~d}, J=20.0 \mathrm{~Hz}, 1 \mathrm{~F}),-134.8(\mathrm{ddd}, J=20.8,9.3,5.2 \mathrm{~Hz}, 1 \mathrm{~F}),-165.2(\mathrm{~m}, 1 \mathrm{~F}) .{ }^{13} \mathrm{C}$ NMR $(126$ $\left.\mathrm{MHz}, \mathrm{CDCl}_{3}\right) \delta 154.9(\mathrm{dm}, J=247.1 \mathrm{~Hz}), 149.7(\mathrm{dm}, J=246.6 \mathrm{~Hz}), 149.4(\mathrm{dm}, J=250.0 \mathrm{~Hz}), 137.3$ 
$(\mathrm{dm}, J=253.7 \mathrm{~Hz}), 115.54(\mathrm{tm}, J=17.2 \mathrm{~Hz}), 106.6,100.6(\mathrm{~m}), 81.9,34.8,29.7,27.7,22.0,18.5,13.8$, 11.2. IR (thin film) $v_{\max } 2943,2866,2173,1640 \mathrm{~cm}^{-1}$. MS (EI): $m / z(\%) 400\left(\mathrm{M}^{+}\right), 401[\mathrm{M}+\mathrm{H}]^{+}, 357$ (100). HRMS: Calculated for $\mathrm{C}_{22} \mathrm{H}_{32} \mathrm{~F}_{4} \mathrm{Si}$ : 400.2209; Found: 400.2212.<smiles>Fc1cc(F)c(CCc2ccccc2)c(C(C#C[AlH2])CCc2ccccc2)c1F</smiles>

Triisopropyl(5-phenyl-3-(2,3,4,6-tetrafluorophenyl)pent-1-yn-1-yl)silane $\quad(4 f) . \quad 4.0$ equiv of fluoroarene was used. The product (188 mg, 70\% yield) as a light yellow oil was purified with silica gel chromatography (Petroleum ether/ EtOAc 100:1). ${ }^{1} \mathrm{H}$ NMR (400 MHz, $\left.\mathrm{CDCl}_{3}\right) \delta$ 7.30-7.27 (m, 2H), 7.22-7.18 (m, 3H), 6.77-6.70 (m, 1H), 4.03-3.99 (m, 1H), 2.93-2.85 (m, 1H), 2.76-2.68 (m, 1H), 2.33-2.24 (m, 1H), 2.08-1.99 (m, 1H), $1.20-0.94(\mathrm{~m}, 21 \mathrm{H}) .{ }^{19} \mathrm{~F} \mathrm{NMR}\left(376 \mathrm{MHz}, \mathrm{CDCl}_{3}\right) \delta-116.6(\mathrm{t}$, $J=10.3 \mathrm{~Hz}, 1 \mathrm{~F}),-134.2(\mathrm{~d}, J=20.5 \mathrm{~Hz}, 1 \mathrm{~F}),-134.3(\mathrm{~m}, 1 \mathrm{~F}),-165.0(\mathrm{tdd}, J=20.9,10.9,6.0 \mathrm{~Hz}, 1 \mathrm{~F})$. ${ }^{13} \mathrm{C}$ NMR $\left(126 \mathrm{MHz} \mathrm{CDCl}_{3}\right) \delta 154.9(\mathrm{dm}, J=251.9 \mathrm{~Hz}), 149.6(\mathrm{dm}, J=251.8 \mathrm{~Hz}), 149.5(\mathrm{dm}, J=$ $249.1 \mathrm{~Hz}), 140.8,137.3(\mathrm{dm}, J=243.2 \mathrm{~Hz}), 128.5,128.4,126.1,115.2(\mathrm{tm}, J=17.2 \mathrm{~Hz}), 106.1,100.8$ (ddd, $J=28.6,21.1,3.6 \mathrm{~Hz}$ ), 82.8, 36.8, 33.8, 27.4, 18.6, 11.3. IR (thin film) $v_{\max } 3028,2943,2174$, $1518 \mathrm{~cm}^{-1}$. MS (EI): $\mathrm{m} / \mathrm{z}(\%) 448\left(\mathrm{M}^{+}\right), 405$ (100), 363. HRMS: Calculated for $\mathrm{C}_{26} \mathrm{H}_{32} \mathrm{~F}_{4} \mathrm{Si}$ : 448.2209; Found: 448.2206.

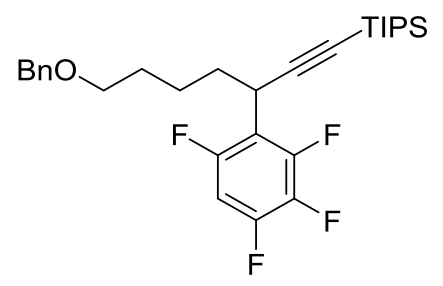

(7-(Benzyloxy)-3-(2,3,4,6-tetrafluorophenyl)hept-1-yn-1-yl)triisopropylsilane (4g). 4.0 equiv of fluoroarene was used. The product (216 mg, 71\% yield) as a light yellow oil was purified with silica gel chromatography (Petroleum ether/ EtOAc = 30:1). ${ }^{1} \mathrm{H}$ NMR $\left(400 \mathrm{MHz}, \mathrm{CDCl}_{3}\right)$ 7.36-7.31 (m, 4H), 7.30-7.24 (m, 1H), 6.78-6.71 (m, 1H), 4.52-4.40 (m, 2H), $4.02(\mathrm{t}, J=7.6 \mathrm{~Hz}, 1 \mathrm{H}), 3.53-3.46(\mathrm{~m}, 2 \mathrm{H})$, 2.05-1.97 (m, 1H), $1.82-1.74(\mathrm{~m}, 1 \mathrm{H}), 1.71-1.62(\mathrm{~m}, 3 \mathrm{H}), 1.50-1.43(\mathrm{~m}, 1 \mathrm{H}), 1.10-0.90(\mathrm{~m}, 21 \mathrm{H}) .{ }^{19} \mathrm{~F}$ NMR $\left(376 \mathrm{MHz}, \mathrm{CDCl}_{3}\right) \delta-116.8(\mathrm{t}, J=10.3 \mathrm{~Hz}),-134.3(\mathrm{dd}, J=20.6,3.9 \mathrm{~Hz}),-134.6(\mathrm{~m}),-165.1$ 
$(\operatorname{tdd}, J=21.0,10.9,6.0 \mathrm{~Hz}) .{ }^{13} \mathrm{C} \mathrm{NMR}\left(126 \mathrm{MHz}, \mathrm{CDCl}_{3}\right) \delta 154.9(\mathrm{dm}, J=247.6 \mathrm{~Hz}), 149.7(\mathrm{dm}, J=$ $246.1 \mathrm{~Hz}), 149.4(\mathrm{dm}, J=235.1 \mathrm{~Hz}), 138.6,137.3(\mathrm{dm}, J=248.9 \mathrm{~Hz}), 128.3,127.6,127.5,115.4(\mathrm{tm}$, $J=19.3 \mathrm{~Hz}), 106.4,100.8(\mathrm{ddd}, J=28.8,21.2,3.6 \mathrm{~Hz}), 82.2,72.9$, 70.0, 35.0, 29.1, 27.7, 24.3, 18.5, 11.2. IR (thin film) $v_{\max } 2985,1742,1654,1374 \mathrm{~cm}^{-1}$. MS (EI): $\mathrm{m} / z(\%) 463\left[\mathrm{M}_{-} \mathrm{C}_{3} \mathrm{H}_{7}\right]^{+}, 464,421$. HRMS: Calculated for $\mathrm{C}_{26} \mathrm{H}_{31} \mathrm{OF}_{4} \mathrm{Si}$ : 463.2080; Found: 463.2075 .

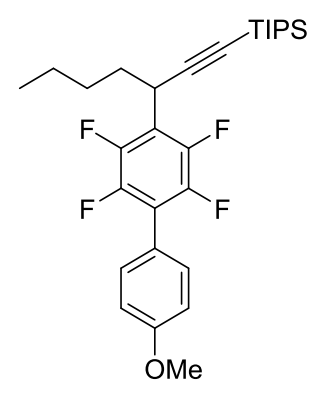

Triisopropyl(3-(2,3,5,6-tetrafluoro-4'-methoxy-[1,1'-biphenyl]-4-yl)hept-1-yn-1-yl)silane (4h). $20 \mathrm{~mol} \%$ of CuOAc and 3.0 equiv of fluoroarene were used, the reaction was conducted at $100{ }^{\circ} \mathrm{C}$. The product (246 mg, 81\% yield) as a colorless oil was purified with silica gel chromatography $($ Petroleum ether $/$ EtOAc $=100: 1) .{ }^{1} \mathrm{H}$ NMR $\left(500 \mathrm{MHz}, \mathrm{CDCl}_{3}\right) \delta 7.42(\mathrm{~d}, J=8.6 \mathrm{~Hz}, 2 \mathrm{H}), 7.03-7.01$ $(\mathrm{m}, 2 \mathrm{H}), 4.10(\mathrm{t}, J=7.7 \mathrm{~Hz}, 1 \mathrm{H}), 3.87(\mathrm{~s}, 3 \mathrm{H}), 2.05-1.98(\mathrm{~m}, 1 \mathrm{H}), 1.85-1.78(\mathrm{~m}, 1 \mathrm{H}), 1.57-1.50(\mathrm{~m}$, 1H), 1.41-1.36 (m, 3H), $1.10-1.04(\mathrm{~m}, 21 \mathrm{H}), 0.92(\mathrm{t}, J=7.1 \mathrm{~Hz}, 3 \mathrm{H}) .{ }^{19} \mathrm{~F} \mathrm{NMR}\left(376 \mathrm{MHz}, \mathrm{CDCl}_{3}\right)$ $143.2(\mathrm{dd}, J=22.2,12.2 \mathrm{~Hz}, 2 \mathrm{~F}),-145.1(\mathrm{dd}, J=22.3,12.2 \mathrm{~Hz}, 2 \mathrm{~F}) .{ }^{13} \mathrm{C} \mathrm{NMR}\left(126 \mathrm{MHz}, \mathrm{CDCl}_{3}\right) \delta$ 160.0, $145.2(\mathrm{dm}, J=130.1 \mathrm{~Hz}), 143.3(\mathrm{dm}, J=126.0 \mathrm{~Hz}), 131.4,119.6,118.9$, 114.1, 110.0, 106.3, 82.3, 55.3, 34.97, 29.7, 28.2, 22.1, 18.6, 13.9, 11.3. IR (thin film) $v_{\max } 3358,2942,2865,2172,1480$ $\mathrm{cm}^{-1}$. MS (DART): $\mathrm{m} / \mathrm{z}(\%) 507[\mathrm{M}+\mathrm{H}]^{+}$. HRMS: Calculated for $\mathrm{C}_{29} \mathrm{H}_{39} \mathrm{~F}_{4} \mathrm{OSi}$ : 507.2701; Found: 507.2702 .

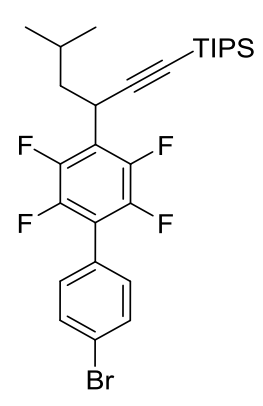

(3-(4'-Bromo-2,3,5,6-tetrafluoro-[1,1'-biphenyl]-4-yl)-5-methylhex-1-yn-1-yl)triisopropylsilane 
(4i). 3.0 equiv of fluoroarene was used. The product ( $283 \mathrm{mg}, 85 \%$ yield) as a colorless oil was purified with silica gel chromatography (Petroleum ether (100\%)). ${ }^{1} \mathrm{H}$ NMR (400 MHz, $\left.\mathrm{CDCl}_{3}\right) \delta 7.64-7.62$ $(\mathrm{m}, 2 \mathrm{H}), 7.36(\mathrm{~d}, J=8.3 \mathrm{~Hz}, 2 \mathrm{H}), 4.20(\mathrm{t}, J=7.9 \mathrm{~Hz}, 1 \mathrm{H}), 1.99-1.93(\mathrm{~m}, 1 \mathrm{H}), 1.84-1.76(\mathrm{~m}, 1 \mathrm{H}), 1.67-$ $1.61(\mathrm{~m}, 1 \mathrm{H}), 1.09-1.06(\mathrm{~m}, 21 \mathrm{H}), 0.99(\mathrm{~d}, J=6.6,3 \mathrm{H}), 0.98(\mathrm{~d}, J=6.6,3 \mathrm{H}) .{ }^{19} \mathrm{~F}$ NMR $(376 \mathrm{MHz}$, $\left.\mathrm{CDCl}_{3}\right)-142.6(\mathrm{dd}, J=21.9,12.3 \mathrm{~Hz}, 2 \mathrm{~F}),-144.5(\mathrm{dd}, J=22.1,12.3 \mathrm{~Hz}, 2 \mathrm{~F}) .{ }^{13} \mathrm{C}$ NMR $(126 \mathrm{MHz}$, $\left.\mathrm{CDCl}_{3}\right) \delta 145.3(\mathrm{dm}, J=150.9 \mathrm{~Hz}), 143.3(\mathrm{dm}, J=148.9 \mathrm{~Hz}), 131.8,131.7,126.4,123.5,120.2(\mathrm{t}, J$ $=15.8 \mathrm{~Hz}), 117.9(\mathrm{t}, J=17.2 \mathrm{~Hz}), 106.0,82.5,44.0,26.4,26.2,22.5,21.8,18.5,11.2 . \mathrm{IR}$ (thin film) $v_{\max } 2959,2867,2173,1742,1478 \mathrm{~cm}^{-1}$. MS (DART): $\mathrm{m} / z(\%) 554\left(\mathrm{M}^{+}\right), 556$. HRMS: Calculated for $\mathrm{C}_{28} \mathrm{H}_{36} \mathrm{BrF}_{4} \mathrm{Si}:$ 555.1700; Found: 555.1702.

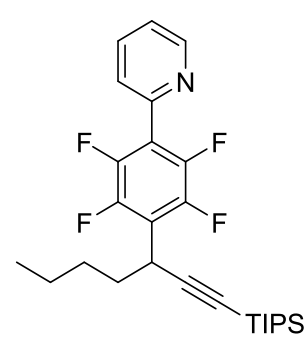

2-(2,3,5,6-Tetrafluoro-4-(1-(triisopropylsilyl)hept-1-yn-3-yl)phenyl)pyridine (4j). 3.0 equiv of fluoroarene was used. The product $(215 \mathrm{mg}, 75 \%$ yield) as a colorless oil was purified with silica gel chromatography (Petroleum ether $/ \mathrm{EtOAc}=20: 1) .{ }^{1} \mathrm{H}$ NMR $\left(500 \mathrm{MHz}, \mathrm{CDCl}_{3}\right) \delta 8.81-8.76(\mathrm{~m}, 1 \mathrm{H})$, 7.85-7.80 (m, 1H), 7.55-7.47 (m, 1H), 7.37-7.34 (m, 1H), $4.13(\mathrm{t}, J=7.8 \mathrm{~Hz}, 1 \mathrm{H}), 2.05-2.01(\mathrm{~m}, 1 \mathrm{H})$, 1.84-1.82 (m, 1H), 1.55-1.51 (m, 1H), 1.42-1.37 (m, 3H), 1.11-1.08 (m, 21H), $0.92(\mathrm{t}, J=7.0 \mathrm{~Hz}, 3 \mathrm{H})$. ${ }^{19} \mathrm{~F} \mathrm{NMR}\left(376 \mathrm{MHz}, \mathrm{CDCl}_{3}\right)-142.51(\mathrm{dd}, J=21.8,12.5 \mathrm{~Hz}, 2 \mathrm{~F}),-144.67(\mathrm{dd}, J=21.9,12.5 \mathrm{~Hz}, 2 \mathrm{~F})$. ${ }^{13} \mathrm{C} \mathrm{NMR}\left(126 \mathrm{MHz}, \mathrm{CDCl}_{3}\right) \delta 150.0,147.8,145.4(\mathrm{dm}, J=65.5 \mathrm{~Hz}), 143.5(\mathrm{dm}, J=67.3 \mathrm{~Hz}), 136.5$, 125.9, 123.5, $120.8(\mathrm{t}, J=15.7 \mathrm{~Hz}), 118.4(\mathrm{t}, J=16.2 \mathrm{~Hz}), 106.0,82.5,34.9,29.6,28.2,22.1,18.5$, 13.8, 11.2. IR (thin film) $v_{\max } 2943,2865,2172,1485,675 \mathrm{~cm}^{-1}$. MS (DART): $\mathrm{m} / z(\%) 478[\mathrm{M}+\mathrm{H}]^{+}$. HRMS: Calculated for $\mathrm{C}_{27} \mathrm{H}_{36} \mathrm{~F}_{4} \mathrm{NSi}$ : 478.2548; Found: 478.2546. 


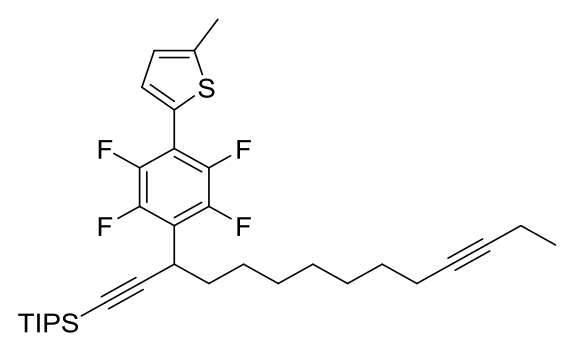

\section{Triisopropyl(3-(2,3,5,6-tetrafluoro-4-(5-methylthiophen-2-yl)phenyl)tetradeca-1,11-diyn-1-}

yl)silane (4k). 3.0 equiv of fluoroarene was used. The product ( $283 \mathrm{mg}, 80 \%$ yield) as a colorless oil was purified with silica gel chromatography (Petroleum ether / EtOAc $=100: 1) .{ }^{1} \mathrm{H}$ NMR $(400 \mathrm{MHz}$, $\left.\mathrm{CDCl}_{3}\right) \delta 7.41(\mathrm{~d}, J=3.6 \mathrm{~Hz}, 1 \mathrm{H}), 6.83(\mathrm{~d}, J=2.8 \mathrm{~Hz}, 1 \mathrm{H}), 4.07(\mathrm{t}, J=7.6 \mathrm{~Hz}, 1 \mathrm{H}), 2.55(\mathrm{~s}, 3 \mathrm{H}), 2.19$ $2.12(\mathrm{~m}, 4 \mathrm{H}), 2.05-1.95(\mathrm{~m}, 1 \mathrm{H}), 1.81-1.75(\mathrm{~m}, 1 \mathrm{H}), 1.56-1.25(\mathrm{~m}, 10 \mathrm{H}), 1.13-0.93(\mathrm{~m}, 24 \mathrm{H}) .{ }^{19} \mathrm{~F} \mathrm{NMR}$ $\left(376 \mathrm{MHz} \mathrm{CDCl}_{3}\right)-141.4(\mathrm{dd}, J=21.1,11.2 \mathrm{~Hz}, 2 \mathrm{~F}),-143.3(\mathrm{dd}, J=21.1,11.3 \mathrm{~Hz}, 2 \mathrm{~F}) .{ }^{13} \mathrm{C}$ NMR $\left(126 \mathrm{MHz}, \mathrm{CDCl}_{3}\right) \delta 144.9(\mathrm{dm}, J=240.7 \mathrm{~Hz}), 143.3(\mathrm{dm}, J=248.5 \mathrm{~Hz}), 142.9(\mathrm{t}, J=4.2 \mathrm{~Hz}), 130.2$ $(\mathrm{t}, J=6.0 \mathrm{~Hz}), 125.6,125.4(\mathrm{t}, J=2.7 \mathrm{~Hz}), 118.1(\mathrm{t}, J=16.1 \mathrm{~Hz}), 113.2(\mathrm{t}, J=15.2 \mathrm{~Hz}), 106.1,82.4$, $81.6,79.4,35.2,29.1,29.0,28.9,28.7,28.1,27.5,18.7,18.5,15.1,14.4,12.4,11.2$. IR (thin film) $v_{\max }$ 2938, 2864, 2175, $1479 \mathrm{~cm}^{-1}$. MS (DART): $\mathrm{m} / z(\%) 591[\mathrm{M}+\mathrm{H}]^{+}$. HRMS: Calculated for $\mathrm{C}_{34} \mathrm{H}_{47} \mathrm{~F}_{4} \mathrm{SSi}$ : 591.3098; Found: 591.3099.

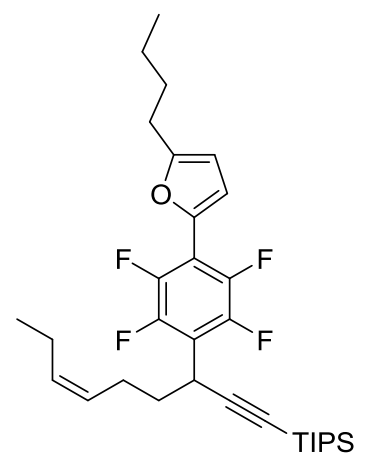

(Z)-(3-(4-(5-butylfuran-2-yl)-2,3,5,6-tetrafluorophenyl)non-6-en-1-yn-1-yl)triisopropylsilane

(41). The product (227 mg, 69\% yield) as a colorless oil was purified with silica gel chromatography $\left(\right.$ Petroleum ether / EtOAc = 50:1). ${ }^{1} \mathrm{H}$ NMR $\left(400 \mathrm{MHz}, \mathrm{CDCl}_{3}\right) \delta 6.83-6.72(\mathrm{~m}, 1 \mathrm{H}), 6.17(\mathrm{~d}, J=3.3$ $\mathrm{Hz}, 1 \mathrm{H}), 5.46(\mathrm{dt}, J=10.7,7.2 \mathrm{~Hz}, 1 \mathrm{H}), 5.32(\mathrm{dt}, J=10.7,7.2 \mathrm{~Hz}, 1 \mathrm{H}), 4.10(\mathrm{t}, J=7.4 \mathrm{~Hz}, 1 \mathrm{H}), 2.72$ $(\mathrm{t}, J=7.6 \mathrm{~Hz}, 2 \mathrm{H}), 2.32-2.20(\mathrm{~m}, 2 \mathrm{H}), 2.08-2.05(\mathrm{~m}, 3 \mathrm{H}), 1.83-1.75(\mathrm{~m}, 1 \mathrm{H}), 1.73-1.66(\mathrm{~m}, 2 \mathrm{H}), 1.47-$ $1.37(\mathrm{~m}, 2 \mathrm{H}), 1.08-1.06(\mathrm{~m}, 21 \mathrm{H}), 0.99-0.94(\mathrm{~m}, 6 \mathrm{H}) .{ }^{19} \mathrm{~F}$ NMR $\left(376 \mathrm{MHz}, \mathrm{CDCl}_{3}\right)-142.7(\mathrm{dd}, J=$ 20.8, $11.5 \mathrm{~Hz}, 2 \mathrm{~F}),-143.5(\mathrm{dd}, J=20.7,11.4 \mathrm{~Hz}, 2 \mathrm{~F}) .{ }^{13} \mathrm{C} \mathrm{NMR}\left(126 \mathrm{MHz}, \mathrm{CDCl}_{3}\right) \delta 158.4,145.0$ 
$(\mathrm{dm}, J=247.7 \mathrm{~Hz}), 142.7(\mathrm{dm}, J=249.5 \mathrm{~Hz}), 140.4,133.4,127.0,117.8(\mathrm{t}, J=16.0 \mathrm{~Hz}), 114.6(\mathrm{t}, J$ $=6.3 \mathrm{~Hz}), 110.0,107.0,106.0,82.6,35.2,30.0,27.8,27.6,25.2,22.2,20.6,18.5,14.3,13.8,11.2 . \mathrm{IR}$ (thin film) $v_{\max } 2960,2866,2175,1486,787,678 \mathrm{~cm}^{-1}$. MS (DART): $\mathrm{m} / z(\%) 549[\mathrm{M}+\mathrm{H}]^{+}$. HRMS: Calculated for $\mathrm{C}_{32} \mathrm{H}_{45} \mathrm{~F}_{4} \mathrm{OSi}$ : 549.3170; Found: 549.3169.

Unsymmetrical Synthesis of Dialkylated Fluoroarenes by Iterative C-H Functionalization of 1,2,4,5-Tetrafluorobenzene
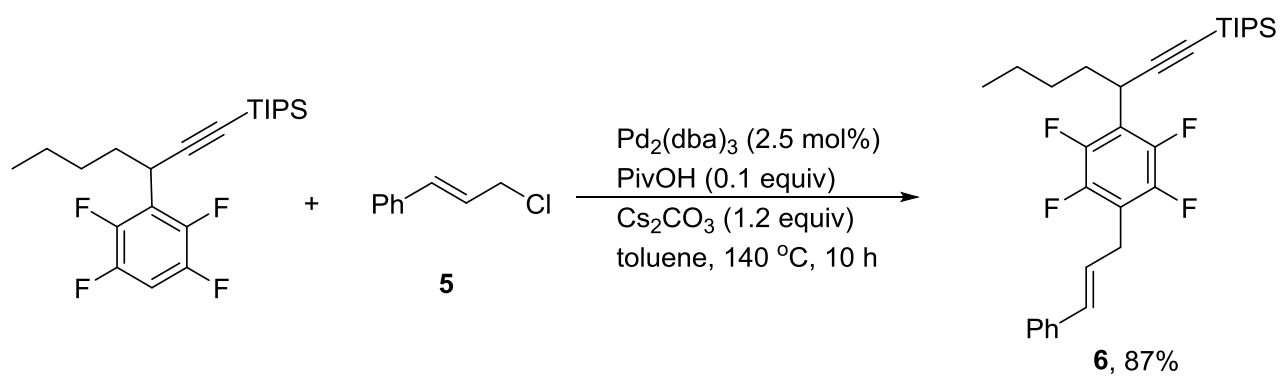

The reaction was conducted according to the literature. ${ }^{3}$ To a septum capped $25 \mathrm{~mL}$ of Schlenck tube were added $\mathrm{Pd}_{2}(\mathrm{dba})_{3}(2.5 \mathrm{~mol} \%), \mathrm{Cs}_{2} \mathrm{CO}_{3}\left(1.2\right.$ equiv) under $\mathrm{N}_{2}$, followed by toluene $2.0 \mathrm{~mL}, \mathrm{PivOH}$ (0.1 equiv), $\mathbf{4 a}$ ( $0.43 \mathrm{mmol}, 1.5$ equiv), $\mathbf{5}$ ( $0.23 \mathrm{mmol}, 1.0$ equiv), the tube was screw capped and heated to $140{ }^{\circ} \mathrm{C}$ with an oil bath. After stirring for $10 \mathrm{~h}$, the reaction mixture was cooled to room temperature, and diluted with ethyl acetate, washed with brine, dried over $\mathrm{Na}_{2} \mathrm{SO}_{4}$, filtered and concentrated. The residue was purified with silica gel chromatography (Petroleum ether (100\%)) to give compound 6 (103 mg, 87\% yield) as a yellow oil.

(3-(4-Cinnamyl-2,3,5,6-tetrafluorophenyl)hept-1-yn-1-yl)triisopropylsilane (6). ${ }^{1} \mathrm{H}$ NMR (400 $\left.\mathrm{MHz}, \mathrm{CDCl}_{3}\right) \delta 7.36-7.34(\mathrm{~m}, 2 \mathrm{H}), 7.32-7.28(\mathrm{~m}, 2 \mathrm{H}), 7.24-7.21(\mathrm{~m}, 1 \mathrm{H}), 6.52(\mathrm{~d}, J=15.7 \mathrm{~Hz}$, 1H), $6.27(\mathrm{dt}, J=15.7 \mathrm{~Hz}, 7.6 \mathrm{~Hz}, 1 \mathrm{H}), 4.08(\mathrm{t}, J=7.6 \mathrm{~Hz}, 1 \mathrm{H}), 3.62(\mathrm{~d}, J=6.4 \mathrm{~Hz}, 2 \mathrm{H}), 2.06-1.96$ (m, 1H), 1.84-1.75 (m, 1H), 1.55-1.51 (m, 1H), $1.37(\mathrm{~d}, J=6.5 \mathrm{~Hz}, 3 \mathrm{H}), 1.12-1.02(\mathrm{~m}, 21 \mathrm{H}), 0.92(\mathrm{t}$, $J=7.0 \mathrm{~Hz}, 3 \mathrm{H}) .{ }^{19} \mathrm{~F} \mathrm{NMR}\left(376 \mathrm{MHz}, \mathrm{CDCl}_{3}\right) \delta-143.3(\mathrm{dd}, J=21.6,12.5 \mathrm{~Hz}, 2 \mathrm{~F}),-145.1(\mathrm{dd}, J=$ 21.7, $12.5 \mathrm{~Hz}, 2 \mathrm{~F}) .{ }^{13} \mathrm{C} \mathrm{NMR}\left(126 \mathrm{MHz}, \mathrm{CDCl}_{3}\right) \delta 144.8(\mathrm{dm}, J=248.8 \mathrm{~Hz}), 144.5(\mathrm{dm}, J=243.4$ $\mathrm{Hz}), 136.8,132.3,128.5,127.5,126.2,124.7,118.6(\mathrm{t}, J=15.7 \mathrm{~Hz}), 116.7(\mathrm{t}, J=18.5 \mathrm{~Hz}), 106.4,82.3$,

3 Yu, Y.-B, S Fan, X Zhang. Chem. Eur. J., 2012, 18, 14643. 
35.0, 29.7, 28.1, 26.2, 22.1, 18.6, 13.9, 11.3. IR (thin film) $v_{\max } 2998,2869,2186,1513 \mathrm{~cm}^{-1}$. MS (EI): $m / z(\%) 516\left(\mathrm{M}^{+}\right), 517[\mathrm{M}+\mathrm{H}]^{+}, 473$ (100). HRMS: Calculated for $\mathrm{C}_{31} \mathrm{H}_{40} \mathrm{~F}_{4} \mathrm{Si}$ : 516.2835; Found: 516.2831.

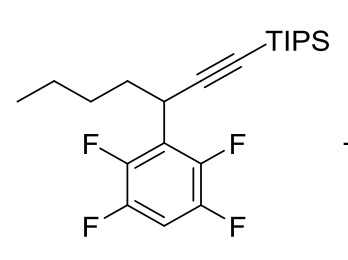

$4 \mathbf{a}$

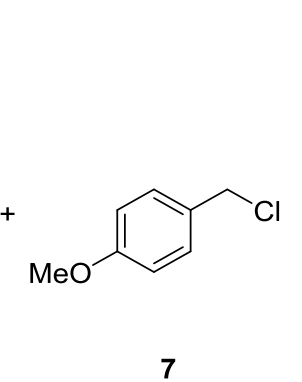

7

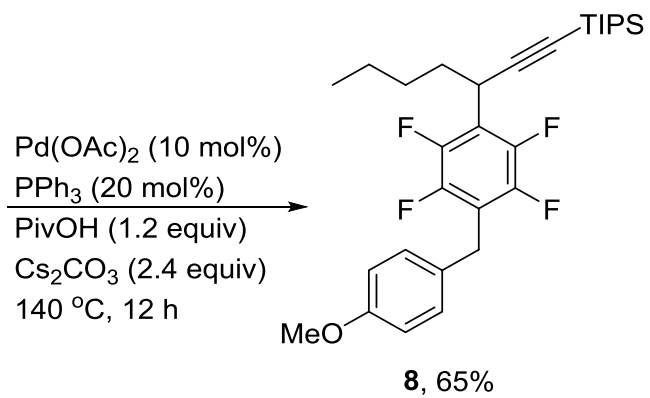

$8,65 \%$

The reaction was conducted according to the literature. ${ }^{4}$ To a septum capped $25 \mathrm{~mL}$ of Schlenck tube were added $\mathrm{Pd}(\mathrm{OAc})_{2}(10 \mathrm{~mol} \%), \mathrm{PPh}_{3}(20 \mathrm{~mol} \%), \mathrm{Cs}_{2} \mathrm{CO}_{3}\left(2.4\right.$ equiv) under $\mathrm{N}_{2}$, followed by toluene $2.0 \mathrm{~mL}, \mathrm{PivOH}$ (0.1 equiv), $4 \mathbf{a}(0.2 \mathrm{mmol}, 2.0$ equiv), and 7 ( $0.1 \mathrm{mmol}, 1.0$ equiv), the tube was screw capped and heated to $140{ }^{\circ} \mathrm{C}$ with an oil bath. After stirring for $12 \mathrm{~h}$, the reaction mixture was cooled to room temperature, and diluted with ethyl acetate, washed with brine, dried over $\mathrm{Na}_{2} \mathrm{SO}_{4}$, filtered and concentrated. The residue was purified with silica gel chromatography (Petroleum ether (100\%)) to give compound $\mathbf{8}$ (34 $\mathrm{mg}, 65 \%$ yield) as a colorless oil.

Triisopropyl(3-(2,3,5,6-tetrafluoro-4-(4-methoxybenzyl)phenyl)hept-1-yn-1-yl)silane $\quad(8) . \quad{ }^{1} \mathrm{H}$ NMR (400 MHz, $\left.\mathrm{CDCl}_{3}\right) \delta 7.21(\mathrm{~d}, J=8.4 \mathrm{~Hz}, 2 \mathrm{H}), 6.84(\mathrm{~d}, J=8.6 \mathrm{~Hz}, 2 \mathrm{H}), 4.02(\mathrm{t}, J=7.7 \mathrm{~Hz}, 1 \mathrm{H})$, $3.97(\mathrm{~s}, 2 \mathrm{H}), 3.78(\mathrm{~s}, 3 \mathrm{H}), 1.97-1.91(\mathrm{~m}, 1 \mathrm{H}), 1.77-1.69(\mathrm{~m}, 1 \mathrm{H}), 1.49-1.47(\mathrm{~m}, 1 \mathrm{H}), 1.35-1.33(\mathrm{~m}, 3 \mathrm{H})$, $1.09-1.02(\mathrm{~m}, 21 \mathrm{H}), 0.89(\mathrm{t}, J=7.0 \mathrm{~Hz}, 3 \mathrm{H}) .{ }^{19} \mathrm{~F} \mathrm{NMR}\left(376 \mathrm{MHz}, \mathrm{CDCl}_{3}\right) \delta-143.2(\mathrm{dd}, J=21.7,12.4$ $\mathrm{Hz}, 2 \mathrm{~F}),-144.8(\mathrm{dd}, J=21.8,12.5 \mathrm{~Hz}, 2 \mathrm{~F}) .{ }^{13} \mathrm{C} \mathrm{NMR}\left(126 \mathrm{MHz}, \mathrm{CDCl}_{3}\right) 158.4,144.5(\mathrm{dm}, J=244.3$ $\mathrm{Hz}), 129.9,129.6,118.5(\mathrm{t}, J=15.4 \mathrm{~Hz}), 118.2(\mathrm{t}, J=18.7 \mathrm{~Hz}), 114.1,106.4,82.2,55.2,34.9,29.6$, 28.0, 27.7, 22.1, 18.5, 13.9, 11.2. IR (thin film) $v_{\max } 2984,2963,1743,1242,1048 \mathrm{~cm}^{-1}$. MS (DART): $m / z(\%) 521[\mathrm{M}+\mathrm{H}]^{+}$. HRMS: Calculated for $\mathrm{C}_{30} \mathrm{H}_{41} \mathrm{~F}_{4} \mathrm{OSi}$ : 521.2857; Found: 521.2857.

${ }^{4}$ S Fan, C He, X Zhang. Chem. Commun., 2010, 46, 4926. 


\section{Transformations of Compound 9}

\section{Synthesis of 9}
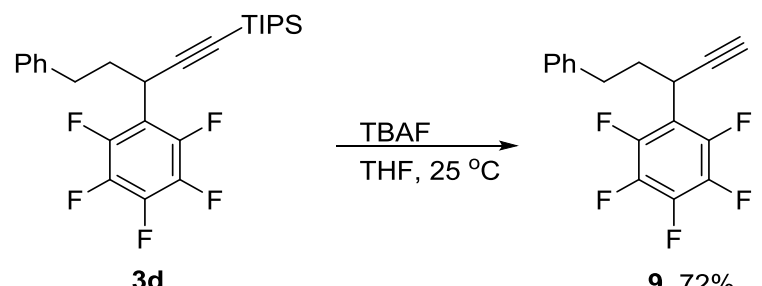

9, $72 \%$

1,2,3,4,5-Pentafluoro-6-(5-phenylpent-1-yn-3-yl)benzene (9). To a solution of HOAc (17.4 mmol, 2.9 equiv) and TBAF (1.0 M in THF, $13.2 \mathrm{~mL} 13.2 \mathrm{mmol}, 2.2$ equiv) in THF (80 mL) was added TIPS protected alkyne 3d (2.8g, $6 \mathrm{mmol}, 1.0$ equiv) at room temperature. The reaction mixture was stirred for $3 \mathrm{~h}$ and then quenched with sat. $\mathrm{NH}_{4} \mathrm{Cl}$. The reaction mixture was then diluted with water and extracted with EtOAc. The organic layers were dried over $\mathrm{NaSO}_{4}$, filtered and concentrated. The resulting crude product was purified by flash chromatography (Pentane / EtOAc $=100: 1)$ to afford terminal alkyne 9 (1.34 g, $72 \%$ yield) as a white solid (m.p. $\left.31^{\circ} \mathrm{C}\right) .{ }^{1} \mathrm{H}$ NMR $\left(400 \mathrm{MHz}, \mathrm{CDCl}_{3}\right) \delta 7.30$ - $7.25(\mathrm{~m}, 2 \mathrm{H}), 7.22$ - $7.16(\mathrm{~m}, 3 \mathrm{H}), 3.99(\mathrm{t}, J=7.3 \mathrm{~Hz}, 1 \mathrm{H}), 2.88-2.81(\mathrm{~m}, 1 \mathrm{H}), 2.73-2.65(\mathrm{~m}, 1 \mathrm{H})$, 2.12-2.03 (m, 1H), $2.24(\mathrm{~d}, J=2.5 \mathrm{~Hz}, 1 \mathrm{H}), 2.07(\mathrm{~m}, 1 \mathrm{H}) .{ }^{19} \mathrm{~F}$ NMR $\left(376 \mathrm{MHz}, \mathrm{CDCl}_{3}\right) \delta-141.8(\mathrm{dd}$, $J=21.2,7.1 \mathrm{~Hz}, 2 \mathrm{~F}),-155.8(\mathrm{t}, J=21.0 \mathrm{~Hz}, 1 \mathrm{~F}),-161.9(\mathrm{td}, J=21.6,7.6 \mathrm{~Hz}, 2 \mathrm{~F}) .{ }^{13} \mathrm{C}$ NMR $(126$ $\left.\mathrm{MHz}, \mathrm{CDCl}_{3}\right) \delta 144.9(\mathrm{dm}, J=248.9 \mathrm{~Hz}), 140.4(\mathrm{dm}, J=253.3 \mathrm{~Hz}), 140.2,137.6(\mathrm{~d}, J=253.1 \mathrm{~Hz})$, $128.5,128.3,126.3,114.6(\mathrm{td}, J=15.9,4.2 \mathrm{~Hz}), 81.7,70.6,35.9,33.6,26.2 . \mathrm{IR}\left(\mathrm{KBr}\right.$ disk) $v_{\max } 3311$, 2962, 1948, $1503 \mathrm{~cm}^{-1}$. MS (EI): $\mathrm{m} / z(\%) 310\left(\mathrm{M}^{+}\right), 311[\mathrm{M}+\mathrm{H}]^{+}, 105$ (100). HRMS: Calculated for $\mathrm{C}_{17} \mathrm{H}_{11} \mathrm{~F}_{5}: 310.0781$; Found: 310.0769.

\section{Synthesis of Compound 10a}<smiles>C#CC(CCc1ccccc1)c1c(F)c(F)c(F)c(F)c1F</smiles><smiles>CCOC(=O)OCC</smiles><smiles>C=CC(CCc1ccccc1)c1c(F)c(F)c(F)c(F)c1F</smiles>

1,2,3,4,5-Pentafluoro-6-(5-phenylpent-1-en-3-yl)benzene (10a). To a solution of 9 (62 mg, 0.2 mmol) in $10 \mathrm{~mL}$ of anhydrous EtOAc was added Lindlar catalyst $(10 \mathrm{mg})$, followed by quinoline $(10$ $\mathrm{mg}$ ). The reaction mixture was stirred for $0.5 \mathrm{~h}$ at room temperature under hydrogen atmosphere. The 
reaction mixture was filtrated through a pad of Celite ${ }^{\circledR}$ and concentrated. The resulting crude product was purified by flash chromatography (Pentane) to afford alkene 10a (53 $\mathrm{mg}, 85 \%$ yield) as a colorless oil. ${ }^{1} \mathrm{H}$ NMR (400 MHz, $\left.\mathrm{CDCl}_{3}\right) \delta$ 7.28-7.23 (m, 2H), $7.18(\mathrm{t}, J=7.3 \mathrm{~Hz}, 1 \mathrm{H}), 7.12(\mathrm{~d}, J=7.3 \mathrm{~Hz}$, 2H), 6.09-6.00 (m, 1H), 5.14-5.10 (m, 2H), $3.76(\mathrm{q}, J=7.8 \mathrm{~Hz}, 1 \mathrm{H}), 2.68-2.61(\mathrm{~m}, 1 \mathrm{H}), 2.55-2.48$ (m, 1H), 2.17-2.10 (m, 2H). ${ }^{19} \mathrm{~F}$ NMR (376 MHz, $\left.\mathrm{CDCl}_{3}\right)-143.0(\mathrm{dd}, J=21.3,6.7 \mathrm{~Hz}, 2 \mathrm{~F}),-159.2(\mathrm{t}$, $J=20.3 \mathrm{~Hz}, 1 \mathrm{~F}),-164.1(\mathrm{dt}, J=20.9,7.5 \mathrm{~Hz}, 2 \mathrm{~F}) .{ }^{13} \mathrm{C} \mathrm{NMR}\left(126 \mathrm{MHz}, \mathrm{CDCl}_{3}\right) \delta 144.9(\mathrm{dm}, J=246.2$ $\mathrm{Hz}), 141.0,139.7(\mathrm{dm}, J=257.9 \mathrm{~Hz}), 137.6(\mathrm{dm}, J=257.7 \mathrm{~Hz}), 137.5,128.4,128.3,126.1,116.9$, $110.0(\mathrm{~m}), 39.7,34.7,33.9$. IR (thin film) $v_{\max } 3086,2932,1499,975 \mathrm{~cm}^{-1}$. MS (EI): $\mathrm{m} / z(\%) 312\left(\mathrm{M}^{+}\right)$, $313[\mathrm{M}+\mathrm{H}]^{+}, 105$ (100). HRMS: Calculated for $\mathrm{C}_{17} \mathrm{H}_{13} \mathrm{~F}_{5}$ : 312.0937 ; Found: 312.0942.

\section{Synthesis of Compound 10b}
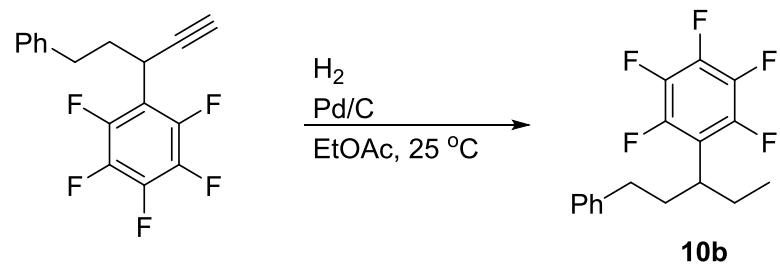

1,2,3,4,5-Pentafluoro-6-(1-phenylpentan-3-yl)benzene (10b). To a solution of 9 (62 mg, $0.2 \mathrm{mmol})$ in $10 \mathrm{~mL}$ of anhydrous EtOAc was added $\mathrm{Pd} / \mathrm{C}(10 \mathrm{mg})$. The reaction mixture was stirred for $4 \mathrm{~h}$ at room temperature under hydrogen atmosphere. The reaction mixture was filtrated through a pad of Celite ${ }^{\circledR}$ and concentrated. The resulting crude product was purified by flash chromatography (Pentane) to afford alkane $\mathbf{1 0 b}\left(59 \mathrm{mg}, 95 \%\right.$ yield) as a colorless oil. ${ }^{1} \mathrm{H} \mathrm{NMR}\left(400 \mathrm{MHz}, \mathrm{CDCl}_{3}\right) \delta 7.24(\mathrm{dd}, J$ = 7.6, 7.1 Hz, 2H), $7.15(\mathrm{dd}, J=8.4,6.2 \mathrm{~Hz}, 1 \mathrm{H}), 7.08(\mathrm{~d}, J=6.9 \mathrm{~Hz}, 2 \mathrm{H}), 3.01(\mathrm{tt}, J=9.4,5.9 \mathrm{~Hz}$, 1H), 2.56-2.42 (m, 2H), 2.11-1.99 (m, 2H), 1.79-1.70 (m, 2H), $0.80(\mathrm{t}, J=7.4 \mathrm{~Hz}, 3 \mathrm{H}) .{ }^{19} \mathrm{~F} \mathrm{NMR}(376$ $\left.\mathrm{MHz}, \mathrm{CDCl}_{3}\right)-142.1(\mathrm{dd}, J=22.5,7.9 \mathrm{~Hz}, 2 \mathrm{~F}),-157.6(\mathrm{t}, J=21.0 \mathrm{~Hz}, 1 \mathrm{~F}),-162.8(\mathrm{td}, J=22.7,8.2$ $\mathrm{Hz}, 2 \mathrm{~F}) .{ }^{13} \mathrm{C}$ NMR $\left(126 \mathrm{MHz}, \mathrm{CDCl}_{3}\right) \delta 145.4(\mathrm{dm}, J=245.7 \mathrm{~Hz}), 141.4,139.4(\mathrm{dm}, J=238.3 \mathrm{~Hz})$, $137.4(\mathrm{dm}, J=237.9 \mathrm{~Hz}), 128.3,128.2,125.9,117.6(\mathrm{tm}, J=16.8 \mathrm{~Hz}), 38.0,35.1,34.3,27.0,12.3$. IR (thin film) $v_{\max }$ 3087, 2933, 1499, $973 \mathrm{~cm}^{-1}$. MS (EI): $\mathrm{m} / z$ (\%) $314\left(\mathrm{M}^{+}\right), 181,91$ (100). HRMS: Calculated for $\mathrm{C}_{17} \mathrm{H}_{15} \mathrm{~F}_{5}$ : 314.1094; Found: 314.1091. 


\section{Synthesis of Compound $10 c^{5}$}
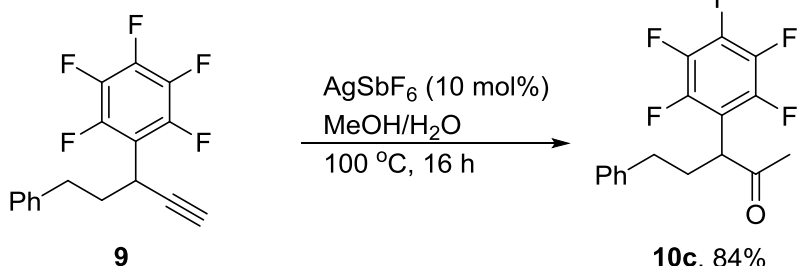

$10 c, 84 \%$

3-(Perfluorophenyl)-5-phenylpentan-2-one (10c). To a septum capped $25 \mathrm{~mL}$ of Schlenck tube were added $\mathrm{AgSbF}_{6}(0.02 \mathrm{mmol}, 10 \mathrm{~mol} \%), 9(62 \mathrm{mg}, 0.2 \mathrm{mmol}), \mathrm{MeOH}(0.3 \mathrm{~mL})$ and $\mathrm{H}_{2} \mathrm{O}(30 \mu \mathrm{L})$, the tube was screw capped and then heated to $100{ }^{\circ} \mathrm{C}$ with an oil bath. After stirring for $16 \mathrm{~h}$, the reaction mixture was cooled to room temperature, and diluted with ethyl acetate, washed with brine, dried over $\mathrm{Na}_{2} \mathrm{SO}_{4}$, filtered and concentrated. The residue was purified with silica gel chromatography (Petroleum ether / EtOAc 10:1) to product $10 \mathrm{c}\left(55 \mathrm{mg}, 84 \%\right.$ yield) as a colorless oil. ${ }^{1} \mathrm{H} \mathrm{NMR}\left(400 \mathrm{MHz}, \mathrm{CDCl}_{3}\right)$ $\delta$ 7.28-7.25 (m, 2H), 7.20-7.16 (m, 1H), 7.12-7.10 (m, 2H), $3.90(\mathrm{dd}, J=9.5,4.4 \mathrm{~Hz}, 1 \mathrm{H}), 2.58-2.53$ (m, 3H), 2.18-2.10 (m, 4H). ${ }^{19} \mathrm{~F}$ NMR (376 MHz, $\left.\mathrm{CDCl}_{3}\right)-141.0(\mathrm{dd}, J=21.2,6.8 \mathrm{~Hz}, 2 \mathrm{~F}),-154.7$ (t, $J=20.9 \mathrm{~Hz}, 1 \mathrm{~F}),-161.4(\mathrm{qd}, J=9.2,2.4 \mathrm{~Hz}, 2 \mathrm{~F}) .{ }^{13} \mathrm{C}$ NMR $\left(126 \mathrm{MHz}, \mathrm{CDCl}_{3}\right) \delta 203.2,145.2(\mathrm{dm}, J$ $=250.4 \mathrm{~Hz}), 140.5(\mathrm{dm}, J=254.5 \mathrm{~Hz}), 140.3,137.7(\mathrm{dm}, J=253.4 \mathrm{~Hz}), 128.5,128.3,126.3,112.7$ (td, $J=16.6 \mathrm{~Hz}, 4.0 \mathrm{~Hz}), 48.3,33.7,30.3,28.1$. IR (thin film) $v_{\max } 3029,2929,1727,1501,968 \mathrm{~cm}^{-1}$. MS (EI): $m / z(\%) 328\left(\mathrm{M}^{+}\right), 329[\mathrm{M}+\mathrm{H}]^{+}, 43$ (100). HRMS: Calculated for $\mathrm{C}_{17} \mathrm{H}_{13} \mathrm{~F}_{5} \mathrm{O}$ : 328.0887; Found: 328.0881.

\section{Synthesis of Compound 10d}
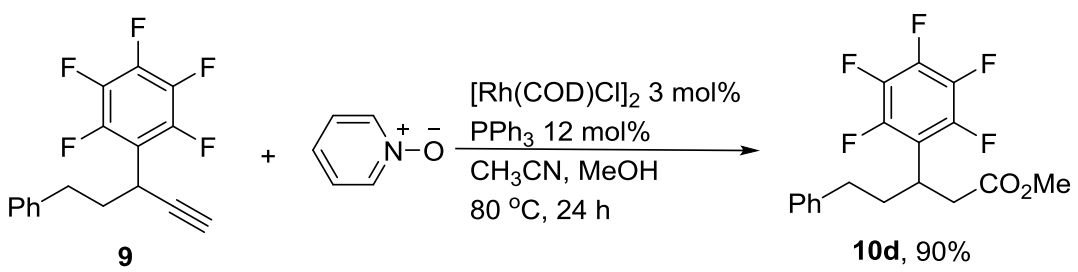

Methyl 3-(perfluorophenyl)-5-phenylpentanoate (10d). To a septum capped $25 \mathrm{~mL}$ of Schlenck tube were added $[\mathrm{Rh}(\mathrm{COD}) \mathrm{Cl}]_{2}(3 \mathrm{mg}, 0.006 \mathrm{mmol}, 3 \mathrm{~mol} \%), \mathrm{PPh}_{3}(0.024 \mathrm{mmol}, 12 \mathrm{~mol} \%)$, Pyridine $N$ oxide ( 0.24 mmol, 1.2 mol equiv), 9 ( $0.2 \mathrm{mmol}, 1.0$ equiv), $\mathrm{MeOH}$ (1.0 mmol, 5.0 equiv), and $\mathrm{CH}_{3} \mathrm{CN}$

\footnotetext{
5 Thuong, M. B. T.; Mann, A.; Wagner, A. Chem. Commun. 2012, 48, 434-436.

${ }^{6}$ Kim, I.; Lee, C. Angew. Chem., Int. Ed. 2013, 52, 10023.
} 
$(1.5 \mathrm{~mL})$. The tube was screw capped and then heated to $80{ }^{\circ} \mathrm{C}$ with an oil bath and. After stirring for $24 \mathrm{~h}$, the reaction mixture was cooled to room temperature, and diluted with ethyl acetate, washed with brine, dried over $\mathrm{Na}_{2} \mathrm{SO}_{4}$, filtered and concentrated. The residue was purified with silica gel chromatography (Petroleum ether / EtOAc 10:1) to give product 10d (65 mg, 90\% yield) as a colorless oil. ${ }^{1} \mathrm{H}$ NMR (400 MHz, $\left.\mathrm{CDCl}_{3}\right) \delta 7.26(\mathrm{t}, J=7.4 \mathrm{~Hz}, 2 \mathrm{H}), 7.18(\mathrm{~d}, J=7.2 \mathrm{~Hz}, 1 \mathrm{H}), 7.10(\mathrm{~d}, J=7.2$ $\mathrm{Hz}, 2 \mathrm{H}), 3.68-3.60(\mathrm{~m}, 4 \mathrm{H}), 2.79$ (d, $J=8.1 \mathrm{~Hz}, 2 \mathrm{H}), 2.62-2.54(\mathrm{~m}, 1 \mathrm{H}), 2.50-2.43(\mathrm{~m}, 1 \mathrm{H}), 2.16-1.99$ (m, 2H). ${ }^{19} \mathrm{~F}$ NMR $\left(376 \mathrm{MHz}, \mathrm{CDCl}_{3}\right)-141.8(\mathrm{dd}, J=22.0,7.1 \mathrm{~Hz}, 2 \mathrm{~F}),-156.4(\mathrm{t}, J=21.0 \mathrm{~Hz}, 1 \mathrm{~F}),-$ $162.2(\mathrm{dt}, J=21.9,7.5 \mathrm{~Hz}, 2 \mathrm{~F}) .{ }^{13} \mathrm{C} \mathrm{NMR}\left(126 \mathrm{MHz}, \mathrm{CDCl}_{3}\right) \delta 171.8,145.3(\mathrm{dm}, J=246.8 \mathrm{~Hz}), 140.6$, $139.9(\mathrm{dm}, J=238.7 \mathrm{~Hz}), 137.5(\mathrm{dm}, J=250.9 \mathrm{~Hz}), 128.4,128.1,126.1,116.3(\mathrm{tm}, J=16.2 \mathrm{~Hz}), 51.8$, 38.2, 35.0, 33.9, 32.1. IR (thin film) $v_{\max }$ 3064, 2954, 1742, 1298, $1199 \mathrm{~cm}^{-1}$. MS (EI): $\mathrm{m} / z$ (\%) 358 $\left(\mathrm{M}^{+}\right), 359[\mathrm{M}+\mathrm{H}]^{+}, 91$ (100). HRMS: Calculated for $\mathrm{C}_{18} \mathrm{H}_{15} \mathrm{~F}_{5} \mathrm{O}_{2}$ : 358.0992; Found: 358.0994 .

\section{Synthesis of Compound 10e}
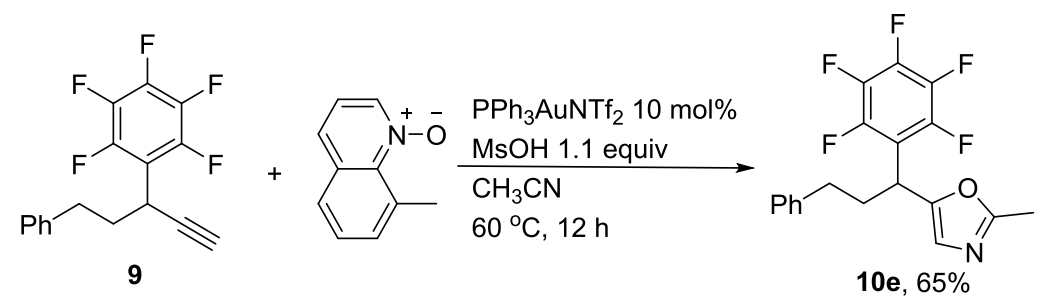

2-Methyl-5-(1-(perfluorophenyl)-3-phenylpropyl)oxazole (10e). To a septum capped $25 \mathrm{~mL}$ of Schlenck tube were added $\mathrm{PPh}_{3} \mathrm{AuNTf}_{2}(0.02 \mathrm{mmol}, 10 \mathrm{~mol} \%), 9$ (0.2 mmol, 1.0 equiv), 8methylquinoline 1-oxide (0.26 mmol, 1.3 equiv) under $\mathrm{N}_{2}$, followed by $\mathrm{CH}_{3} \mathrm{CN}(1.5 \mathrm{~mL})$ and $\mathrm{MsOH}$ (0.22 mmol, 1.1 equiv), the tube was screw capped and then heated to $60{ }^{\circ} \mathrm{C}$ with an oil bath. After stirring for $12 \mathrm{~h}$, the reaction mixture was cooled to room temperature, and diluted with ethyl acetate, washed with brine, dried over $\mathrm{Na}_{2} \mathrm{SO}_{4}$, filtered and concentrated. The residue was purified with silica gel chromatography (Petroleum ether / EtOAc 5:1) to give product 10e (48 mg, 65\% yield) as a white solid (m.p. $\left.68{ }^{\circ} \mathrm{C}\right) .{ }^{1} \mathrm{H}$ NMR $\left(400 \mathrm{MHz}, \mathrm{CDCl}_{3}\right) \delta 7.26(\mathrm{t}, J=7.4 \mathrm{~Hz}, 2 \mathrm{H}), 7.18(\mathrm{t}, J=7.3 \mathrm{~Hz}, 1 \mathrm{H})$, $7.11(\mathrm{~d}, J=7.2 \mathrm{~Hz}, 2 \mathrm{H}), 6.74(\mathrm{~s}, 1 \mathrm{H}), 4.39(\mathrm{t}, J=7.8 \mathrm{~Hz}, 1 \mathrm{H}), 2.67-2.56(\mathrm{~m}, 2 \mathrm{H}), 2.54-2.43(\mathrm{~m}, 1 \mathrm{H})$, 2.41-2.37 (m, 4H). ${ }^{19} \mathrm{~F}$ NMR (376 MHz, $\left.\mathrm{CDCl}_{3}\right) \delta-141.8(\mathrm{dd}, J=21.8,7.4 \mathrm{~Hz}, 2 \mathrm{~F}),-155.4(\mathrm{t}, J=21.0$

${ }^{7}$ He, W.; Li, C.; Zhang, L. J. Am. Chem. Soc. 2011, 133, 8482. 
$\mathrm{Hz}, 1 \mathrm{~F}),-161.7(\mathrm{td}, J=21.8,7.6 \mathrm{~Hz}, 2 \mathrm{~F}) .{ }^{13} \mathrm{C} \mathrm{NMR}\left(126 \mathrm{MHz}, \mathrm{CDCl}_{3}\right) \delta 161.0,150.5,145.2(\mathrm{dm}, J$ $=248.9 \mathrm{~Hz}), 140.3(\mathrm{dm}, J=253.9 \mathrm{~Hz}), 140.2,137.6(\mathrm{dm}, J=244.1 \mathrm{~Hz}), 128.5,128.2,126.3,123.1$, $114.2(\mathrm{tm}, J=16.8 \mathrm{~Hz}), 33.7,32.5,32.2,13.93$. IR (KBr disk) $v_{\max } 3028,2933,1739,1502,701 \mathrm{~cm}^{-1}$. MS (EI): m/z (\%) $367\left(\mathrm{M}^{+}\right), 368[\mathrm{M}+\mathrm{H}]^{+}, 105$ (100). HRMS: Calculated for $\mathrm{C}_{19} \mathrm{H}_{14} \mathrm{NOF}_{5}: 367.0996$; Found: 367.0992.

\section{Synthesis of Compound $10 \mathrm{f}^{8}$}

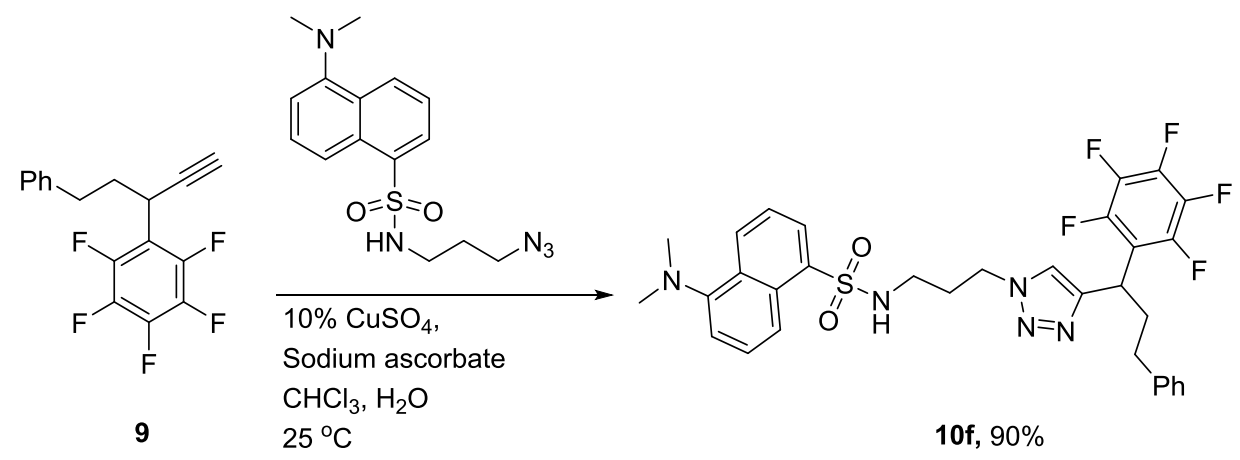

\section{5-(Dimethylamino)-N-(3-(4-(1-(perfluorophenyl)-3-phenylpropyl)-1H-1,2,3-triazol-1-}

yl)propyl)naphthalene-1-sulfonamide (10f). To a solution of 9 ( $0.43 \mathrm{mmol}, 1.0$ equiv) in $3.0 \mathrm{~mL}$ of $\mathrm{CHCl}_{3}$ and $\mathrm{H}_{2} \mathrm{O}(0.2 \mathrm{~mL})$ were added Dans- $\left(\mathrm{CH}_{2}\right)_{3}-\mathrm{N}_{3}\left(0.43 \mathrm{mmol}, 1.0\right.$ equiv), $\mathrm{CuSO}_{4}(0.043 \mathrm{mmol}$, 0.1 equiv), and sodium ascorbate $(0.086 \mathrm{mmol}, 0.2$ equiv $)$. After stirring for $24 \mathrm{~h}$ at room temperature, the mixture was quenched by $\mathrm{NH}_{4} \mathrm{Cl}$ (sat.), and diluted with ethyl acetate, washed with brine, dried over $\mathrm{Na}_{2} \mathrm{SO}_{4}$, filtered and concentrated. The residue was purified with silica gel chromatography (Petroleum ether / EtOAc 2:1 to 1:2) to give product 10f (249 mg, 90\% yield) as a yellow solid (m.p. $\left.59{ }^{\circ} \mathrm{C}\right) .{ }^{1} \mathrm{H}$ NMR $\left(400 \mathrm{MHz}, \mathrm{CDCl}_{3}\right) \delta 8.49(\mathrm{~d}, J=8.5 \mathrm{~Hz}, 1 \mathrm{H}), 8.28(\mathrm{~d}, J=8.6 \mathrm{~Hz}, 1 \mathrm{H}), 8.16(\mathrm{~d}, J=$ $7.2 \mathrm{~Hz}, 1 \mathrm{H}), 7.47-7.42(\mathrm{~m}, 3 \mathrm{H}), 7.24-7.20(\mathrm{~m}, 2 \mathrm{H}), 7.16-7.09(\mathrm{~m}, 4 \mathrm{H}), 6.05(\mathrm{t}, J=6.2 \mathrm{~Hz}, 1 \mathrm{H})$, $4.56(\mathrm{dd}, J=9.1,6.3 \mathrm{~Hz}, 1 \mathrm{H}), 4.30(\mathrm{t}, J=6.5 \mathrm{~Hz}, 2 \mathrm{H}), 2.83(\mathrm{~s}, 8 \mathrm{H}), 2.69-2.31(\mathrm{~m}, 4 \mathrm{H}), 1.99-1.96$ $(\mathrm{m}, 2 \mathrm{H}) .{ }^{19} \mathrm{~F}$ NMR $\left(376 \mathrm{MHz}, \mathrm{CDCl}_{3}\right) \delta-141.5(\mathrm{dd}, J=22.3,7.0 \mathrm{~Hz}, 2 \mathrm{~F}),-156.3(\mathrm{t}, J=21.1 \mathrm{~Hz}, 1 \mathrm{~F})$, $-162.0(\mathrm{td}, J=22.1,7.4 \mathrm{~Hz}, 2 \mathrm{~F}) .{ }^{13} \mathrm{C} \mathrm{NMR}\left(126 \mathrm{MHz}, \mathrm{CDCl}_{3}\right) \delta 152.1,147.8,145.2(\mathrm{dm}, J=249.8$ $\mathrm{Hz}), 140.6,140.1(\mathrm{dm}, J=245.7 \mathrm{~Hz}), 137.6(\mathrm{dm}, J=250.1 \mathrm{~Hz}), 134.3,130.7,129.9,129.6,129.5$, 128.6, 128.5, 128.3, 126.2, 123.2, 121.8, 118.4, 115.9 (tm, $J=16.3 \mathrm{~Hz}), 115.3,47.0,45.3,39.8,34.1$, 34.0, 32.6, 30.2. IR (KBr disk) $v_{\max } 3289,3140,2929,1654,1575,1522,1500 \mathrm{~cm}^{-1}$. MS (MALDI):

${ }^{8}$ Lewis, W. G.; Magallon, F. G.; Fokin, V. V.; Finn, M. G. J. Am. Chem. Soc. 2004, 126, 9152. 
$\left.m / z(\%) 643\left(\mathrm{M}^{+}\right), 644\left[(\mathrm{M}+\mathrm{H})^{+}, 100\right)\right]$. HRMS: Calculated for $\mathrm{C}_{32} \mathrm{H}_{30} \mathrm{~F}_{5} \mathrm{~N}_{5} \mathrm{O}_{2} \mathrm{~S}$ : 644.2113; Found: 644.2103. Anal. Calcd. For $\mathrm{C}_{32} \mathrm{H}_{30} \mathrm{~F}_{5} \mathrm{~N}_{5} \mathrm{O}_{2} \mathrm{~S}$ : C, 59.71; H, 4.70; Found: C, 59.47; H, 4.72.

\section{Mechanistic studies}

\section{Synthesis of Optically Pure Propargyl Alcohol ${ }^{9}$}

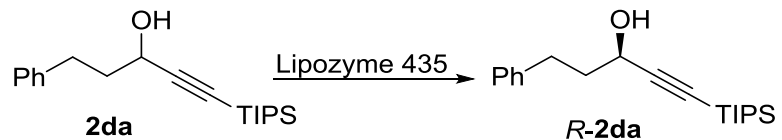

$(\boldsymbol{R})-5$-phenyl-1-(triisopropylsilyl)pent-1-yn-3-ol $\quad(\boldsymbol{R}$-2da). $\quad$ To a solution $\quad$ of 5-phenyl-1(triisopropylsilyl)pent-1-yn-3-ol (2da) $1.93 \mathrm{~g}$ (6.1 mmol, 1.0 equiv) in vinyl acetate (12 mL) was added Lipozyme $435(250 \mathrm{mg})$. The reaction mixture was heated to $80{ }^{\circ} \mathrm{C}$ and stirred for $24 \mathrm{~h}$. The reaction was then cooled to room temperature and concentrated. The residue was purified with silica gel chromatography (Petroleum ether / EtOAc 30:1) to give product $R$-2da $(0.81 \mathrm{~g}, 42 \%$ yield) as a colorless oil. ${ }^{1} \mathrm{H}$ NMR $\left(400 \mathrm{MHz}, \mathrm{CDCl}_{3}\right) \delta$ 7.32-7.28 (m, 2H), 7.26-7.16 (m, 3H), $5.42(\mathrm{t}, J=6.5 \mathrm{~Hz}$, $1 \mathrm{H}), 4.41(\mathrm{t}, J=6.5 \mathrm{~Hz}, 1 \mathrm{H}), 2.86-2.80(\mathrm{~m}, 2 \mathrm{H}), 2.16-1.95(\mathrm{~m}, 2 \mathrm{H}), 1.24-0.88(\mathrm{~m}, 21 \mathrm{H}) .{ }^{13} \mathrm{C} \mathrm{NMR}$ $\left(126 \mathrm{MHz}, \mathrm{CDCl}_{3}\right) \delta 141.5,128.5,128.5,126.0,108.6,85.9,62.3,39.7,31.6,18.7,11.2 . \mathrm{IR}$ (thin film) $v_{\max } 3355,2943,2865,2169,1463 \mathrm{~cm}^{-1}$. MS (EI): $\mathrm{m} / z(\%) 314[\mathrm{M}-2 \mathrm{H}]^{+}, 273,255,91(100) . \mathrm{HRMS}:$ Calculated for $\mathrm{C}_{20} \mathrm{H}_{30} \mathrm{OSi}$ : 314.2069; Found: 314.2074. $[\alpha]^{26}{ }_{\mathrm{D}}=+26.8\left(c=0.95, \mathrm{CHCl}_{3}\right)$ for a $99 \%$ ee sample. Enantiomeric purity was determined by chiral HPLC analysis (Cellulose-1, flow $0.7 \mathrm{~mL} / \mathrm{min}$, $\mathrm{MeOH} / \mathrm{H}_{2} \mathrm{O}=90: 10,214 \mathrm{~nm}, \mathrm{t}_{\mathrm{R}}=11.406 \mathrm{~min}$ (major) and $\mathrm{t}_{\mathrm{R}}=13.022$ (minor).

\section{Characterization of Absolute Configuration of Propargyl Alcohol $R$-2da by Mosher's Method ${ }^{10}$}

To a stirred solution of $\boldsymbol{R}-\mathbf{2 d a}(42 \mathrm{mg}, 0.13 \mathrm{mmol})$ in $\mathrm{CH}_{2} \mathrm{Cl}_{2}(3.0 \mathrm{~mL})$ was added $(S)-(-)-\mathrm{MTPA}-\mathrm{Cl}$ (0.2 mmol, 1.5 equiv), DMAP (0.01 mmol, 0.05 equiv) and $\mathrm{NEt}_{3}(0.4 \mathrm{mmol}, 2.0$ equiv) at room temperature. After the reaction mixture was stirred for $16 \mathrm{~h}$, the solvent was removed. The residue was purified by flash chromatography $($ EtOAc/pentane $=10: 100)$ to afford the $(S)$-MTPA ester.

Data for the (S)-MTPA ester of $\boldsymbol{R}$-2da: ${ }^{1} \mathrm{H}$ NMR (400 MHz, CDCl3) $\delta 7.59-7.51$ (m, 2H), 7.44$7.35(\mathrm{~m}, 3 \mathrm{H}), 7.31-7.29(\mathrm{~m}, 2 \mathrm{H}), 7.22(\mathrm{dd}, J=7.7,6.5 \mathrm{~Hz}, 1 \mathrm{H}), 7.18(\mathrm{dd}, J=8.9,4.3 \mathrm{~Hz}, 2 \mathrm{H}), 5.55$

\footnotetext{
9 X. Zhang; Z. Lu; C. Fu; S. Ma. Org. Biomol. Chem. 2009, 7, 3258.

10 Dale, J. A.; Mosher, H. S. J. Am. Chem. Soc. 1973, 95, 512.
} 
(t, $J=6.6 \mathrm{~Hz}, 1 \mathrm{H}), 3.55(\mathrm{~s}, 3 \mathrm{H}), 2.83-2.78(\mathrm{~m}, 2 \mathrm{H}), 2.27-2.07(\mathrm{~m}, 2 \mathrm{H}), 1.07-1.05(\mathrm{~m}, 21 \mathrm{H})$.

Similarly, the $(\boldsymbol{R})$-MTPA ester was prepared with $(R)-(+)$-MTPA-Cl.

Data for the (R)-MTPA ester of $\boldsymbol{R}$-2da: ${ }^{1} \mathrm{H}$ NMR $\left(400 \mathrm{MHz}, \mathrm{CDCl}_{3}\right) \delta 7.63-7.55(\mathrm{~m}, 2 \mathrm{H}), 7.45-$ $7.35(\mathrm{~m}, 3 \mathrm{H}), 7.29-7.25(\mathrm{~m}, 2 \mathrm{H}), 7.19(\mathrm{t}, J=7.3 \mathrm{~Hz}, 1 \mathrm{H}), 7.10(\mathrm{~d}, J=6.9 \mathrm{~Hz}, 2 \mathrm{H}), 5.57(\mathrm{t}, J=6.5$ $\mathrm{Hz}, 1 \mathrm{H}), 3.60$ (s, 3H), 2.82-2.59 (m, 2H), 2.21-2.01 (m, 2H), 1.09-1.07 (m, 21H).

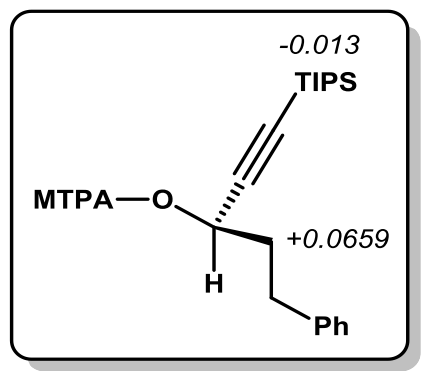

A $\Delta \delta$ ppm $\left(\Delta \delta_{\mathrm{S}}-\Delta \delta_{R}\right)$ was observed by comparison of ${ }^{1} \mathrm{H}$-NMR resonances of $S$ - and $R$ - Mosher ester derivatives. On the basis of the Mosher's conformational model, the configuration of 2 da should be $R$, which is consistent with the configuration derived from above enzymatic resolution.

\section{Synthesis of $R-2 d^{1}$}

(R)-Diethyl (5-phenyl-1-(triisopropylsilyl)pent-1-yn-3-yl) phosphate ( $\boldsymbol{R}$-3d). To a flask were added $\boldsymbol{R}$-2da (0.81 g, $4.0 \mathrm{mmol}, 1.0$ equiv), 4-dimethylaminopyridine ( $0.4 \mathrm{mmol}, 0.1$ equiv), anhydrous $\mathrm{CH}_{2} \mathrm{Cl}_{2}$, and diethylchlorophosphate (5.2 mmol, 1.3 equiv). The resulting mixture was then cooled to $0{ }^{\circ} \mathrm{C}$ and triethylamine ( $4.8 \mathrm{mmol}, 1.2$ equiv) was added. The reaction mixture was allowed to warm to room temperature with stirring. After consumption of the alcohol (TLC monitoring), the mixture was quenched with saturated aq. $\mathrm{NH}_{4} \mathrm{Cl}$ and extracted with ethyl acetate three times. The combined organic layers were washed with brine, dried over $\mathrm{Na}_{2} \mathrm{SO}_{4}$, and concentrated. The residue was purified with silica gel chromatography (Petroleum ether / EtOAc 3:1) to give product $R-\mathbf{2 d}(1.35 \mathrm{~g}$, $75 \%$ yield) as a colorless oil. $R-\mathbf{2 d}:[\alpha]^{26} \mathrm{D}=-8.9\left(c=1.2, \mathrm{CHCl}_{3}\right)$ for a $99 \%$ ee sample. Enantiomeric purity was determined by chiral HPLC analysis (ID3, flow $0.7 \mathrm{~mL} / \mathrm{min}$, Hexane: $i-\mathrm{PrOH}=98: 2,214 \mathrm{~nm}, \mathrm{t}_{\mathrm{R}}=$ 11.48 min (major) and $t_{R}=13.35$ (minor). 


\section{Synthesis of Complex $\mathrm{C}_{6} \mathrm{~F}_{5} \mathrm{H} \cdot \mathrm{dioxane}^{11}$}

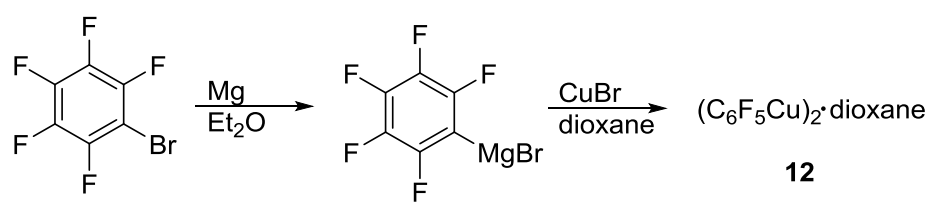

To a three-necked, round-bottomed flask fitted with a condenser bearing a nitrogen inlet were charged with magnesium turnings (1.1 g, $46 \mathrm{mmol}, 1.0$ equiv), $\mathrm{Et}_{2} \mathrm{O} 37 \mathrm{~mL}$, and a few iodine seed. Then 1bromo-2,3,4,5,6-pentafluorobenzene ( $46 \mathrm{mmol}, 1.0$ equiv) was added dropwise at a rate that maintains a gentle reflux. The reflux is maintained for another $1 \mathrm{~h}$ by heating at $35^{\circ}$. The resulting black solution is cooled to room temperature, and $92 \mathrm{mmol}$ (2.0 equiv) of powdered and anhydrous copper(I) bromide (92 mmol, 2.0 equiv) was added in three intervals. The brown mixture is stirred for 30 minutes, $20 \mathrm{~mL}$ of $\mathrm{Et}_{2} \mathrm{O}$ is added, and the mixture heated at reflux for another 30 minutes. After the mixture was cooled to room temperature, $\mathrm{Et}_{2} \mathrm{O}(80 \mathrm{~mL})$ and dioxane $(20 \mathrm{~mL})$ was added carefully and stirred for another $0.5 \mathrm{~h}$ at room temperature. The supernatant was transferred to a Schlenck flask by double-point needles with an attached nitrogen atmosphere. The solution was evaporated to dryness under reduced pressure with an oil bath at $40^{\circ}$, and the pale-yellow solid 12 (2.74 g, 22\% yield) was obtained. Compound 12 is known. ${ }^{11}{ }^{1} \mathrm{H}$ NMR $\left(400 \mathrm{MHz}, \mathrm{CDCl}_{3}\right) \delta 3.67$ (s, 8H), ${ }^{19} \mathrm{~F}$ NMR $\left(376 \mathrm{MHz}, \mathrm{CDCl}_{3}\right)-102.9(\mathrm{~m}, 2 \mathrm{~F})$, $-140.4(\mathrm{t}, J=20 \mathrm{~Hz}, 1 \mathrm{~F}),-156.8(\mathrm{~m}, 2 \mathrm{~F})$.

\section{Scheme S1. Scheme Reaction of Complex $\left(\mathrm{C}_{6} \mathrm{~F}_{5} \mathrm{Cu}\right)_{2} \cdot \operatorname{dioxane} 12$ with $R$-2d}

a)<smiles>CCOC(C#C[In])CCc1ccccc1</smiles>

$R-\mathbf{2 d},(99 \%$ ee $)$

b)

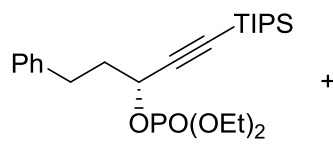

$R-2 d,(99 \%$ ee $)$

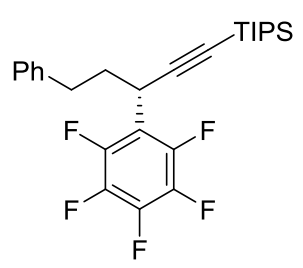

S-3d $(98 \%$ ee $), 45 \%$ yield

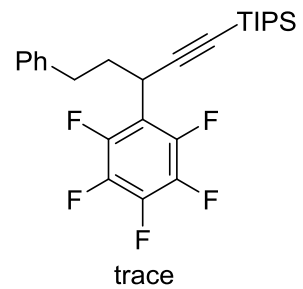

11 Cairncross, A.; Sheppard, W. A.; Wonchoba, E.; Guildford, W. J.; House, C. B.; Coates, R. M. Org. Synth. 1980, 59, 122. 
To a septum capped $25 \mathrm{~mL}$ of Schlenck tube were added $\left(\mathrm{C}_{6} \mathrm{~F}_{5} \mathrm{Cu}\right)_{2} \cdot$ dioxane $(164 \mathrm{mg}, 0.3 \mathrm{mmol}, 0.5$ equiv), under $\mathrm{N}_{2}$, followed by THF $(2.0 \mathrm{~mL})$ and $\boldsymbol{R}-\mathbf{2 d}(0.6 \mathrm{mmol}, 2.0$ equiv), the tube was screw capped and the reaction was stirred at room temperature or at $80{ }^{\circ} \mathrm{C}$. After stirring for $12 \mathrm{~h}$, the reaction mixture was cooled to room temperature and the yield was determined by ${ }^{19} \mathrm{~F}$ NMR using fluorobenzene as an internal standard. If necessary, the mixture was diluted with ethyl acetate, washed with brine, dried over $\mathrm{Na}_{2} \mathrm{SO}_{4}$, filtered, concentrated and purified with silica gel chromatography.

When the reaction was conducted at $80{ }^{\circ} \mathrm{C}$, only trace amount of $\mathbf{3 d}$ was obtained due to the decomposition of copper complex 12.

However, when the reaction was conducted at room temperature, $S$-3d was obtained. The product (125.8 mg, 45\% yield) as a white solid was purified with silica gel chromatography (Petroleum ether / EtOAc 50:1). S-3d: $[\alpha]^{26}{ }_{\mathrm{D}}=+0.15\left(c=1.0, \mathrm{CHCl}_{3}\right)$ for a $98 \%$ ee sample. Enantiomeric purity was determined by chiral HPLC analysis (Cellulose-1, flow $0.7 \mathrm{~mL} / \mathrm{min}, \mathrm{CH}_{3} \mathrm{CN}: \mathrm{H}_{2} \mathrm{O}=80: 20,214 \mathrm{~nm}$, $\mathrm{t}_{\mathrm{R}}$ $=11.406 \min$ (major) and $t_{R}=13.022$ (minor).

The absolute configuration of $S$-3d was characterized by X-ray crystallographic analysis (Figure S1). The crystal of $S$-3d was obtained by recrystallization from $n$-pentane and methanol at room temperature.

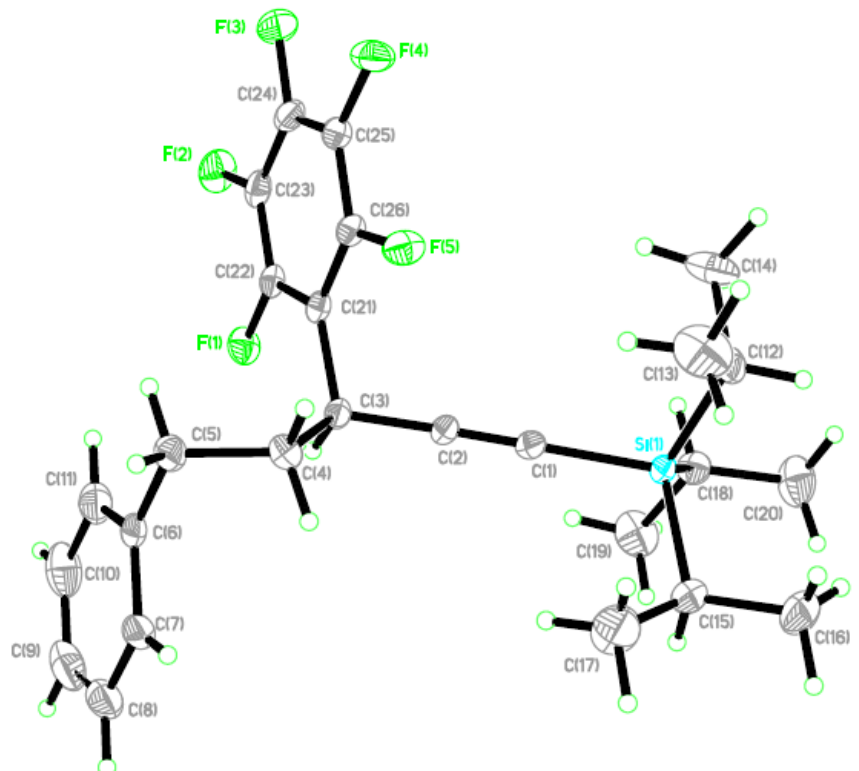

Figure S1. X-Ray Crystal Structure of Compound S-3d 


\section{Scheme S2. Reaction of $\boldsymbol{R}-2 \mathrm{~d}$ with 1a catalyzed by [Cu]}

a)<smiles>COC(=O)O[C@@H](C#C[In])CCc1ccccc1</smiles>

R-2d, (99\% ee)

b)<smiles>CO[Po](C#C[In])CCc1ccccc1</smiles>

$R-2 d,(99 \%$ ee)

c)

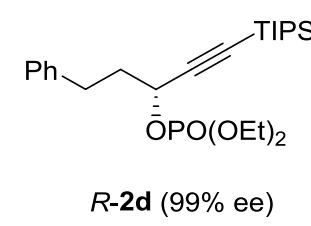<smiles>Fc1cc(F)c(F)c(F)c1F</smiles>

1a<smiles>Fc1cc(F)c(F)c(F)c1F</smiles>

1 a

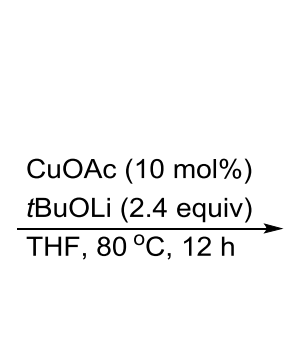
$\underset{\text { THF }, 80^{\circ} \mathrm{C}, 12 \mathrm{~h}}{\stackrel{t \text { BuLi }(2.4 \text { equiv })}{\longrightarrow}}$ $\mathrm{C}_{6} \mathrm{~F}_{5} \mathrm{Cu}$. Dioxane
$(5 \mathrm{~mol} \%)$
$\underset{\text { tBuOLi }(2.4 \text { equiv })}{\longrightarrow}$
THF, $80^{\circ} \mathrm{C}, 12 \mathrm{~h}$
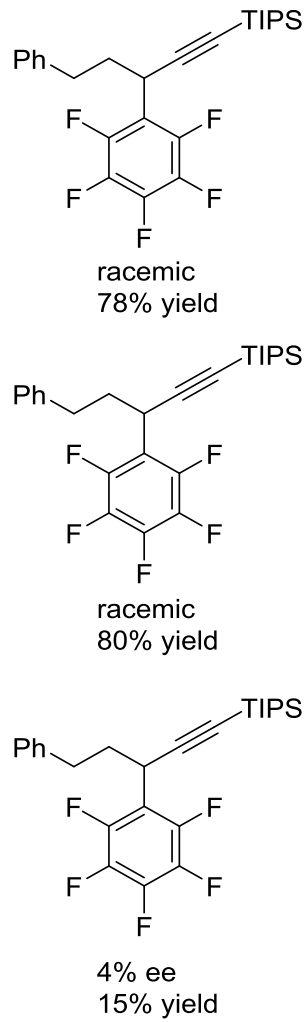

To a septum capped $25 \mathrm{~mL}$ of Schlenck tube were added CuOAc (0.06 mmol, $10 \mathrm{~mol} \%)$ or $\left(\mathrm{C}_{6} \mathrm{~F}_{5} \mathrm{Cu}\right)_{2}$-dioxane $(0.03 \mathrm{mmol}, 5 \mathrm{~mol} \%)$, and $t \mathrm{BuOLi}\left(1.44 \mathrm{mmol} 2.4\right.$ equiv) under $\mathrm{N}_{2}$, followed by THF (2.0 mL), $\boldsymbol{R}$-2d (0.6 mmol, 1.0 equiv), and $\mathrm{C}_{6} \mathrm{~F}_{5} \mathrm{H}(1.8 \mathrm{mmol} 3.0$ equiv). The tube was screw capped and the reaction was performed at $80{ }^{\circ} \mathrm{C}$ or room temperature. After stirring for $12 \mathrm{~h}$, the reaction mixture was cooled to room temperature, and diluted with ethyl acetate, washed with brine, dried over $\mathrm{Na}_{2} \mathrm{SO}_{4}$, filtered, concentrated and purified with silica gel chromatography. 


\section{Scheme S3. Reaction of $\mathrm{C}_{6} \mathrm{~F}_{5} \mathrm{Li}$ with $R-2 \mathrm{~d}$ or $S-3 \mathrm{~d}$}

a)

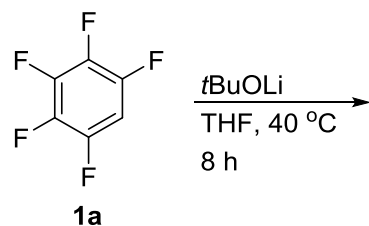

b)

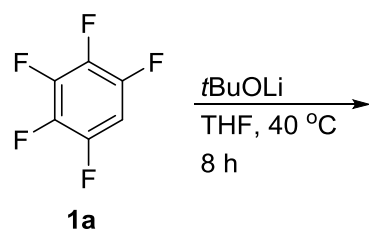

c)

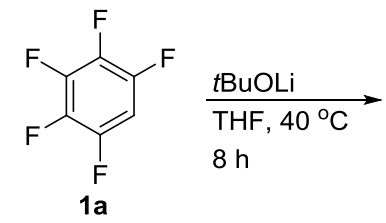<smiles>Fc1c(F)c(F)c(Cl)c(F)c1F</smiles><smiles>Oc1c(F)c(F)c(F)c(F)c1F</smiles>

$d-1 \mathbf{a} / \mathbf{1} \mathbf{a}>99 \%: 1$

$\mathrm{Ph}$

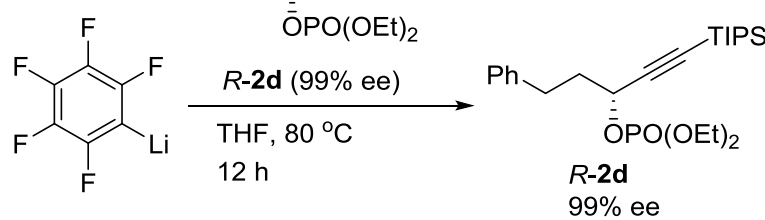

$99 \%$ ee

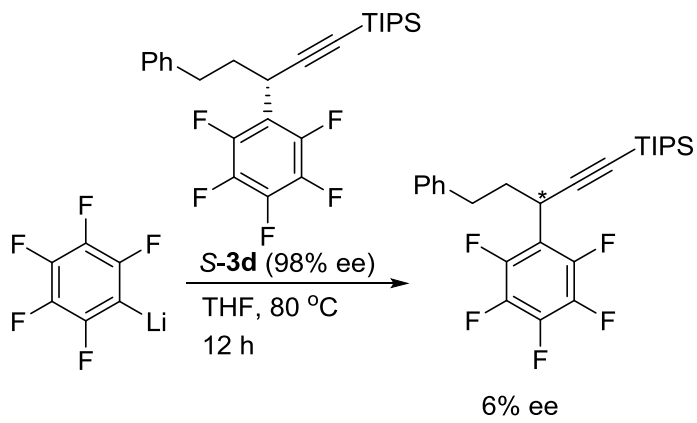

\section{Preparation of $\mathrm{C}_{6} \mathrm{~F}_{5} \mathrm{Li}$ (Scheme S3a)}

To a septum capped $25 \mathrm{~mL}$ of Schlenck tube were added $t \mathrm{BuOLi}\left(1.44 \mathrm{mmol} 2.4\right.$ equiv), $\mathrm{C}_{6} \mathrm{~F}_{5} \mathrm{H}$ (1.8 mmol 3.0 equiv), and THF ( $2.0 \mathrm{~mL})$ under $\mathrm{N}_{2}$, the tube was screw capped and the reaction was heated to $40{ }^{\circ} \mathrm{C}$ with stirring for $8 \mathrm{~h}$. The formation of $\mathrm{C}_{6} \mathrm{~F}_{5} \mathrm{Li}$ was confirmed by deuteration of $\mathrm{C}_{6} \mathrm{~F}_{5} \mathrm{Li}$ with $\mathrm{D}_{2} \mathrm{O}$ (2.0 equiv), in which $99 \%$ yield of $\mathrm{C}_{6} \mathrm{~F}_{5} \mathrm{D}$ was provided, suggesting that $\mathrm{C}_{6} \mathrm{~F}_{5} \mathrm{Li}$ was formed.

\section{Reaction of $\mathrm{C}_{6} \mathrm{~F}_{5} \mathrm{Li}$ with $\boldsymbol{R}-2 \mathrm{~d}$ or $S-3 \mathrm{~d}$ (Scheme S3b-c)}

To a septum capped $25 \mathrm{~mL}$ of Schlenck tube were added $t \mathrm{BuOLi}\left(1.44 \mathrm{mmol} 2.4\right.$ equiv), $\mathrm{C}_{6} \mathrm{~F}_{5} \mathrm{H}(1.8$ mmol 3.0 equiv), and THF (2.0 mL) under $\mathrm{N}_{2}$, the tube was screw capped and the reaction was stirred at $40{ }^{\circ} \mathrm{C}$ for $2 \mathrm{~h}$. The resulting solution was then cooled to room temperature, $R$-2d $(0.6$ mmol, 1.0 equiv) or $S \mathbf{- 3 d}$ ( $0.6 \mathrm{mmol}, 1.0$ equiv) was added. After the reaction mixture was stirred at $80{ }^{\circ} \mathrm{C}$ for 12 $\mathrm{h}$, the reaction was cooled to room temperature and the resulting sample was used for the ee determination. 
Scheme S4. Reaction of $t$ BuOLi with $R-2 d$ or $S-3 d$

a)
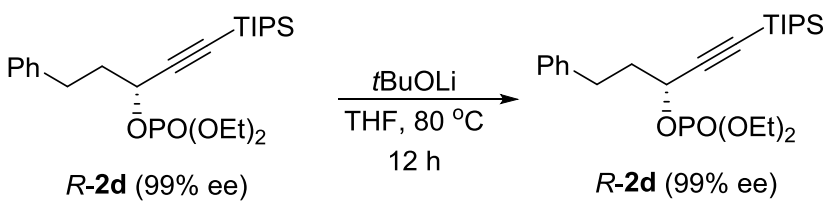

b)
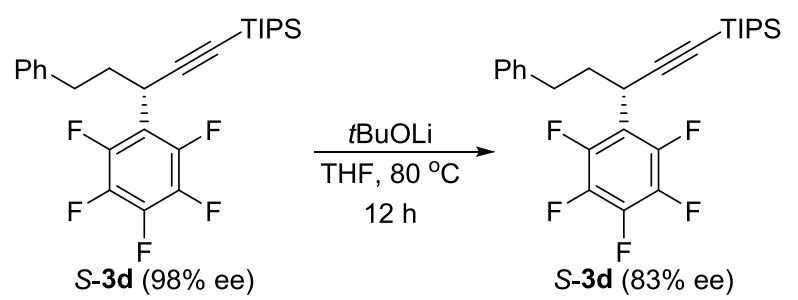

\section{Procedure:}

To a septum capped $25 \mathrm{~mL}$ of Schlenck tube were added $t$ BuOLi ( $0.1 \mathrm{mmol}, 1.0$ equiv), $R$-2d or $S$-3d (0.1 mmol, 1.0 equiv) and THF $(2.0 \mathrm{~mL})$ under $\mathrm{N}_{2}$. The tube was screw capped and the reaction was heated to $80{ }^{\circ} \mathrm{C}$ with stirring for $12 \mathrm{~h}$. The reaction was then cooled to room temperature and the resulting sample was used for the ee determination. 
Diethyl (1-(triisopropylsilyl)hept-1-yn-3-yl) phosphate (2a)
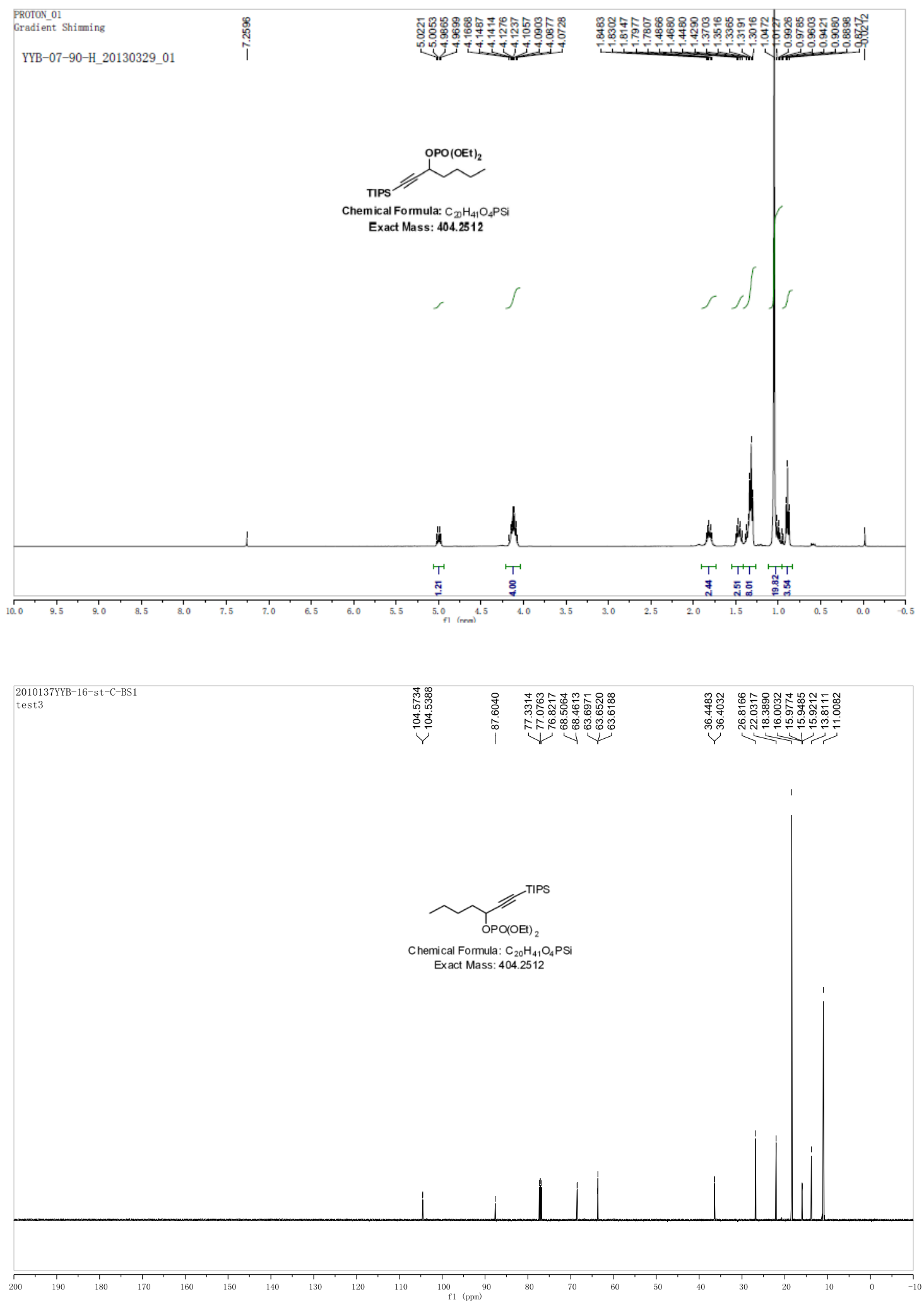
5-Cyclohexyl-1-(triisopropylsilyl)pent-1-yn-3-yl diethyl phosphate (2b)
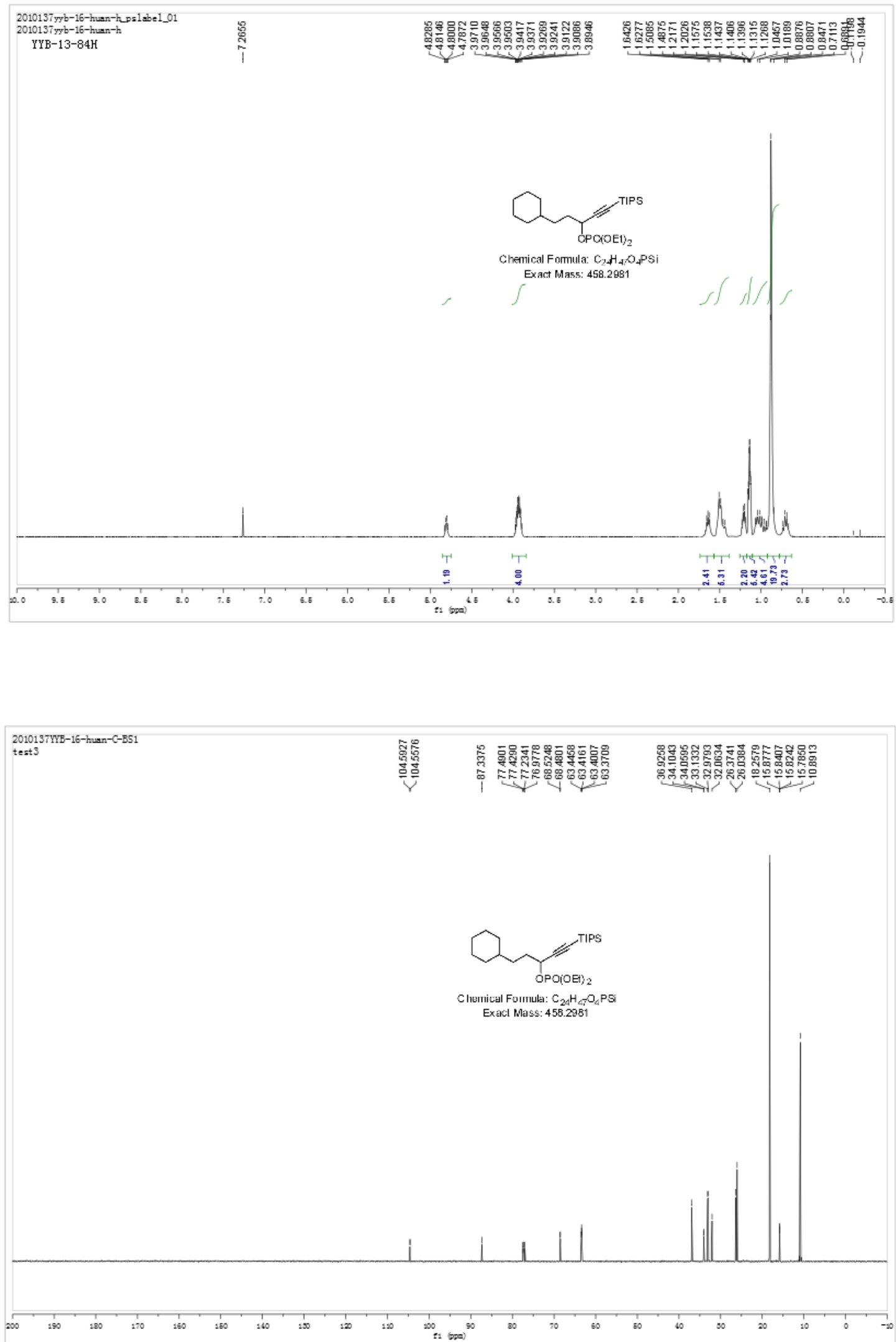
Diethyl (5-methyl-1-(triisopropylsilyl)hex-1-yn-3-yl) phosphate (2c)
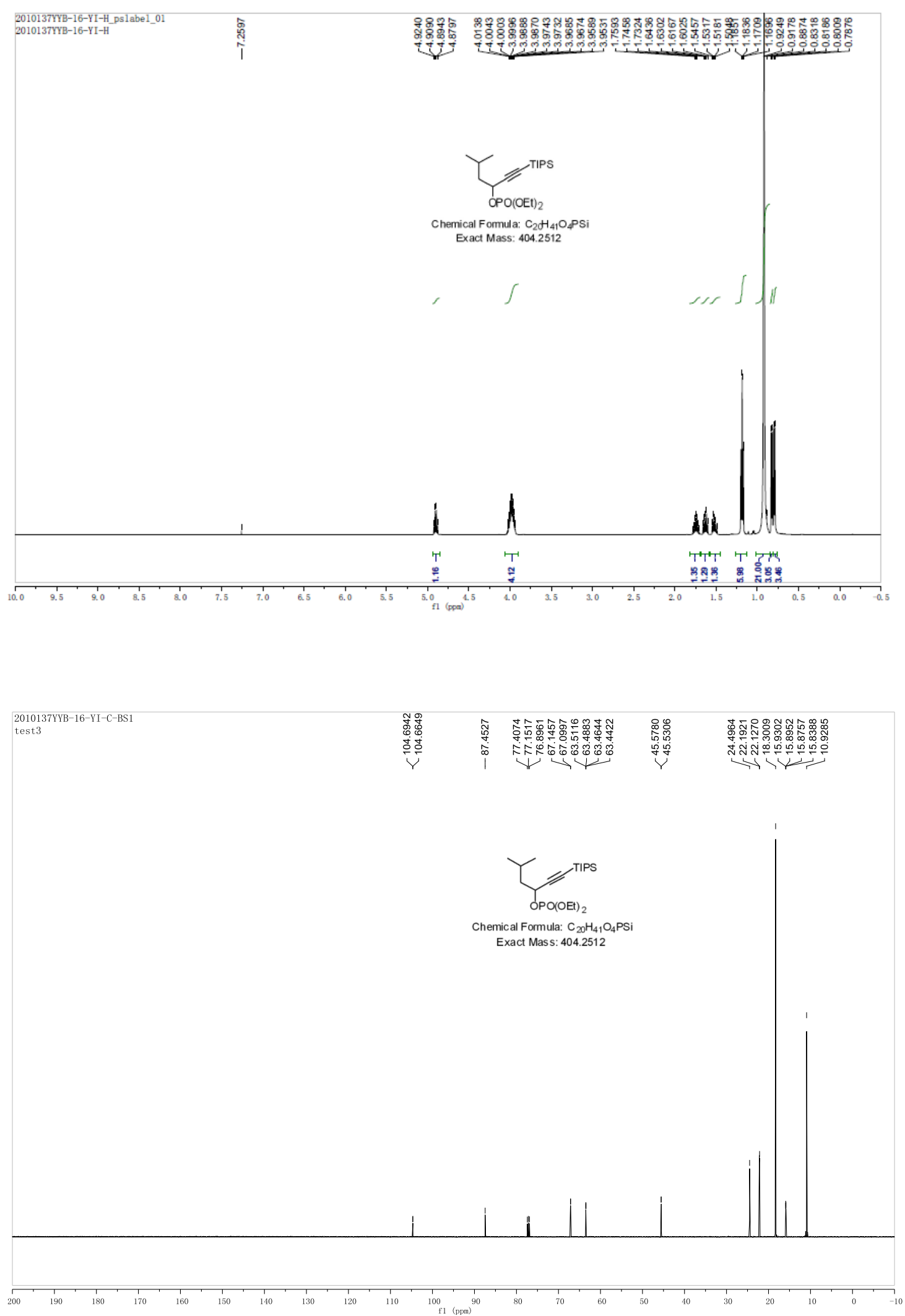
Diethyl (5-phenyl-1-(triisopropylsilyl)pent-1-yn-3-yl) phosphate (2d)
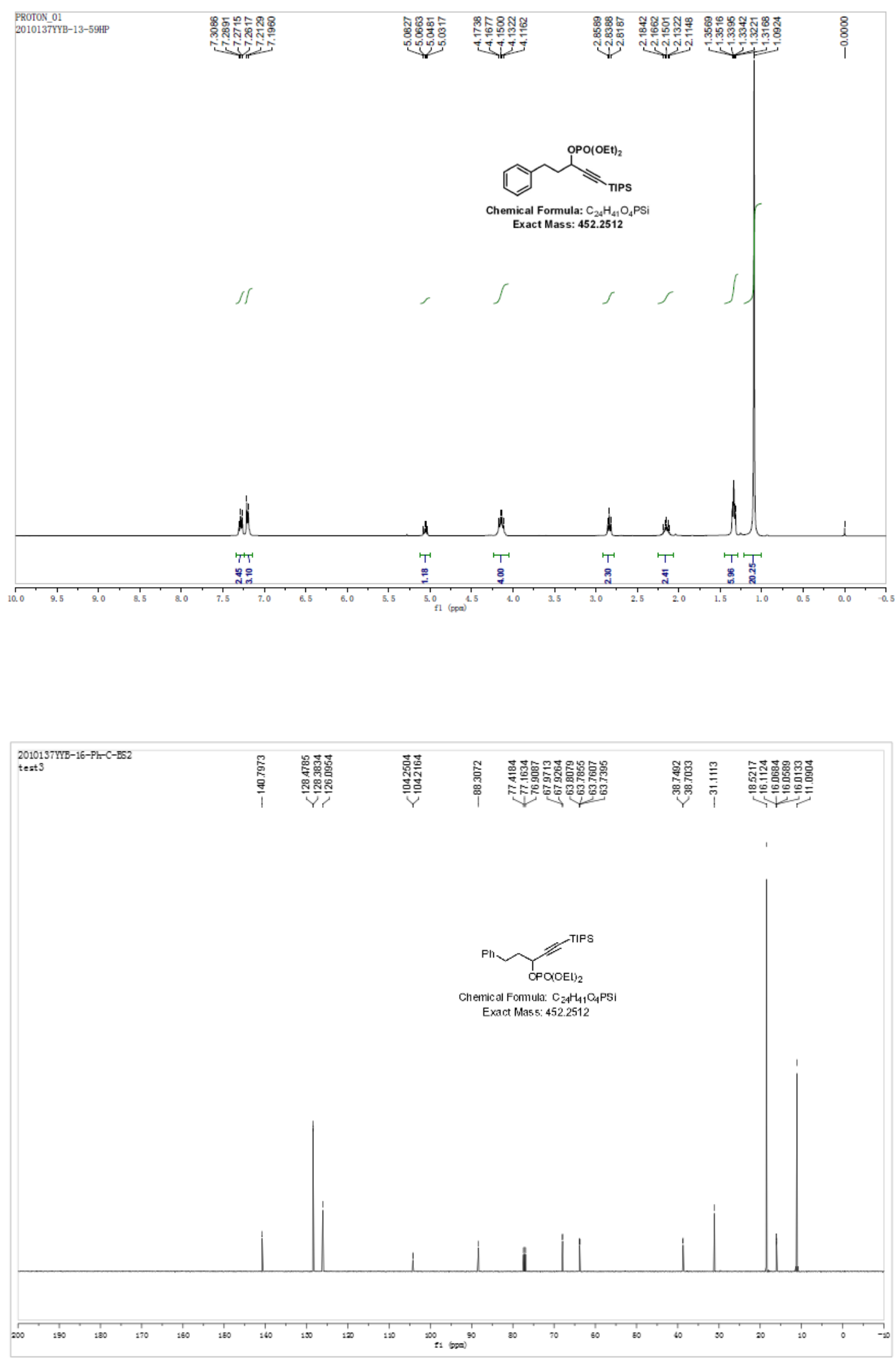
(Z)-Diethyl (1-(triisopropylsilyl)non-6-en-1-yn-3-yl) phosphate (2e)
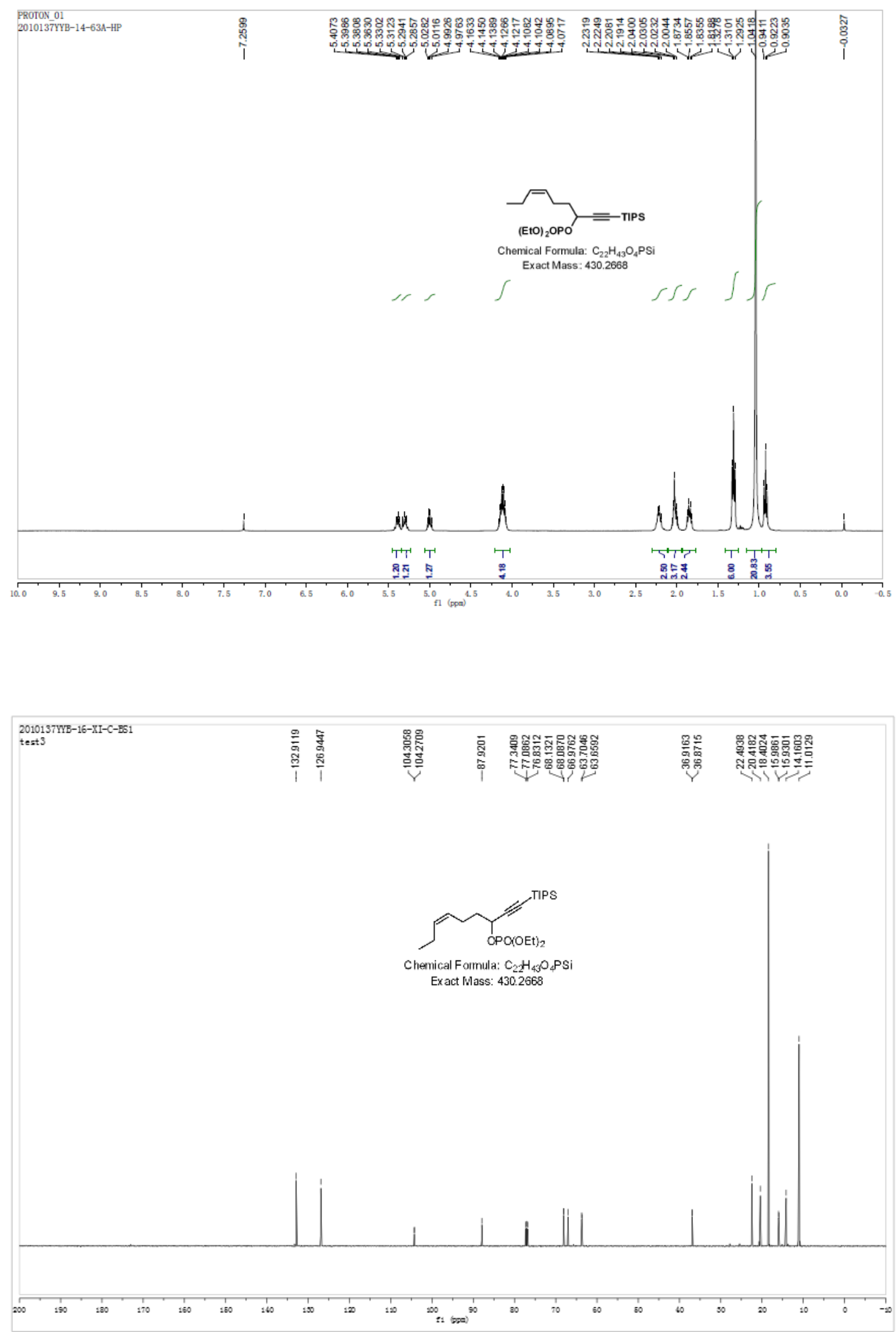
Diethyl (1-(triisopropylsilyl)tetradeca-1,11-diyn-3-yl) phosphate (2f)
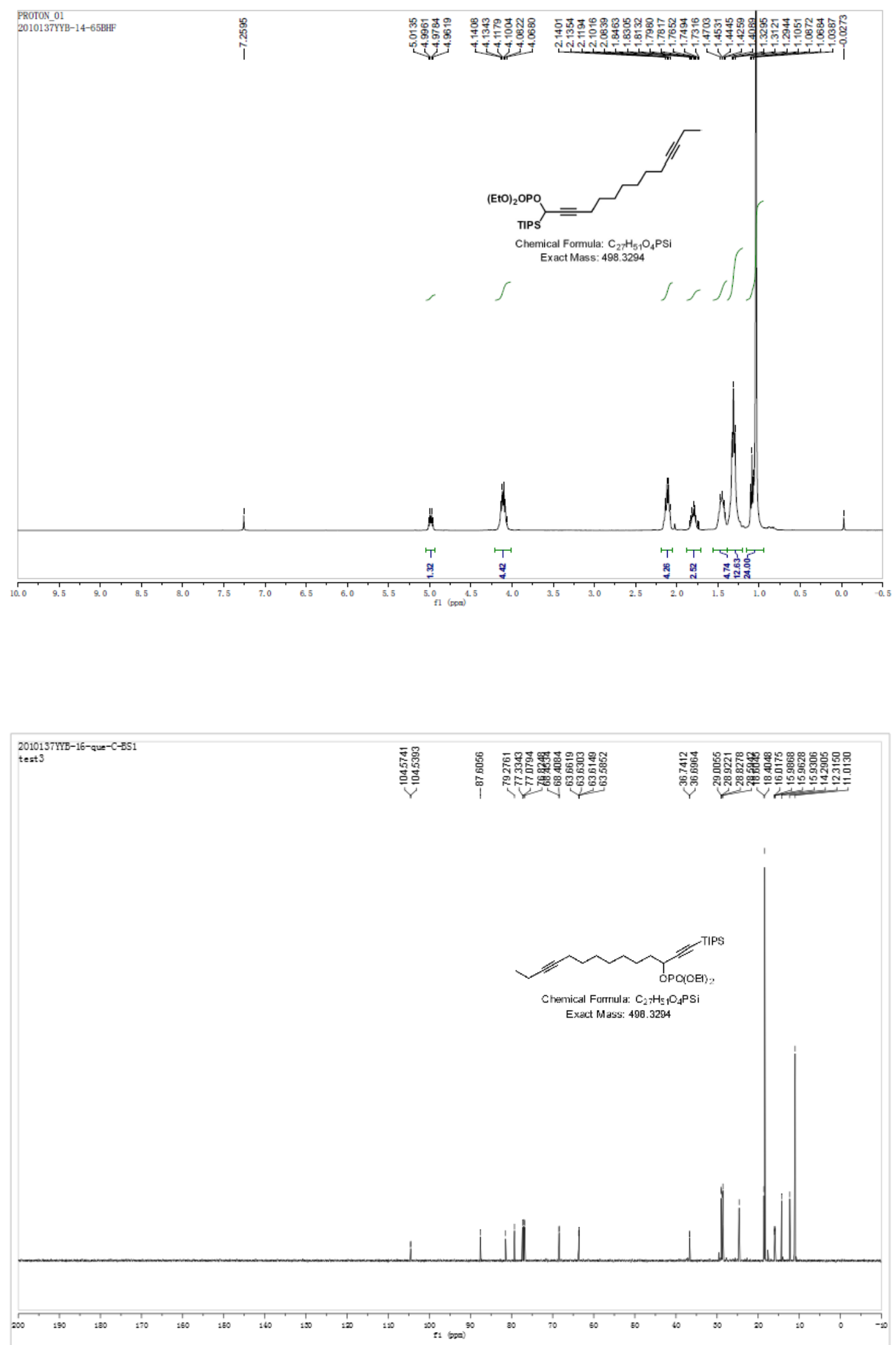
7-((tert-Butyldimethylsilyl)oxy)-1-(triisopropylsilyl)hept-1-yn-3-yl diethyl phosphate (2g)
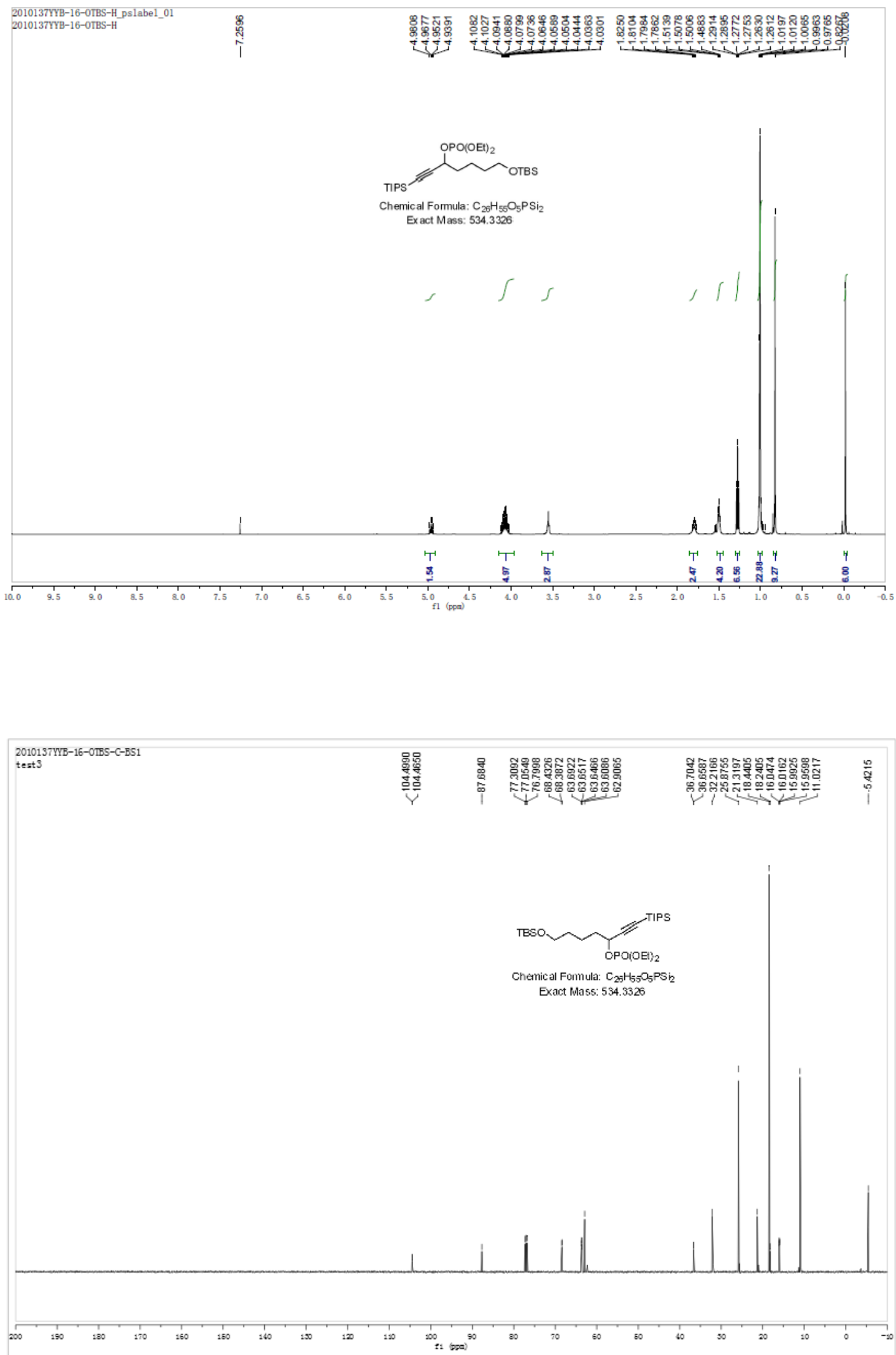
7-(Benzyloxy)-1-(triisopropylsilyl)hept-1-yn-3-yl diethyl phosphate (2h)
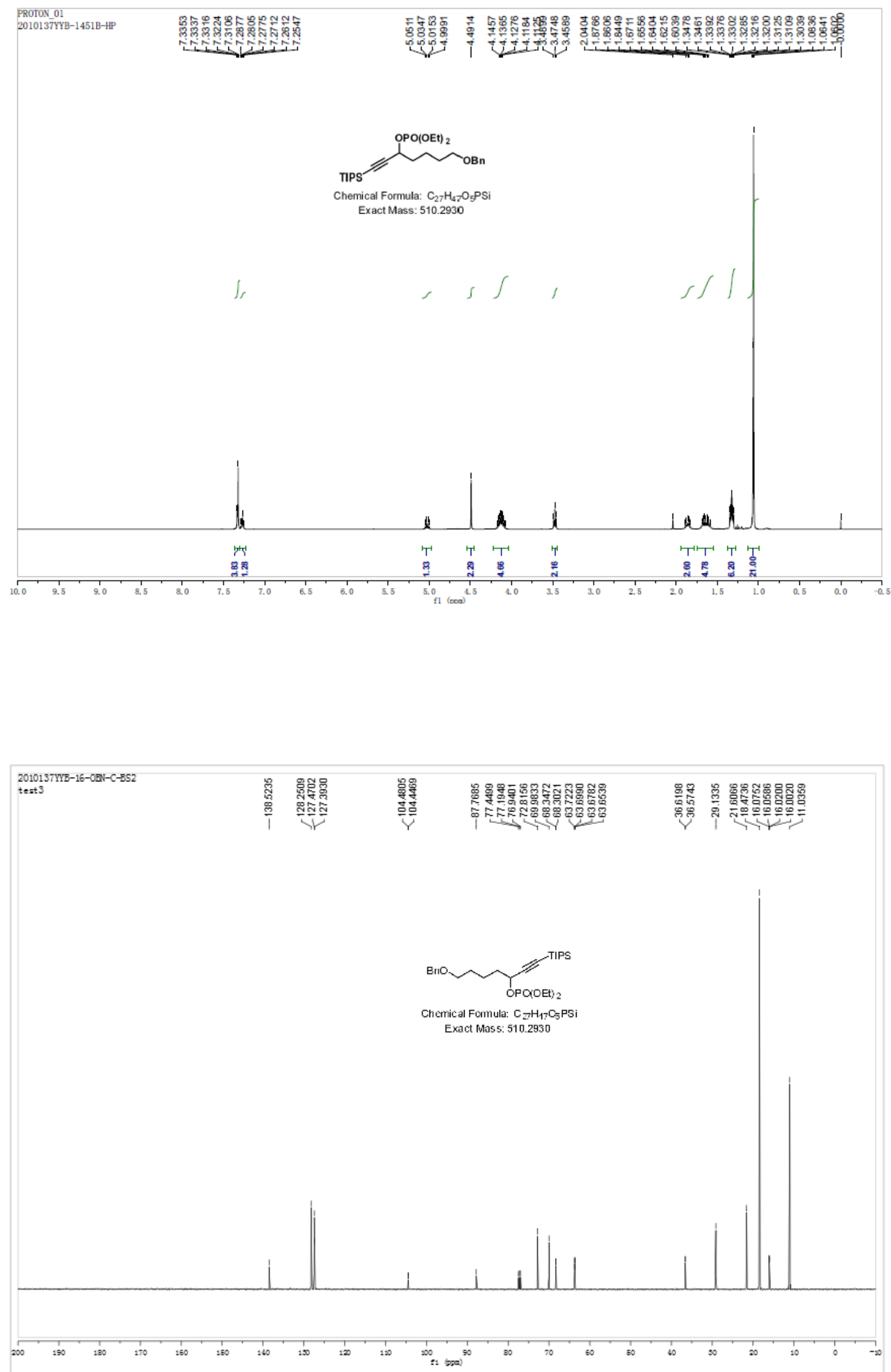
8-Bromo-1-(triisopropylsilyl)oct-1-yn-3-yl diethyl phosphate (2i)
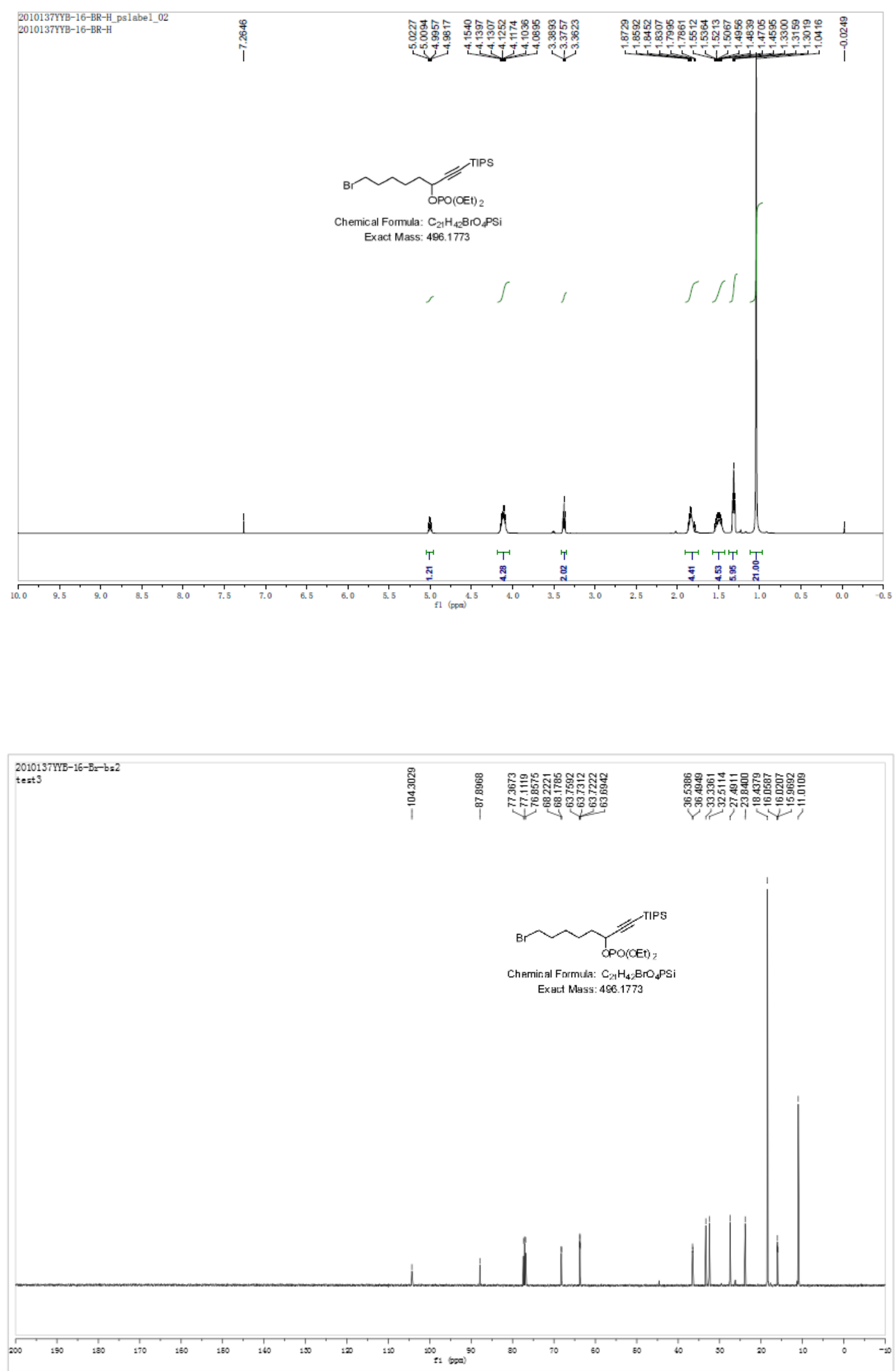
Diethyl (1-(triethylsilyl)hept-1-yn-3-yl) phosphate (2j)
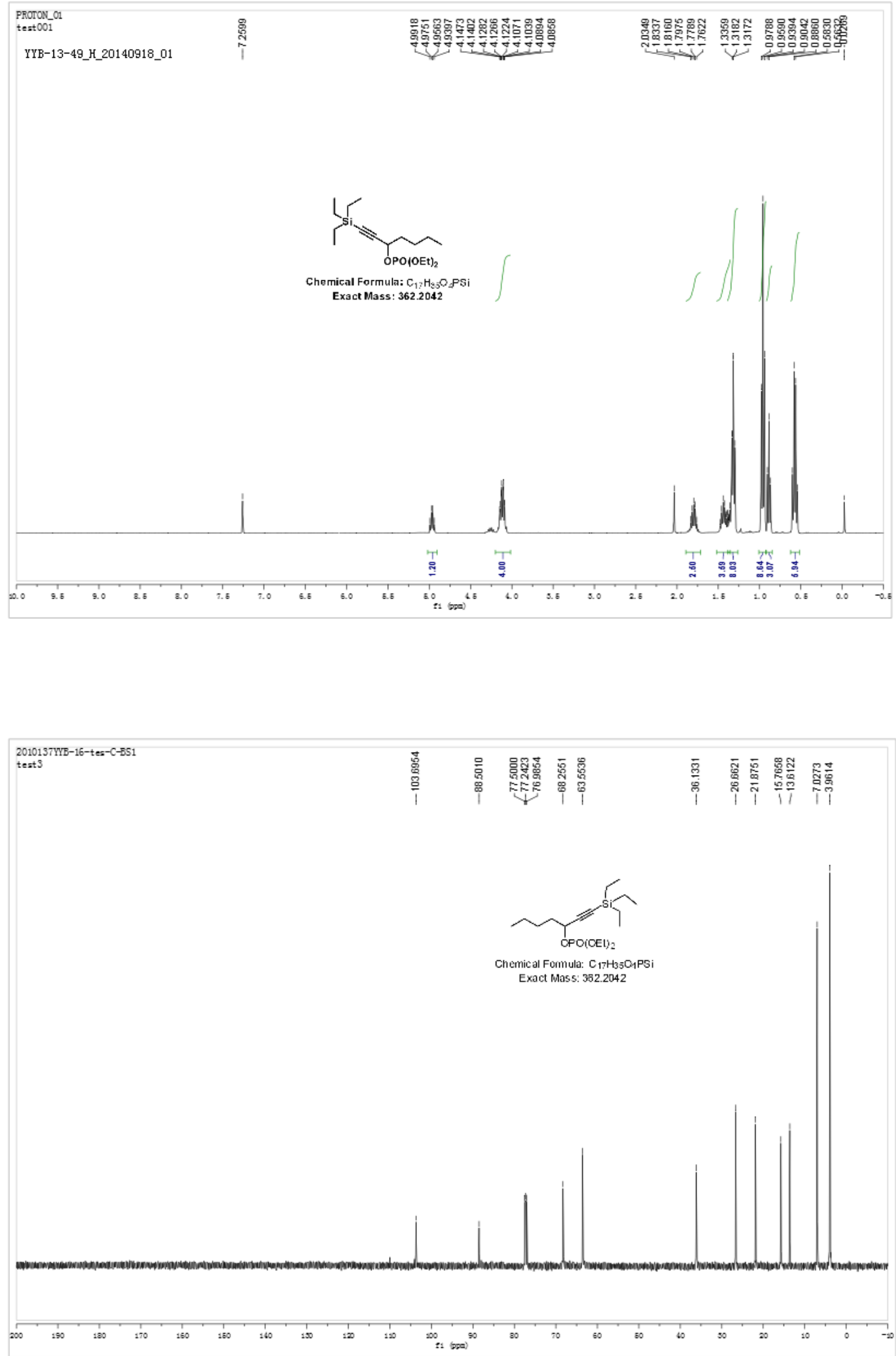
2,2-Dimethylnon-3-yn-5-yl diethyl phosphate (2k)
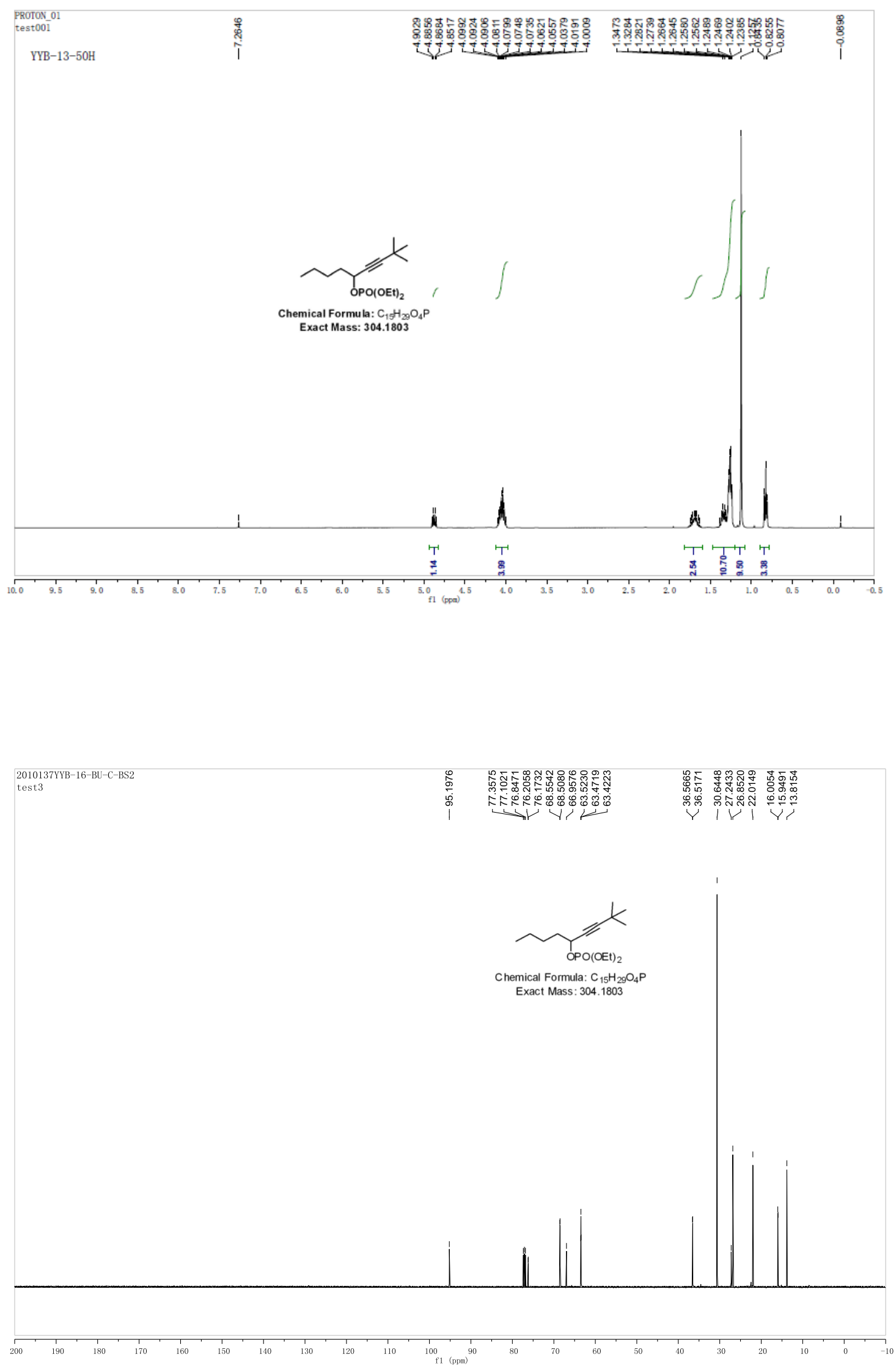
Triisopropyl(3-(perfluorophenyl)hept-1-yn-1-yl)silane (3a)
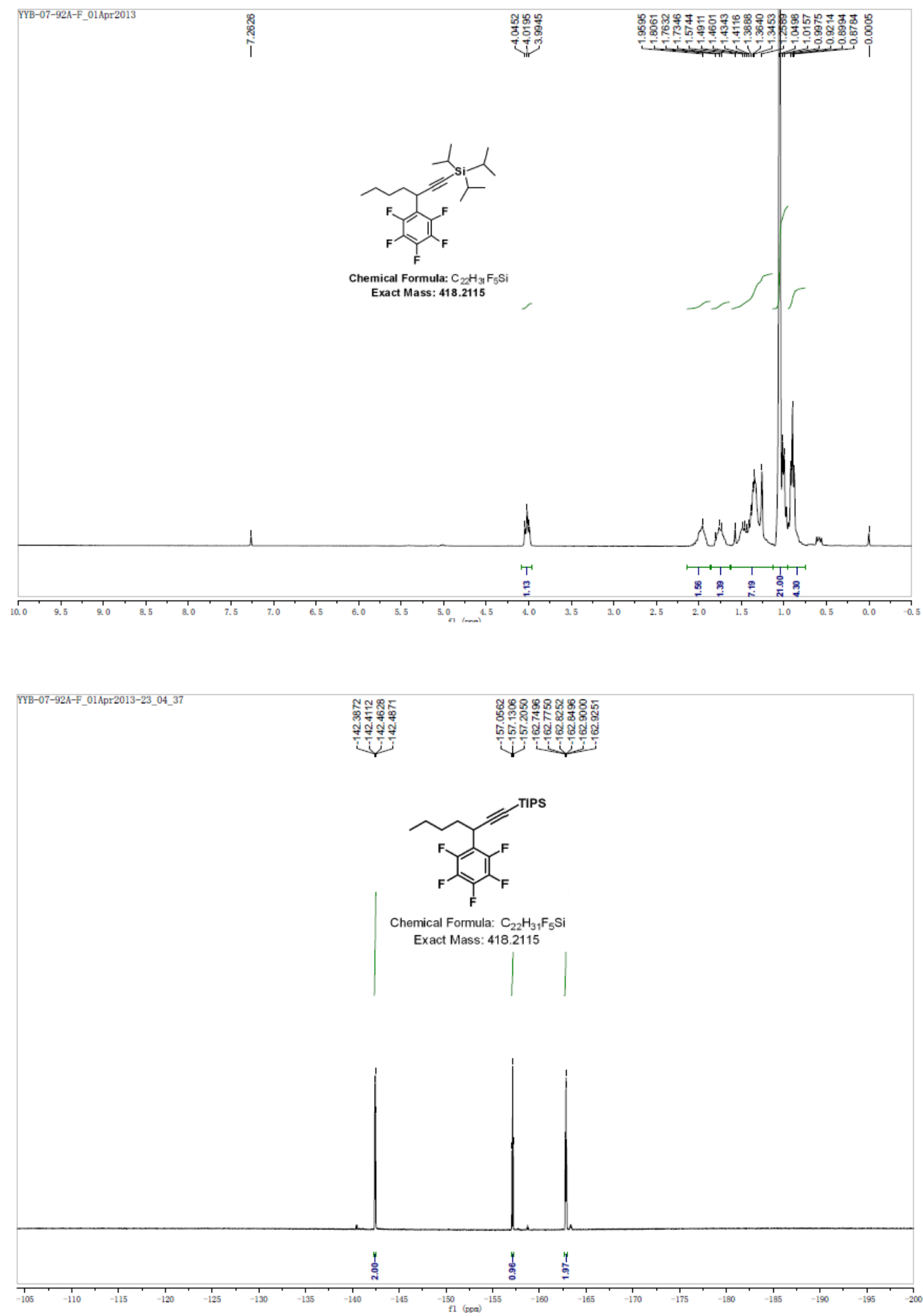


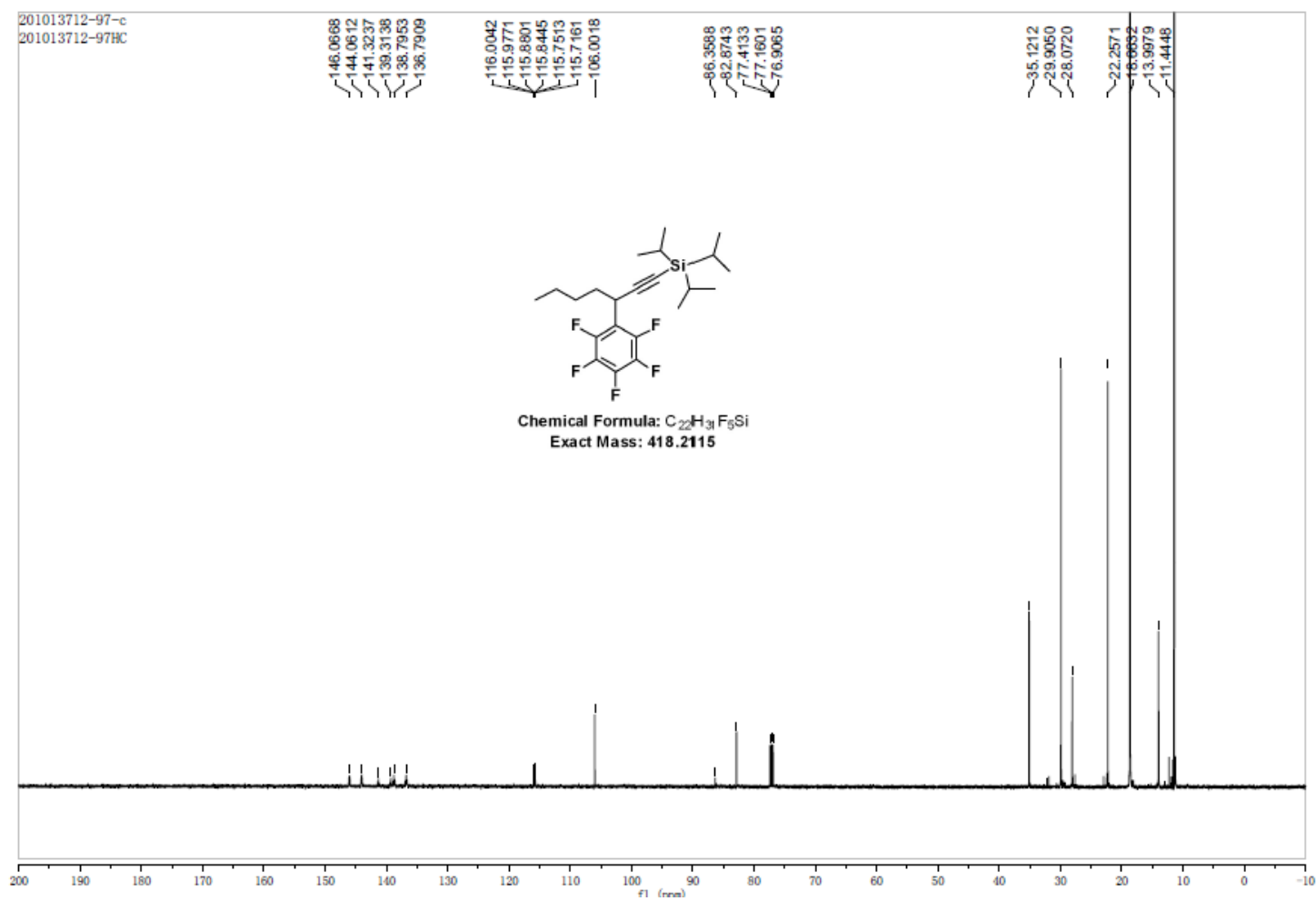

(5-Cyclohexyl-3-(perfluorophenyl)pent-1-yn-1-yl)triisopropylsilane (3b)

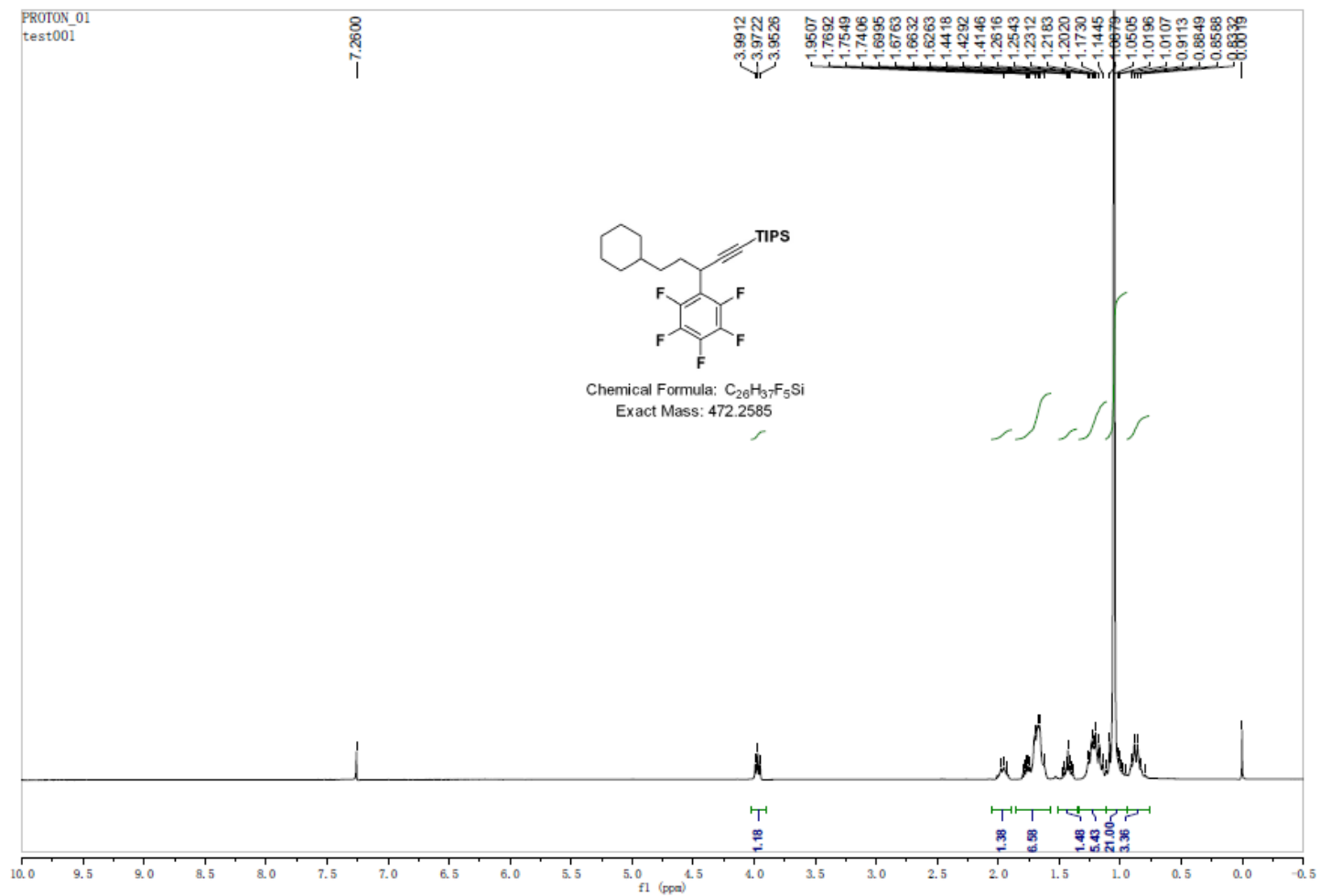



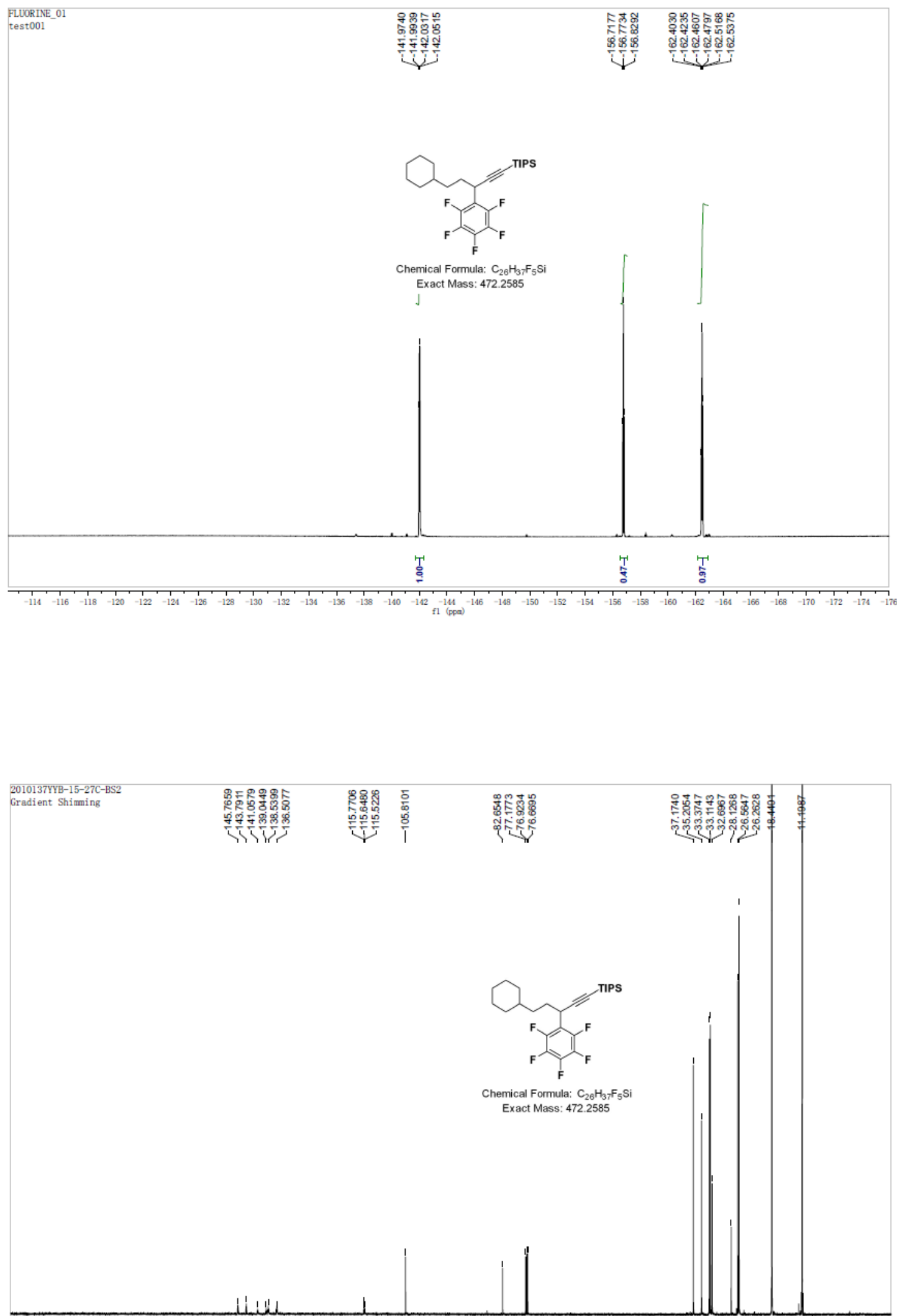

Iind 
Triisopropyl(5-methyl-3-(perfluorophenyl)hex-1-yn-1-yl)silane (3c)
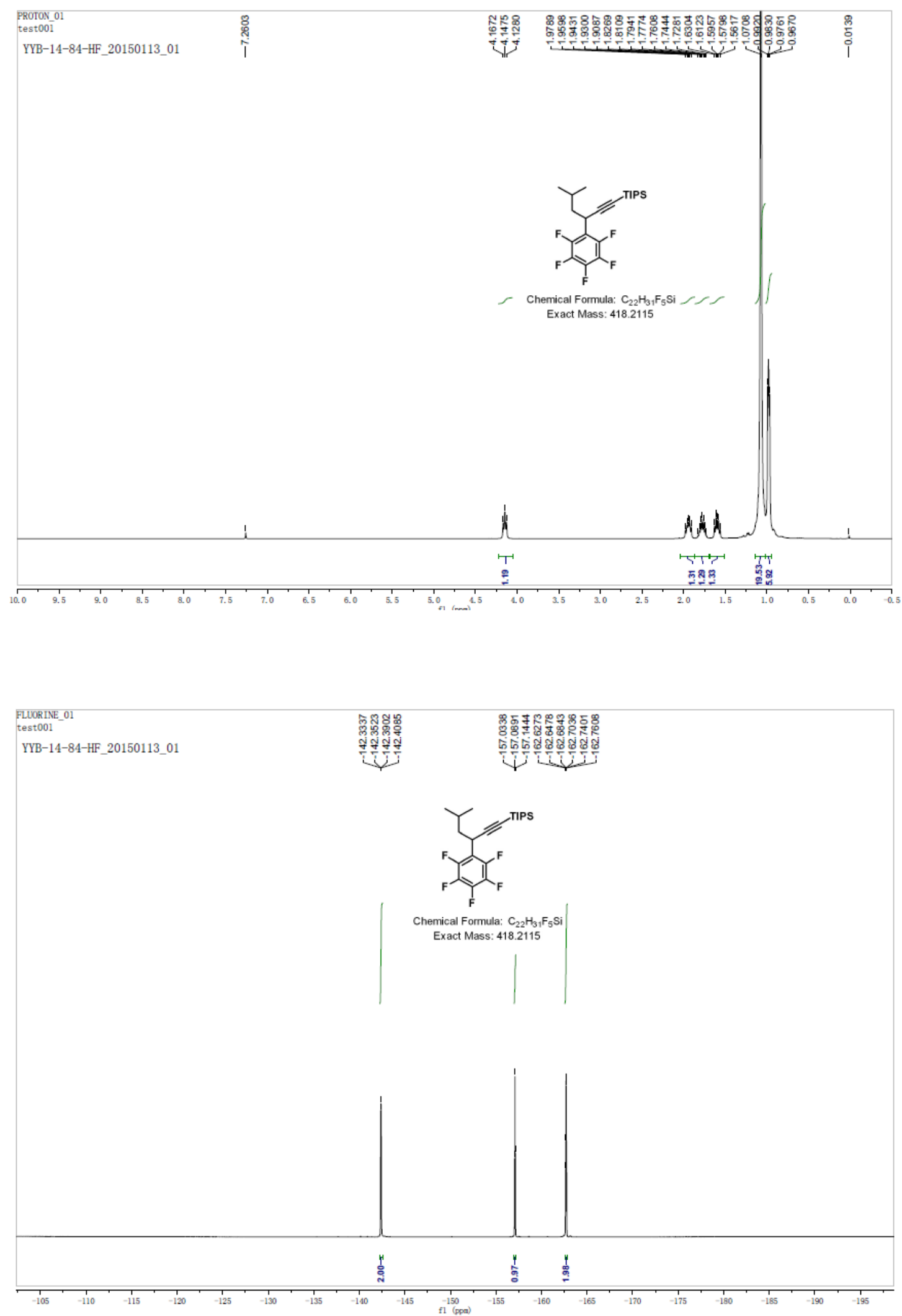


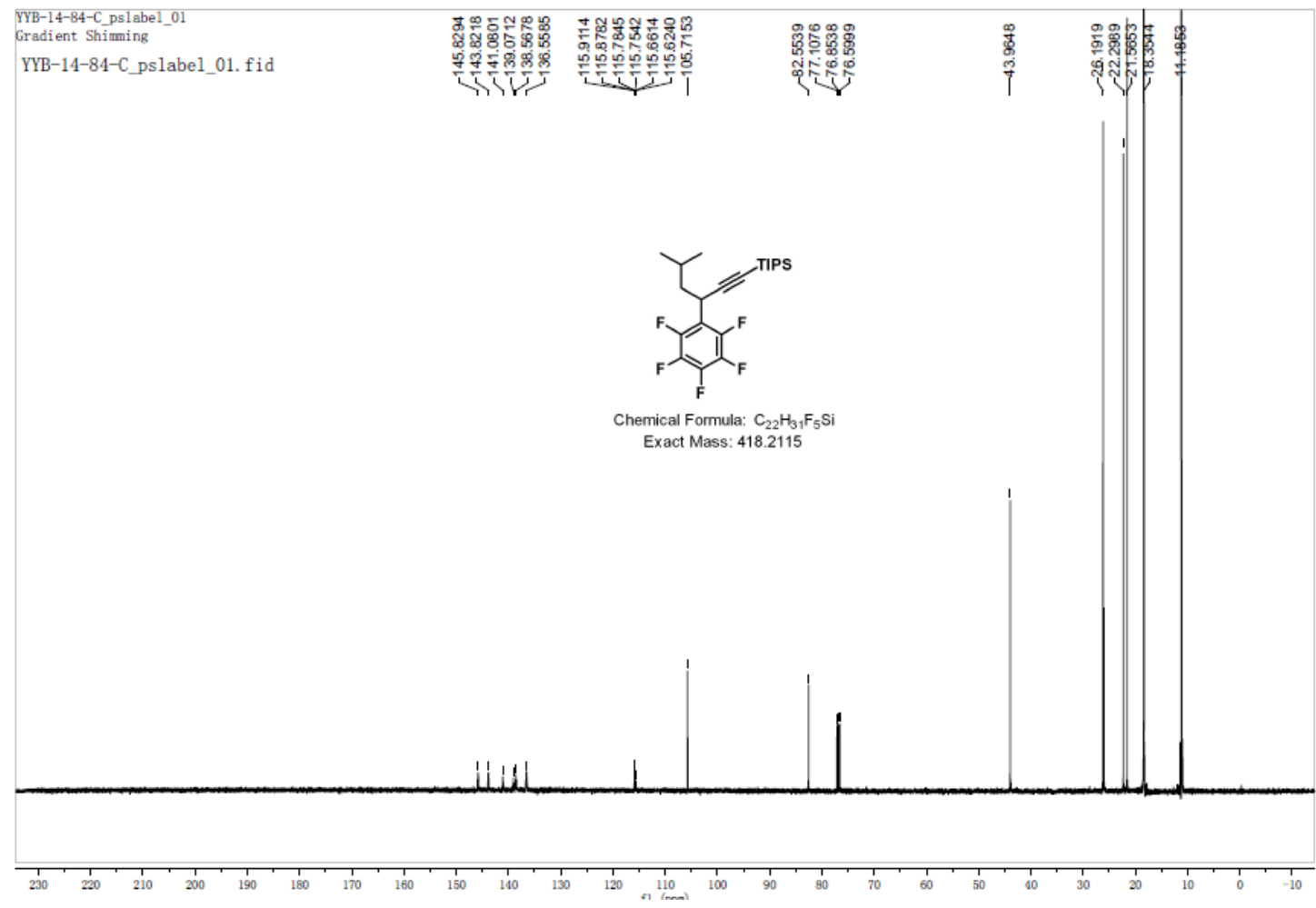

Triisopropyl(3-(perfluorophenyl)-5-phenylpent-1-yn-1-yl)silane (3d)

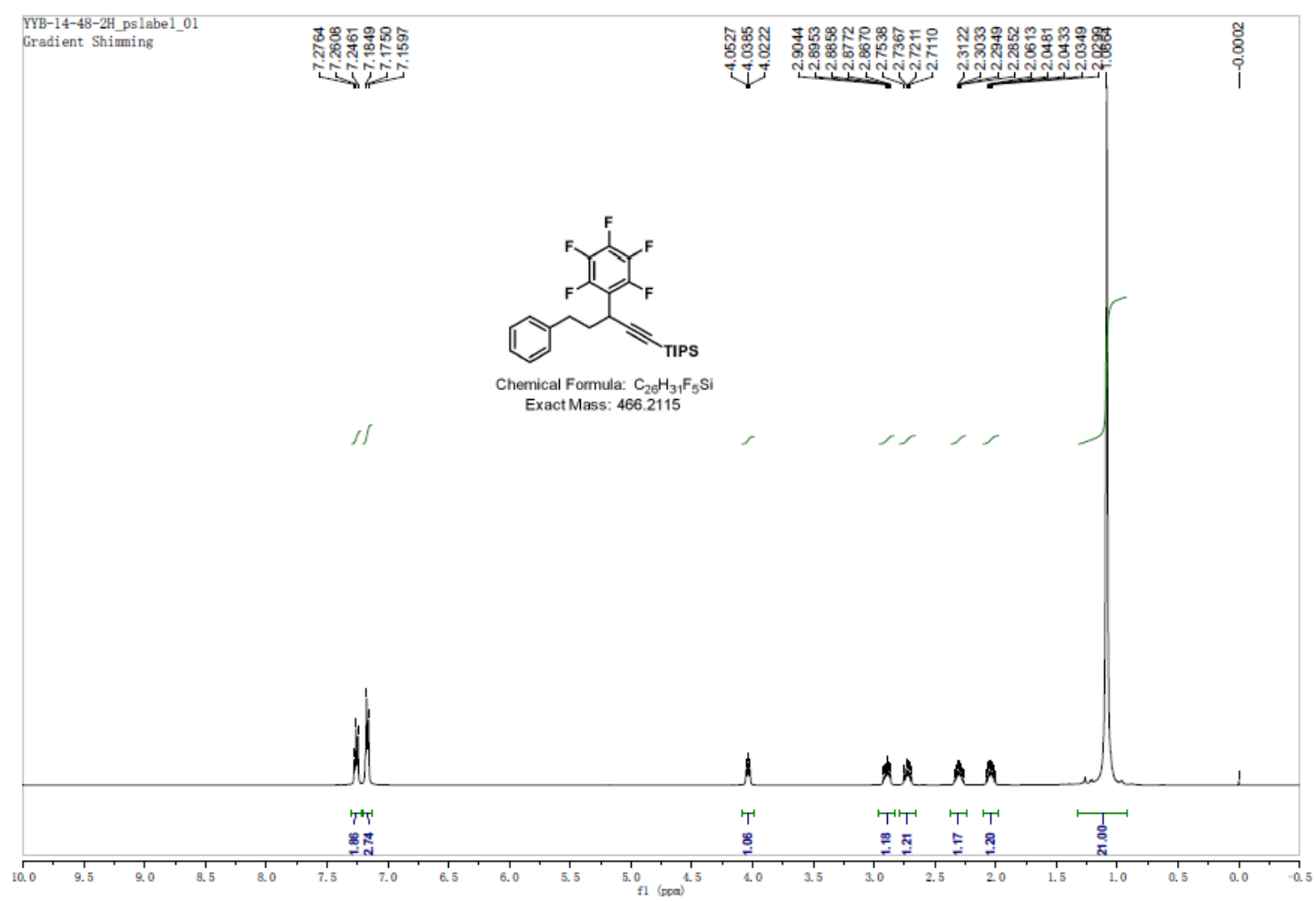



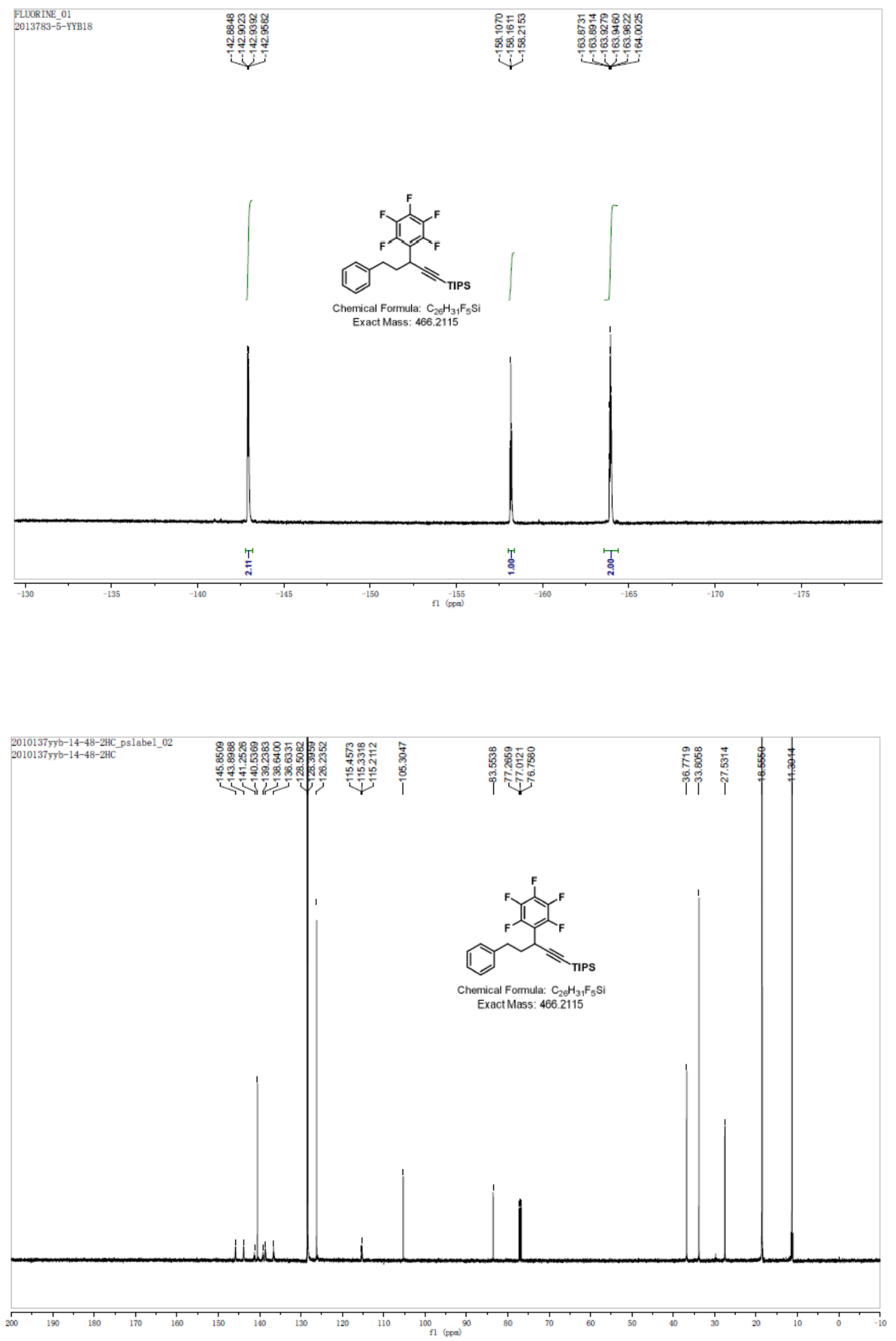
(Z)-Triisopropyl(3-(perfluorophenyl)non-6-en-1-yn-1-yl)silane (3e)

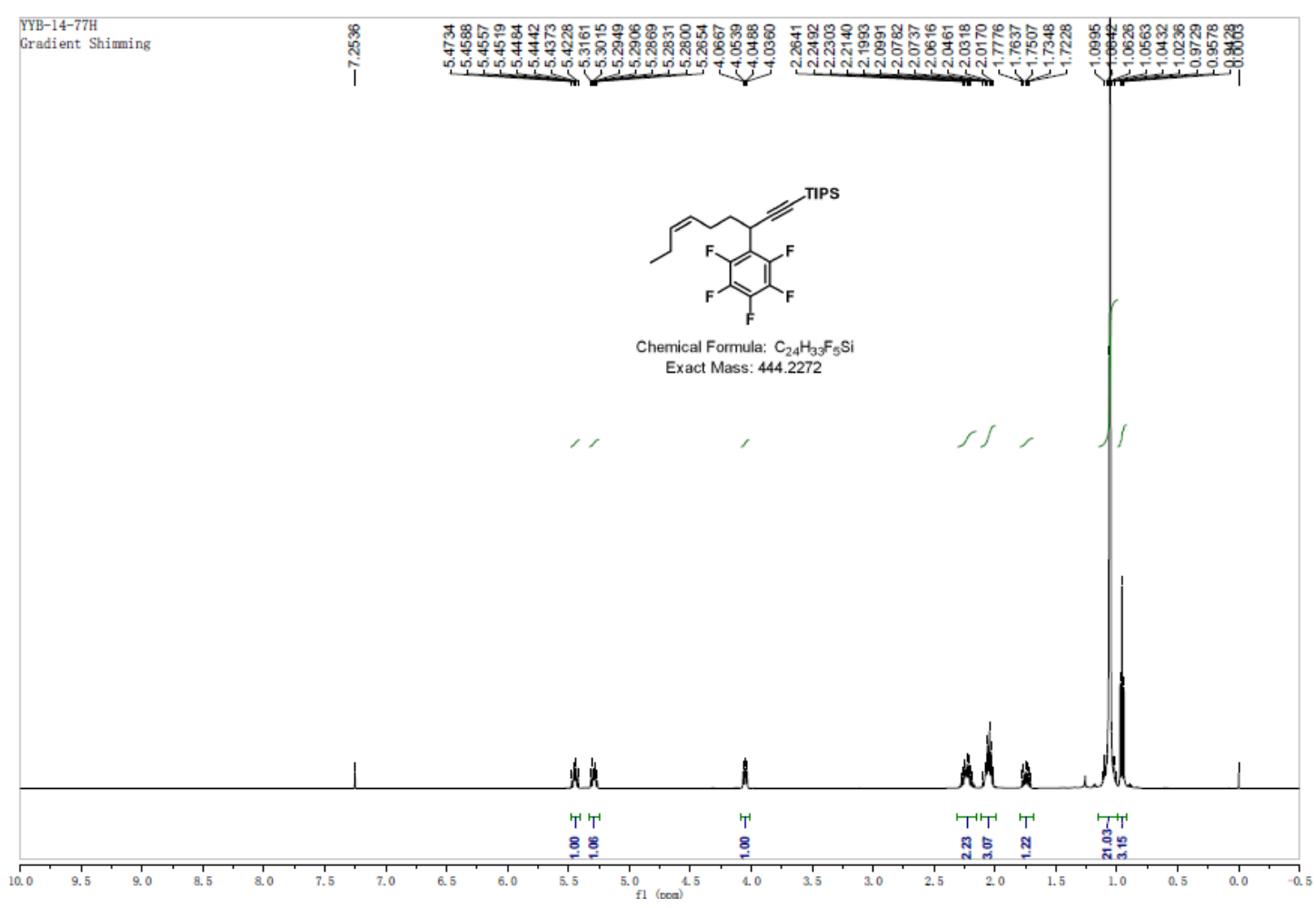

FLUORINE_01
2010137YYB-QUE-HF
YYB-14-77-F_20150110_01

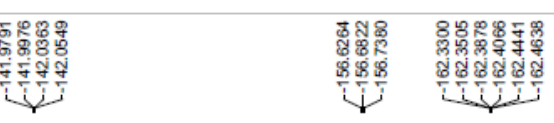

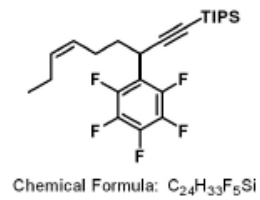

Exact Mass: 444.2272

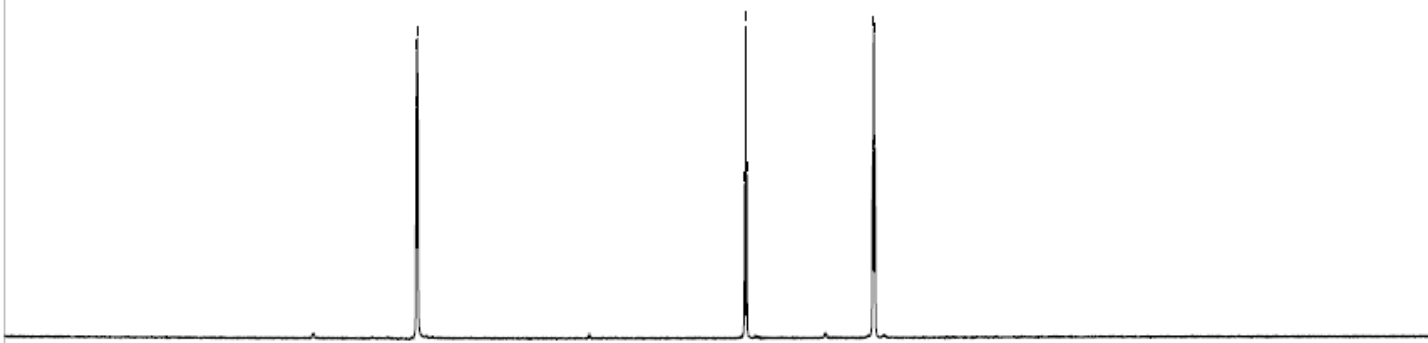

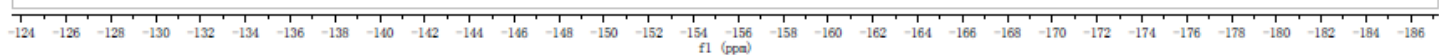




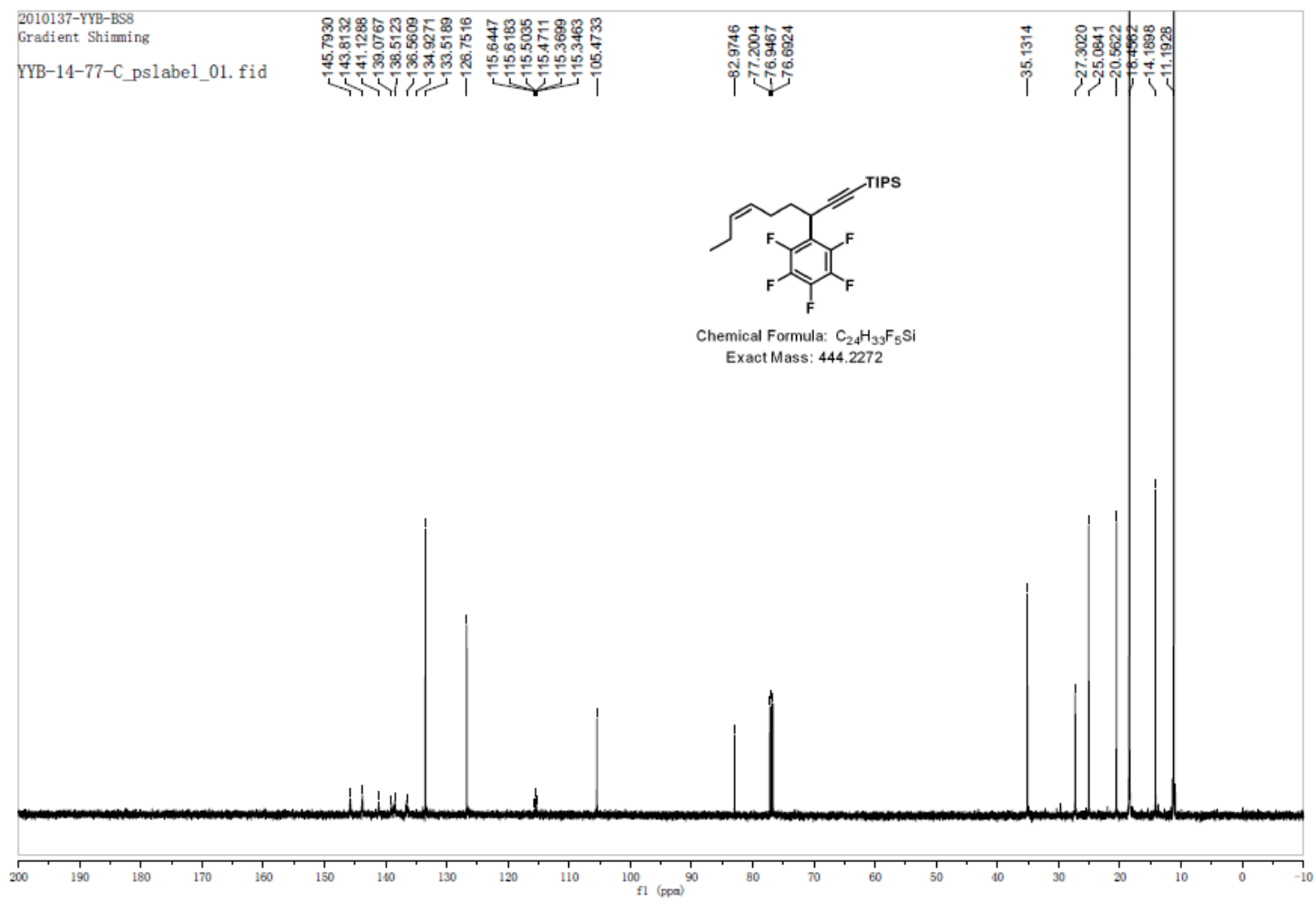

Triisopropyl(3-(perfluorophenyl)tetradeca-1,11-diyn-1-yl)silane (3f)

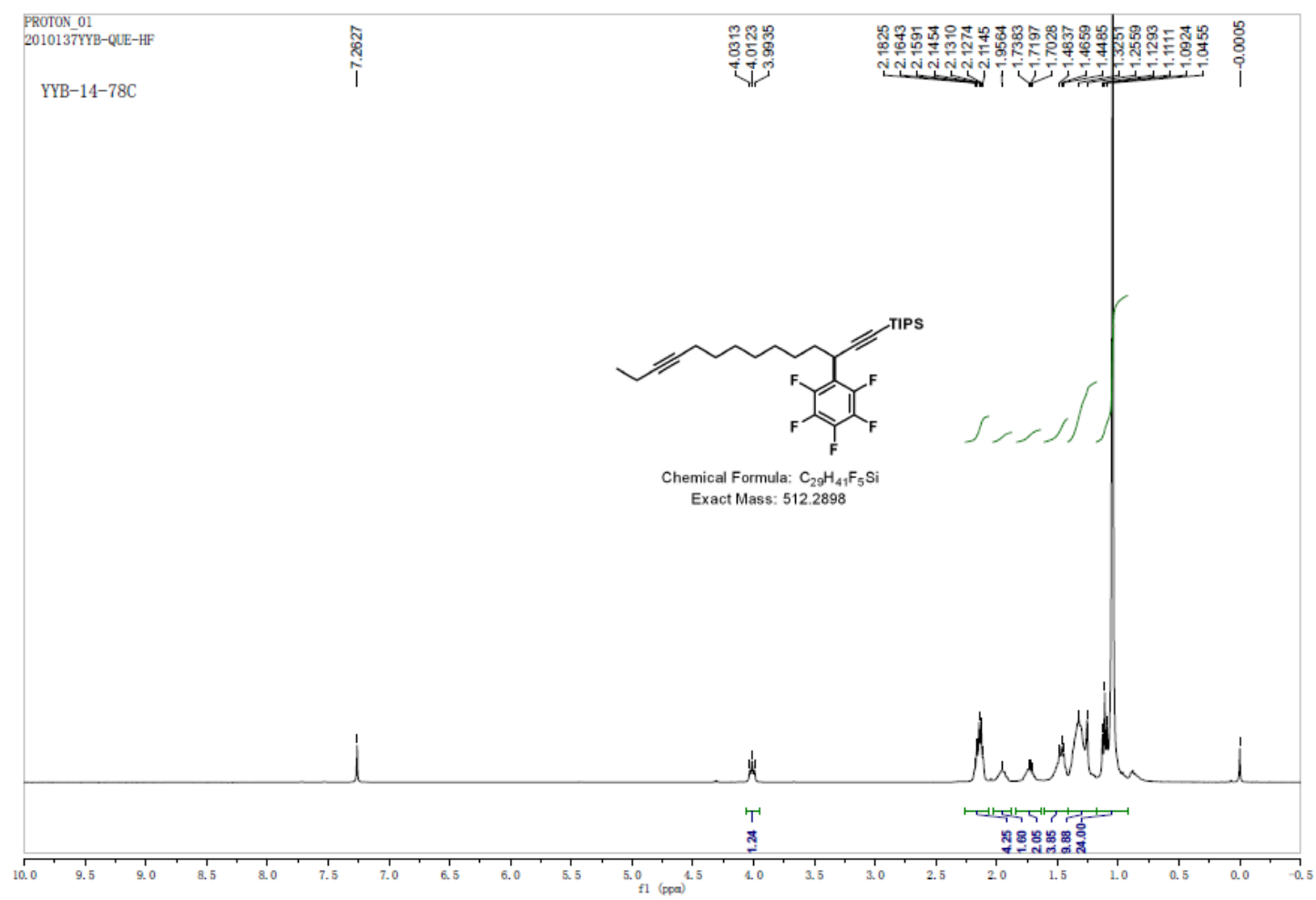



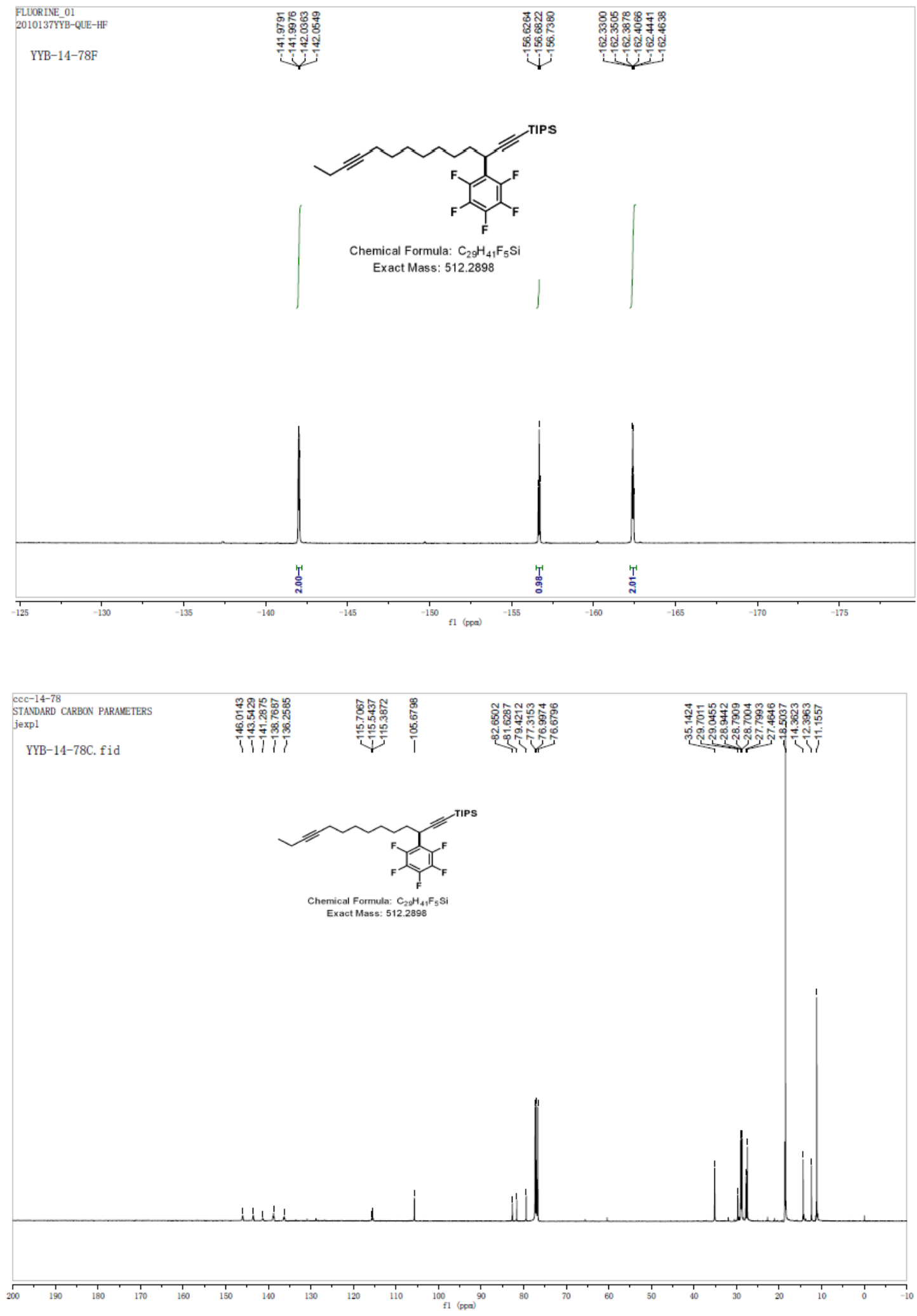
tert-Butyldimethyl((5-(perfluorophenyl)-7-(triisopropylsilyl)hept-6-yn-yl)oxy)silane (3g)
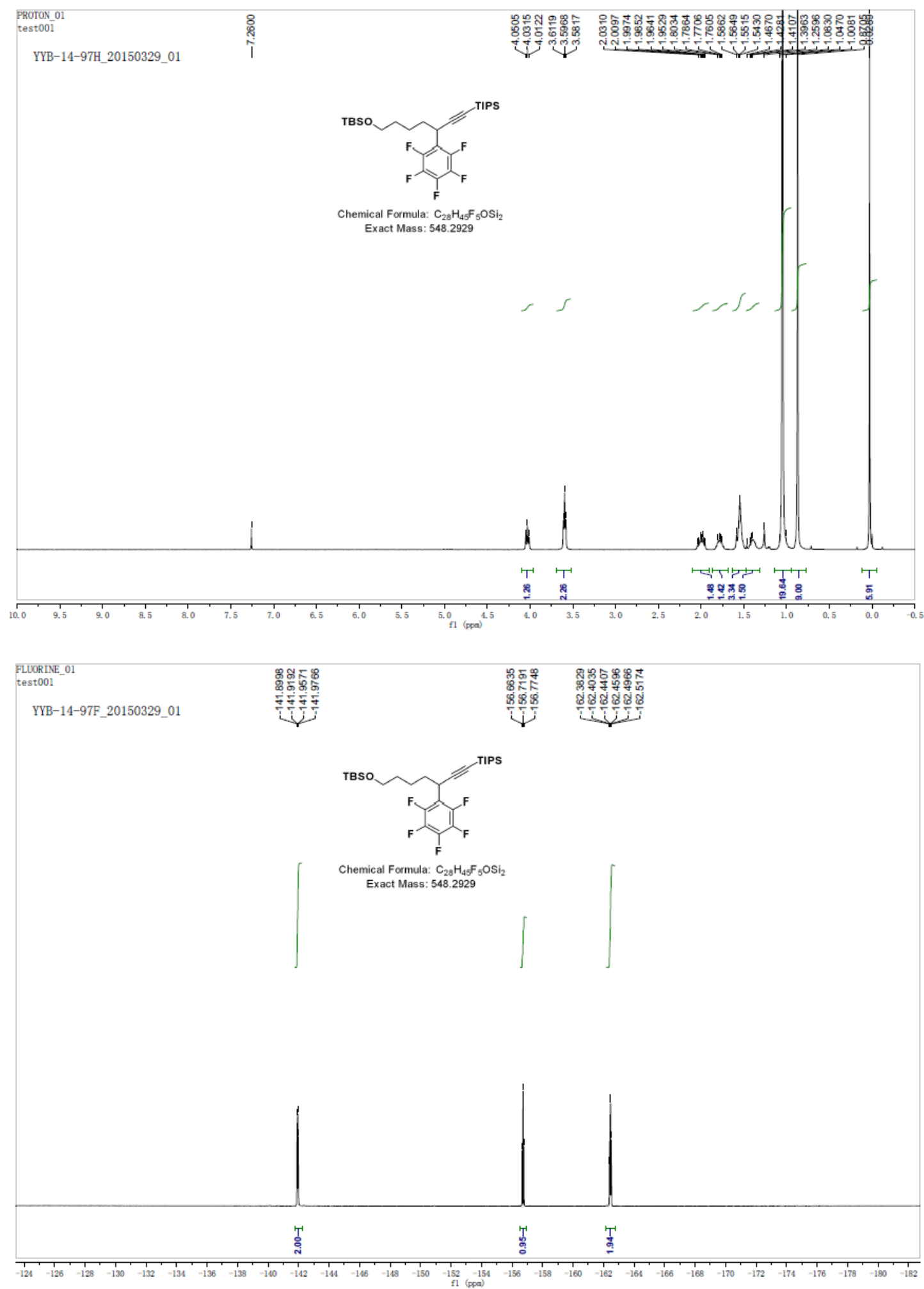


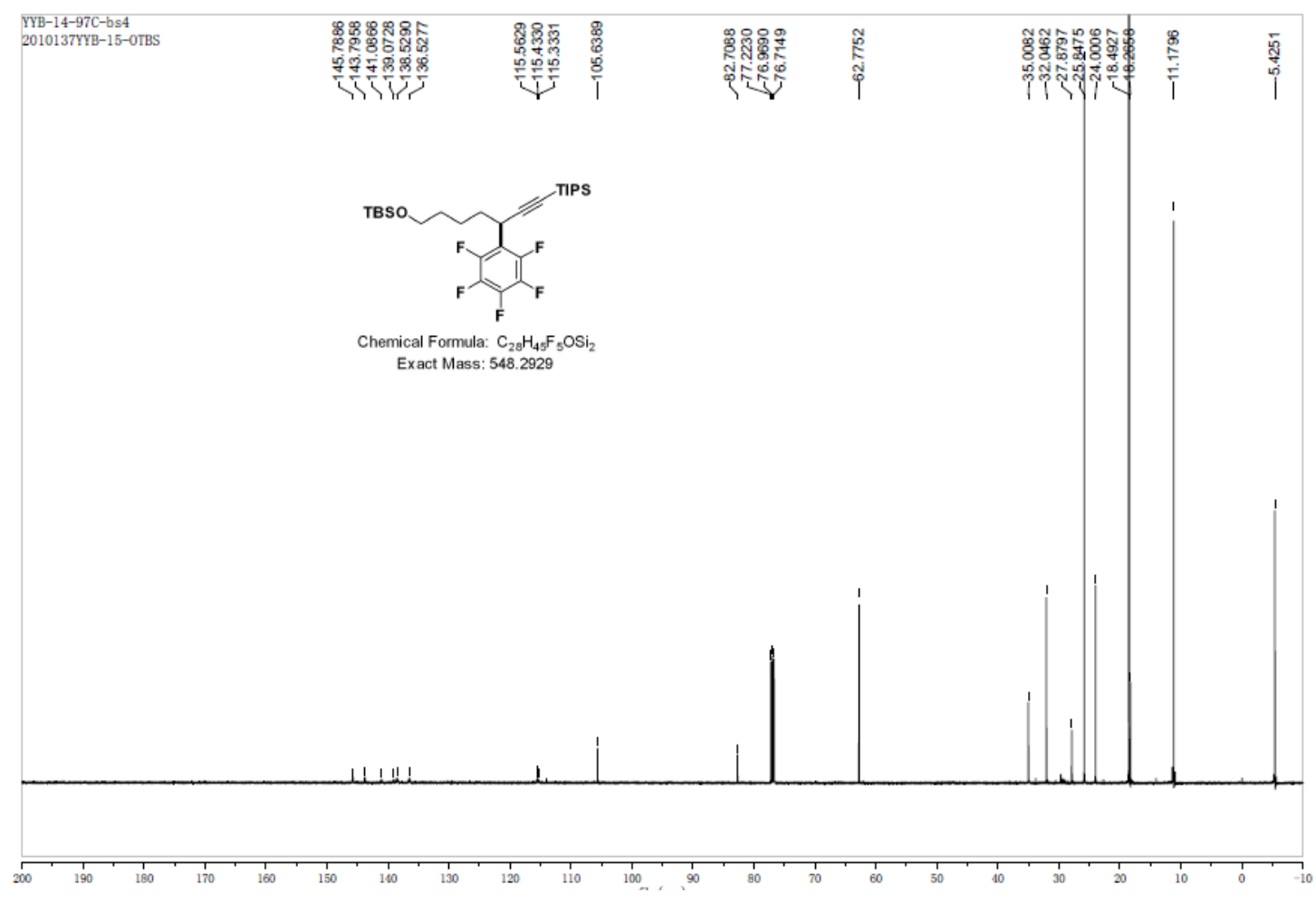

(7-(Benzyloxy)-3-(perfluorophenyl)hept-1-yn-1-yl)triisopropylsilane (3h)

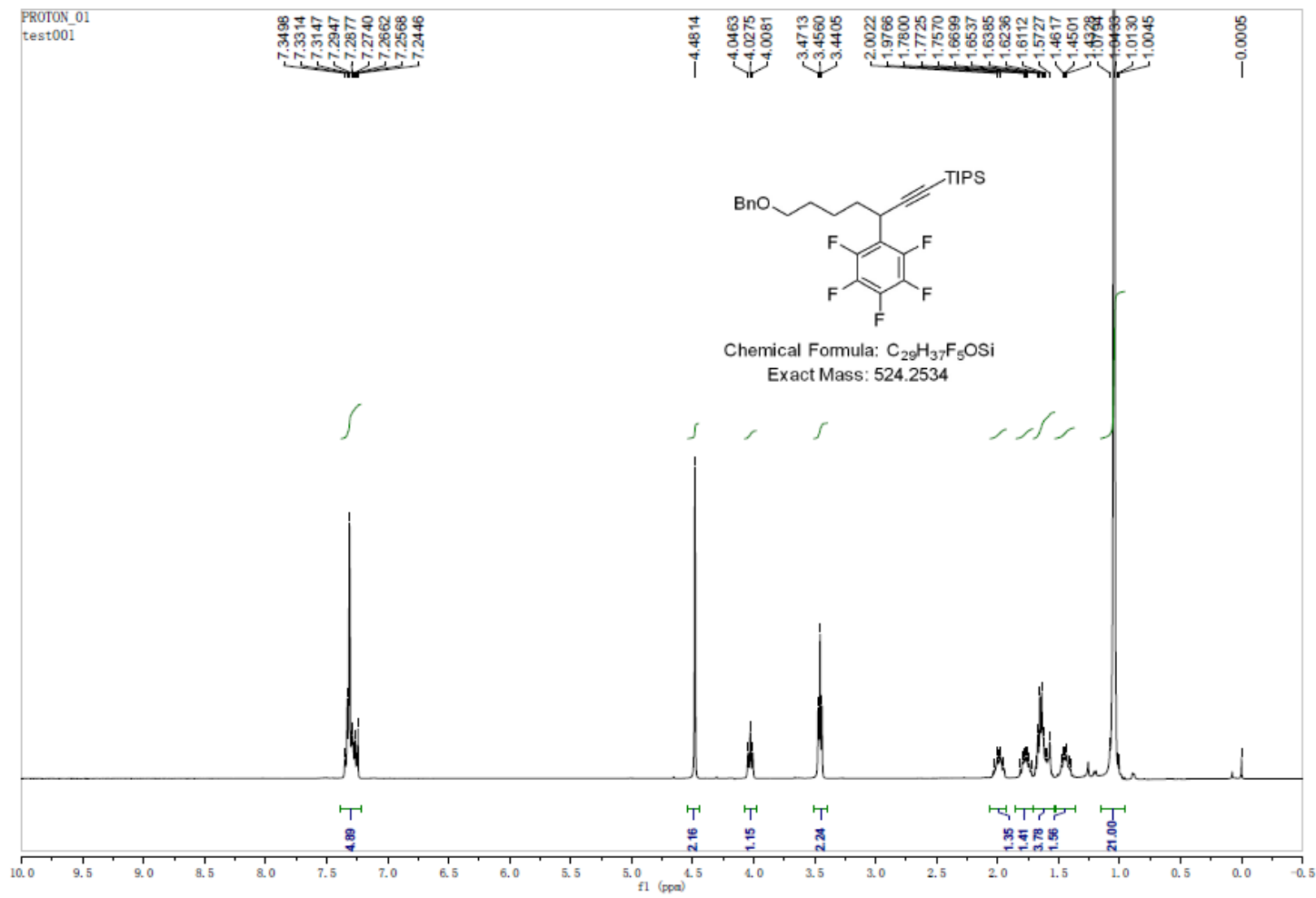



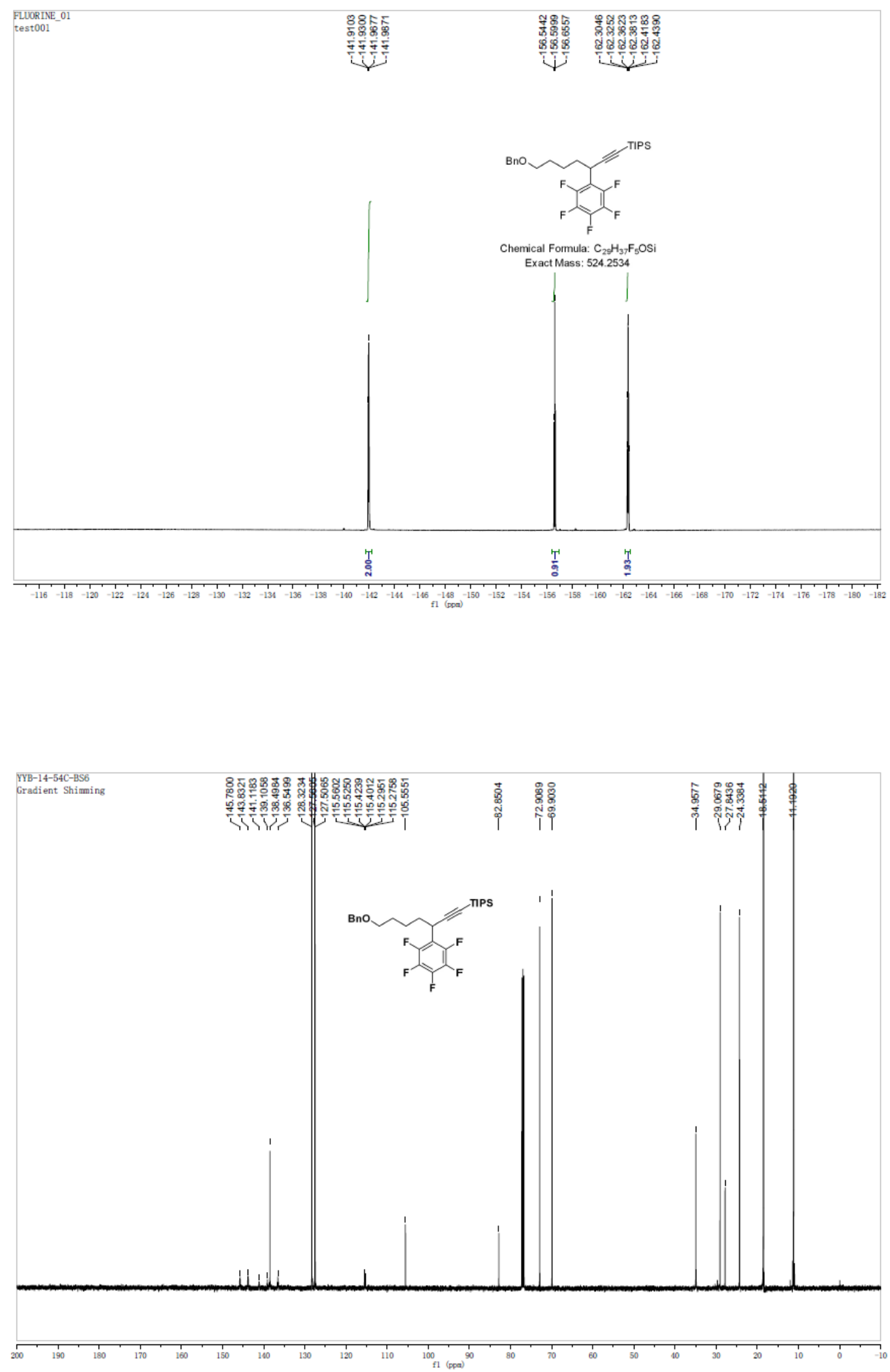
8-Bromo-3-(perfluorophenyl)oct-1-yn-1-yl)triisopropylsilane (3i)
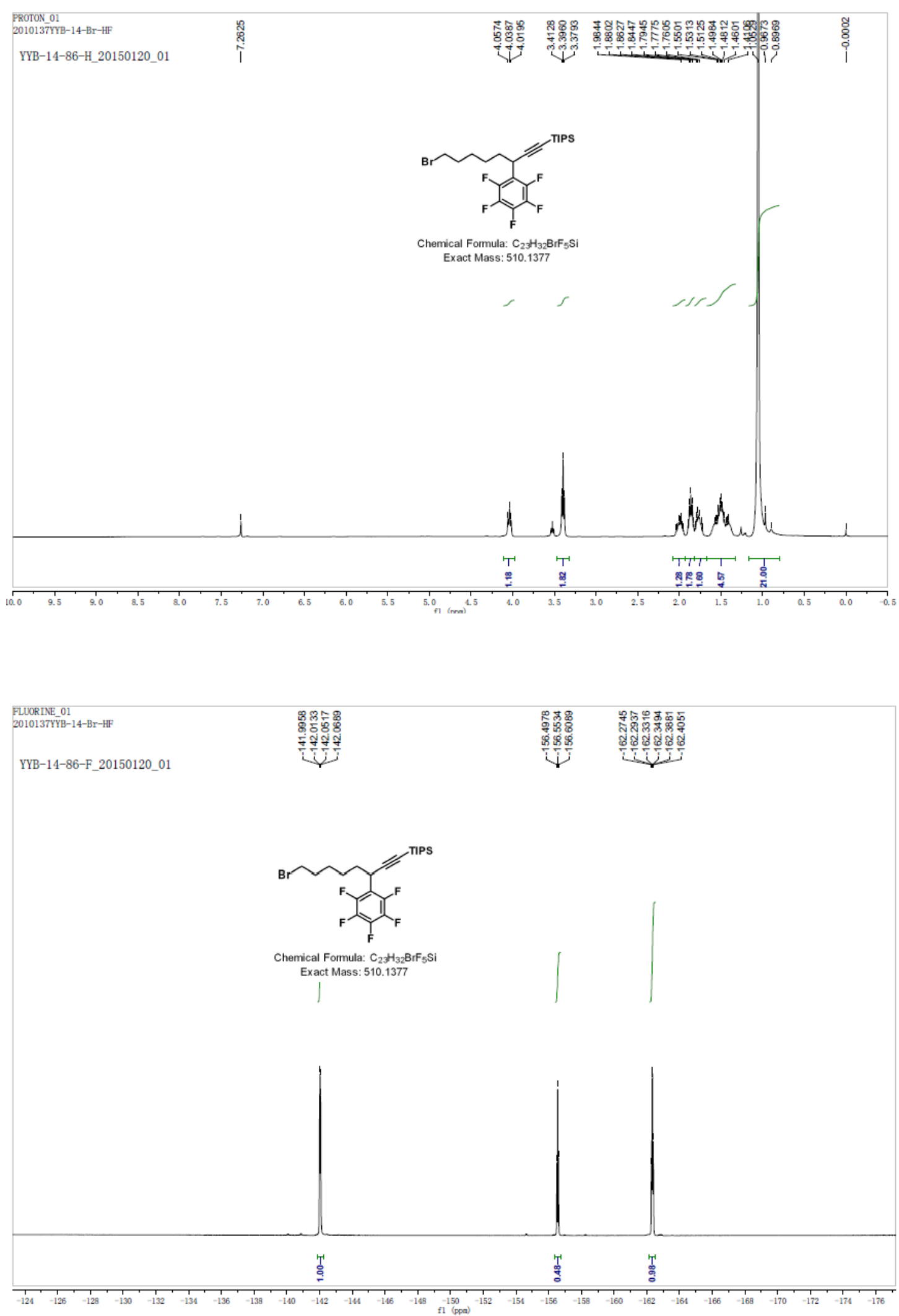


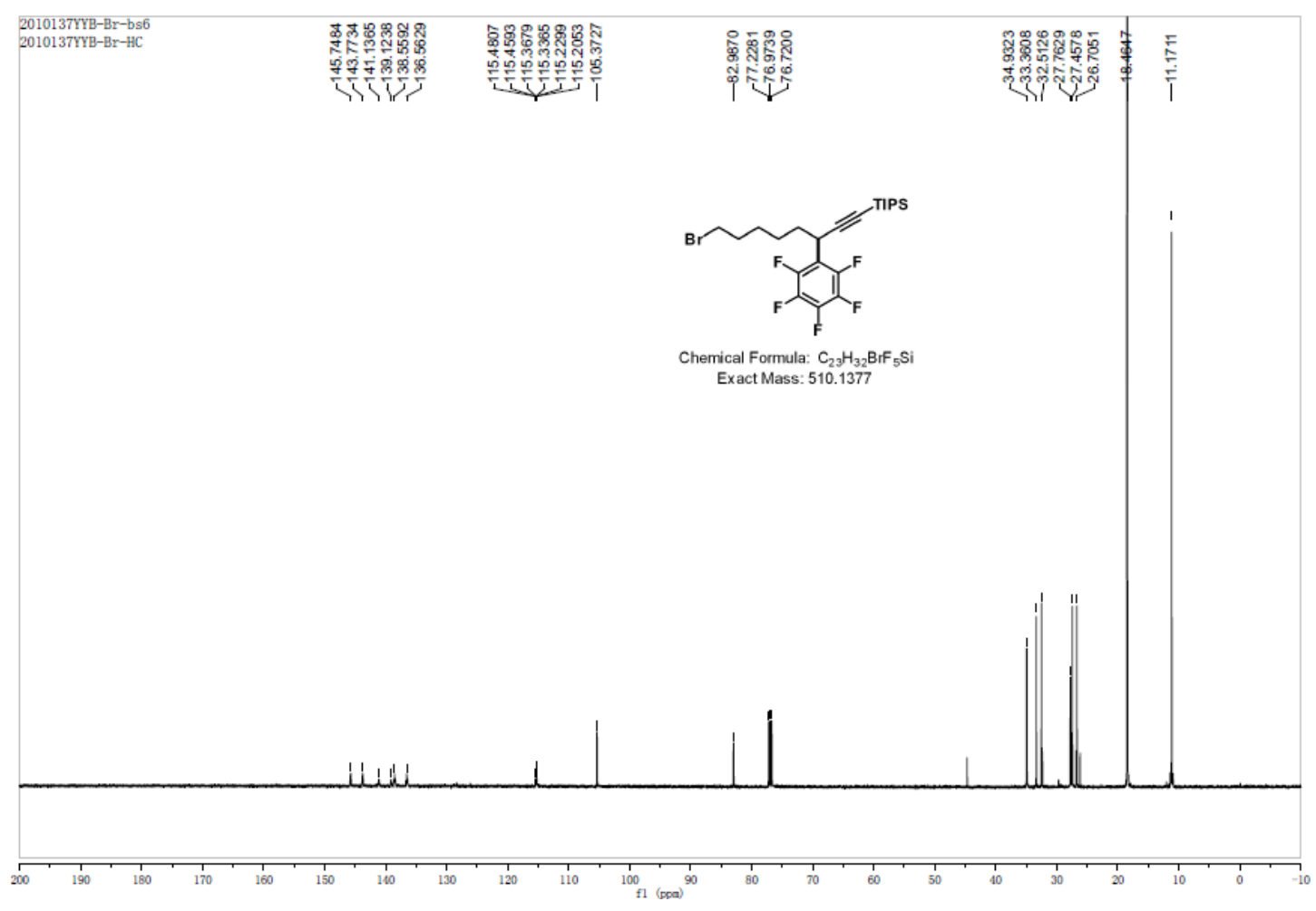

Triethyl(3-(perfluorophenyl)hept-1-yn-1-yl)silane (3j)

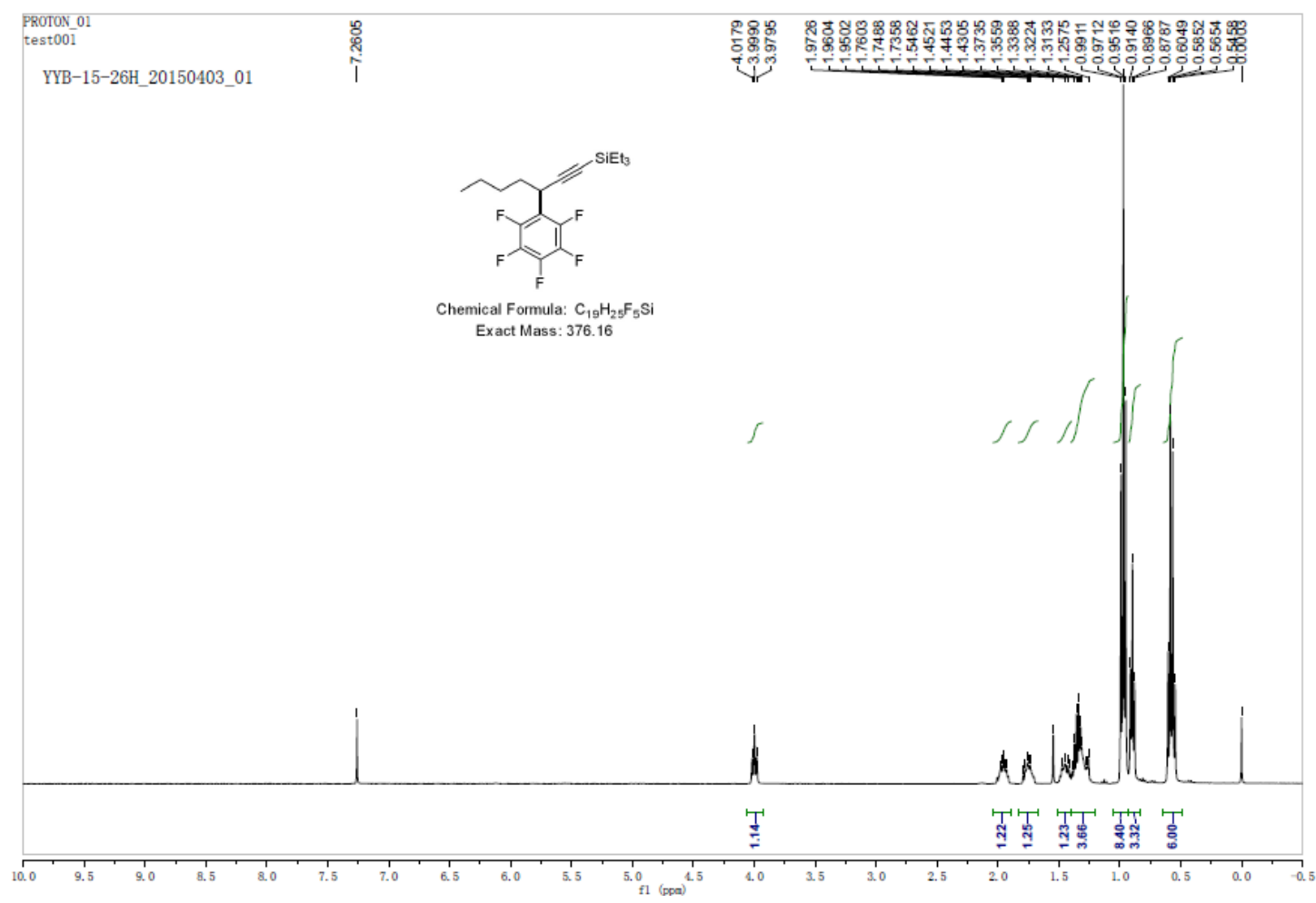



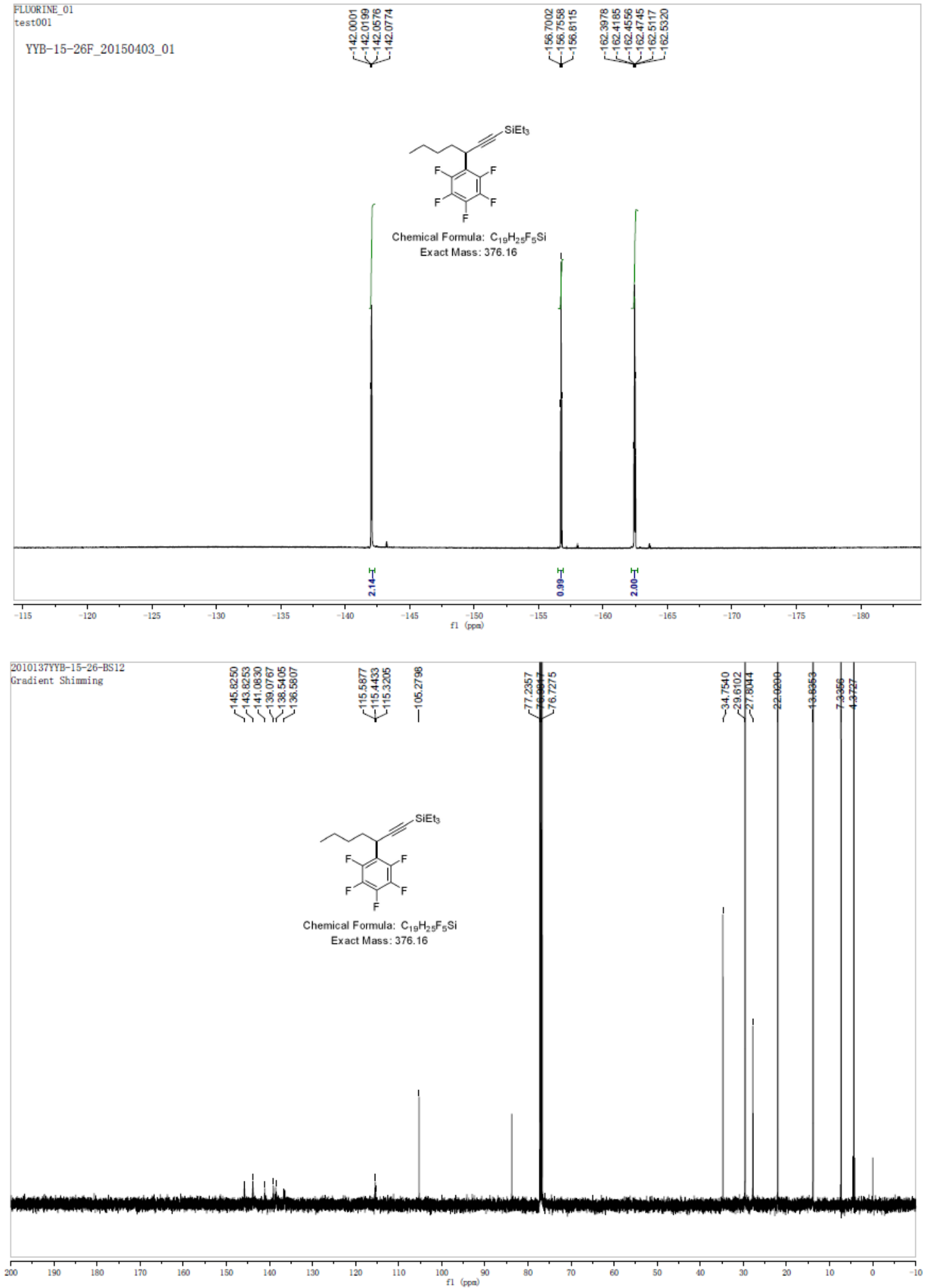
1-(2,2-Dimethylnon-3-yn-5-yl)-2,3,4,5,6-pentafluorobenzene (3k)
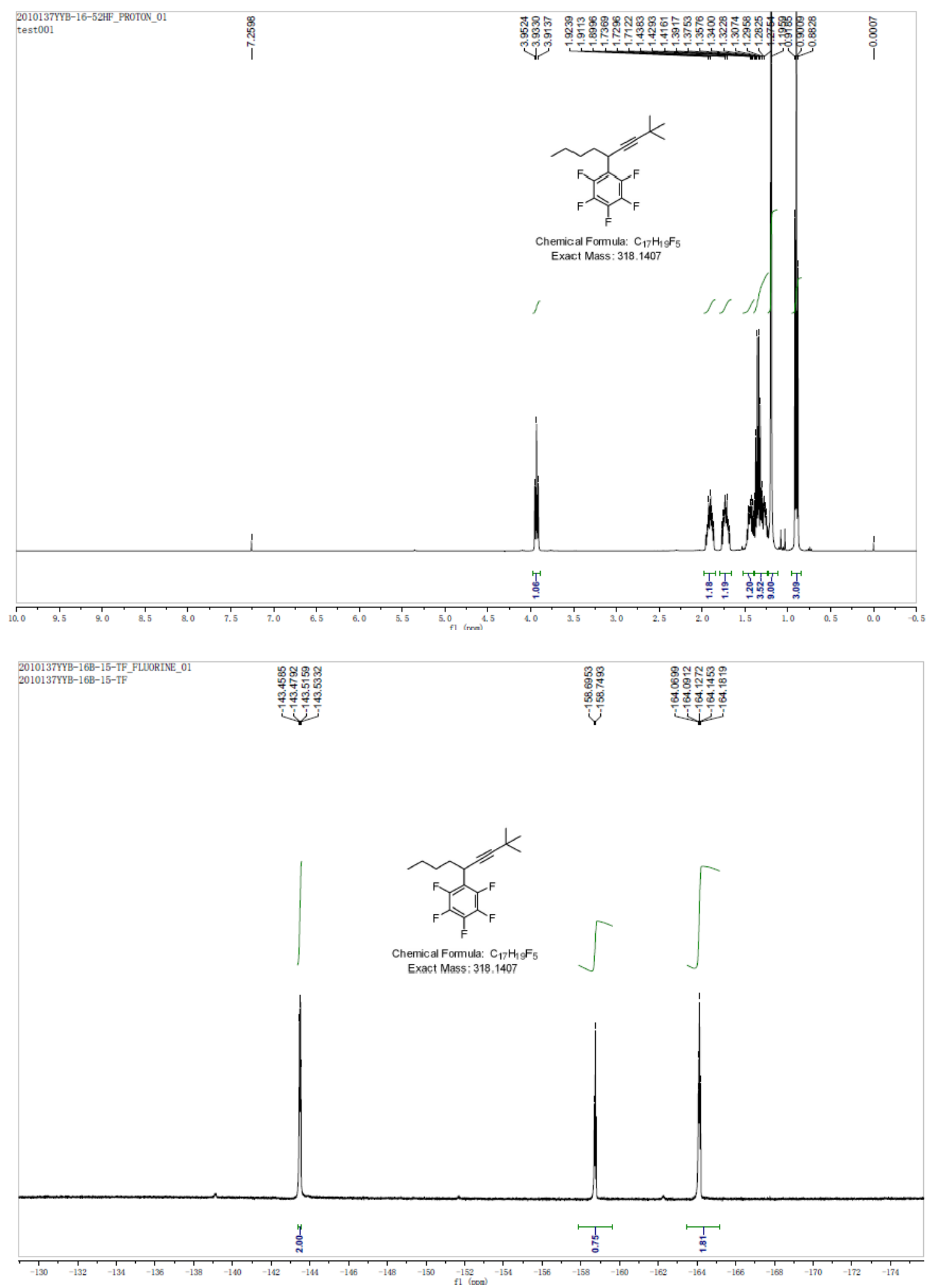


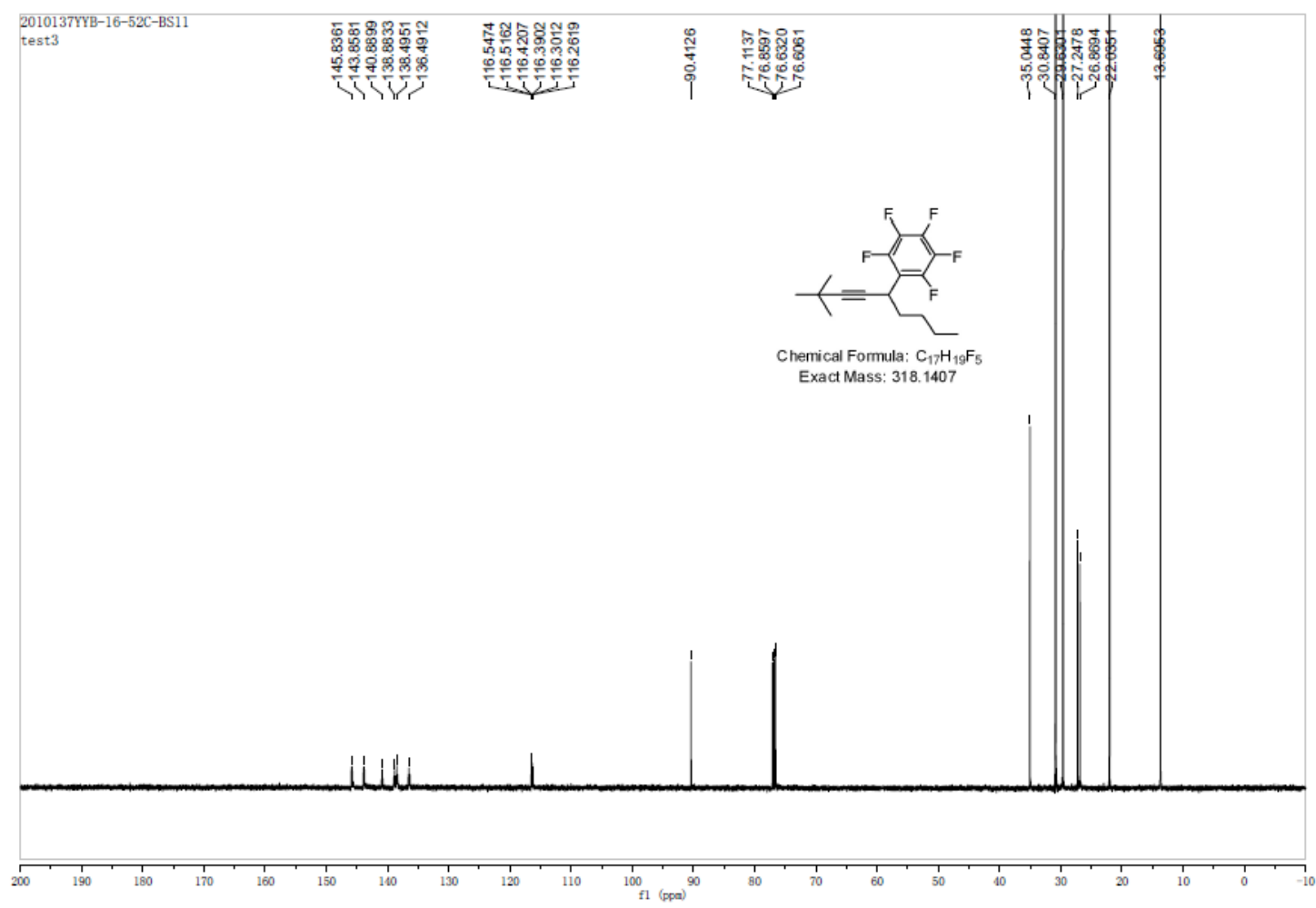

Triisopropyl(3-(2,3,5,6-tetrafluorophenyl)hept-1-yn-1-yl)silane (4a)

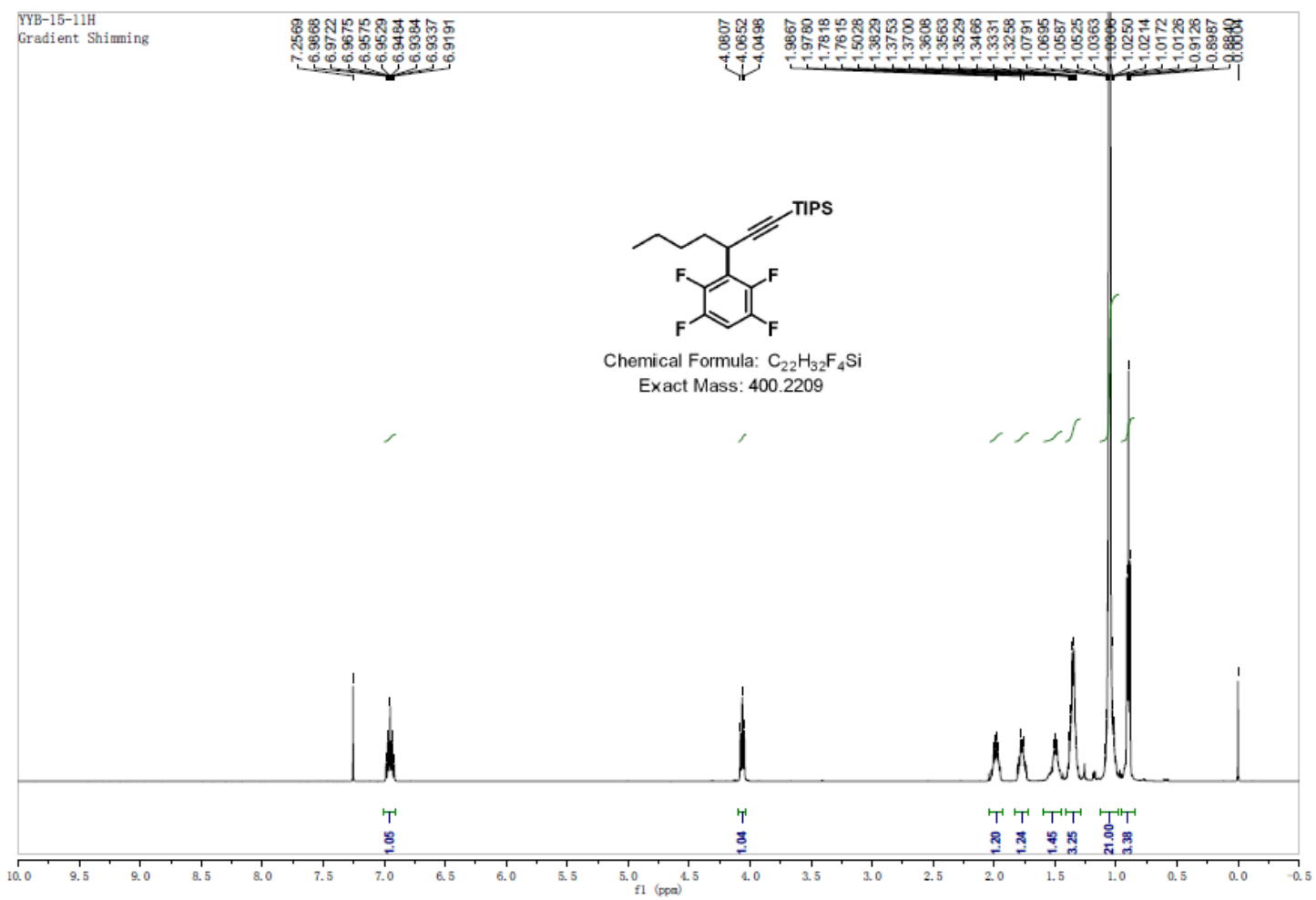



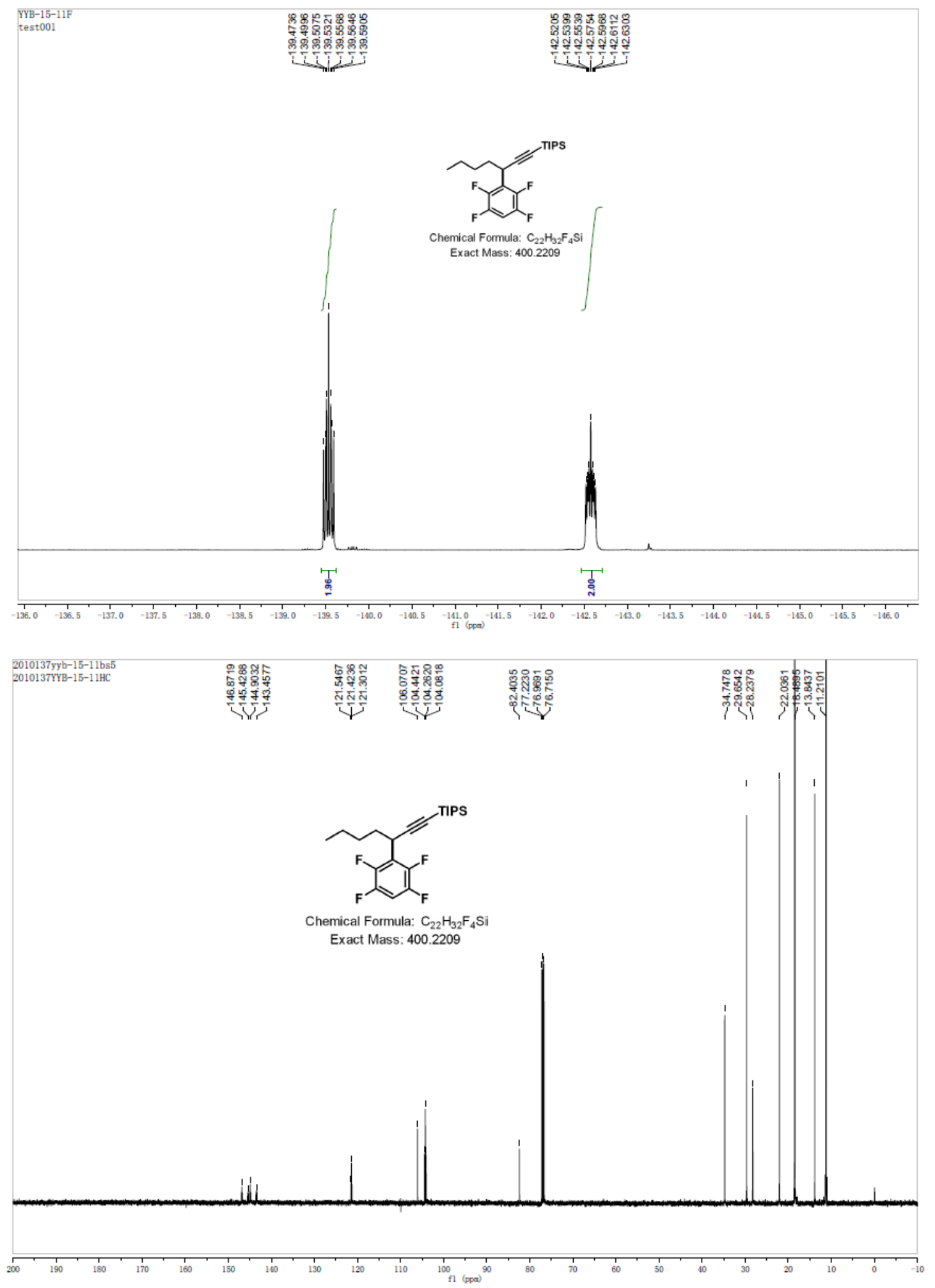
Triethyl(3-(2,3,5,6-tetrafluorophenyl)hept-1-yn-1-yl)silane (4b)
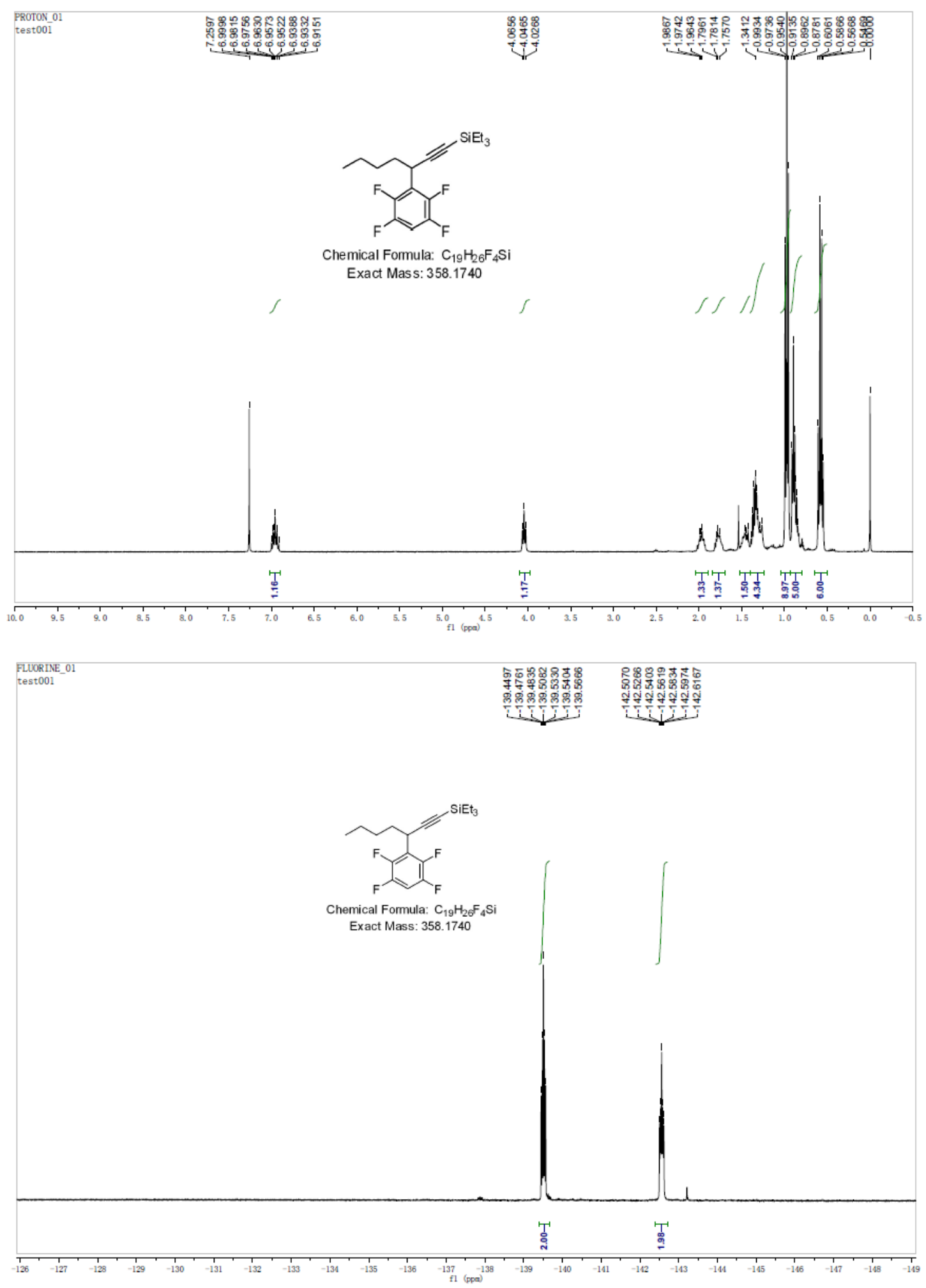


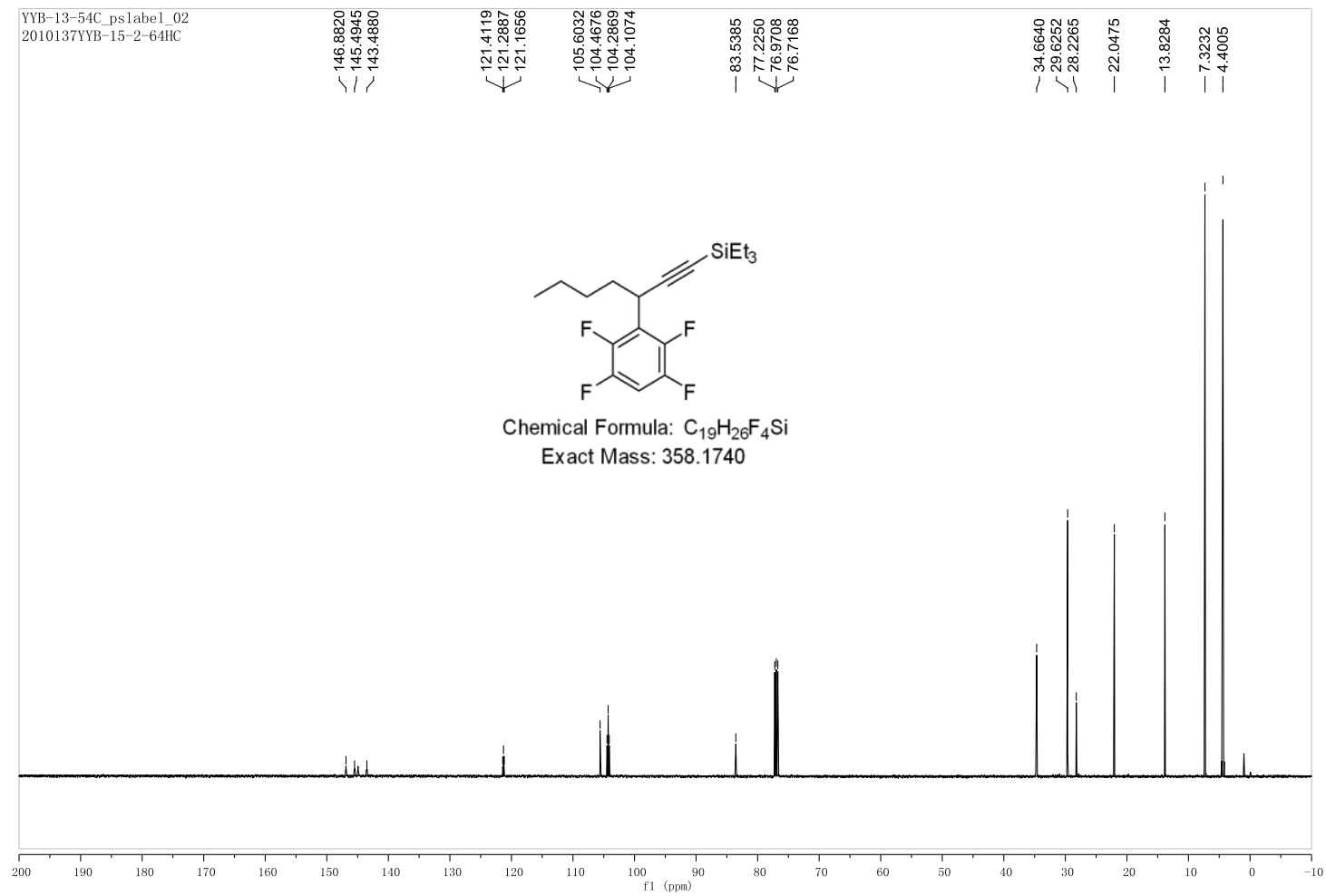

Triisopropyl(5-phenyl-3-(2,3,5,6-tetrafluorophenyl)pent-1-yn-1-yl)silane (4c)

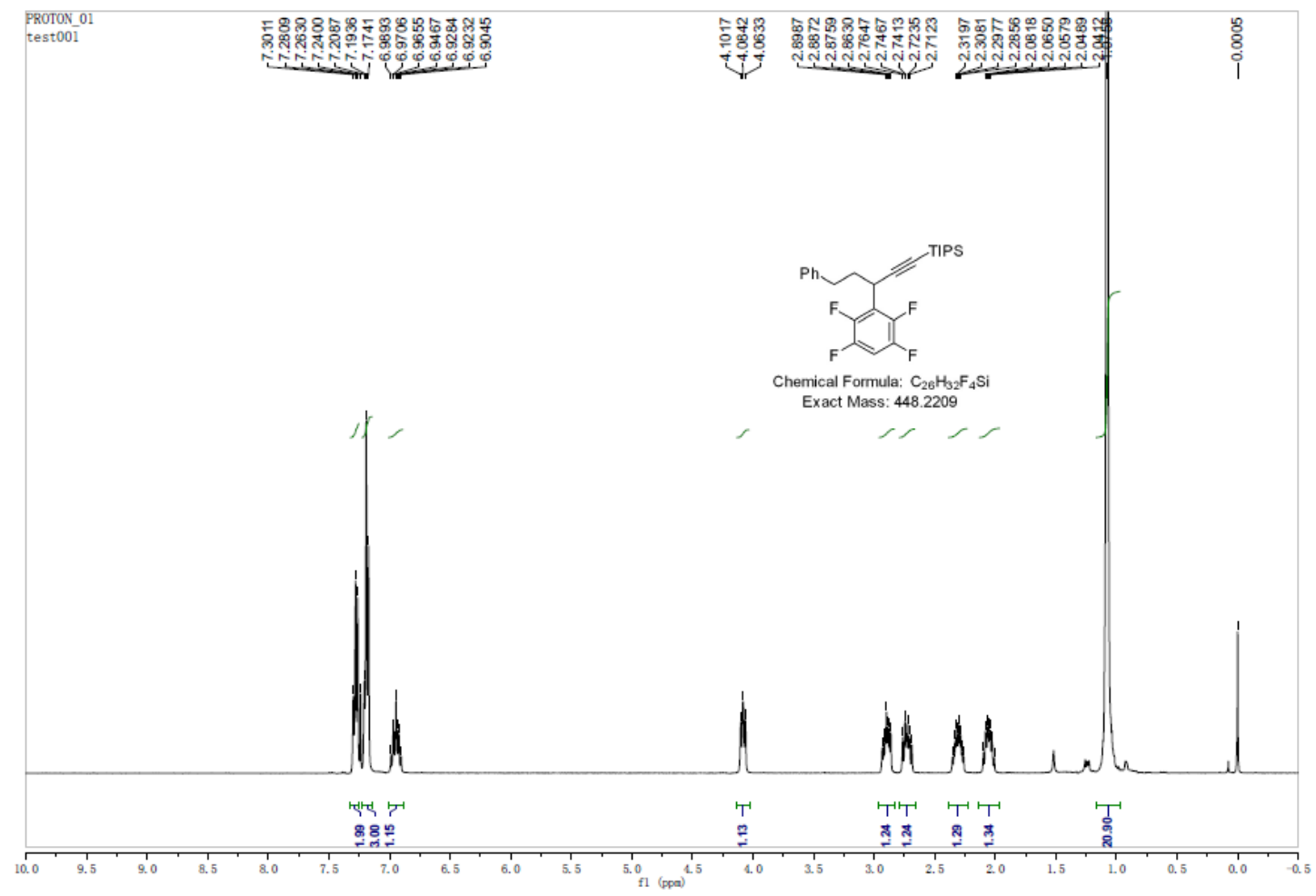



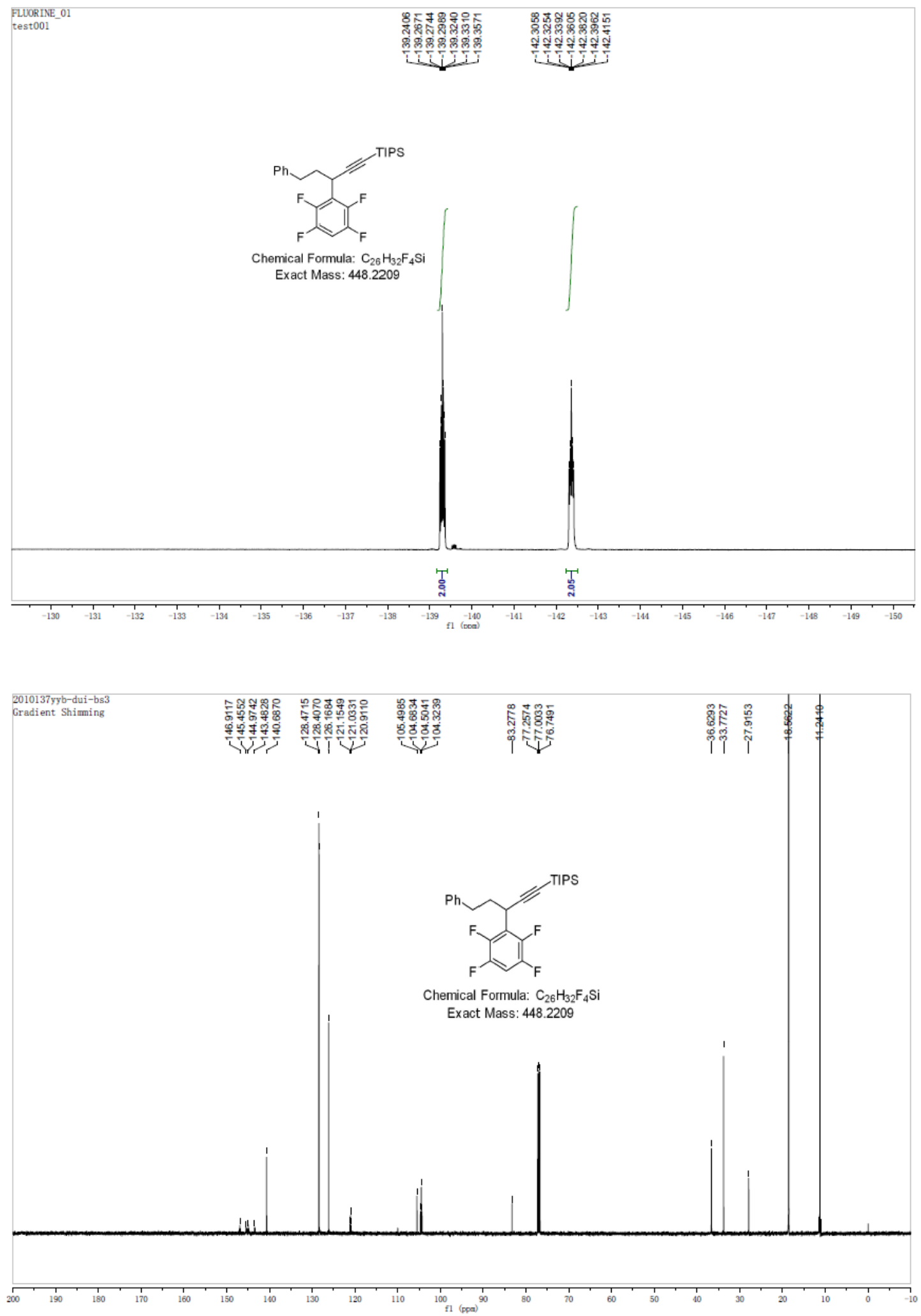
8-Bromo-3-(2,3,5,6-tetrafluorophenyl)oct-1-yn-1-yl)triisopropylsilane

(4d)
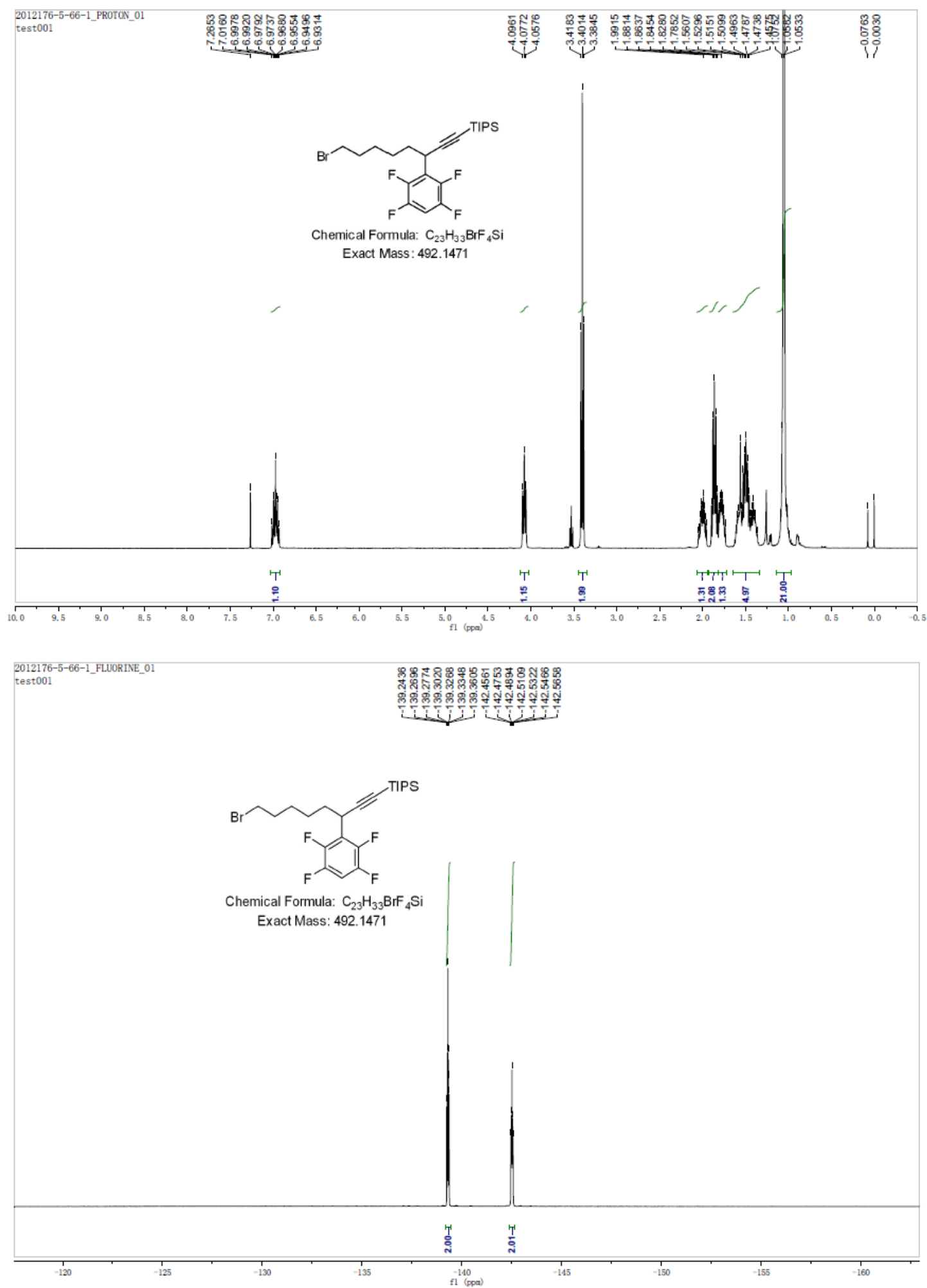


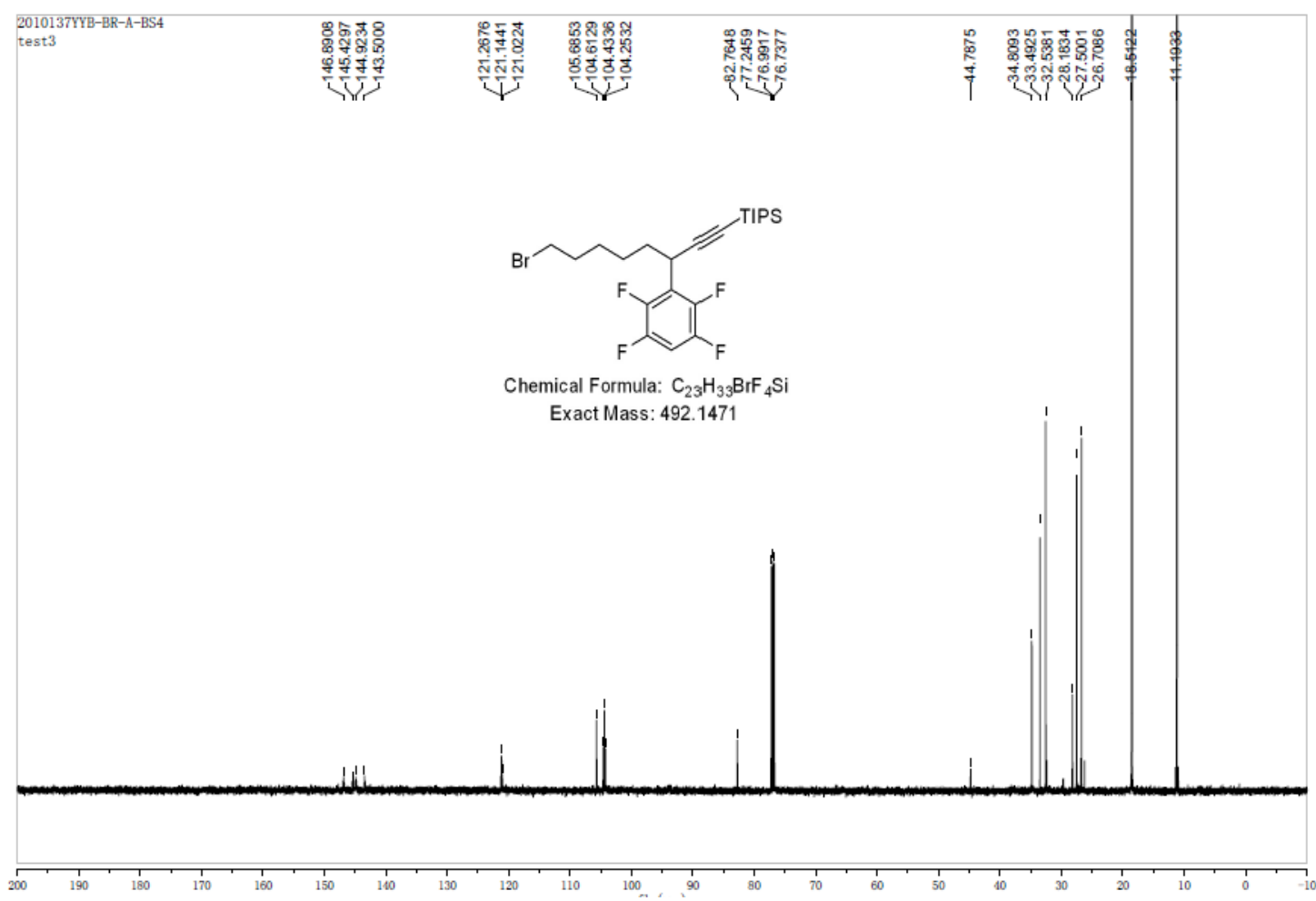

Triisopropyl(3-(2,3,4,6-tetrafluorophenyl)hept-1-yn-1-yl)silane (4e)

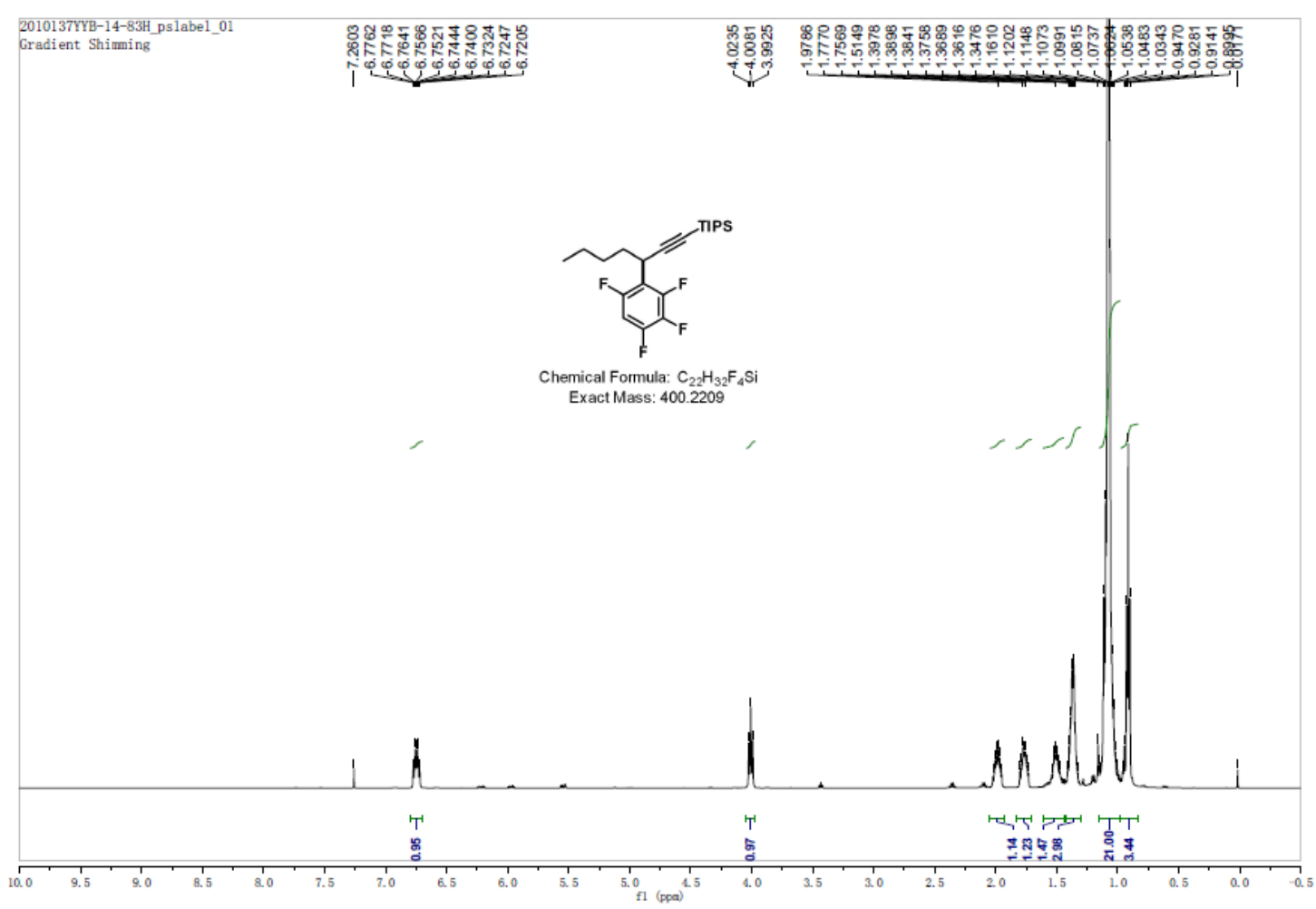



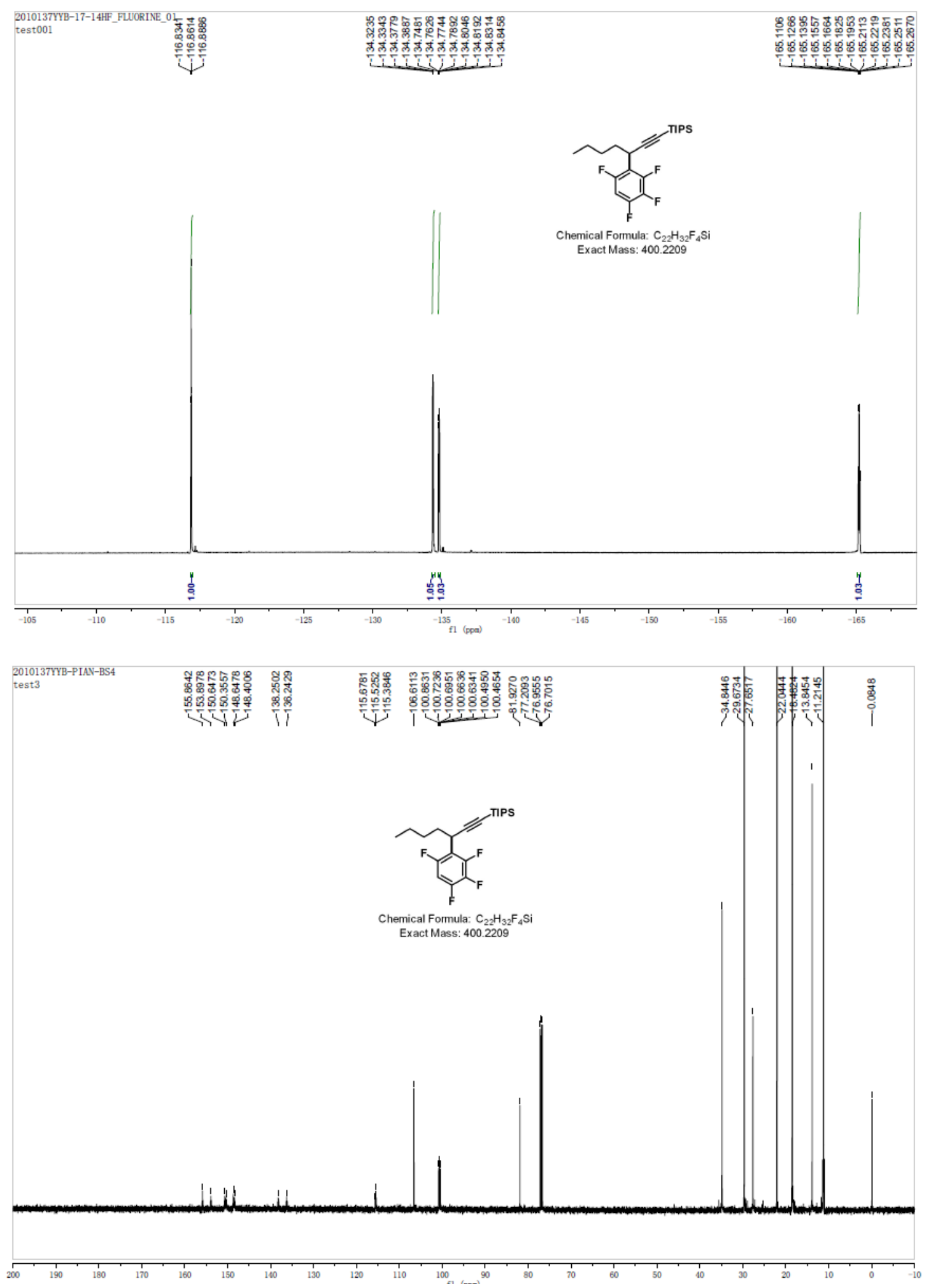
Triisopropyl(5-phenyl-3-(2,3,4,6-tetrafluorophenyl)pent-1-yn-1-yl)silane (4f)
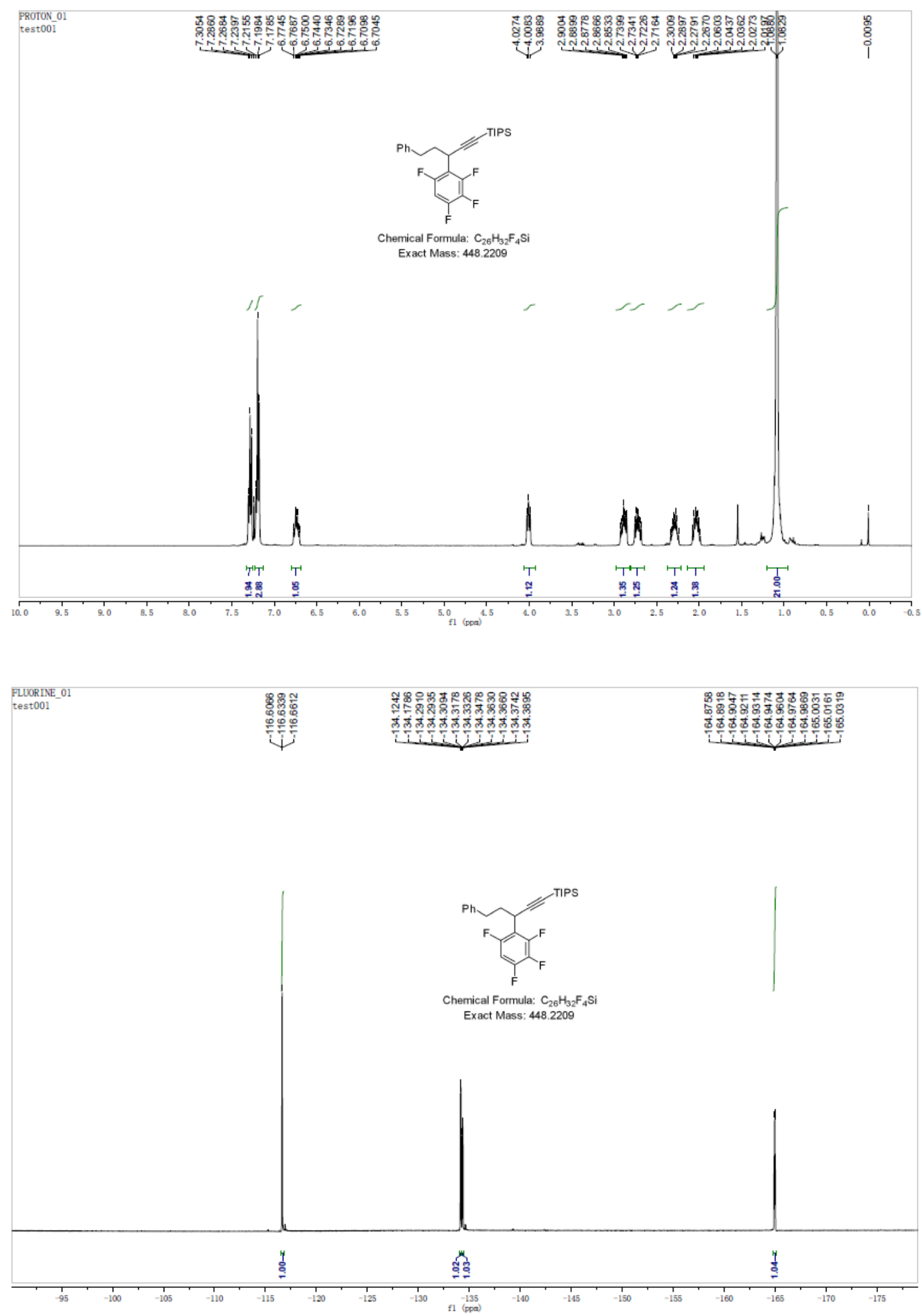


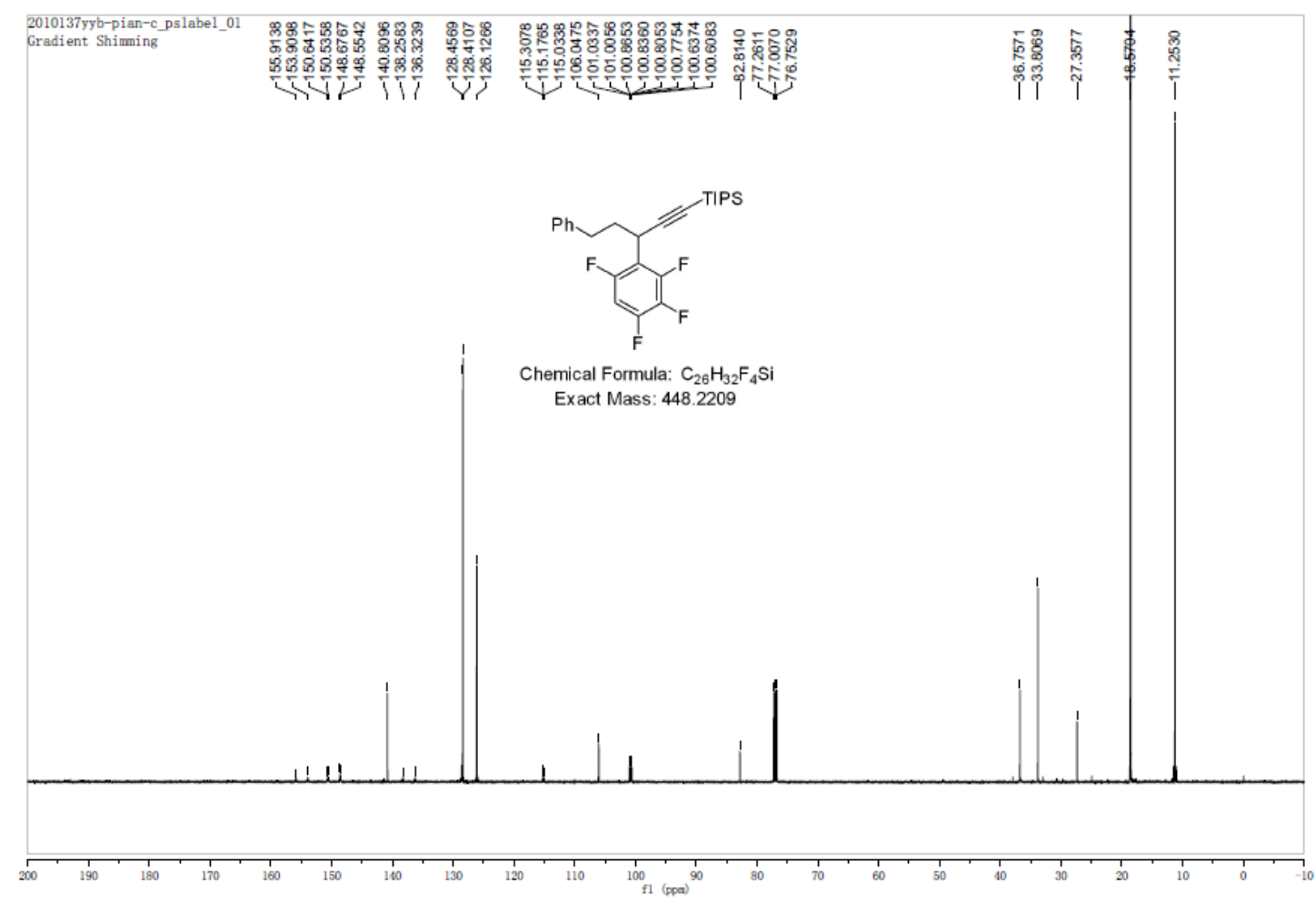

7-(Benzyloxy)-3-(2,3,4,6-tetrafluorophenyl)hept-1-yn-1-yl)triisopropylsilane (4g)

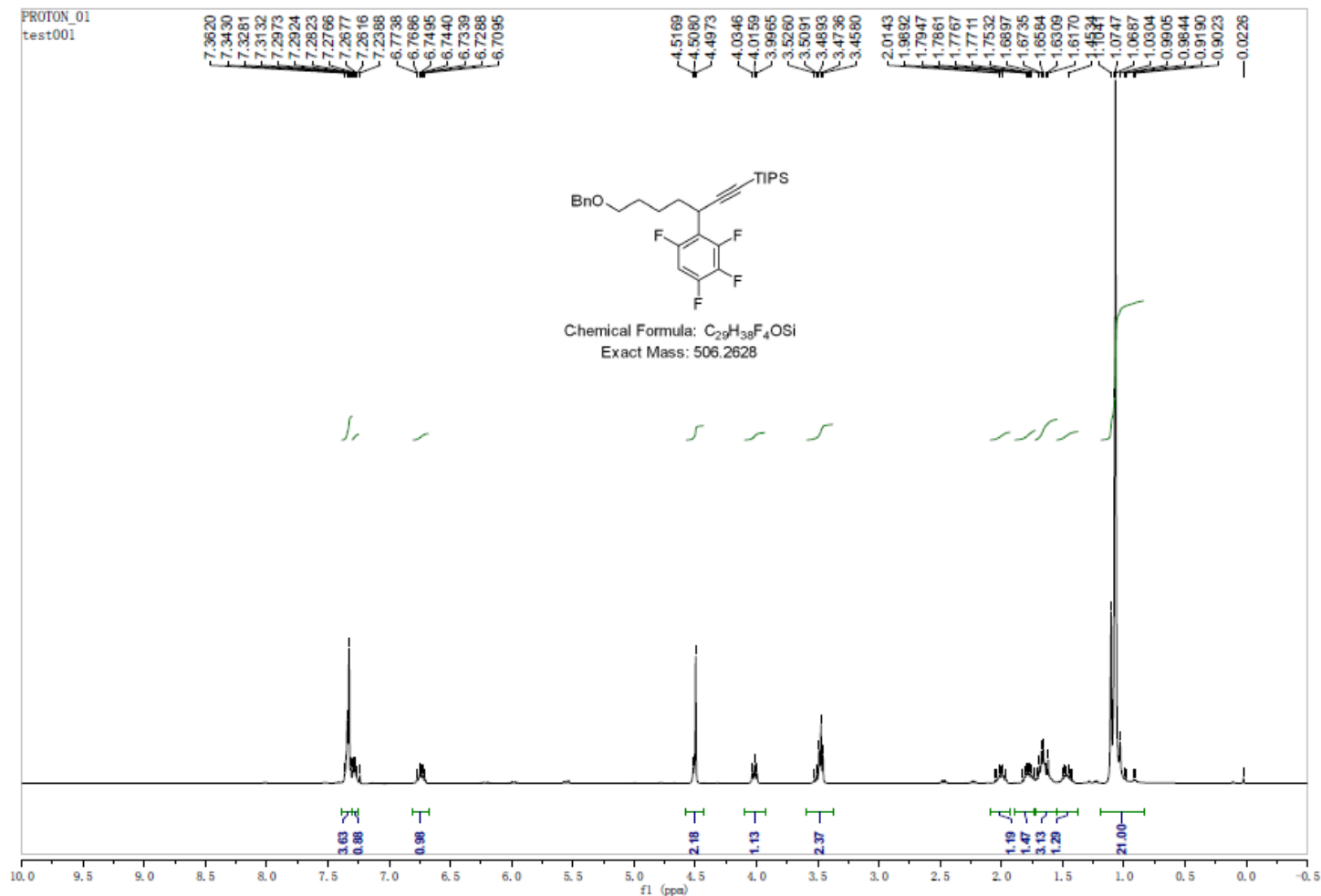



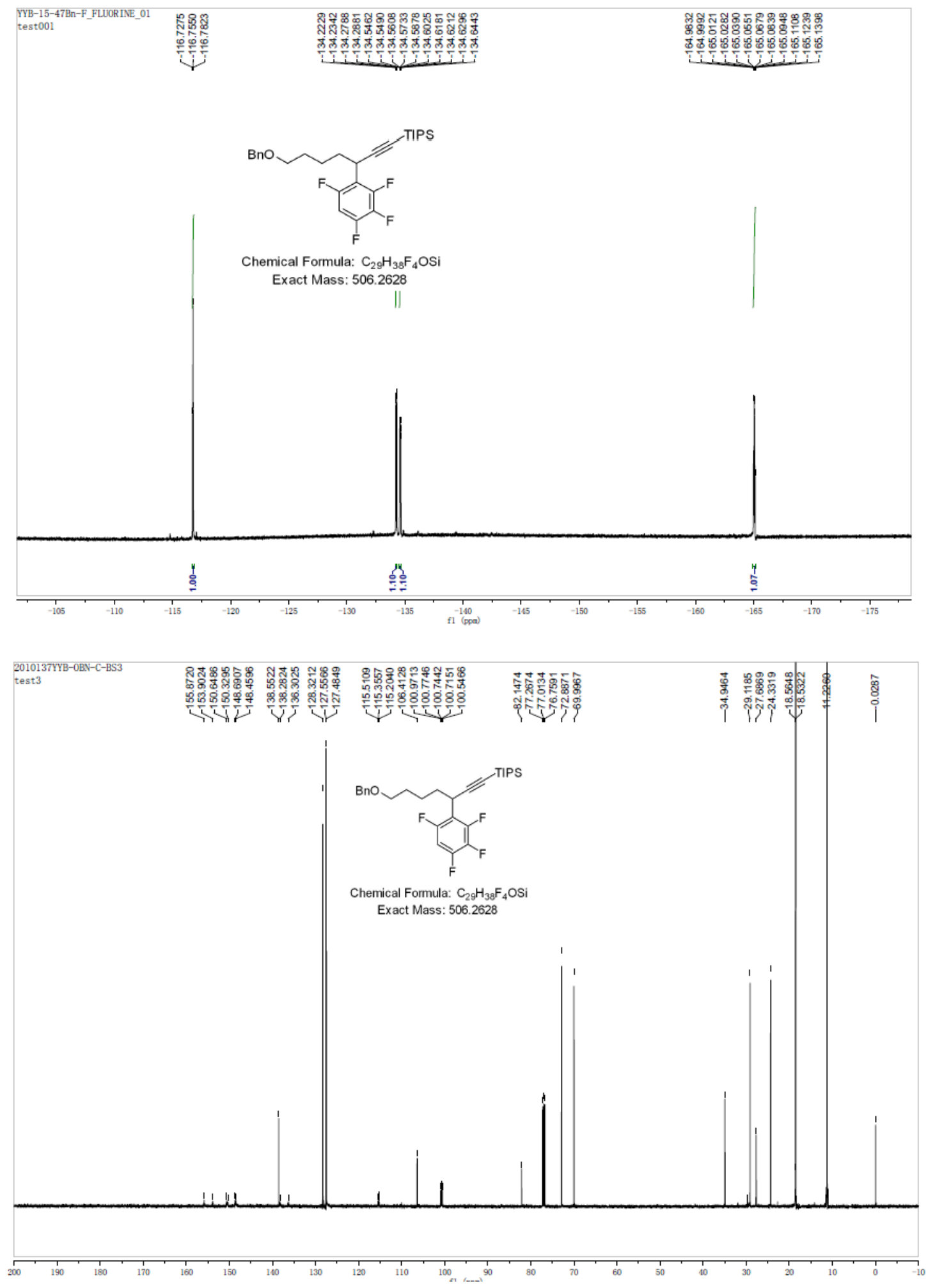
Triisopropyl(3-(2,3,5,6-tetrafluoro-4'-methoxy-[1,1'-biphenyl]-4-yl)hept-1-yn-1-yl)silane (4h)
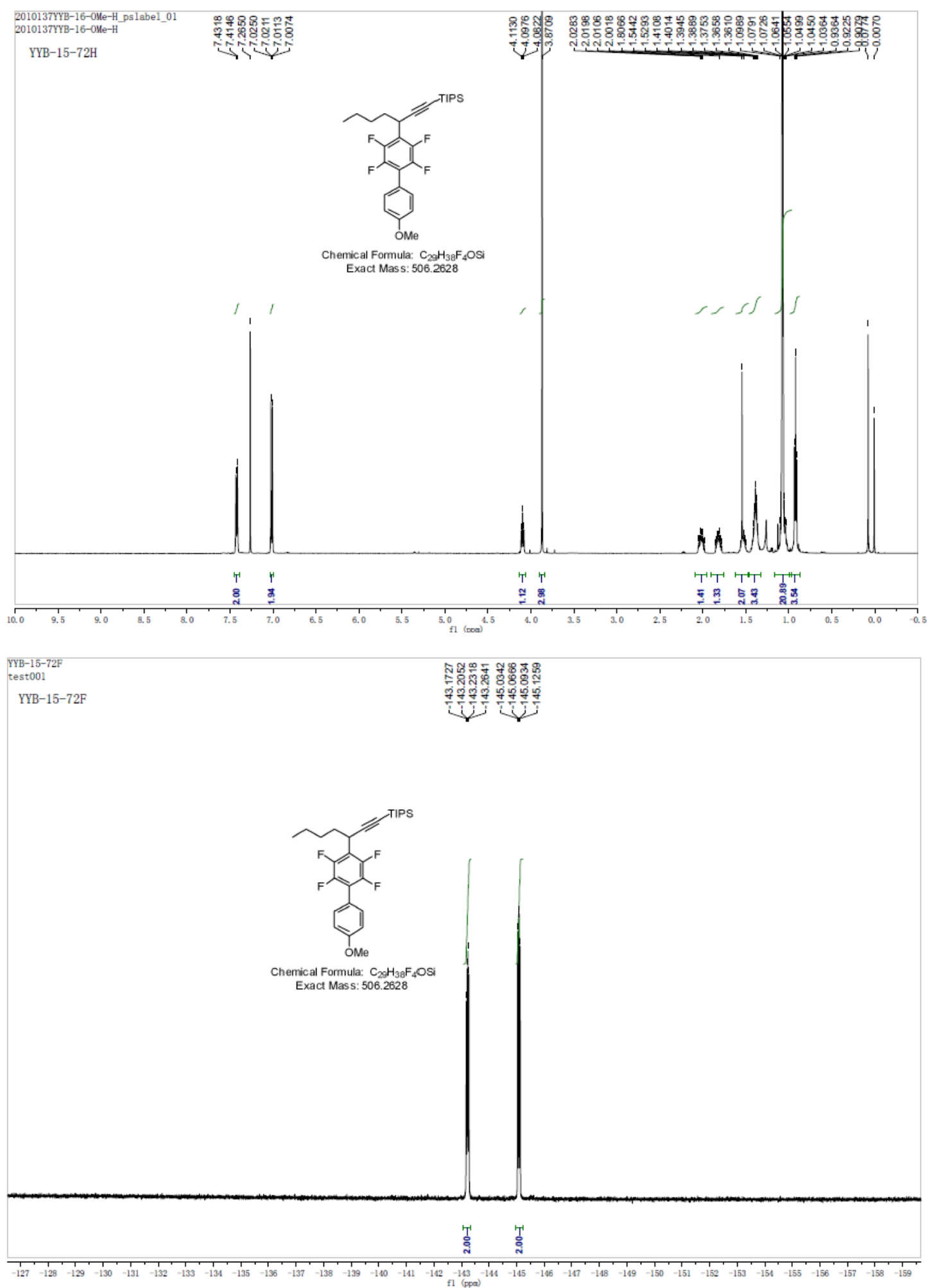


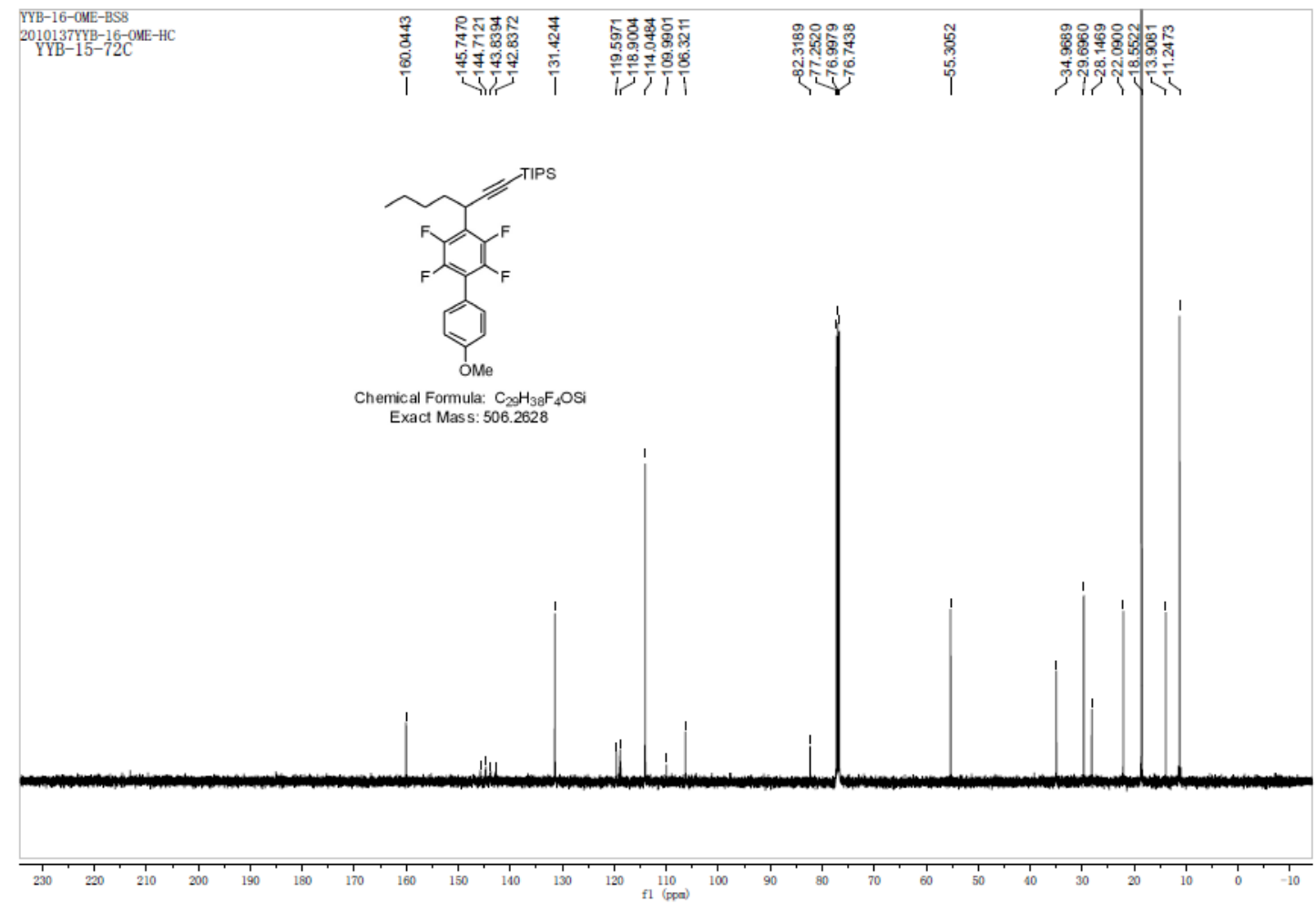

(3-(4'-Bromo-2,3,5,6-tetrafluoro-[1,1'-biphenyl]-4-yl)-5-methylhex-1-yn-1-yl)triisopropylsilane (4i)

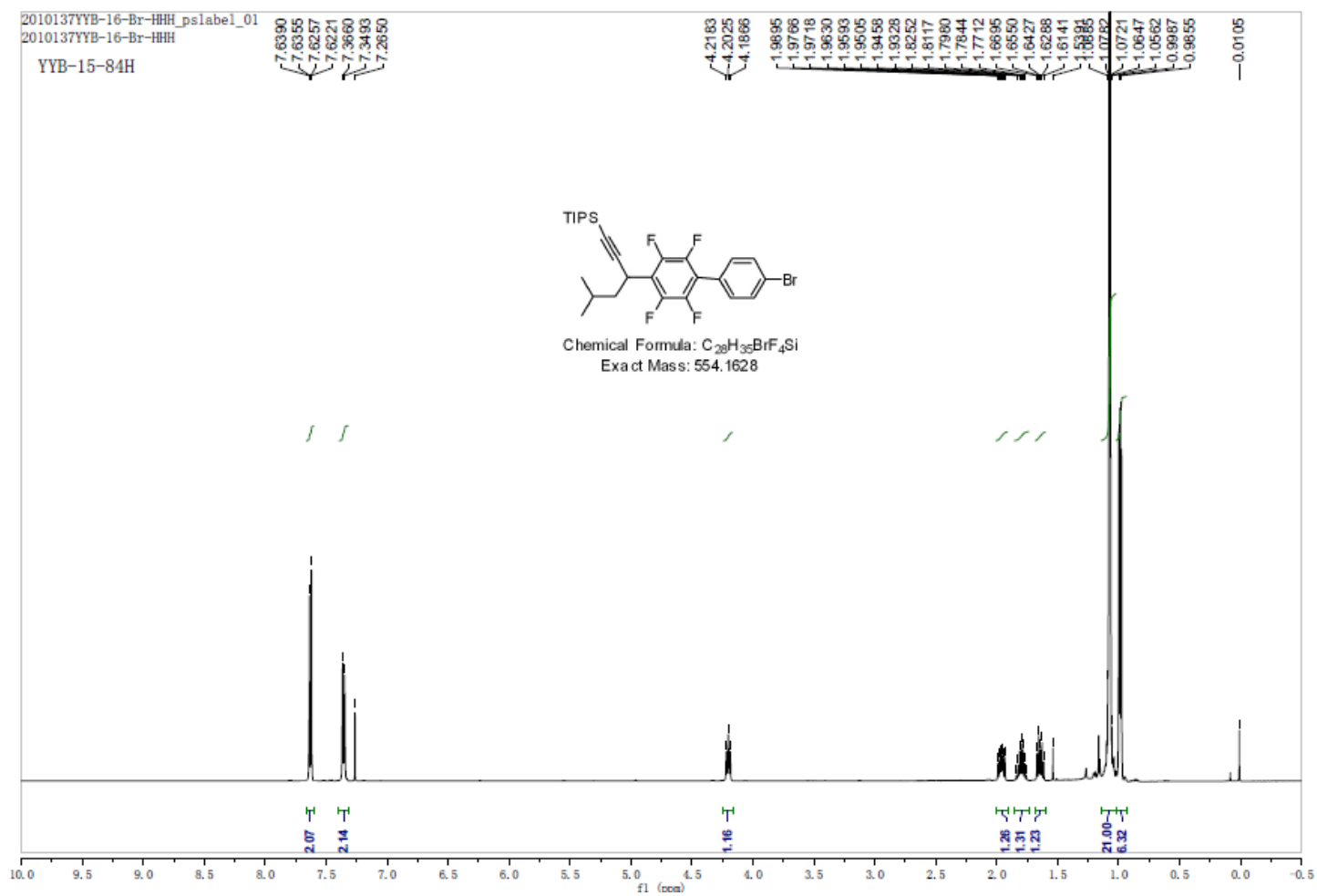



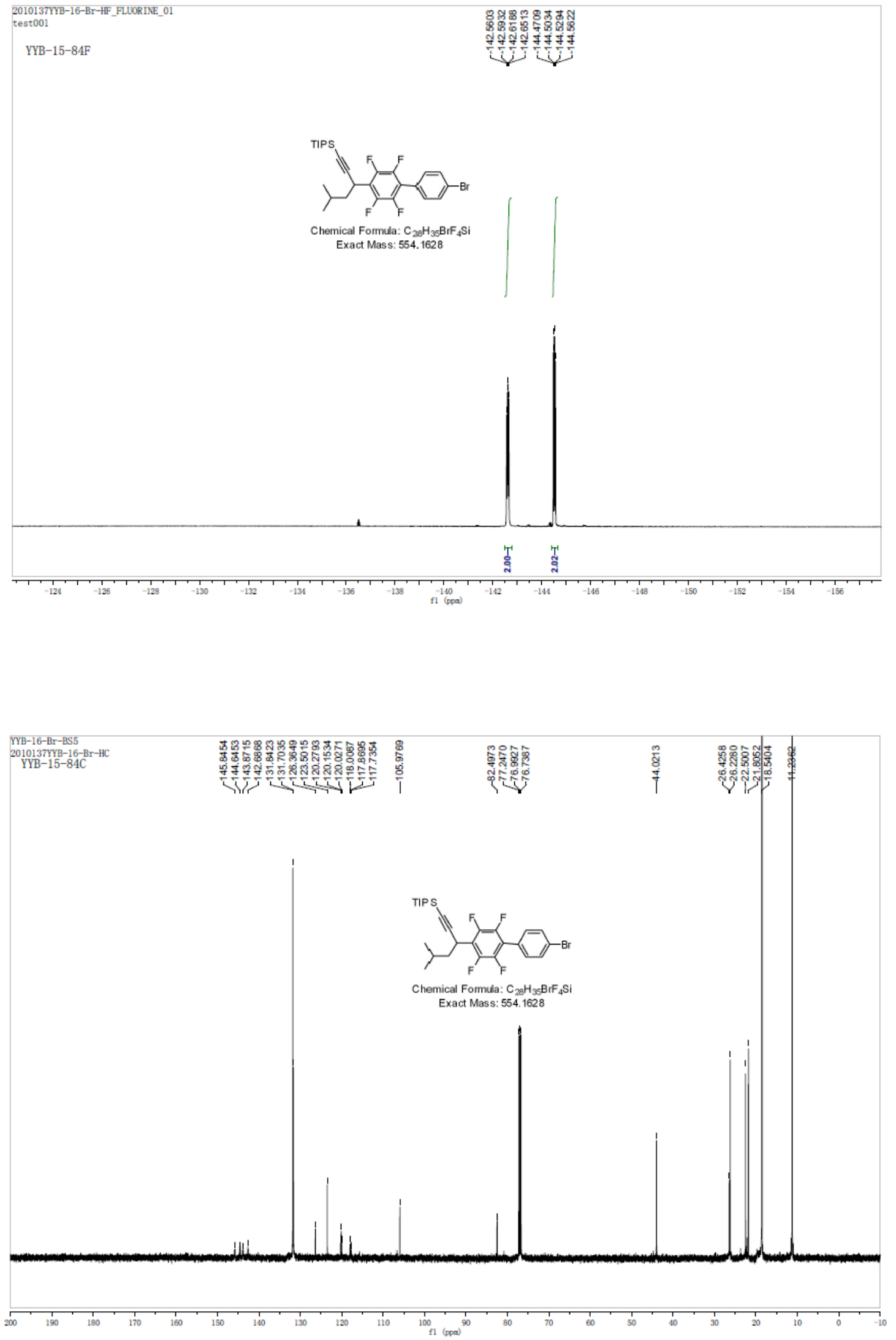
2-(2,3,5,6-Tetrafluoro-4-(1-(triisopropylsilyl)hept-1-yn-3-yl)phenyl)pyridine (4j)

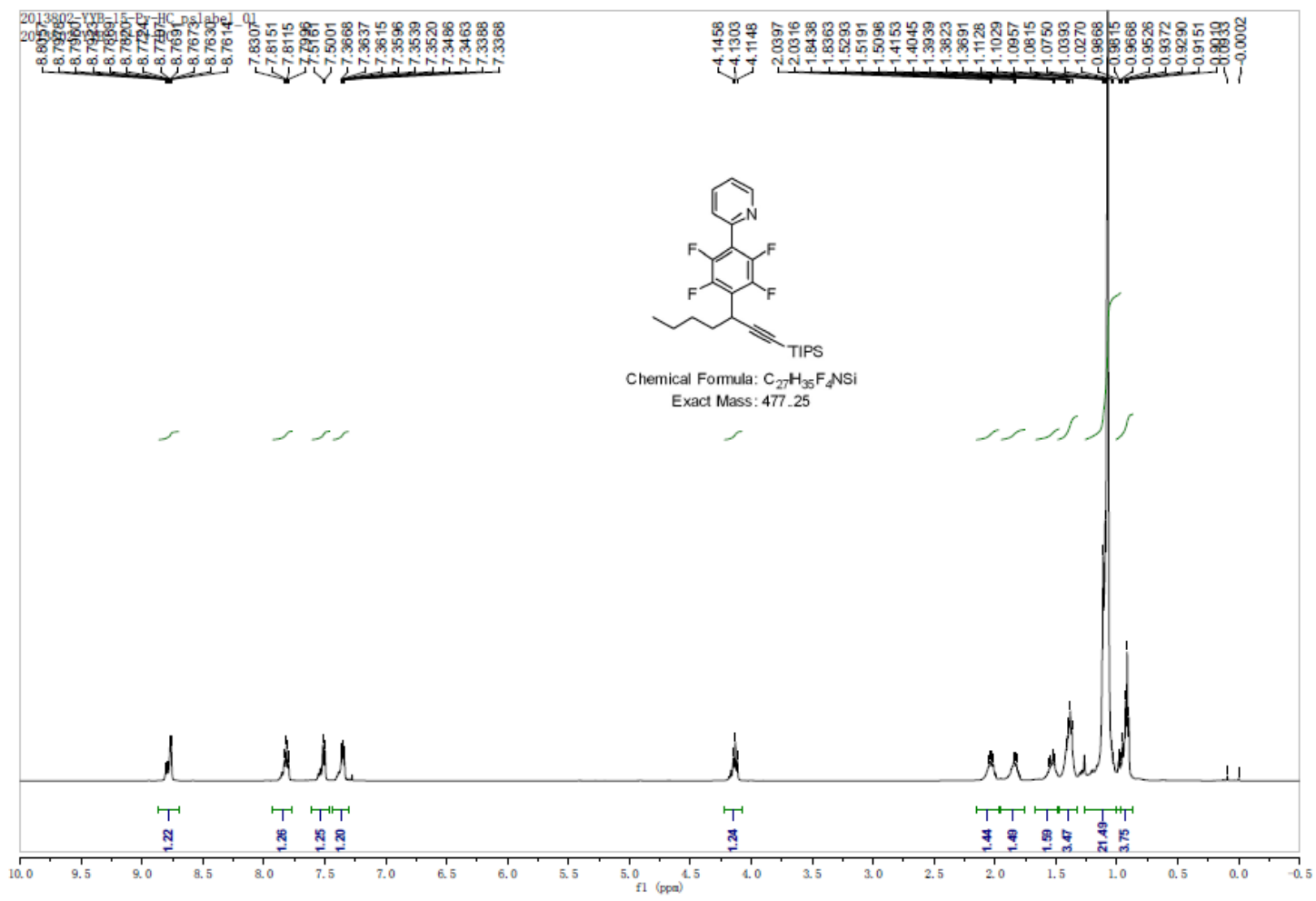

\begin{tabular}{|c|c|}
\hline $\begin{array}{l}\text { 2013802-YYB-15-PY-HF_FLORINE_01 } \\
\text { test001 }\end{array}$ & 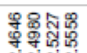 \\
\hline YYB-15-70F & $\frac{7}{7} \frac{7}{5} \frac{7}{5}$ \\
\hline
\end{tabular}

YYB-15-70F

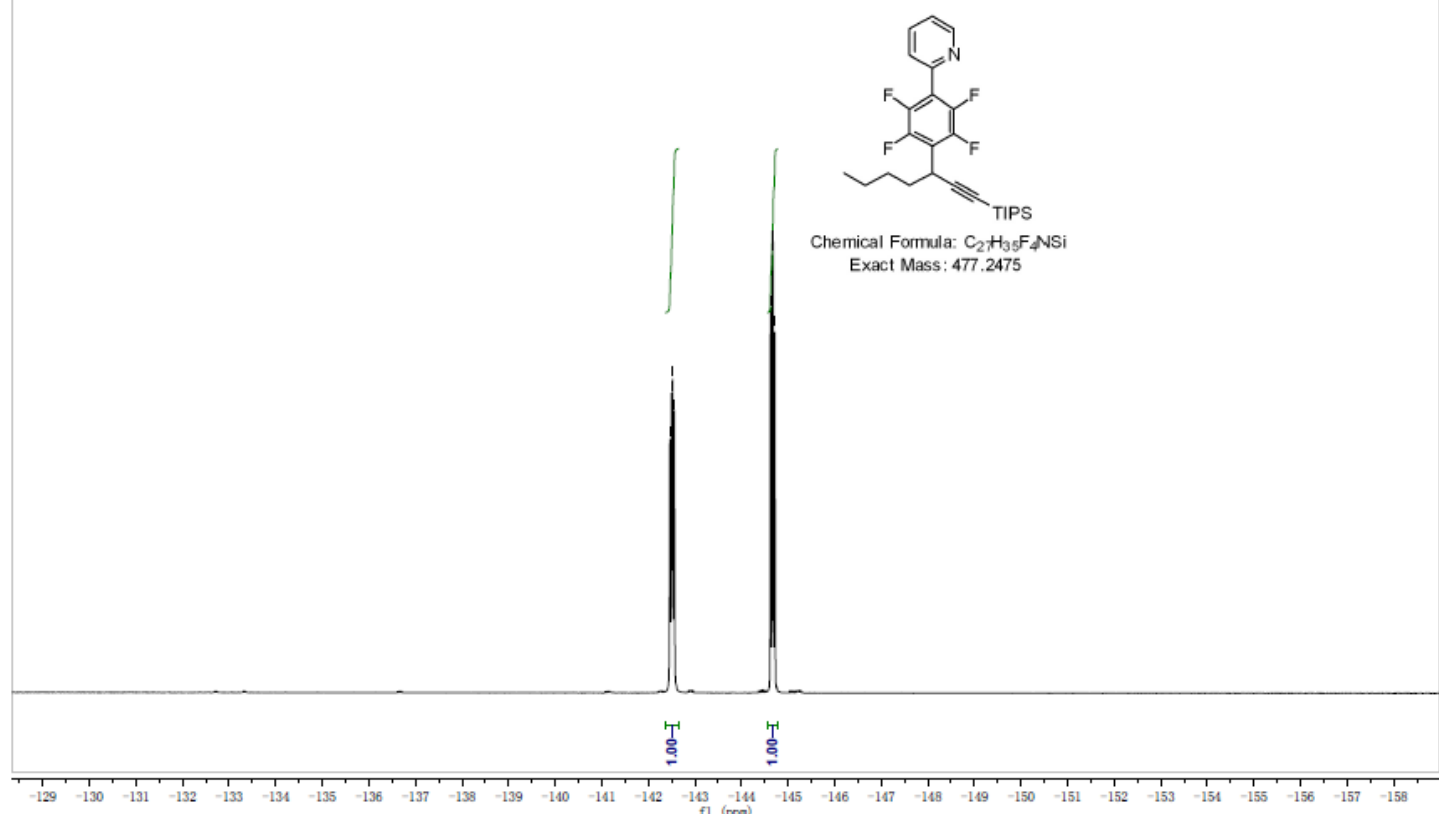




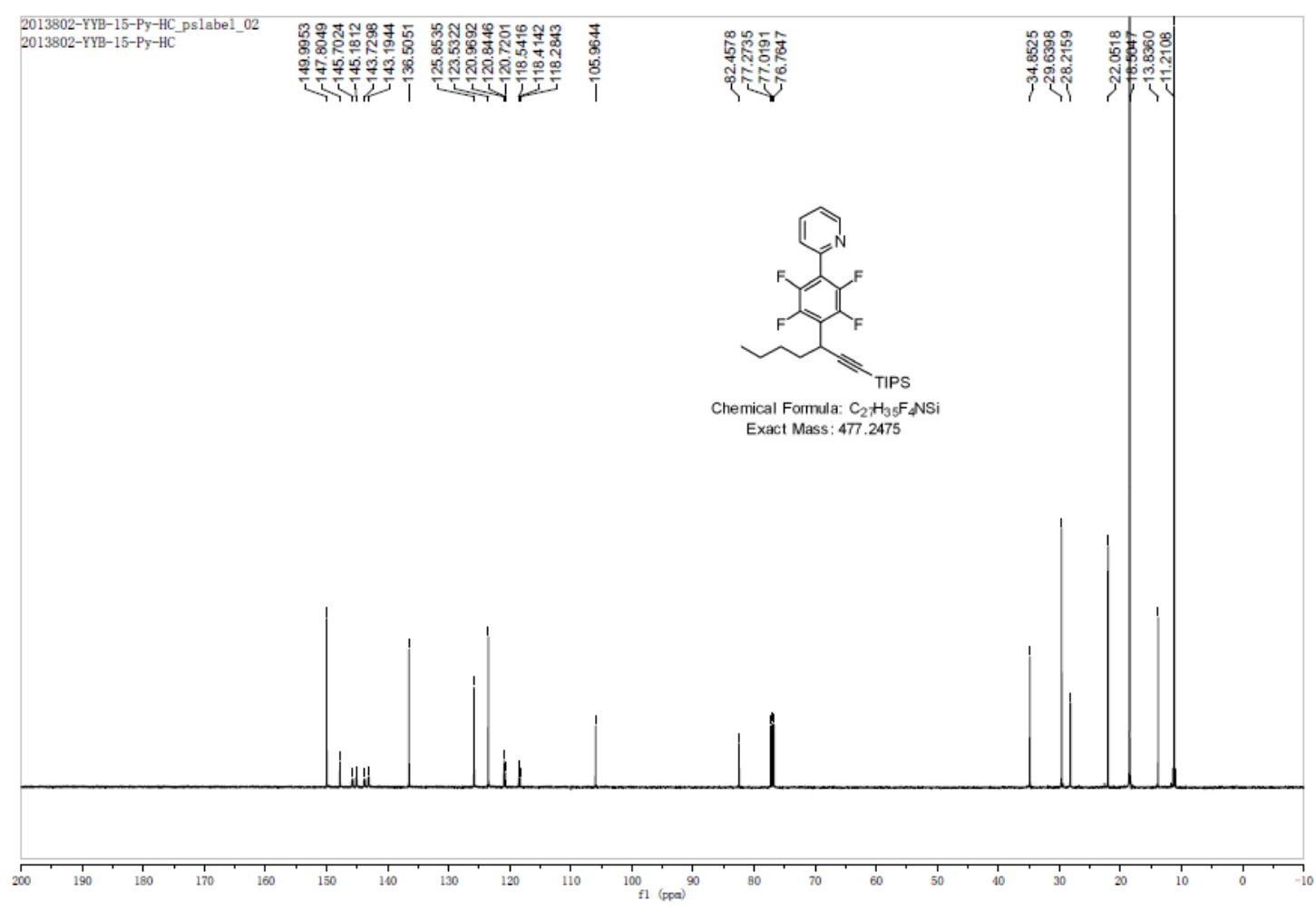

Triisopropyl(3-(2,3,5,6-tetrafluoro-4-(5-methylthiophen-2-yl)phenyl)tetradeca-1,11-diyn-1yl)silane (4k)

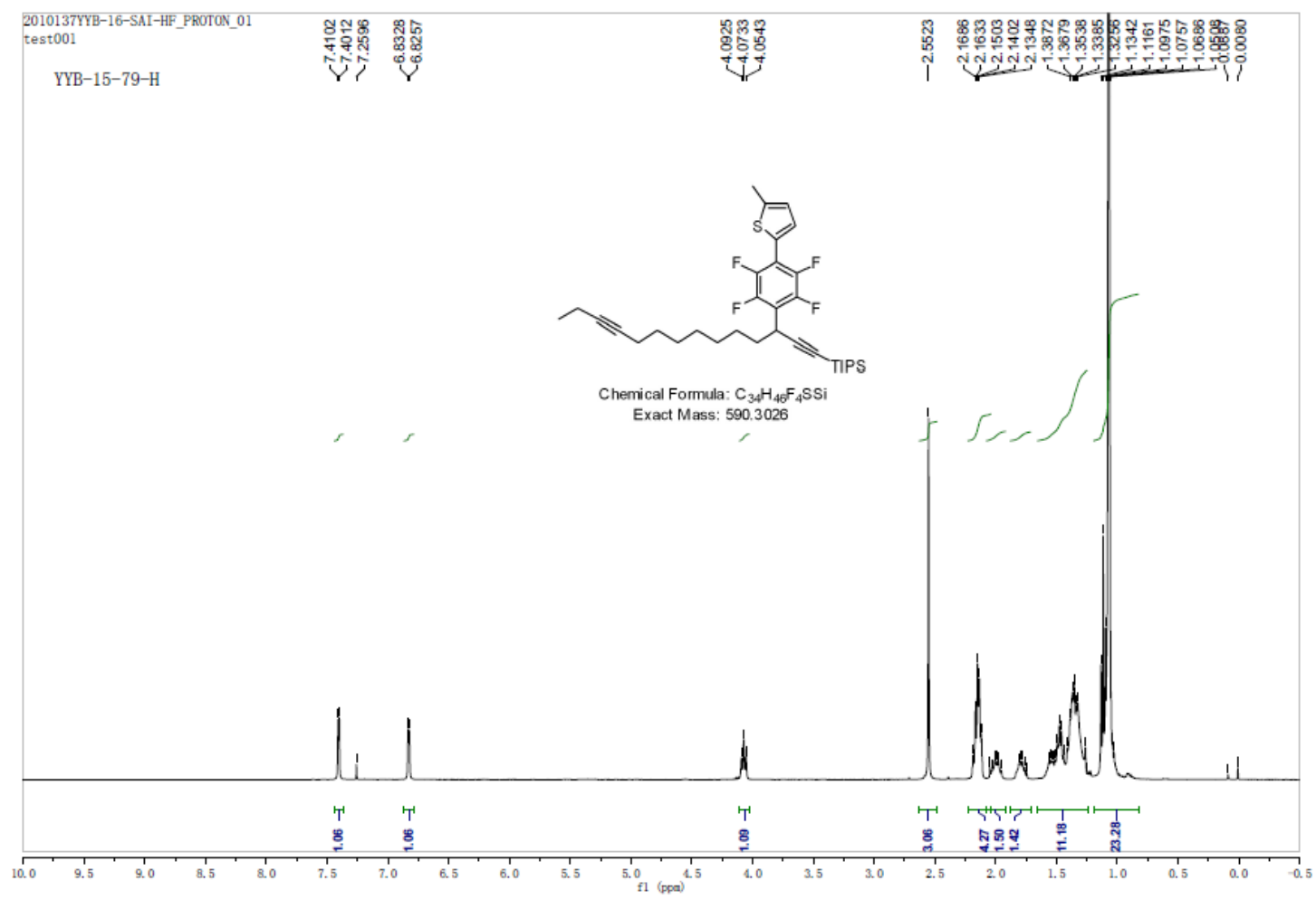


2010137YYB-16-SAI-HF_FLUORINE_01
test001

YYB-15-79-F

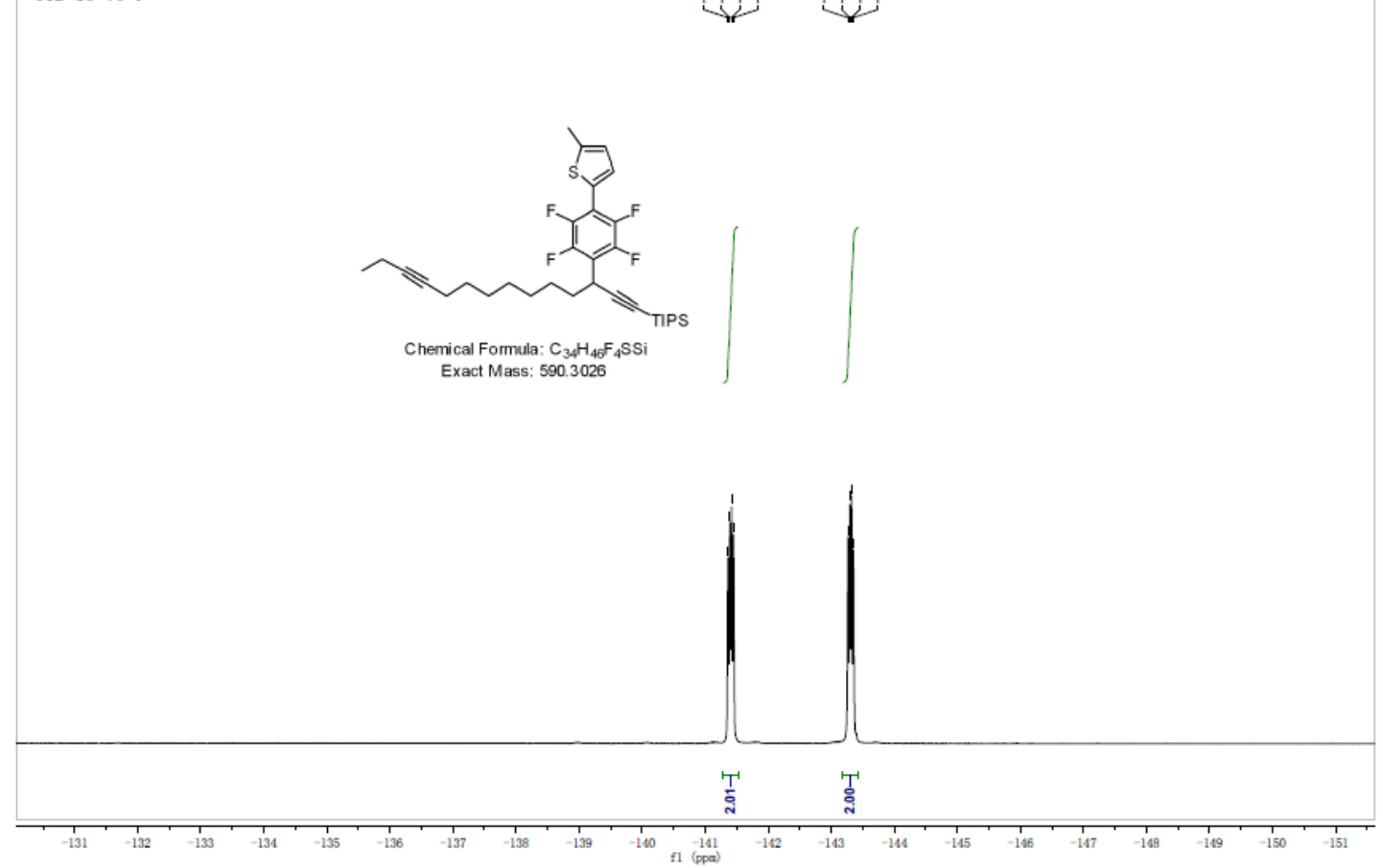


(Z)-(3-(4-(5-Butylfuran-2-yl)-2,3,5,6-tetrafluorophenyl)non-6-en-1-yn-1-yl)triisopropylsilane (4l)

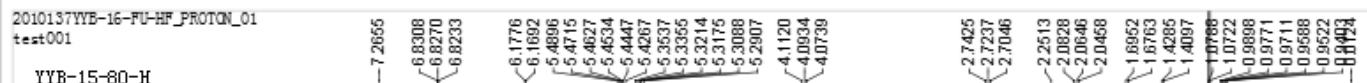

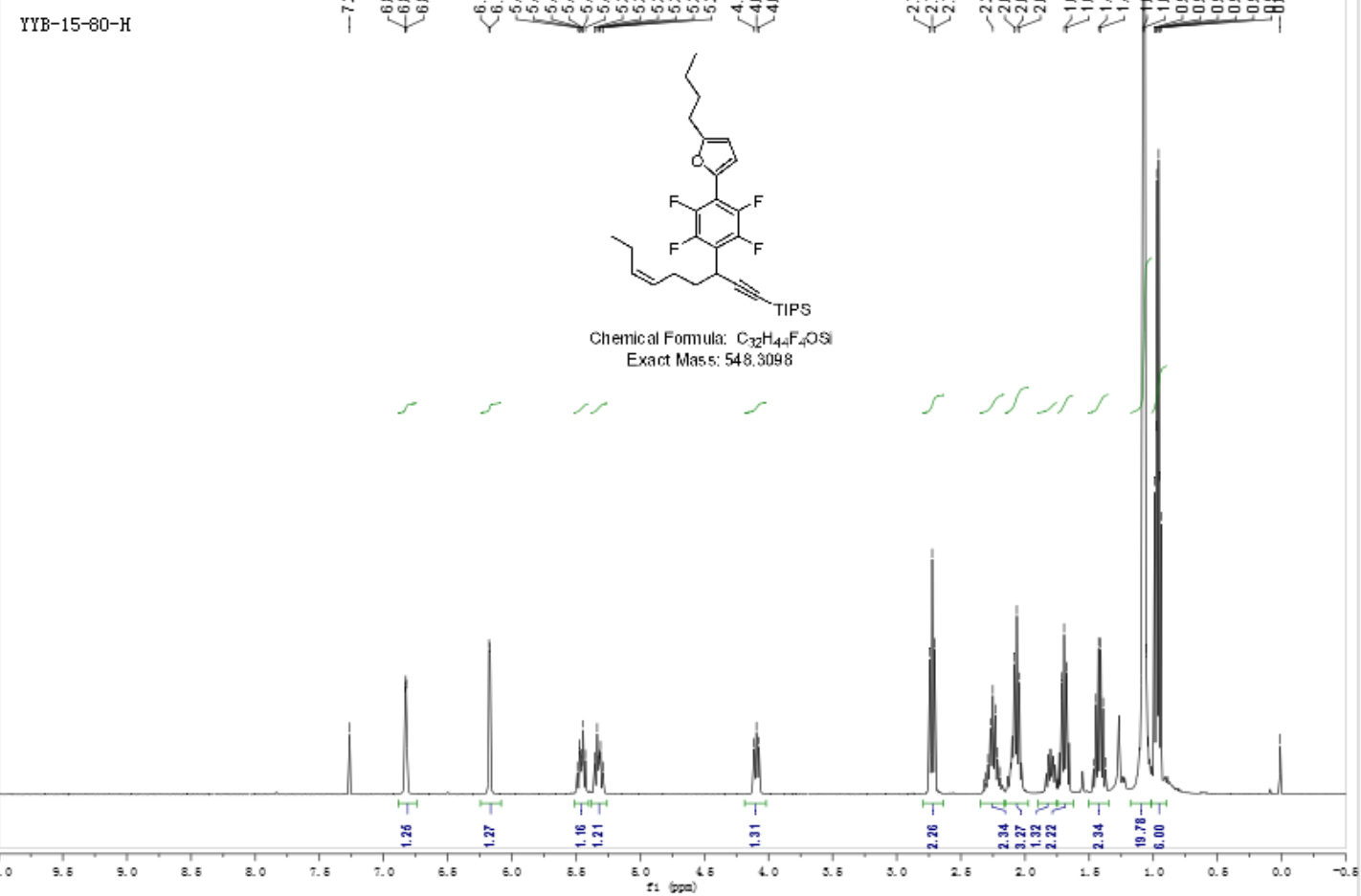

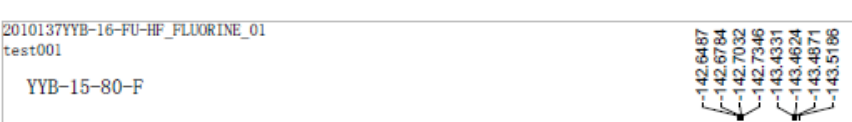

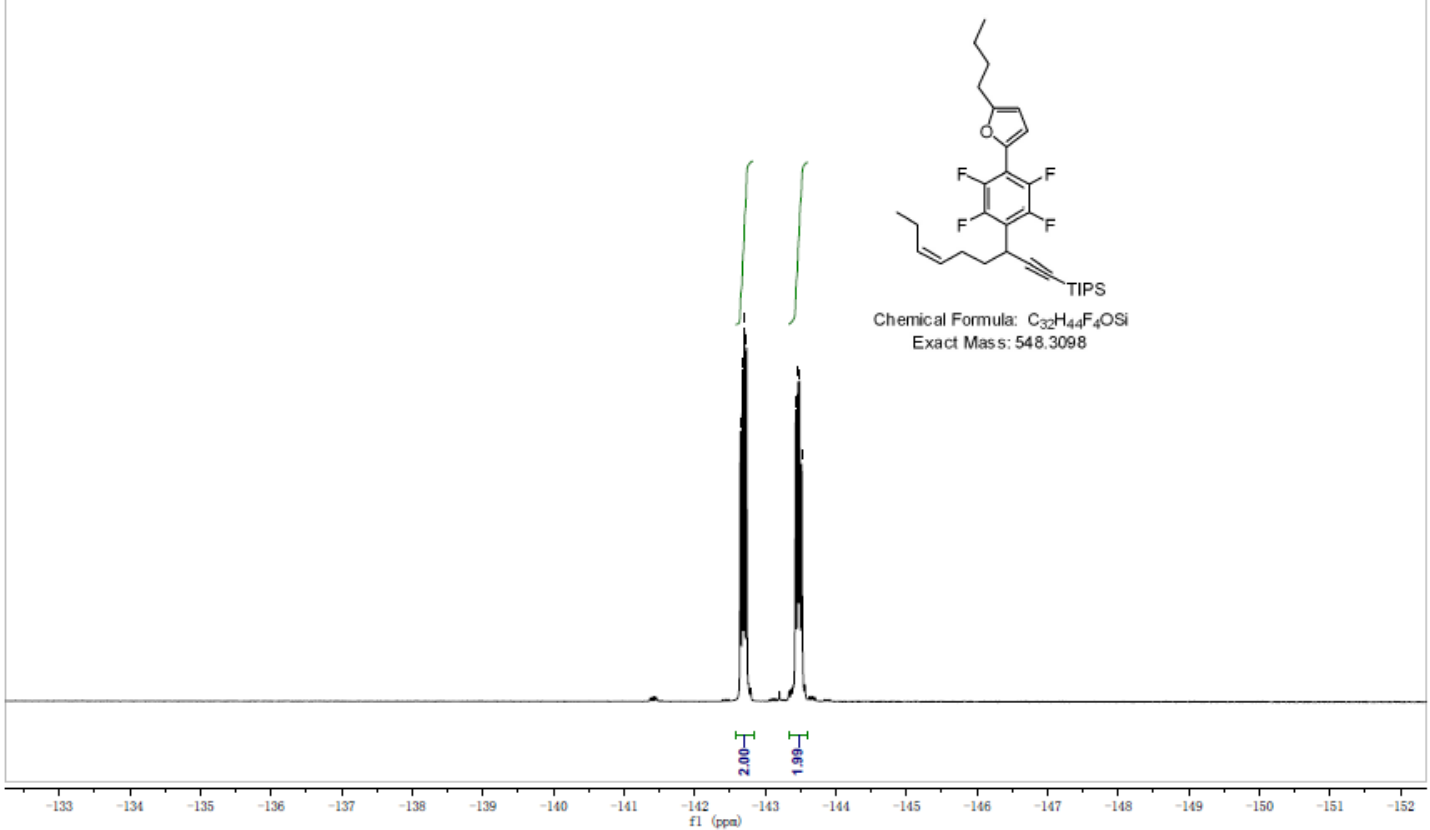




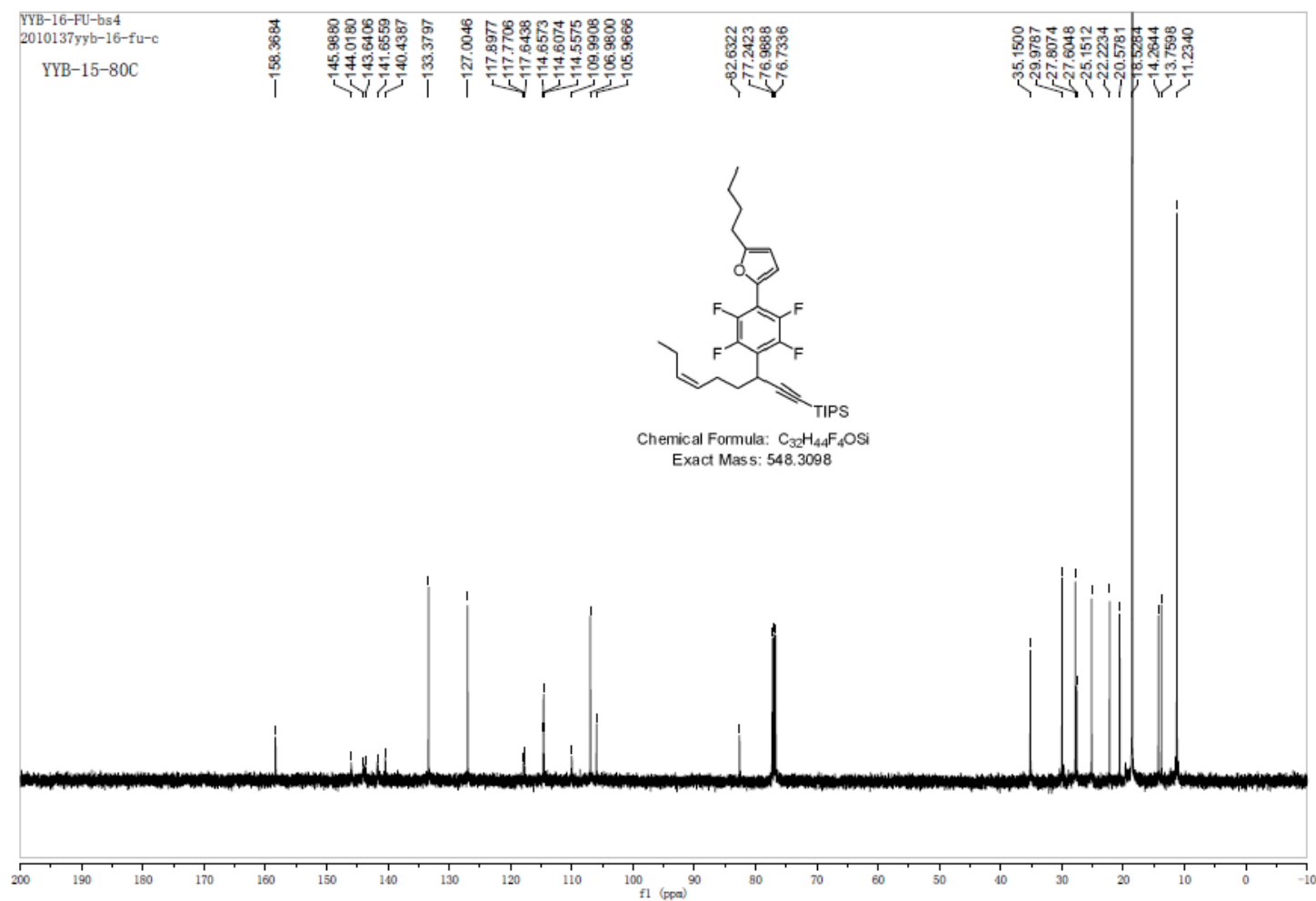

(3-(4-Cinnamyl-2,3,5,6-tetrafluorophenyl)hept-1-yn-1-yl)triisopropylsilane (6)

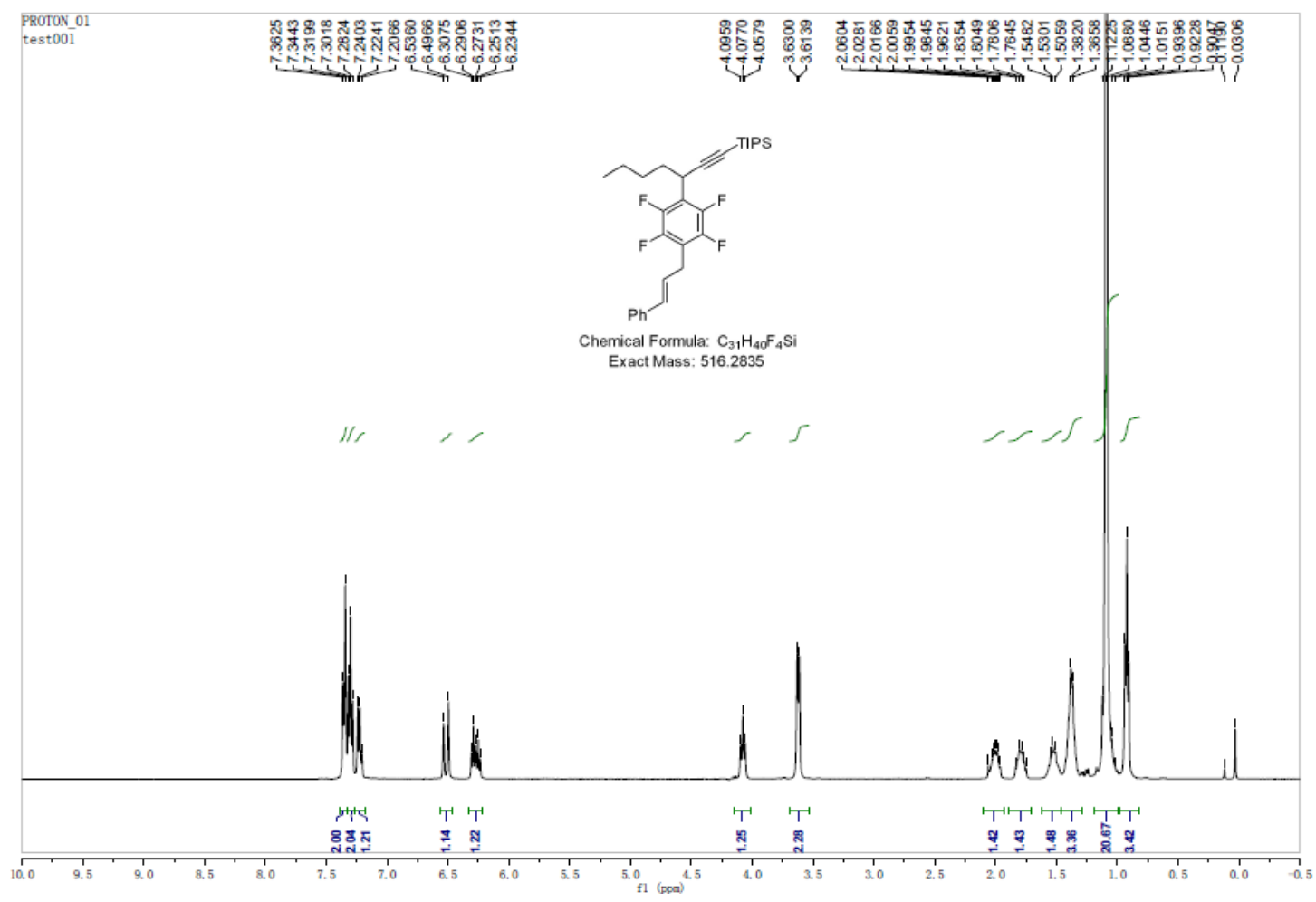



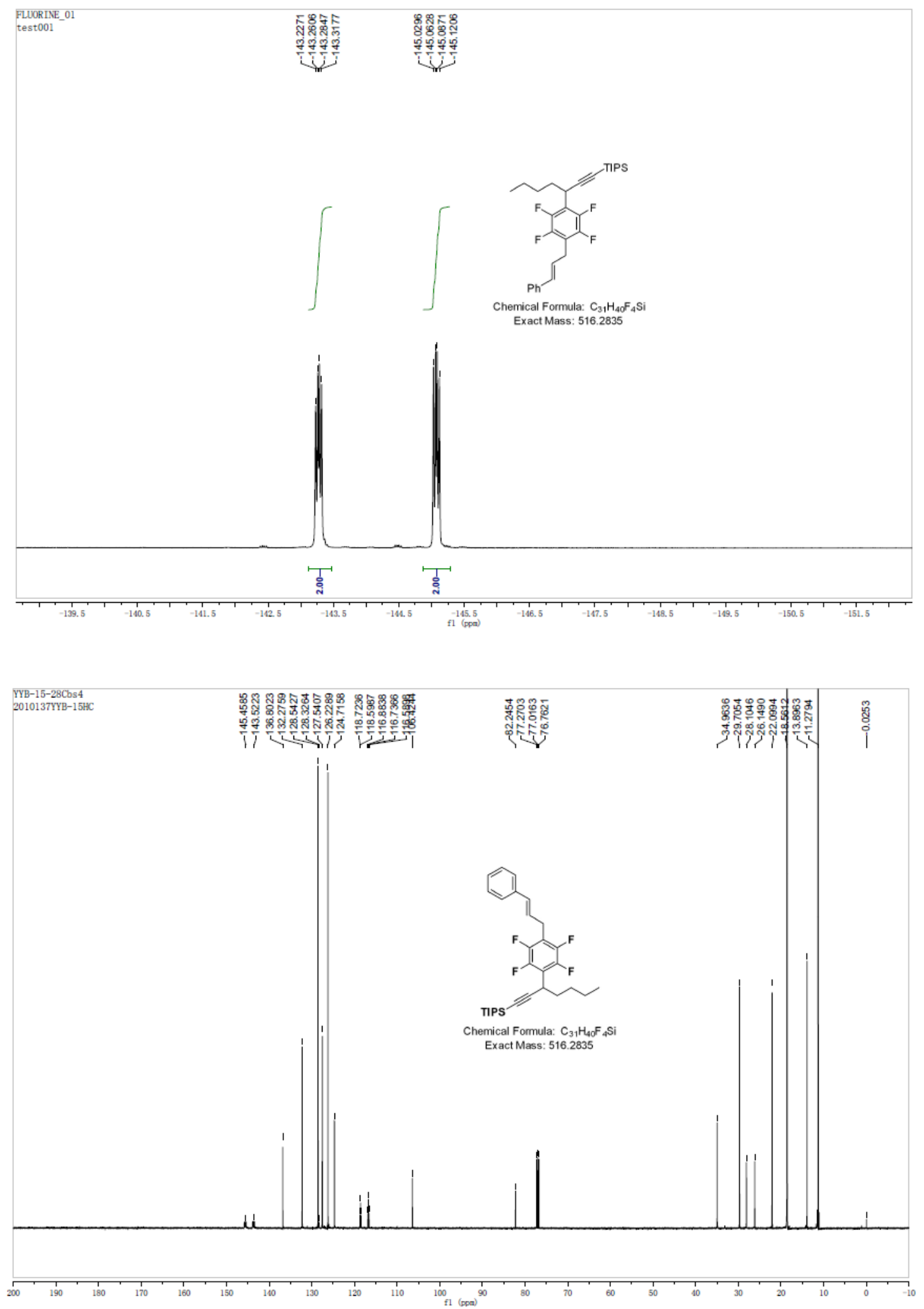
Triisopropyl(3-(2,3,5,6-tetrafluoro-4-(4-methoxybenzyl)phenyl)hept-1-yn-1-yl)silane (8)
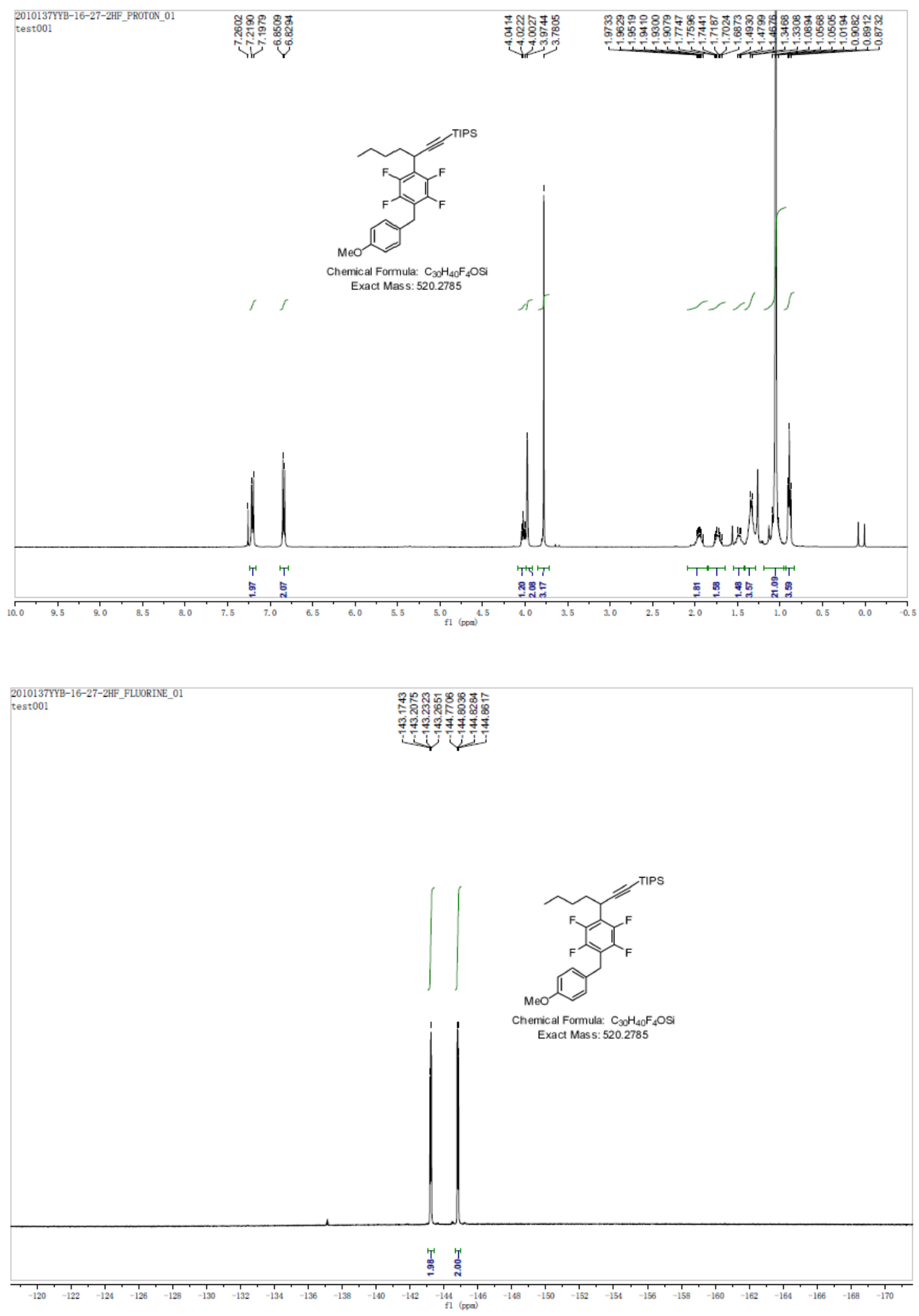


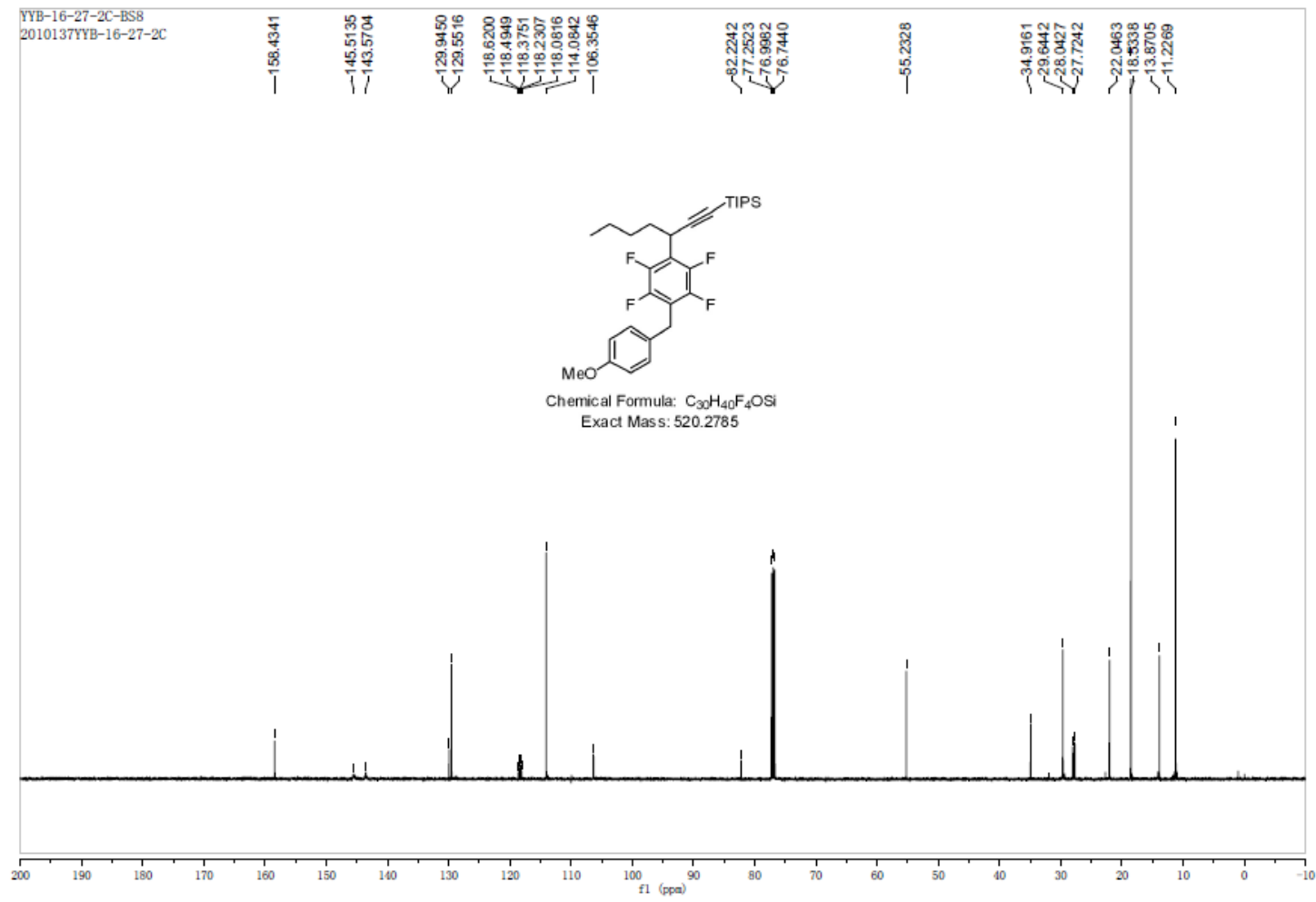

1,2,3,4,5-Pentafluoro-6-(5-phenylpent-1-yn-3-yl)benzene (9)

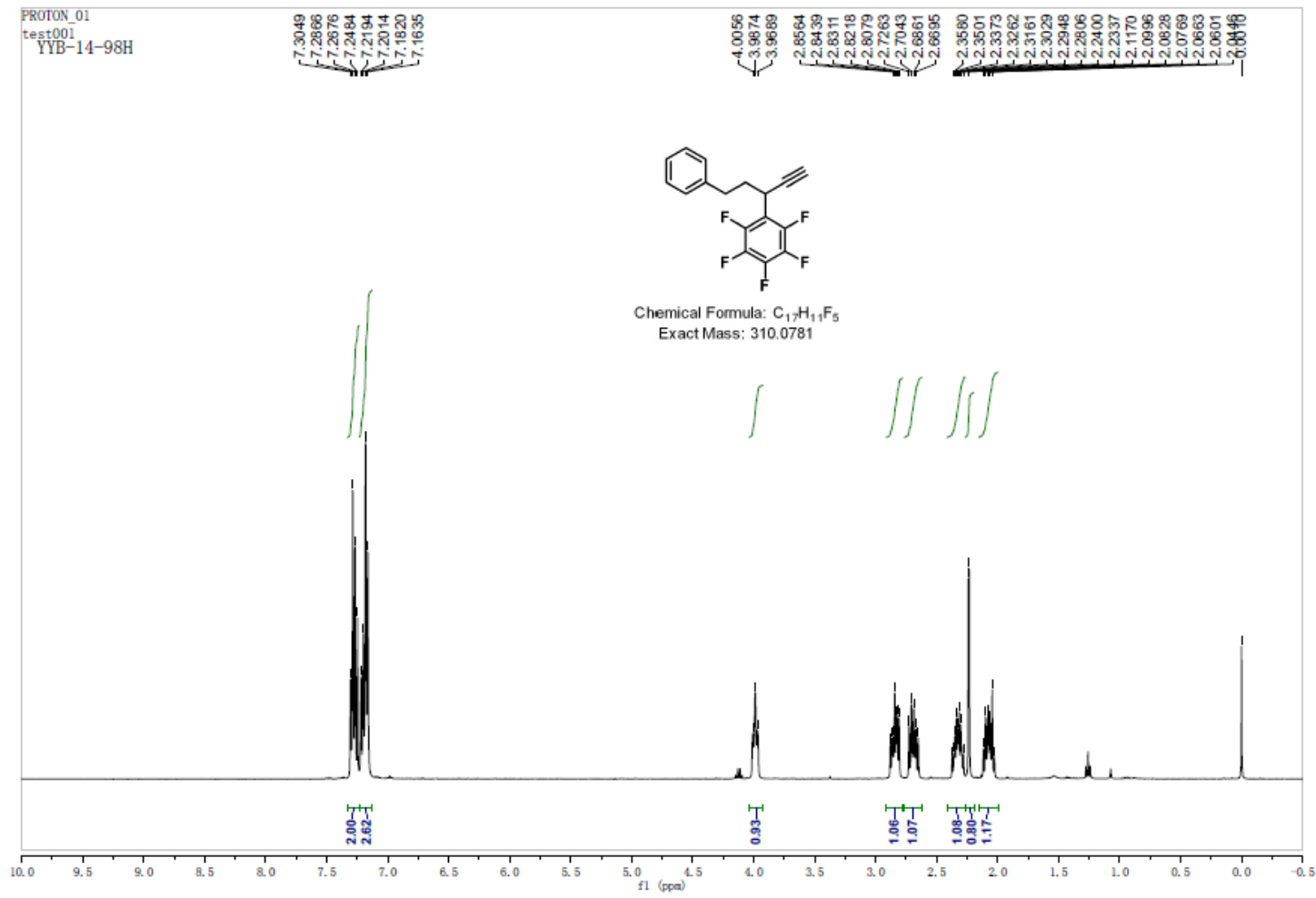




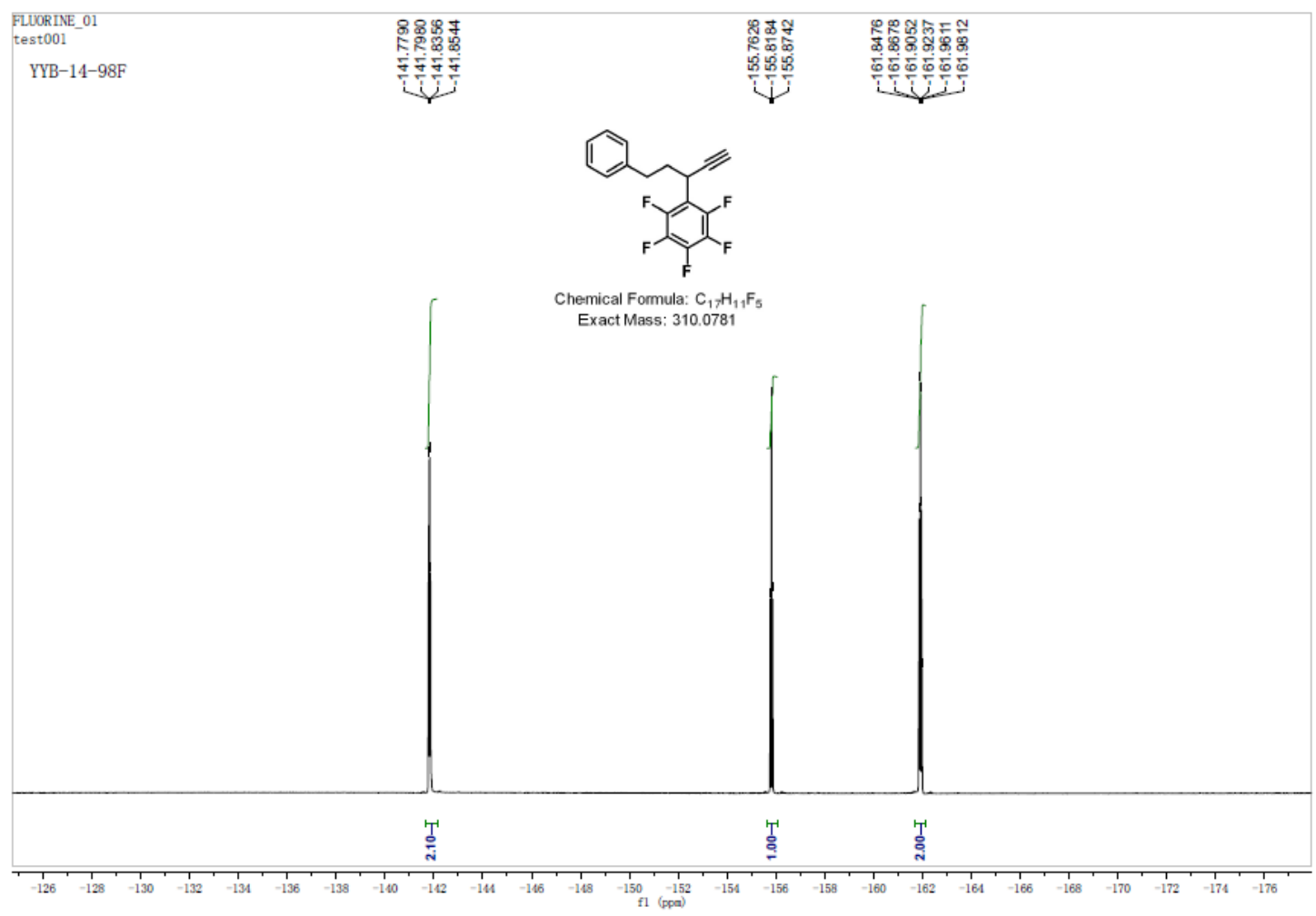

\begin{tabular}{|c|c|c|c|}
\hline $\begin{array}{l}\text { YYB-14-98C-bs7 } \\
\text { Gradient Shimming }\end{array}$ & 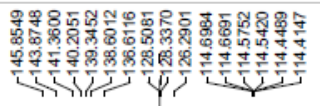 & 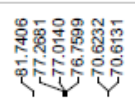 & 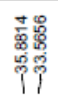 \\
\hline
\end{tabular}
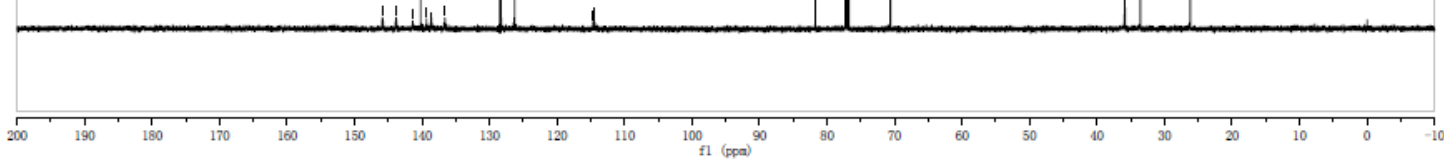
1,2,3,4,5-Pentafluoro-6-(5-phenylpent-1-en-3-yl)benzene (10a)
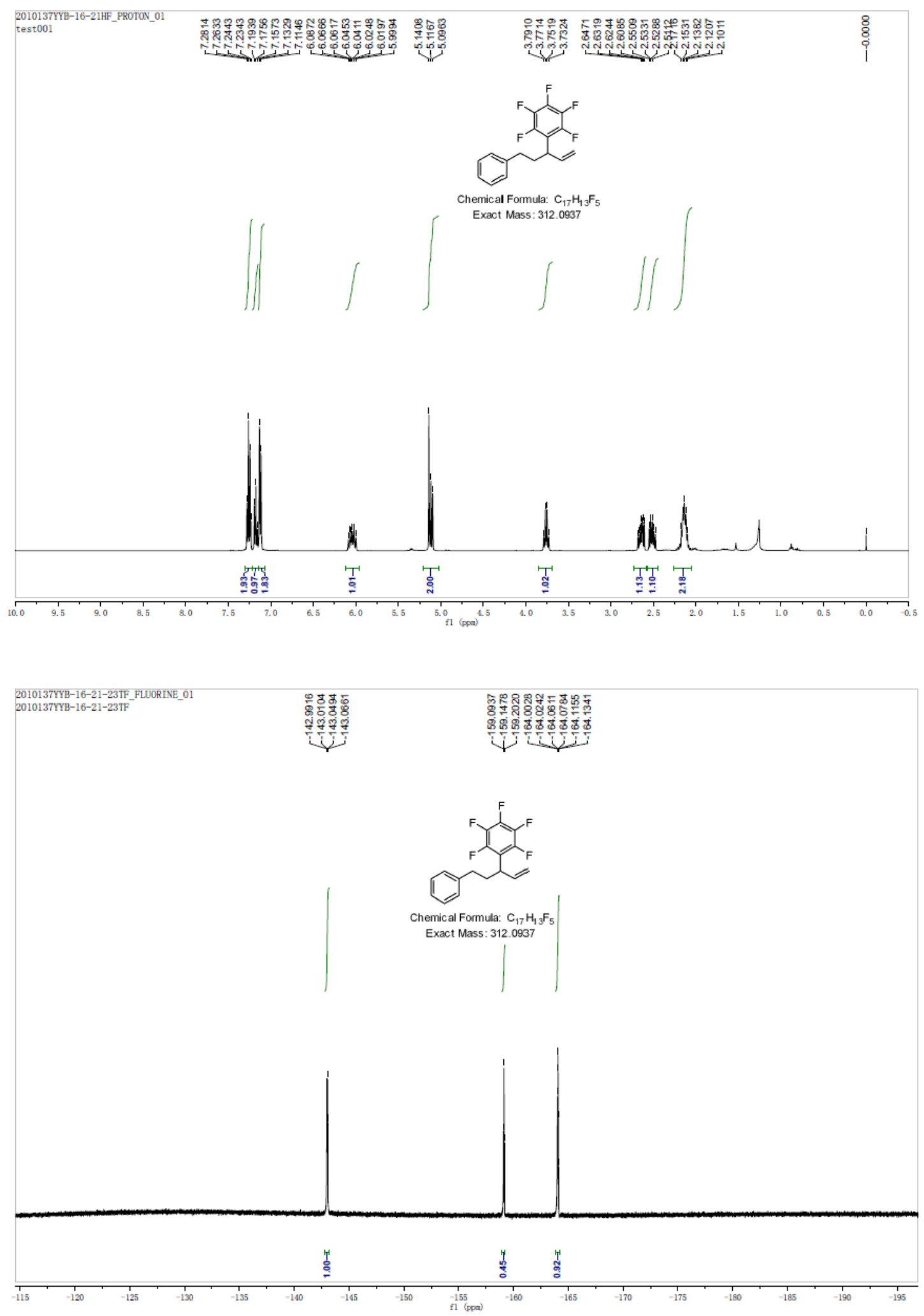


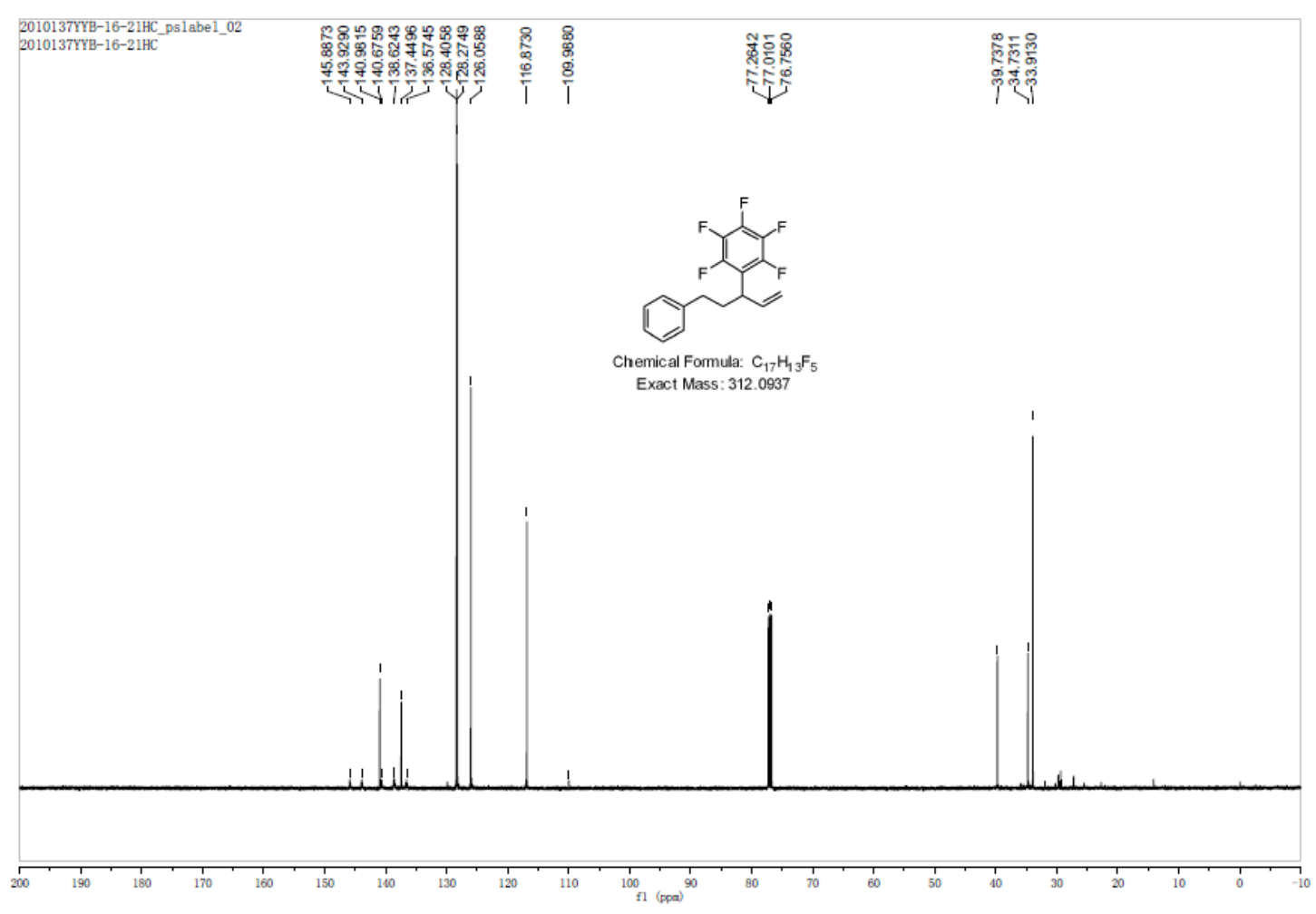

1,2,3,4,5-Pentafluoro-6-(1-phenylpentan-3-yl)benzene (10b)

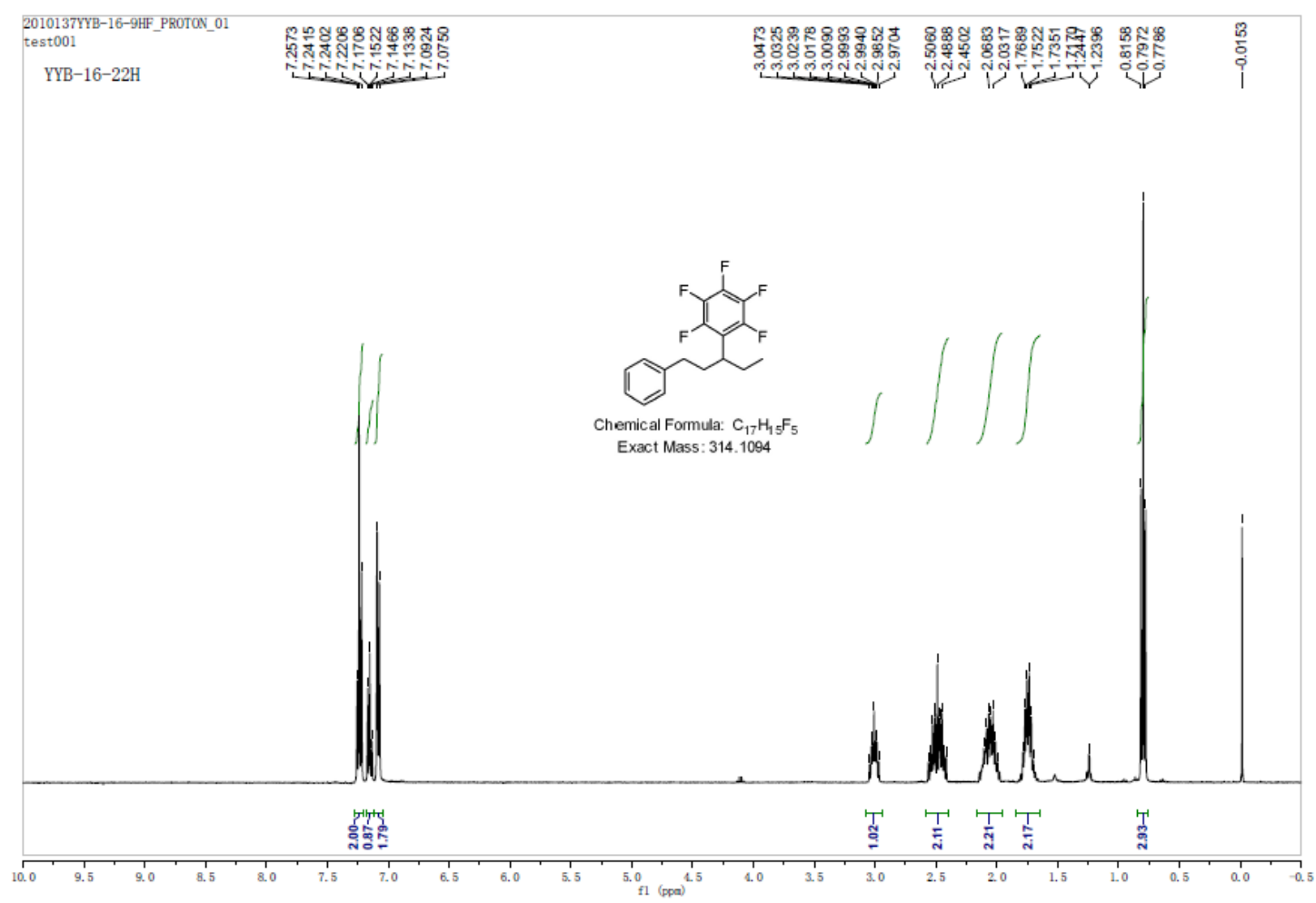


2010137YYB-16-9HF_FLORINE_01

test001

YYB-16-22F

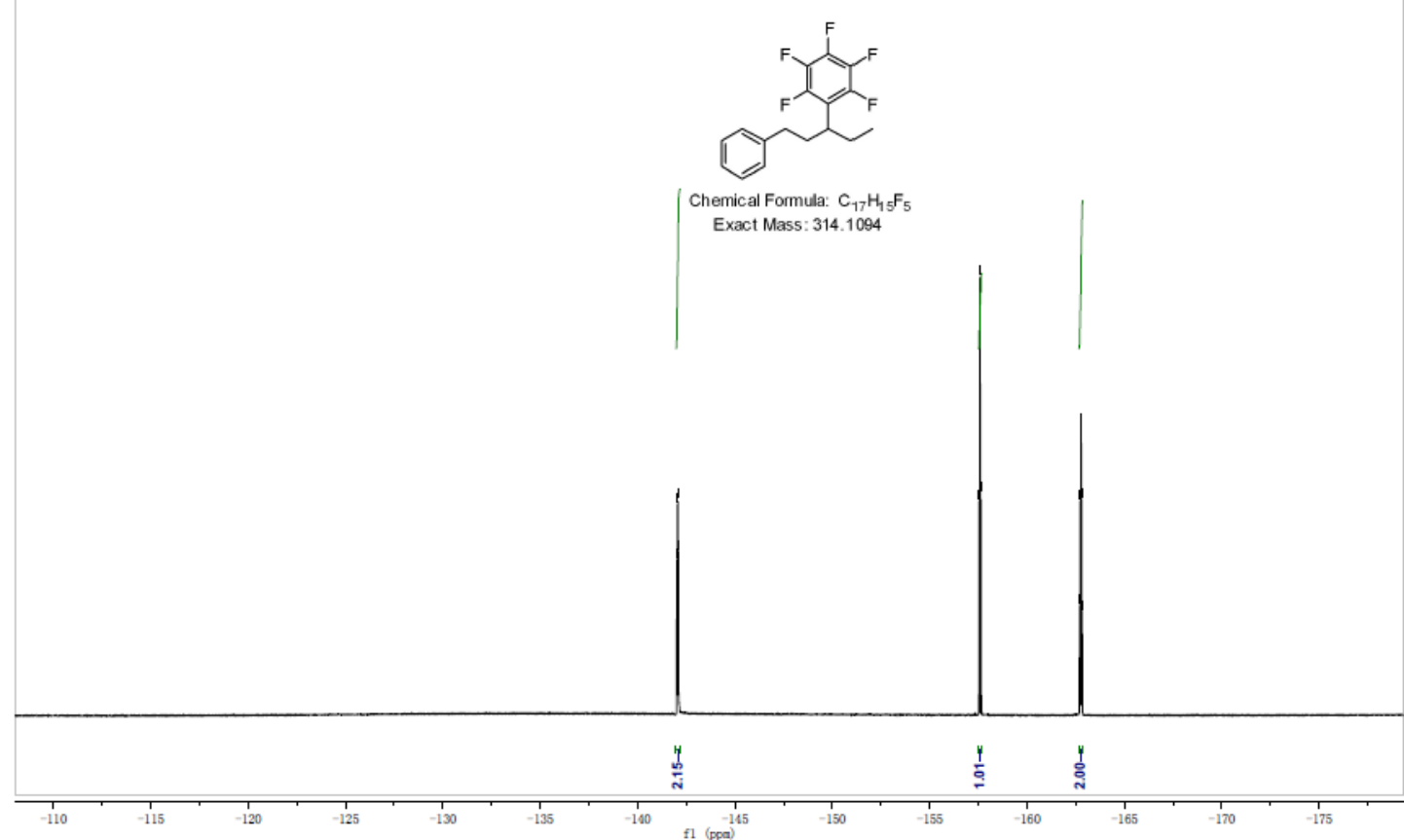

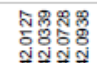

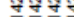

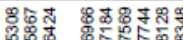

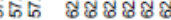

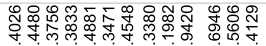

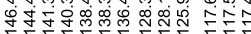

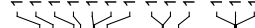

2010137YYB-16-9-BS2-C

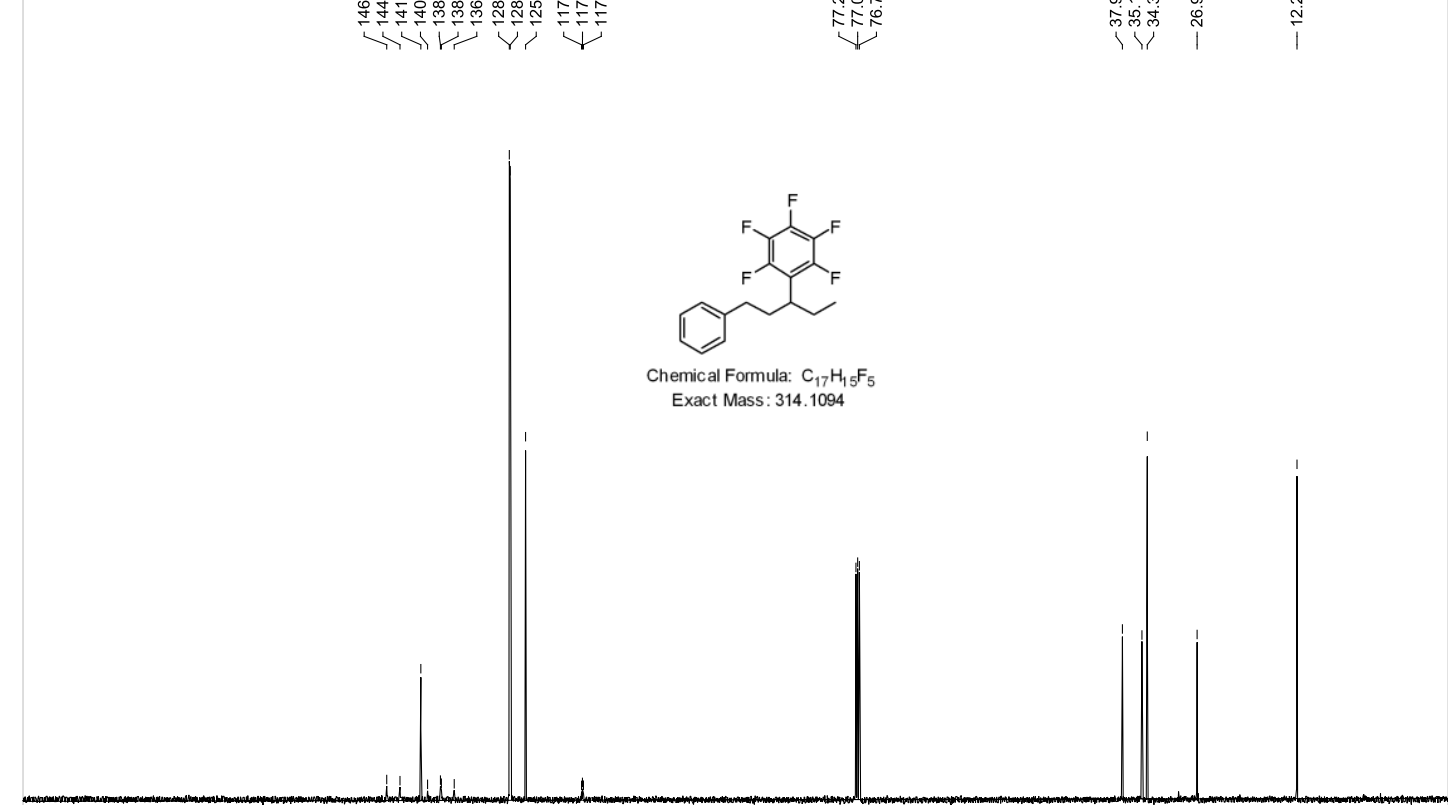

${ }_{\mathrm{f1}}^{100}(\mathrm{ppm})$ 
3-(Perfluorophenyl)-5-phenylpentan-2-one (10c)
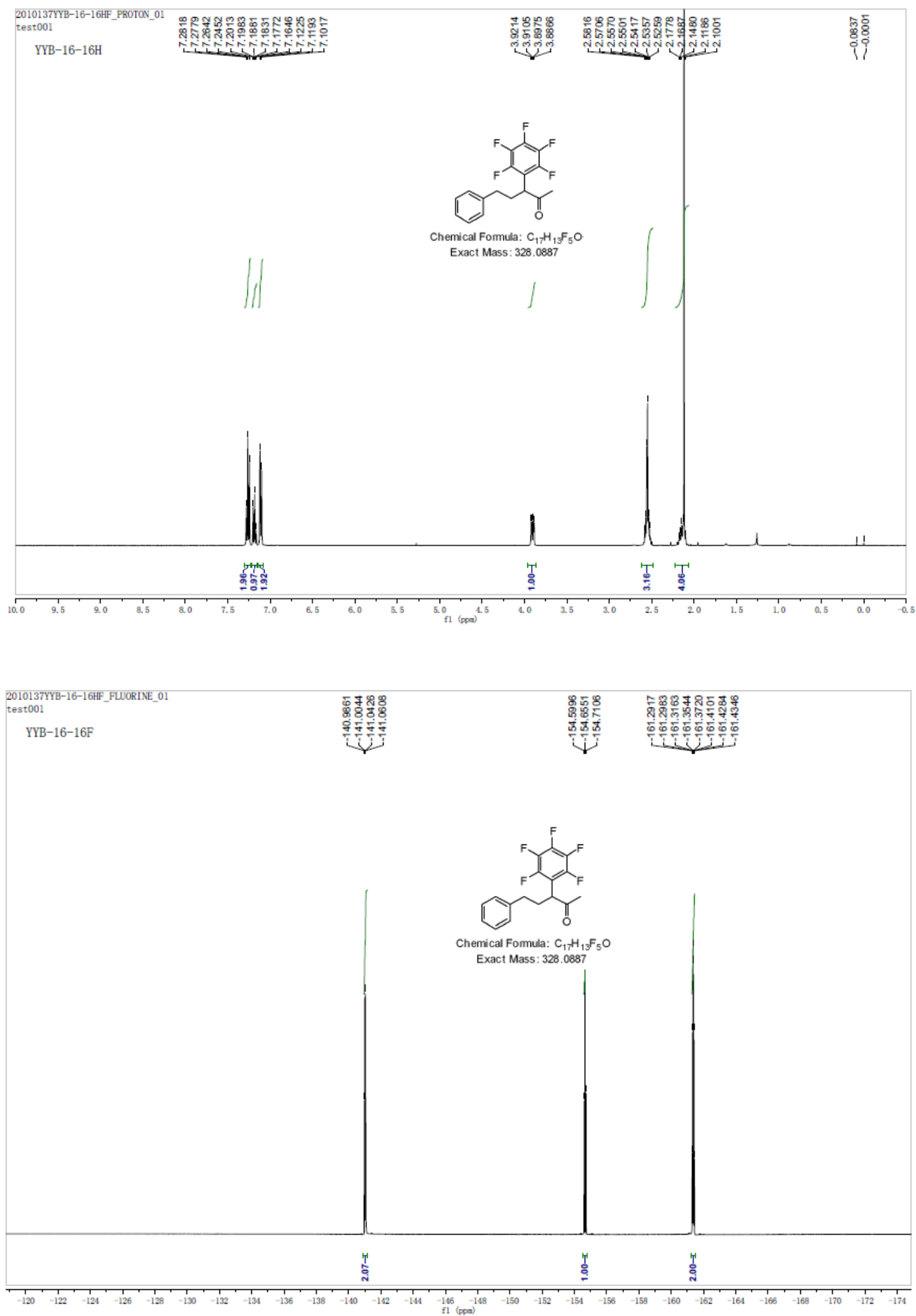


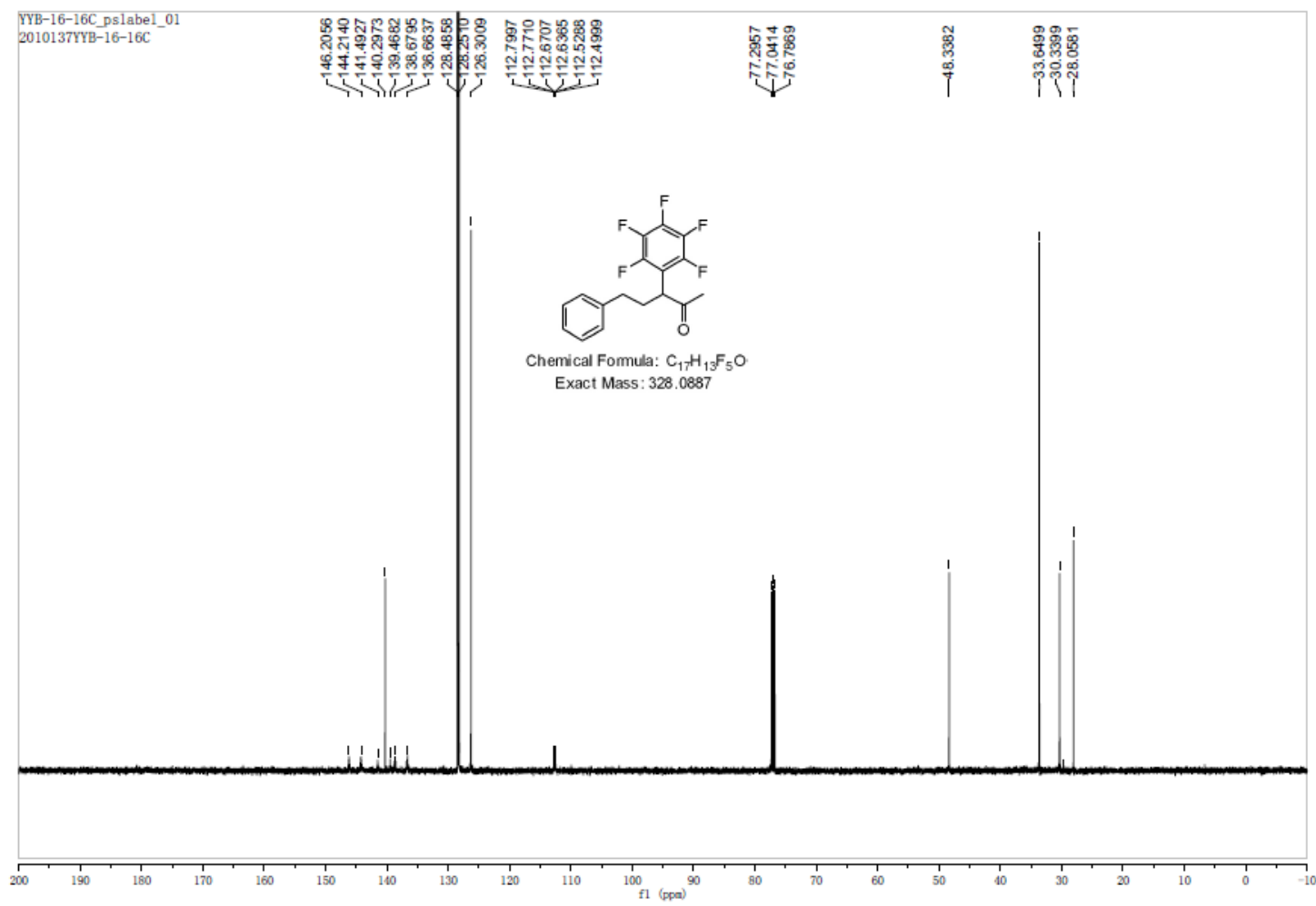

Methyl 3-(perfluorophenyl)-5-phenylpentanoate (10d)

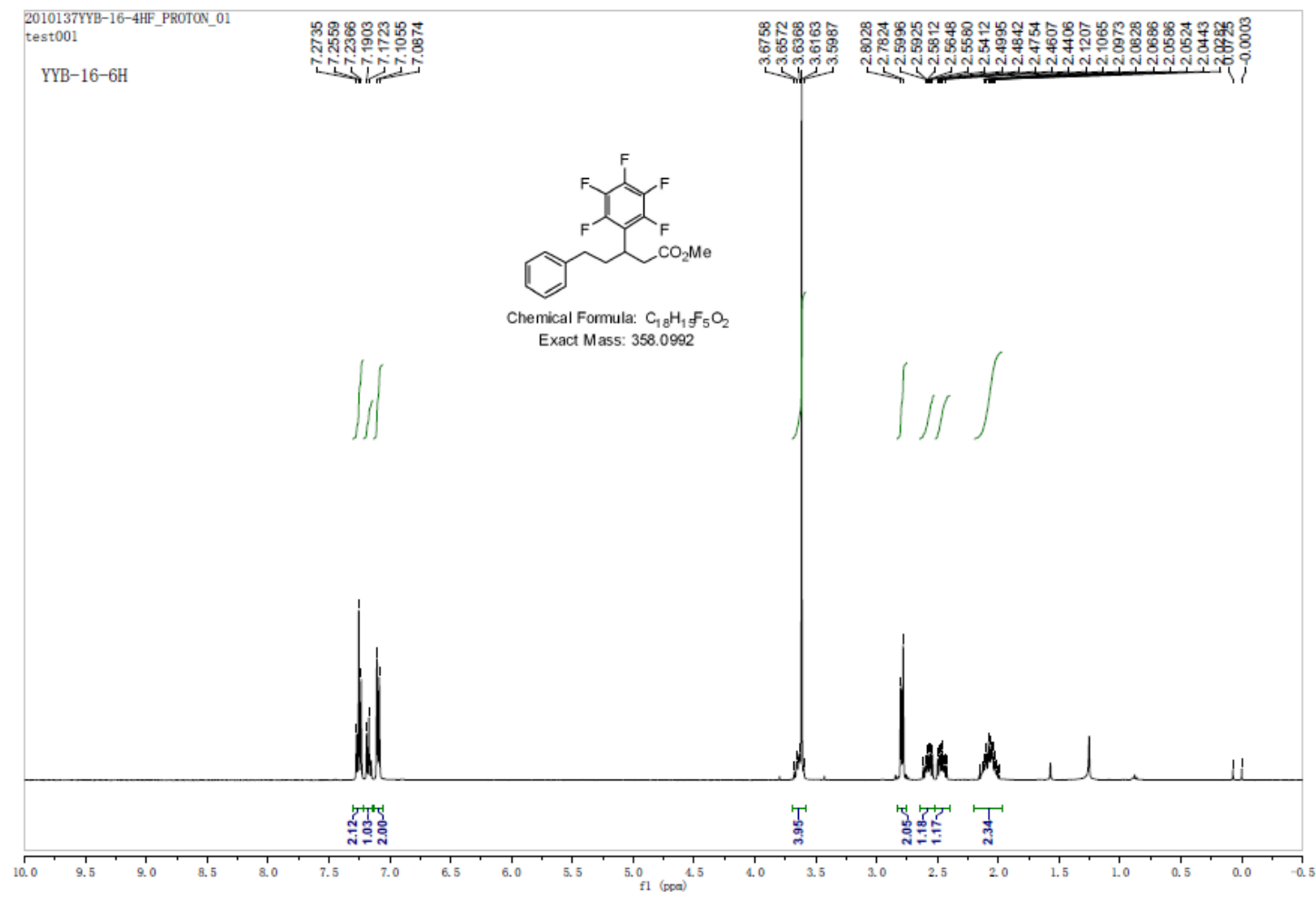



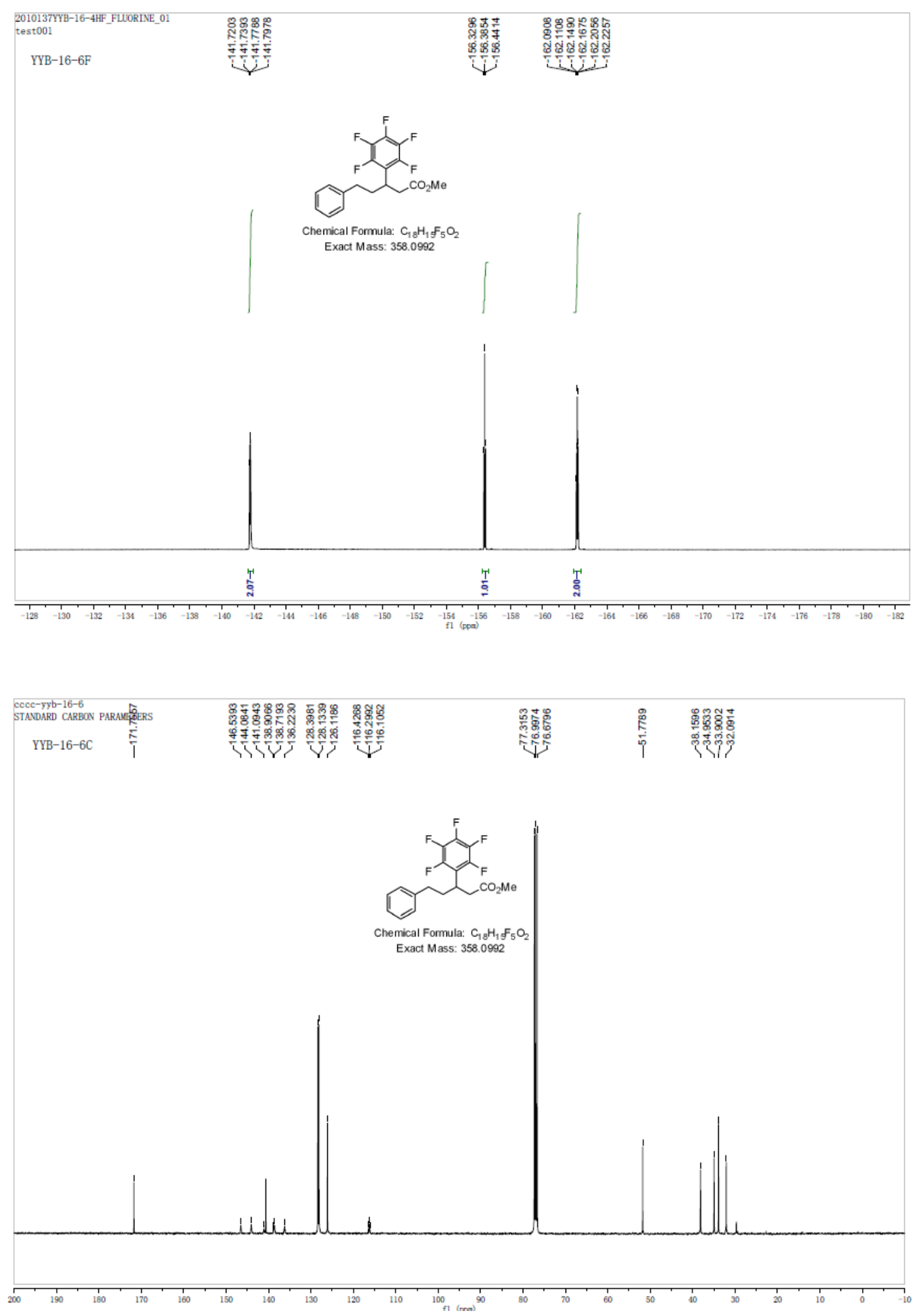
2-Methyl-5-(1-(perfluorophenyl)-3-phenylpropyl)oxazole (10e)
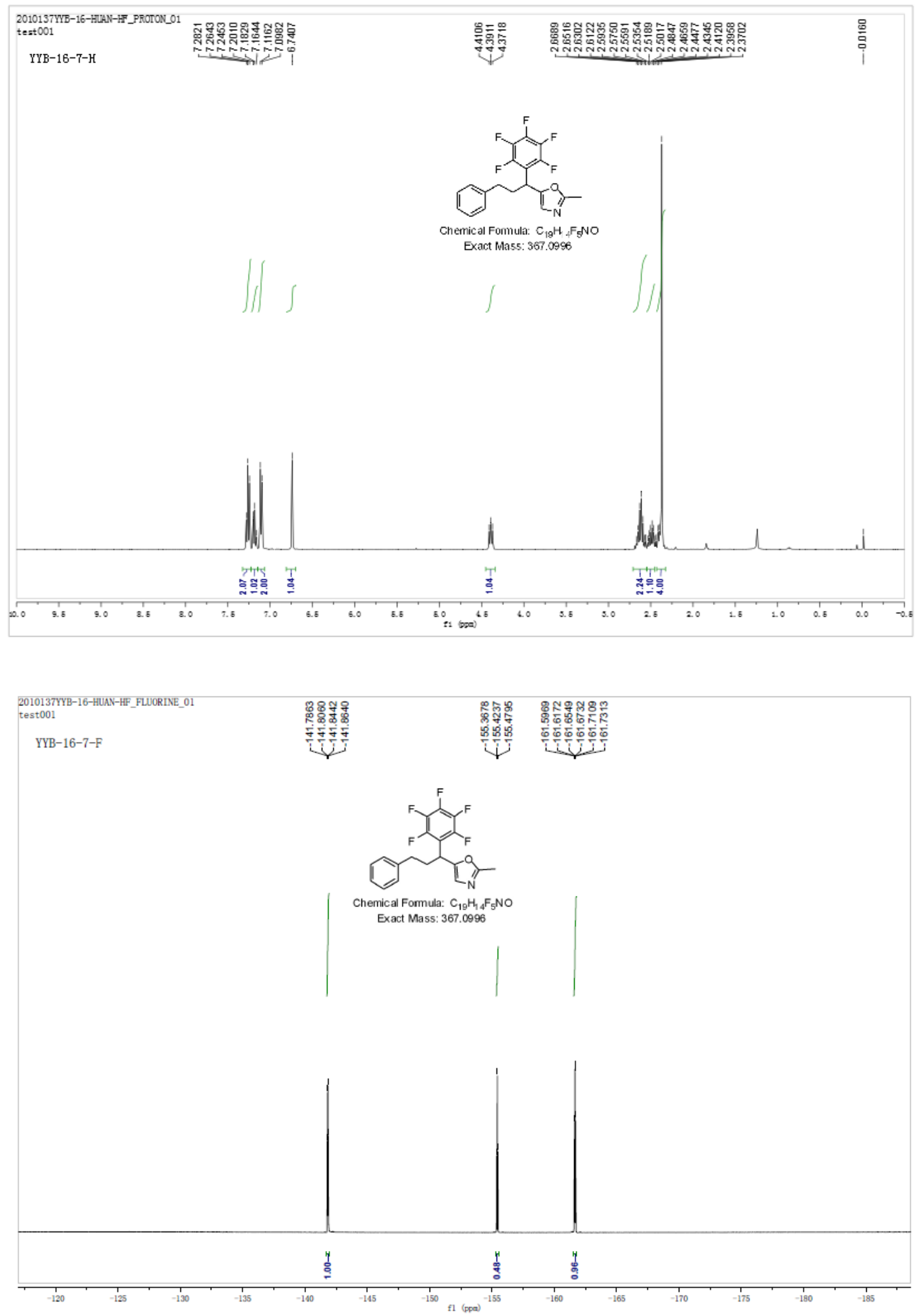


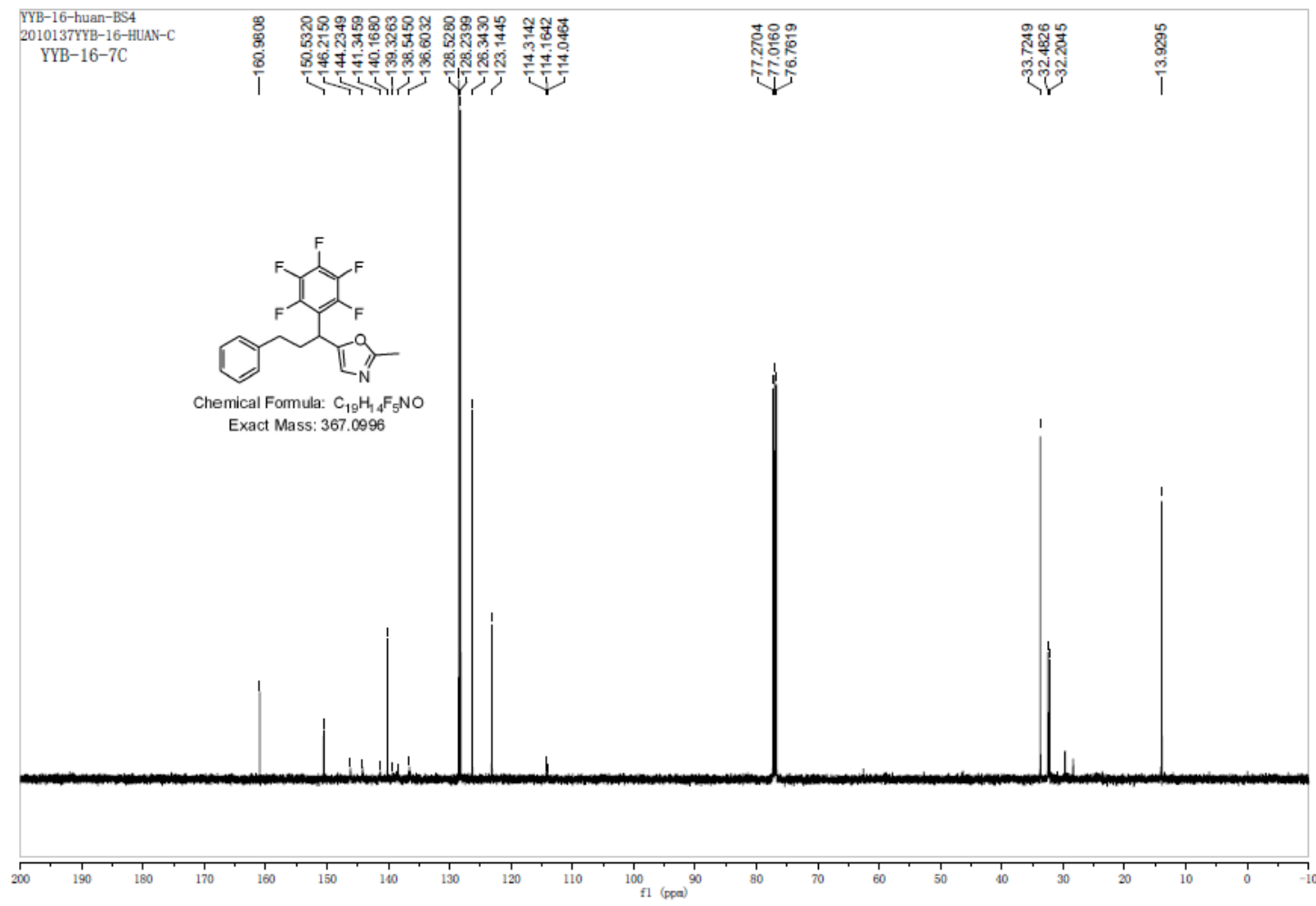

5-(Dimethylamino)-n-(3-(4-(1-(perfluorophenyl)-3-phenylpropyl)-1h-1,2,3-triazol-1yl)propyl)naphthalene-1-sulfonamide (10f)

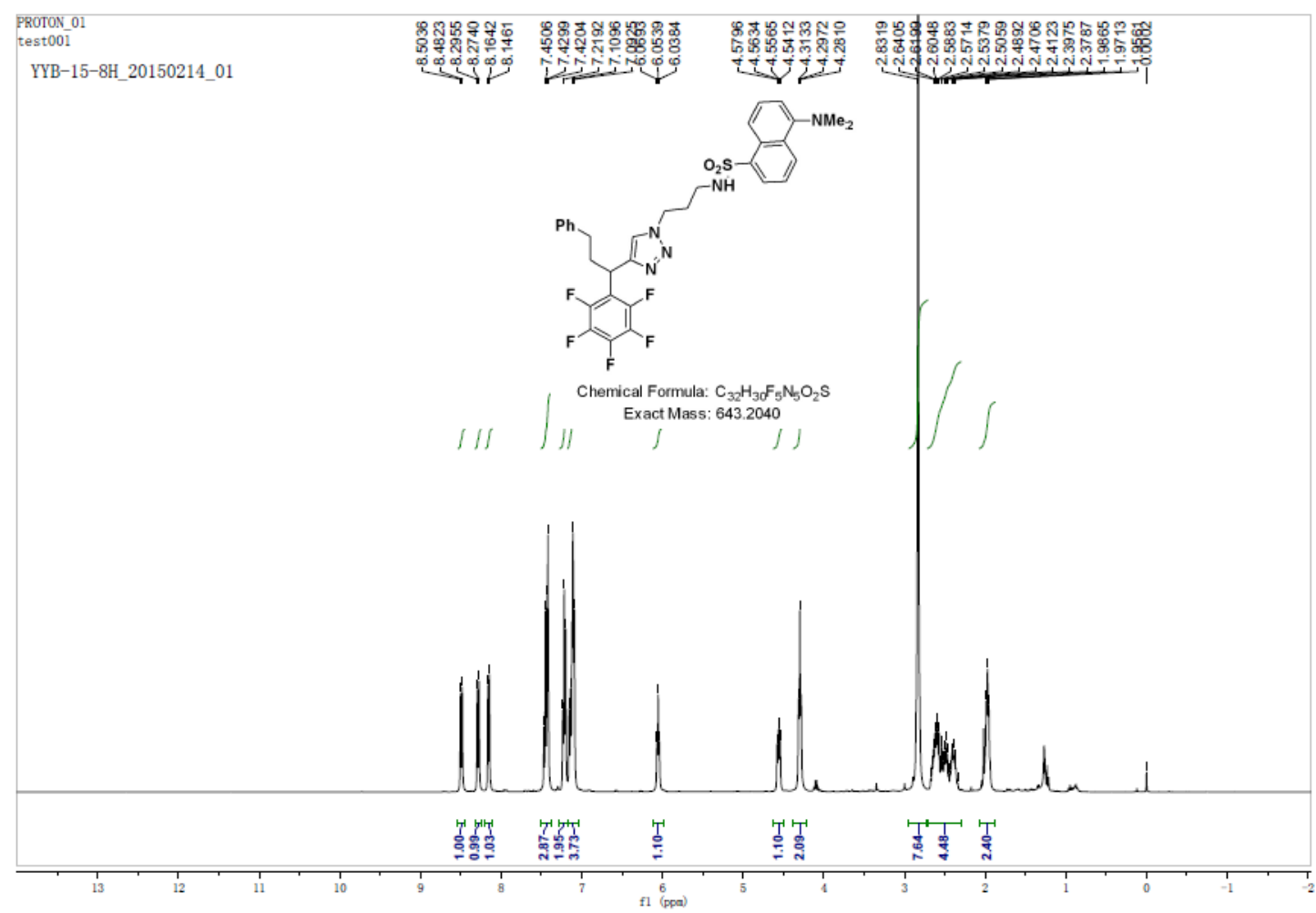




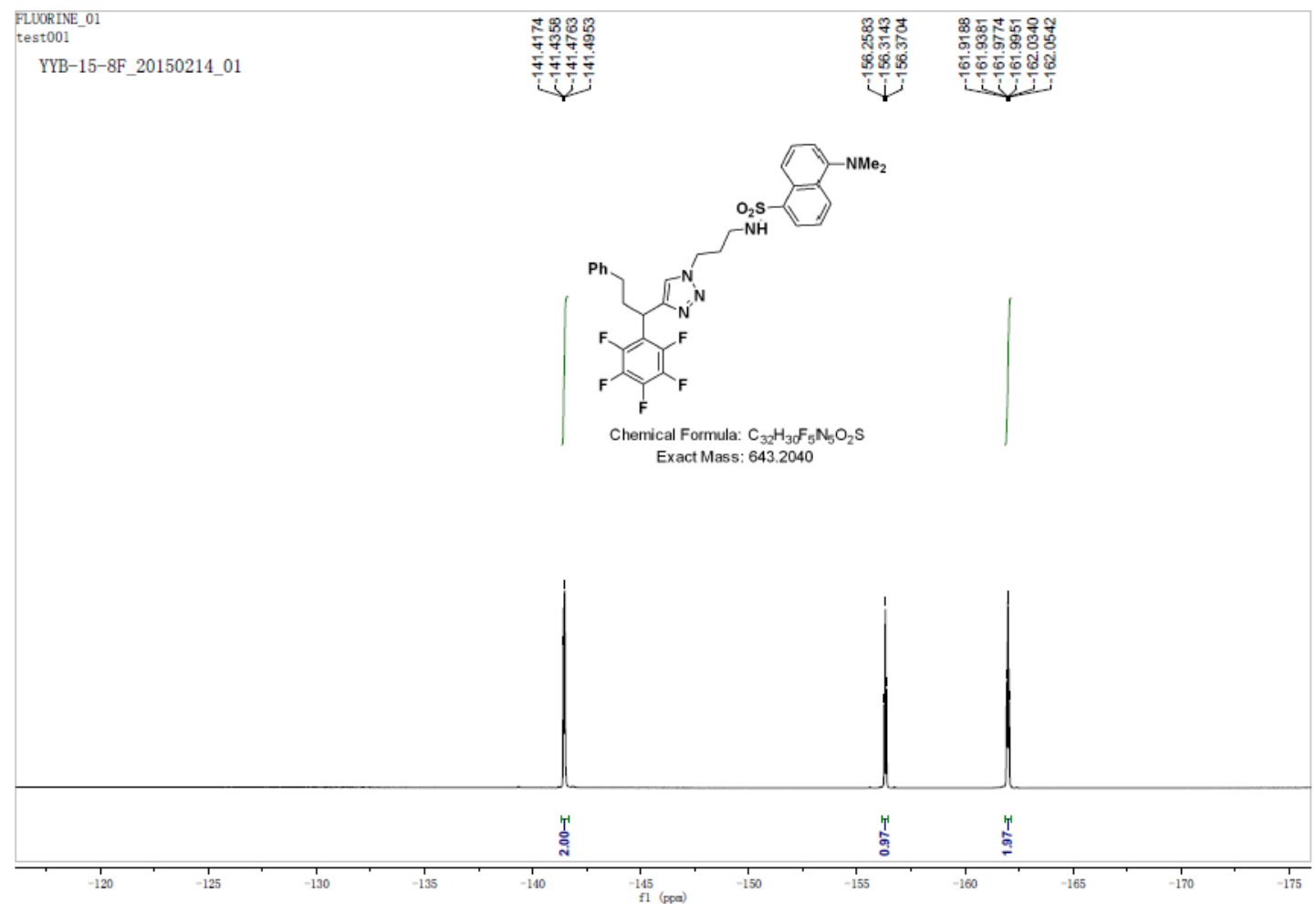

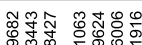

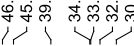

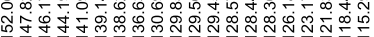
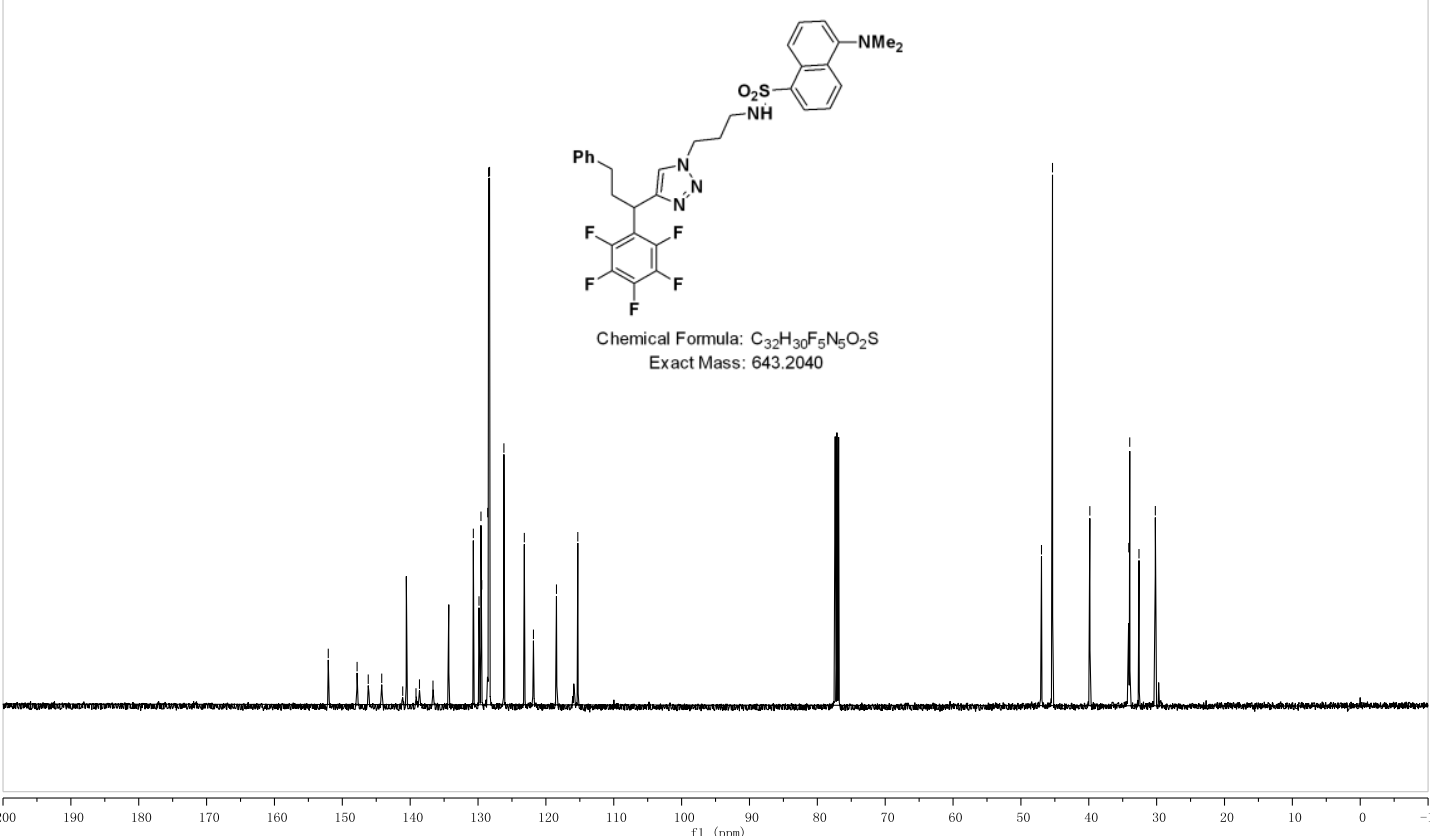
(R)-5-Phenyl-1-(triisopropylsilyl)pent-1-yn-3-ol ( $R$-2da)
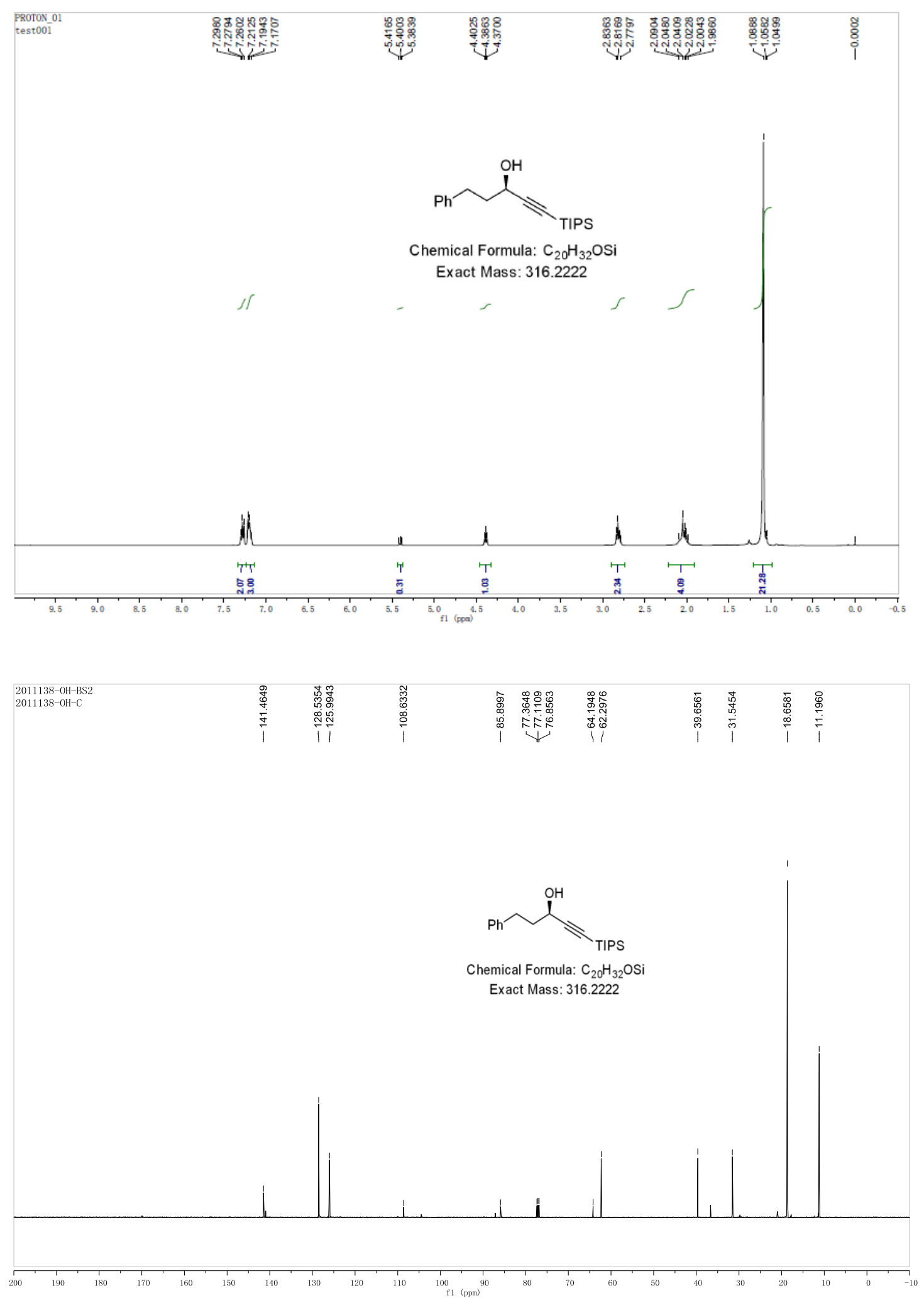

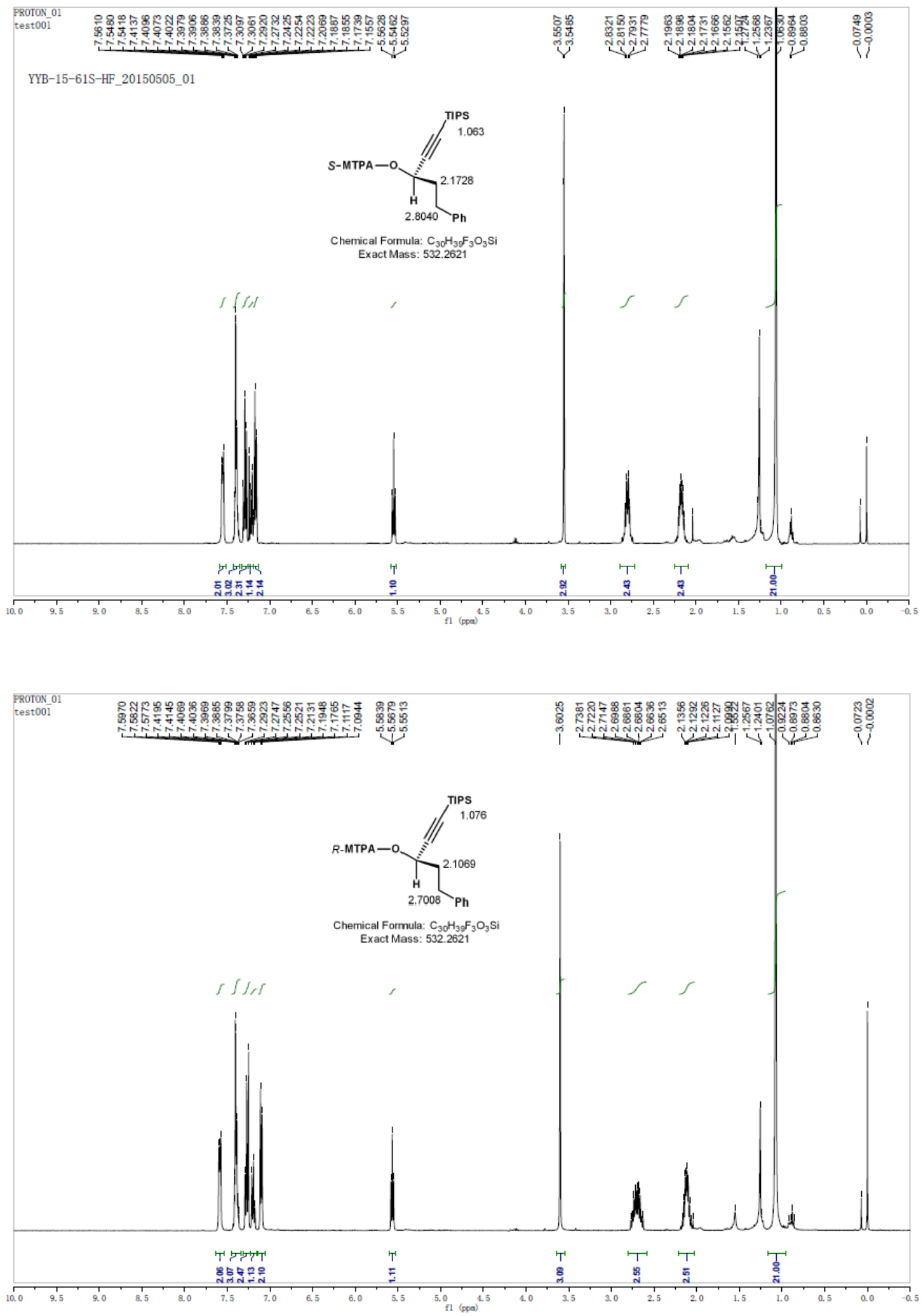
Copies of HPLC Analysis Spectra of Compounds $R$-2da, $R$-2d, and $S$-3d

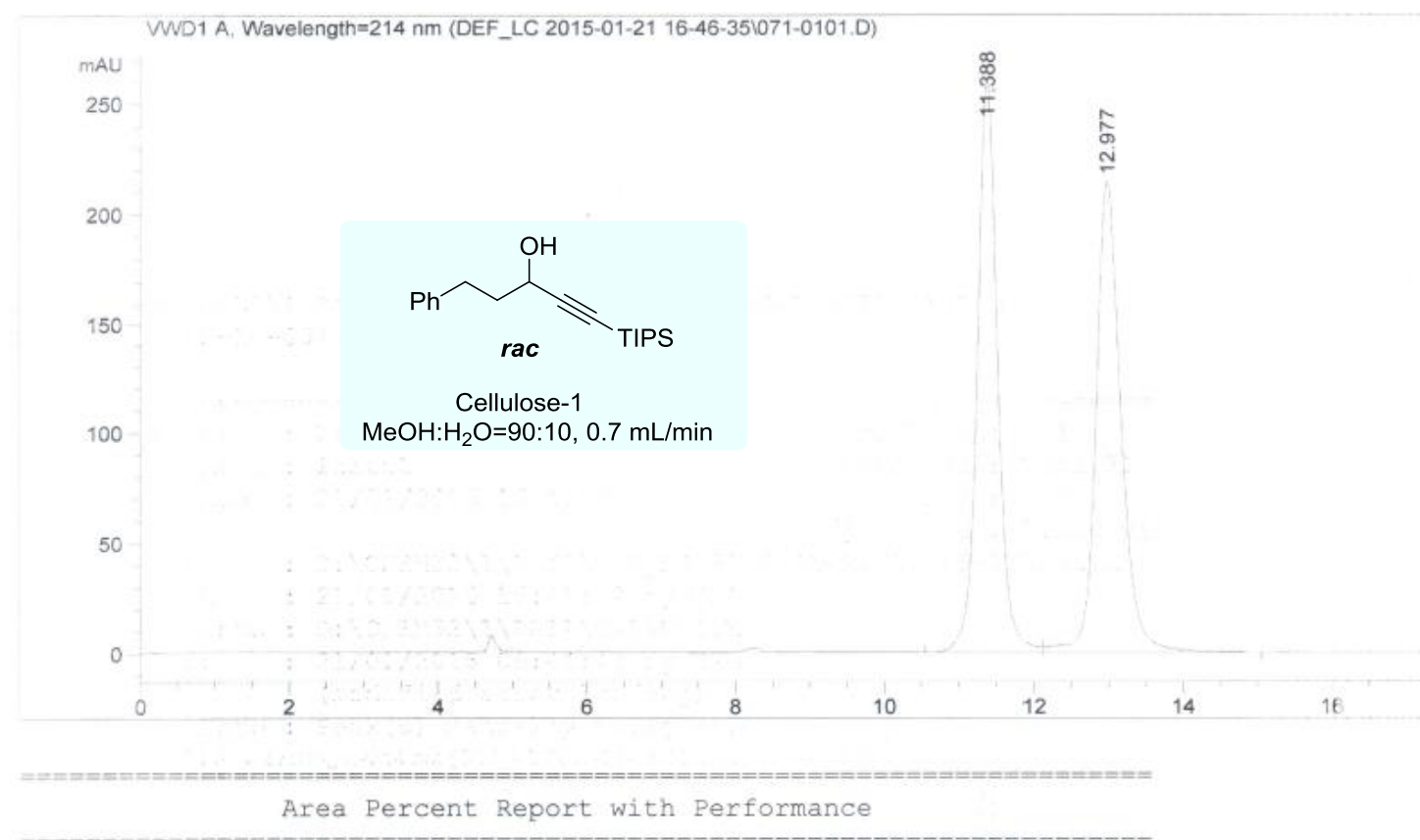

Signal 1: VWDI A, Wavelength=214 nm

\begin{tabular}{|c|c|c|c|c|c|c|c|c|}
\hline $\begin{array}{c}\text { RetTime } \\
\text { [min] }\end{array}$ & $k^{\prime}$ & $\begin{array}{c}\text { Area } \\
{\left[\mathrm{mAU}{ }^{*} \mathrm{~s}\right]}\end{array}$ & $\begin{array}{l}\text { Height } \\
\text { [mAU] }\end{array}$ & Symm. & $\begin{array}{l}\text { Width } \\
\text { [min] }\end{array}$ & Plates & $\begin{array}{l}\text { Resol } \\
\text { ution }\end{array}$ & $\begin{array}{l}\text { Select } \\
\text { ivity }\end{array}$ \\
\hline------ & -- & $\mid--\cdots-\cdots-\cdots$ & $----\infty-\infty$ & |---- & - - - - & $------\infty$ & $|-----|$ & |----- \\
\hline 11.388 & - & 4927.50098 & 260.06110 & 0.92 & 0.2774 & 9342 & - & - \\
\hline 12.977 & - & 4998.58105 & 214.29591 & 0.77 & 0.3381 & 8161 & 3.03 & 1.14 \\
\hline
\end{tabular}

$\star \star \star$ End of Report $\star \star \star ~$

WWD1 A, Wavelength $=214 \mathrm{~nm}(\mathrm{~W} \times L$ L20150120000023.D)

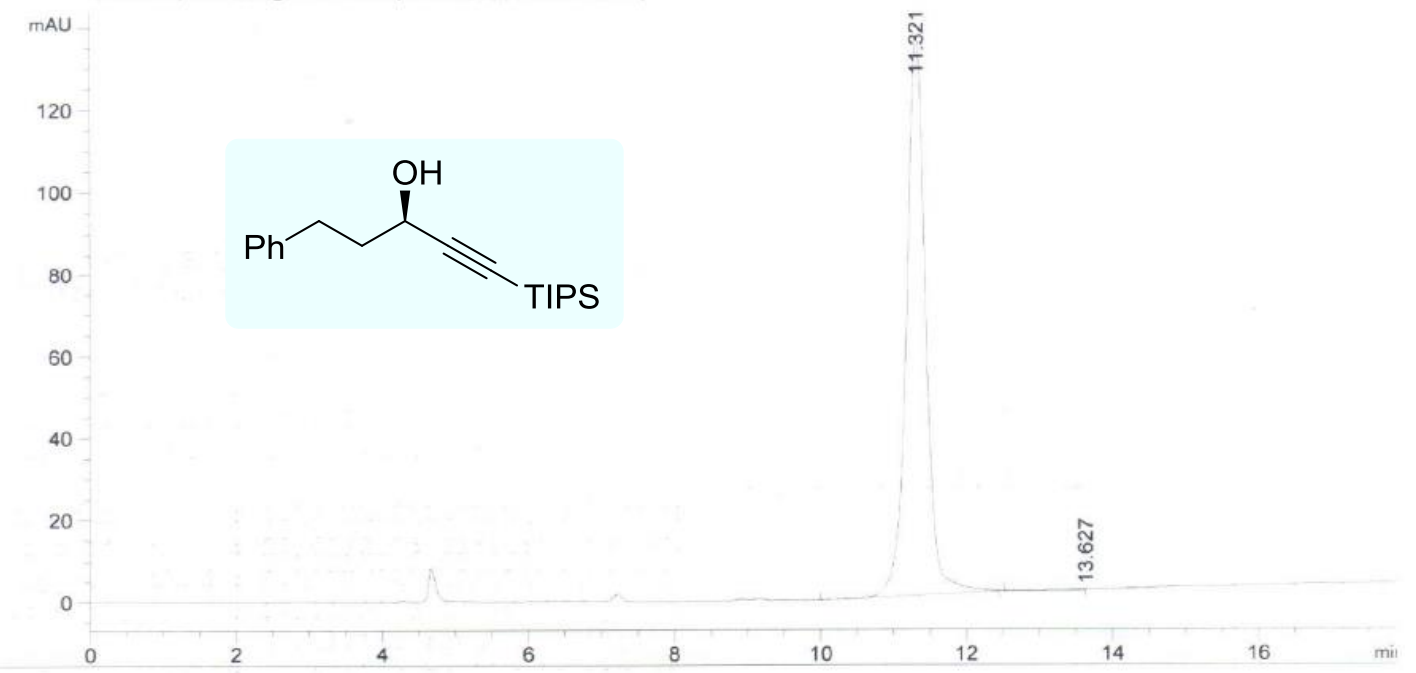


SignaI 1: VWD1 A, Wavelength=214 nm

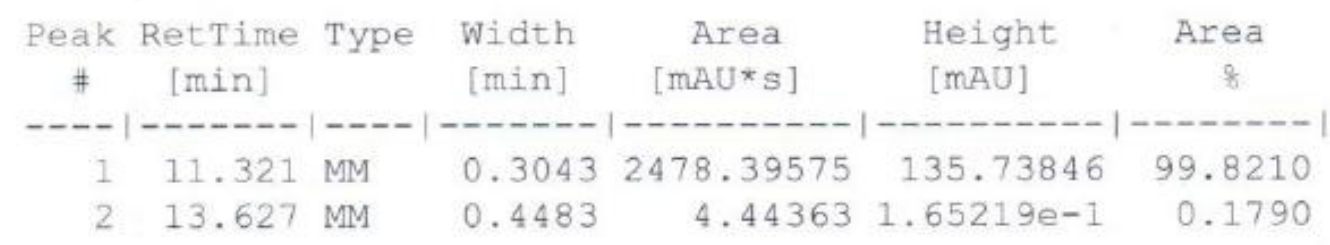

Totals :

$2482.83939 \quad 135.90368$

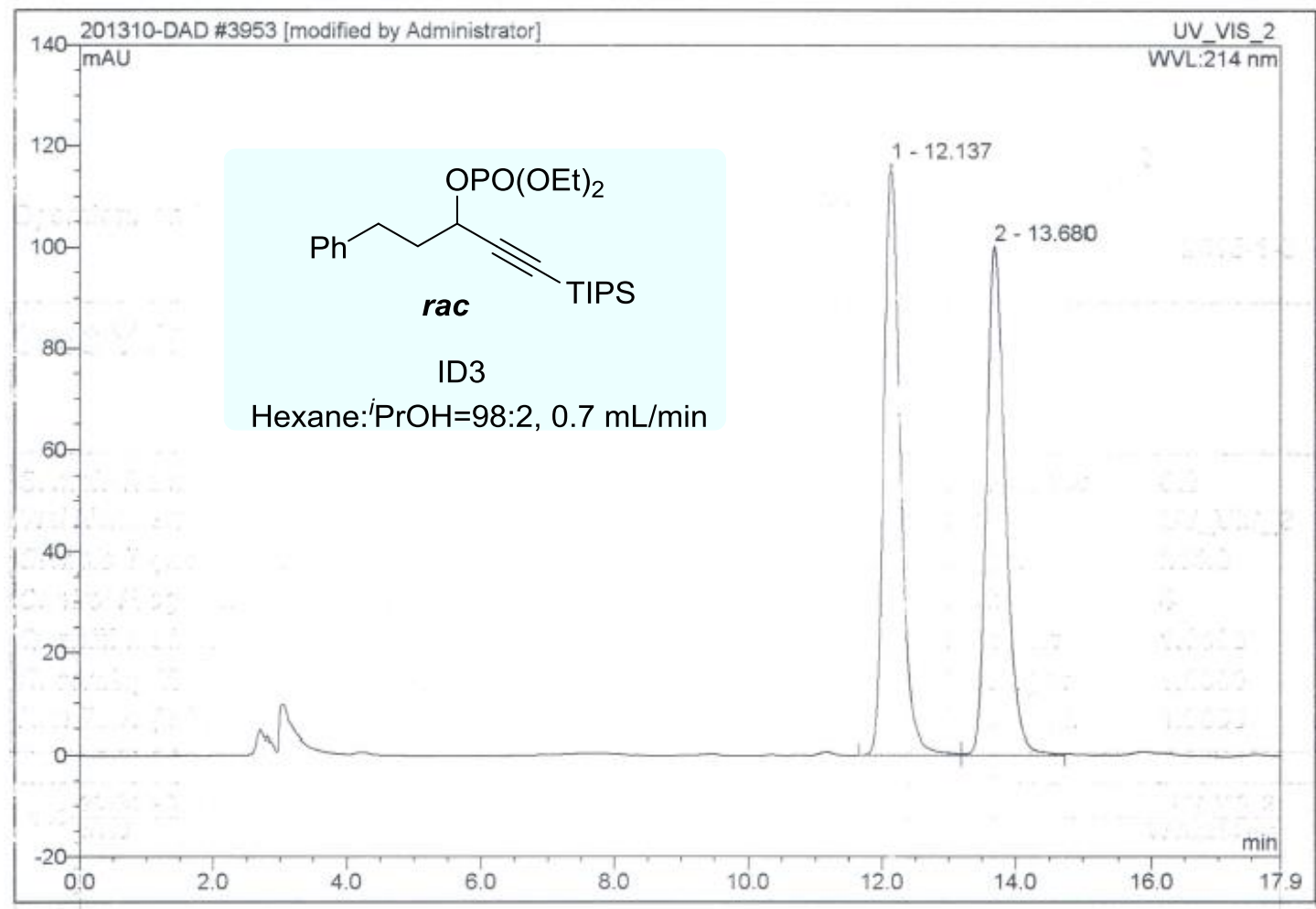

\begin{tabular}{|c|ccccccc|}
\hline No. & $\begin{array}{c}\text { Ret.Time } \\
\text { min }\end{array}$ & Peak Name & $\begin{array}{c}\text { Height } \\
\text { mAU }\end{array}$ & $\begin{array}{c}\text { Area } \\
\text { mAU*min }\end{array}$ & $\begin{array}{c}\text { Rel.Area } \\
\%\end{array}$ & Amount & Type \\
\hline 1 & 12.14 & n.a. & 116.376 & 33.618 & 50.35 & n.a. & BM $^{*}$ \\
2 & 13.68 & n.a. & 100.220 & 33.148 & 49.65 & n.a. & M * $^{*}$ \\
\hline Total: & & & 216.596 & 66.765 & 100.00 & 0.000 & \\
\hline
\end{tabular}




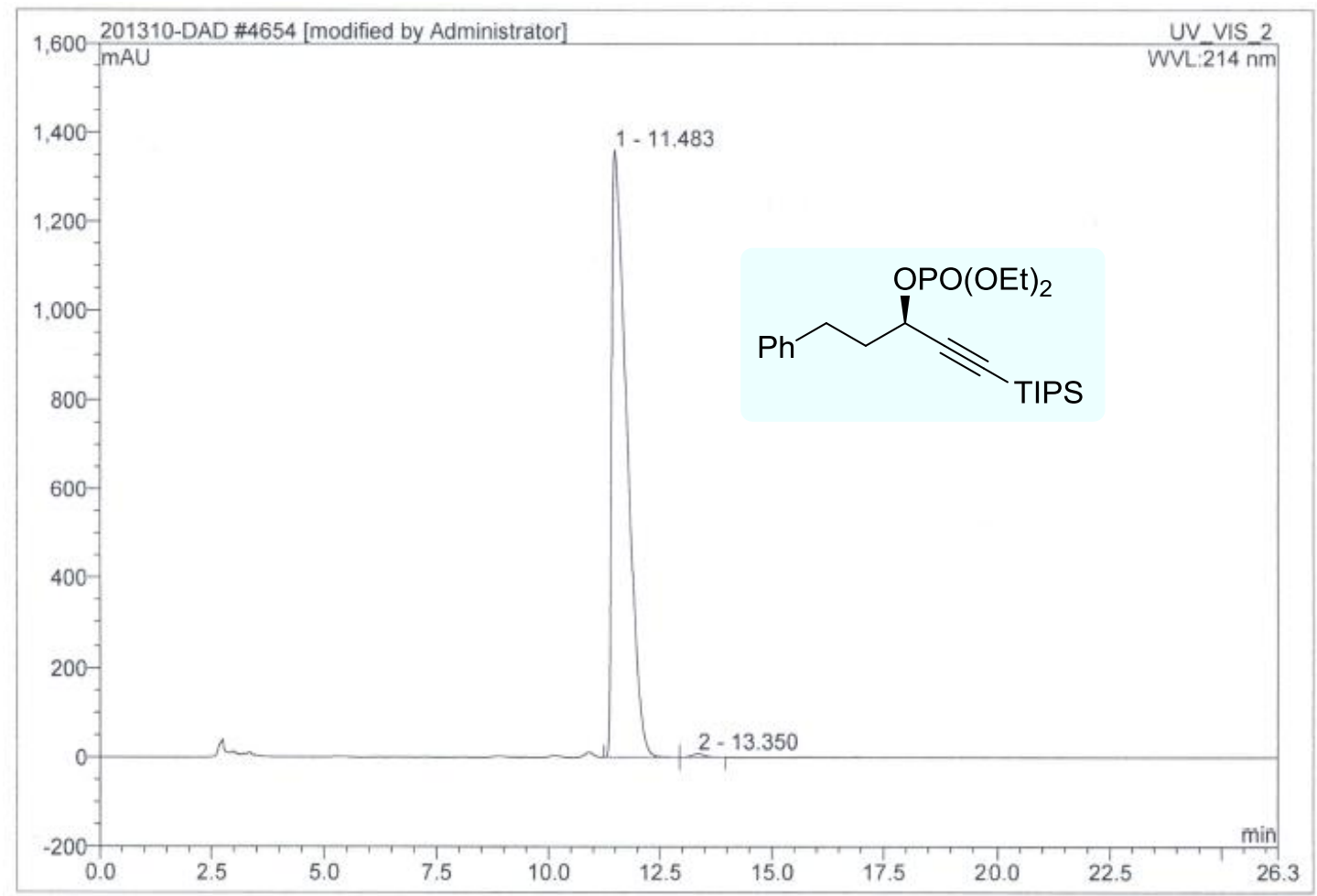

\begin{tabular}{|r|rrrrrrr|}
\hline No. & $\begin{array}{c}\text { Ret.Time } \\
\text { min }\end{array}$ & Peak Name & $\begin{array}{c}\text { Height } \\
\text { mAU }\end{array}$ & $\begin{array}{c}\text { Area } \\
\text { mAU*min }\end{array}$ & $\begin{array}{r}\text { Rel.Area } \\
\%\end{array}$ & Amount & Type \\
\hline 1 & 11.48 & n.a. & 1360.363 & 534.667 & 99.45 & n.a. & BM $^{*}$ \\
2 & 13.35 & n.a. & 8.401 & 2.930 & 0.55 & n.a. & MB $^{*}$ \\
\hline Total: & & & 1368.763 & 537.597 & 100.00 & 0.000 & \\
\hline
\end{tabular}

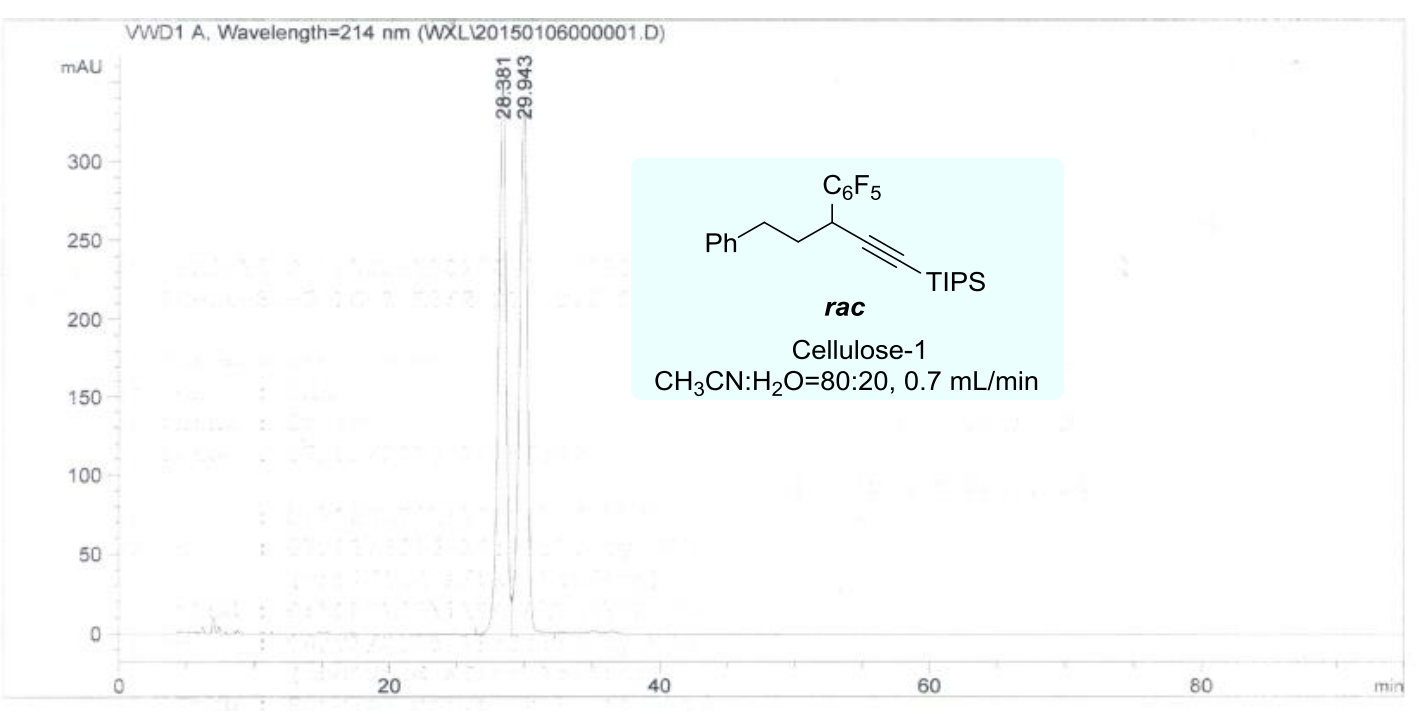

Area Percent Report 
Signal 1: VWD1 A, Wavelength=214 $\mathrm{nm}$

\begin{tabular}{|c|c|c|c|c|c|c|}
\hline $\begin{array}{c}\text { Proak } \\
\text { \# }\end{array}$ & $\begin{array}{c}\text { RatTimo } \\
\text { [min] }\end{array}$ & TyPe & $\begin{array}{l}\text { Width } \\
\text { [min] }\end{array}$ & $\begin{array}{c}\mathrm{Ax} \theta a \\
{\left[\mathrm{mAU}{ }^{*} \mathrm{~s}\right]}\end{array}$ & $\begin{array}{l}\text { Height } \\
\text { [mAU] }\end{array}$ & $\begin{array}{c}\text { Ara } \\
\frac{8}{6}\end{array}$ \\
\hline & & & & & & \\
\hline 1 & 28.381 & $M F$ & 0.6637 & 1. $39522 \mathrm{e} 4$ & 350.37167 & 50.0763 \\
\hline 2 & 29.943 & EM & 0.6898 & 1. $39097 \mathrm{e} 4$ & 336.06897 & 49.9237 \\
\hline
\end{tabular}

Totals : $\quad 2.78619 \mathrm{e} 4 \quad 686.44064$

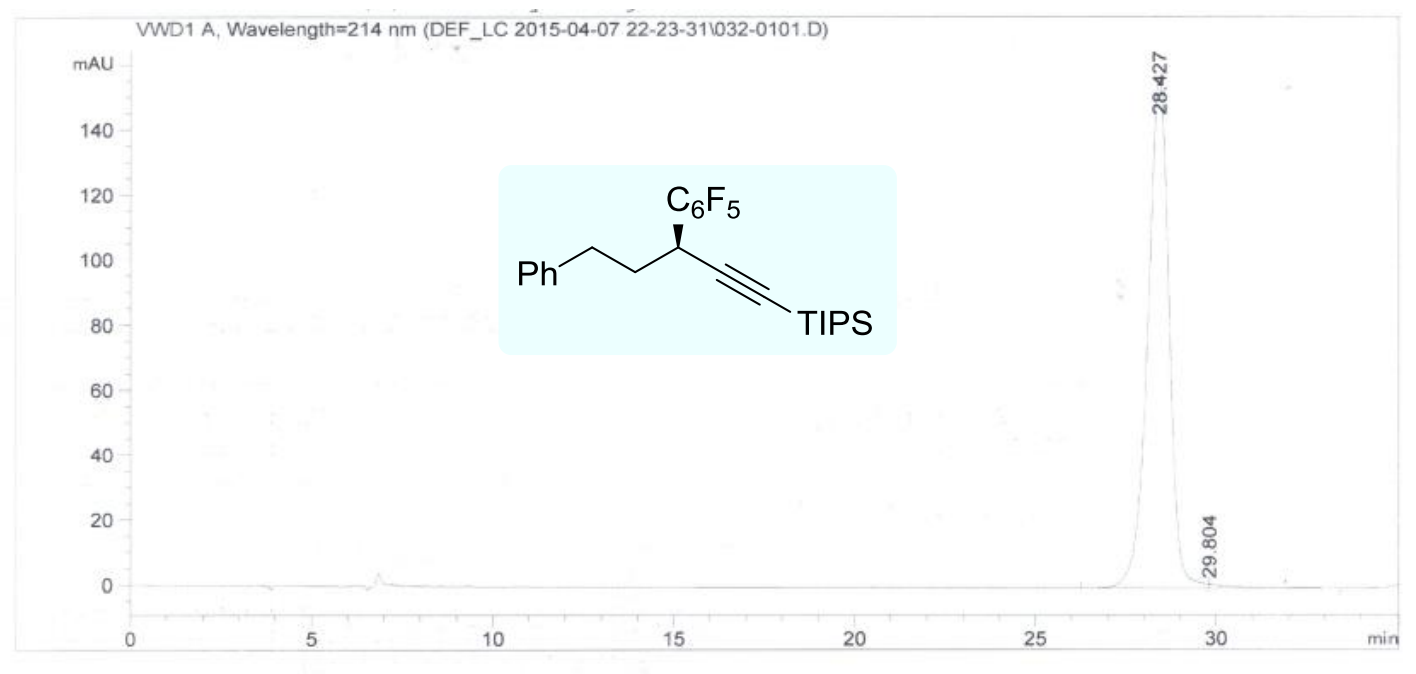

Area Percent Report

Signal 1: VWD1 A, Wavelength=214 nm

\begin{tabular}{|c|c|c|c|c|c|c|}
\hline $\begin{array}{c}\text { Peak } \\
\#\end{array}$ & $\begin{array}{l}\text { RetTime } \\
\text { [min] }\end{array}$ & Type & $\begin{array}{l}\text { Width } \\
\text { [min] }\end{array}$ & $\begin{array}{c}\text { Area } \\
{\left[\mathrm{mAU}^{\star} \mathrm{S}\right]}\end{array}$ & $\begin{array}{l}\text { Height } \\
\text { [mAU] }\end{array}$ & $\begin{array}{c}\text { Area } \\
8\end{array}$ \\
\hline 1 & 28.427 & $\mathrm{MF}$ & 0.6870 & 6488,23975 & 157.40114 & 99.1138 \\
\hline 2 & 29.804 & FM & 0.8018 & 58.01460 & 1.20593 & 0.8862 \\
\hline ota. & : & & & 6546.25434 & 158.60707 & \\
\hline
\end{tabular}

